Old Dominion University

ODU Digital Commons

Mechanical \& Aerospace Engineering Theses \&

Dissertations

Mechanical \& Aerospace Engineering

Spring 2016

\title{
Secondary Electron Emission from Plasma Processed Accelerating Cavity Grade Niobium
}

\author{
Miloš Bašović \\ Old Dominion University, milos8basovic@gmail.com
}

Follow this and additional works at: https://digitalcommons.odu.edu/mae_etds

Part of the Materials Science and Engineering Commons, Mechanical Engineering Commons, and the Physics Commons

\section{Recommended Citation}

Bašović, Miloš. "Secondary Electron Emission from Plasma Processed Accelerating Cavity Grade Niobium" (2016). Doctor of Philosophy (PhD), Dissertation, Mechanical \& Aerospace Engineering, Old Dominion University, DOI: $10.25777 / 7 z x v-7 h 28$

https://digitalcommons.odu.edu/mae_etds/2

This Dissertation is brought to you for free and open access by the Mechanical \& Aerospace Engineering at ODU Digital Commons. It has been accepted for inclusion in Mechanical \& Aerospace Engineering Theses \& Dissertations by an authorized administrator of ODU Digital Commons. For more information, please contact digitalcommons@odu.edu. 


\title{
SECONDARY ELECTRON EMISSION FROM PLASMA PROCESSED
}

\section{ACCELERATING CAVITY GRADE NIOBIUM}

\author{
by \\ Miloš Bašović \\ B.Sc. September 2008, University of Belgrade, Serbia \\ M.Sc. July 2010, University of Belgrade, Serbia \\ A Dissertation Submitted to the Faculty of \\ Old Dominion University in Partial Fulfillment of the \\ Requirements for the Degree of \\ DOCTOR OF PHILOSOPHY \\ MECHANICAL ENGINEERING \\ OLD DOMINION UNIVERSITY \\ May 2016
}

Approved by:

Mileta M. Tomović (Co-Director)

Gene J.-W. Hou (Co-Director)

Han P. Bao (Member)

Miltiadis D. Kotinis (Member)

Leposava Vušković (Member) 


\author{
ABSTRACT \\ SECONDARY ELECTRON EMISSION FROM PLASMA PROCESSED \\ ACCELERATING CAVITY GRADE NIOBIUM \\ Miloš Bašović \\ Old Dominion University, 2016 \\ Co-Directors: Dr. Mileta M. Tomović \\ Dr. Gene J.-W. Hou
}

Advances in the particle accelerator technology have enabled numerous fundamental discoveries in 20th century physics. Extensive interdisciplinary research has always supported further development of accelerator technology in efforts of reaching each new energy frontier.

Accelerating cavities, which are used to transfer energy to accelerated charged particles, have been one of the main focuses of research and development in the particle accelerator field. Over the last fifty years, in the race to break energy barriers, there has been constant improvement of the maximum stable accelerating field achieved in accelerating cavities. Every increase in the maximum attainable accelerating fields allowed for higher energy upgrades of existing accelerators and more compact designs of new accelerators. Each new and improved technology was faced with ever emerging limiting factors.

With the standard high accelerating gradients of more than $25 \mathrm{MV} / \mathrm{m}$, free electrons inside the cavities get accelerated by the field, gaining enough energy to produce more electrons in their interactions with the walls of the cavity. The electron production is exponential and the electron energy transfer to the walls of a cavity can trigger detrimental processes, limiting the performance of the cavity. The root cause of the free electron number gain is a phenomenon called Secondary Electron Emission (SEE). Even though the phenomenon has been known and studied over a century, there are still no effective means of controlling it. The ratio between the 
electrons emitted from the surface and the impacting electrons is defined as the Secondary Electron Yield $(S E Y)$. A SEY ratio larger than 1 designates an increase in the total number of electrons. In the design of accelerator cavities, the goal is to reduce the $S E Y$ to be as low as possible using any form of surface manipulation.

In this dissertation, an experimental setup was developed and used to study the $S E Y$ of various sample surfaces that were treated by different techniques. Specifically, this work provides the results of $S E Y$ from the plasma cleaned cavity grade niobium $(\mathrm{Nb})$ samples. Pure niobium is currently the material of choice for the fabrication of Superconducting Radio Frequency $(S R F)$ cavities. The effect of plasma processing with two different gases will be examined in two groups of samples. The first group of samples is made from cavity grade niobium. The second group of samples is made from the same material, but include a welded joint made by electron beam welding, since in niobium $S R F$ cavities the peak electric and magnetic field are seen in close proximity to the welded joints. Both groups of samples will be exposed to nitrogen $\left(\mathrm{N}_{2}\right)$ and a mixture of argon with oxygen $(\mathrm{Ar} / \mathrm{O} 2)$ plasma. It is the goal of this research to determine the $S E Y$ on these two groups of samples before and after plasma processing as a function of the energy of primary electrons. The $S E Y$ as a function of the angle of incidence of the primary electrons is tested on the samples treated with $\mathrm{Ar} / \mathrm{O}_{2}$ plasma. 
Copyright, 2016, by Miloš Bašović, All Rights Reserved. 
To my parents, Vukoman and Ubavka 


\section{ACKNOWLEDGMENTS}

I would like to begin by expressing gratitude to my advisor, Dr. Mileta M. Tomović, for the opportunity to join Old Dominion University as his graduate student and allowed me to take part in a multidisciplinary team of scientists and engineers. His guidance and mentorship throughout my doctoral studies was invaluable by providing me with all necessary tools to grow from a dedicated student to a critical thinker. My heartfelt thanks go to Dr. Leposava Vušković and Dr. Svetozar Popović for assigning me to tackle one of the problems from their research group. They have accepted me into their research team of graduate students and opened the doors of their laboratory, and supported this research with all necessary resources, knowledge and guidance needed to complete the experiments presented in this dissertation. Under their mentorship, I have gained considerable experience as an experimentalist and a researcher. I would like to thank Dr. Gene Hou for his academic guidance during my time spent as a graduate student. Advices and support provided by him have kept me optimistic through some of the most challenging periods of my research. Gratitude is also due to Dr. Miltiadis Kotinis and Dr. Han Bao for serving as members of my dissertation committee and their role as professors during my academic studies.

I sincerely appreciate the challenge to serve as a member on this multidisciplinary research

project. Their mentoring over the years have honed my scientific and experimental skills and helped me obtain knowledge related to various facets of engineering and science, both in understanding of complicated theoretical concepts, and the application challenges through indepth experimentation. The work presented in this dissertation would not have been possible without their advising and limitless patience. I feel very fortunate to have been mentored by 
these experts in their respective fields. The influence of the time spent with them at Old Dominion University, on my future life, cannot be overstated.

I also wish to show my thanks by mentioning the important role of the faculty and staff of the Departments of Mechanical and Aerospace Engineering, Physics, and Engineering Technology for their support and professionalism during my research. I would like to acknowledge financial support received from Thomas Jefferson National Accelerator Facility through Graduate Student Research Assistantship. My deepest thanks to the Department of Engineering Technology for an opportunity to work as an Adjunct Professor during my final year at Old Dominion University. I would like to recognize the contribution from Olga Trofimova, affiliated with the College of William and Mary and the Applied Research Center, Surface Characterization Lab for her work in performing the surface analysis of the samples.

I want to thank for all encouragement and support throughout this entire experience given to me by my family and friends in Serbia. Special thanks go to my friends Milka, Mladen, Ivan, Ana, Filip, Janardan, Marija, Vukica, Radovan, and Sasa, for making Old Dominion University a great place to work at, and Norfolk a home away from home.

${ }^{1}$ Jefferson Laboratory is operated by Jefferson Science Associates under DOE Contract No. DE-AC05-06OR23177. 


\section{NOMENCLATURE}

SEE Secondary electron emission

SEY Secondary electron yield

SRF Superconducting radio frequency

$T_{c} \quad$ Critical temperature for superconductivity

$R R R \quad$ Residual resistivity ratio

$E_{\text {acc }} \quad$ Accelerating gradient

$V_{a c c} \quad$ Accelerating voltage

dength of the cavity

$Q_{0} \quad$ Intrinsic quality factor

$\omega \quad$ Angular resonant frequency

U Energy stored by electromagnetic field

$P_{c} \quad$ Dissipated power

rf radiofrequency

$B C P \quad$ Buffered chemical polishing

ECP Electro-chemical polishing

HPR High pressure rinsing

SEM Scanning electron microscope

$\delta \quad$ SEY

$\delta_{\max } \quad$ Maximum SEY

$E_{0} \quad$ Primary electron energy

$E_{0 I} \quad$ Primary electron energy of the first crossover point

$E_{\text {OII }} \quad$ Primary electron energy of the second crossover point 
$E_{0 \max } \quad$ Primary electron energy at maximum SEY

EDC Energy distribution curve

$\theta \quad$ Incident angle of the primary electrons

WZ Weld zone

HAZ Heat affected zone

BASE Base metal surface, sample set

R-HAZ Heat treated base metal surface

WELD Sample set

OFFSET Sample set

$i_{p} \quad$ Primary electron beam current

$i_{s} \quad$ Sample current

$i_{c} \quad$ Collector current

XPS X-ray photoelectron spectroscopy

$R G A \quad$ Residual gas analyzer

SIMS Secondary ion mass spectroscopy

$(x),(y),(z) \quad$ Translational axes of motion

$R(y) \quad$ Rotational motion around (y) axis

GUI Graphical user interface

$U_{c} \quad$ Collector bias voltage

$U_{s} \quad$ Sample bias voltage

PBC Primary beam collector

$i_{B P} \quad$ Current of the back plate

$i_{F P} \quad$ Current of the front plate 
$A \quad$ Ratio of the $i_{B P}$, and sum of the $i_{B P}$ and $i_{F P}$

$\bar{\delta} \quad$ Average SEY

$s$

Sample standard deviation

$\eta$

Material characteristic parameter of angular dependence of SEY

$B P P \quad$ Before plasma processing

$A P P \quad$ After plasma processing

AFM Atomic force microscope

EDS Energy-dispersive x-ray spectroscopy 


\section{TABLE OF CONTENTS}

Page



LIST OF FIGURES …..................................................................................................

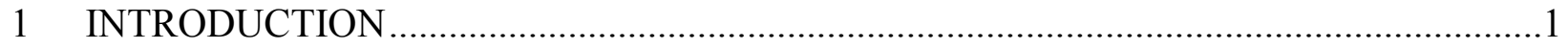

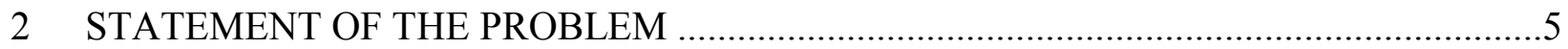

2.1 Performance of accelerating cavities ................................................................ 7

2.2 Secondary electron emission..................................................................... 12

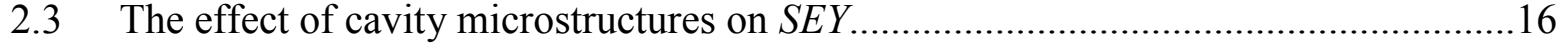

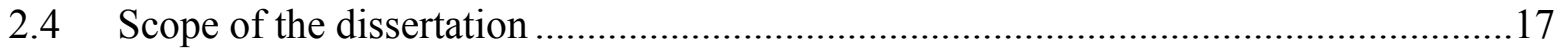

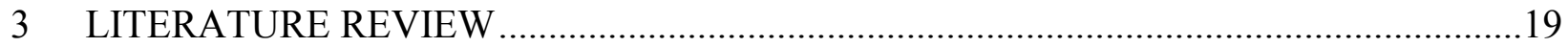

3.1 SEY measurement techniques ........................................................................ 19

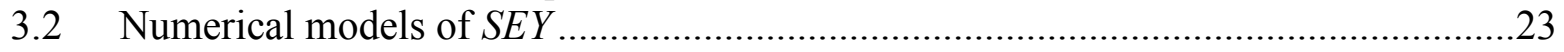

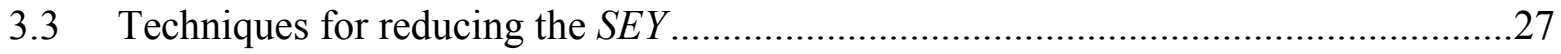

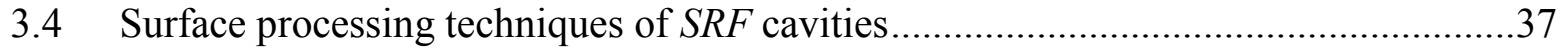

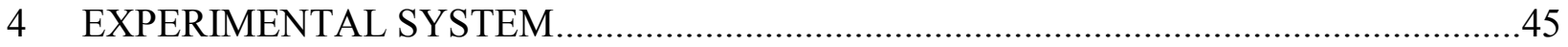

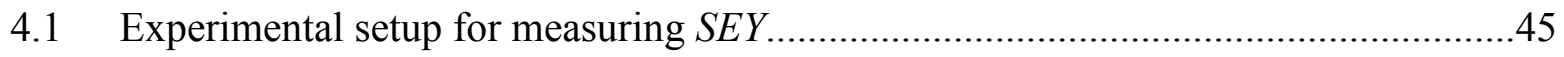

4.2 Calibration measurements.............................................................................67

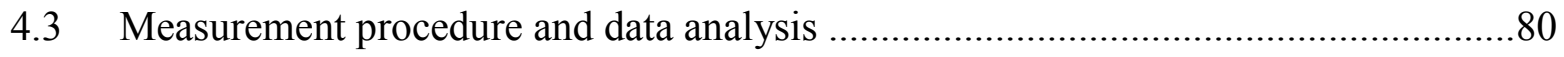

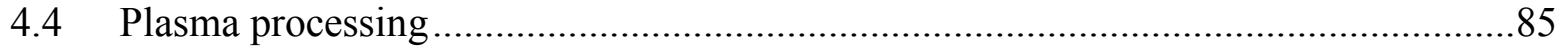

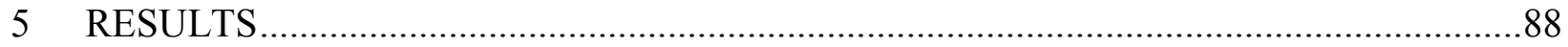

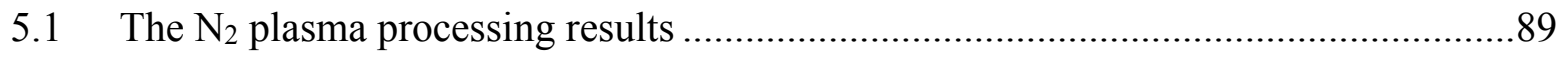

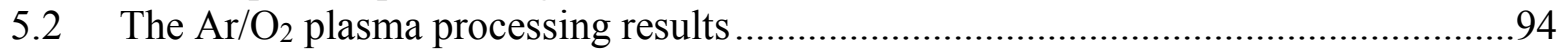

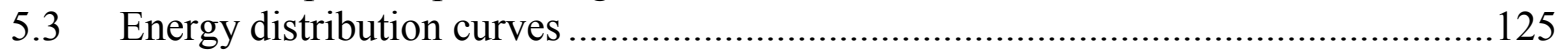

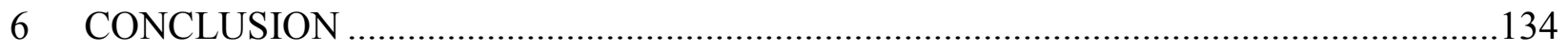

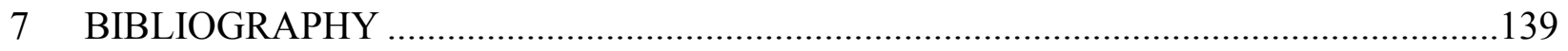

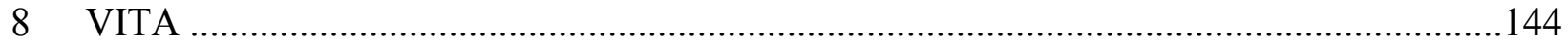




\section{LIST OF TABLES}

Table

Page

4.1 List of impurities in niobium sheet metal, sorted by the total content (maximum percent). Chemical content was determined by the Eagle Alloys Corporation.

4.2 Absolute sample stage coordinates for the middle row/center column spot on the surface at all incident angles. Distance from the electron gun mouth was denoted by (z) axis, vertical position was denoted by (y) axis, and horizontal position was denoted by (x) axis....... 62

4.3 The electron beam parameters used for the $S E Y$ measurements. 78

5.1 Average maximum yield ( $\bar{\delta}_{\max }$ ) and corresponding energy $\left(E_{0}\right)$, (a) before and (b) after plasma processing at $\theta=0^{\circ}$.

5.2 Average maximum yield ( $\bar{\delta}_{\max }$ ) and corresponding energy $\left(E_{0}\right)$, (a) before and (b) after plasma processing at $\theta=+15^{\circ}$.

5.3 Average maximum yield $\left(\bar{\delta}_{\max }\right)$ and corresponding energy $\left(E_{0}\right)$, (a) before and (b) after plasma processing at $\theta=-15^{\circ}$.

5.4 Average maximum yield $\left(\bar{\delta}_{\max }\right)$ and corresponding energy $\left(E_{0}\right)$, (a) before and (b) after plasma processing at $\theta=+30^{\circ}$.

5.5 Average maximum yield $\left(\bar{\delta}_{\max }\right)$ and corresponding energy $\left(E_{0}\right)$, (a) before and (b) after plasma processing at $\theta=-30^{\circ}$.

5.6 Average maximum yield $\left(\bar{\delta}_{\max }\right)$ and corresponding energy $\left(E_{0}\right)$, (a) before and (b) after plasma processing at $\theta=+45^{\circ}$.

5.7 Average maximum yield $\left(\bar{\delta}_{\max }\right)$ and corresponding energy $\left(E_{0}\right)$, (a) before and (b) after plasma processing at $\theta=-45^{\circ}$.

5.8 Average maximum yield $\left(\bar{\delta}_{\max }\right)$ and corresponding energy $\left(E_{0}\right)$, (a) before and (b) after plasma processing at $\theta=+60^{\circ}$.

5.9 Average maximum yield $\left(\bar{\delta}_{\max }\right)$ and corresponding energy $\left(E_{0}\right)$, (a) before and (b) after plasma processing at $\theta=-60^{\circ}$. 
5.10 Values of parameter $\eta$ for characteristic surfaces, (a) before and (b) after plasma processing.

5.11 Surface statistics measured on a 50x50 $\mu \mathrm{m}$ surface area by $A F M$ on characteristic surfaces, (a) before and (b) after plasma processing... 


\section{LIST OF FIGURES}

Figure

Page

2.1 End section of a multi-cell cavity showing iris weld, equator weld, and stiffening ring locations [4]. 5

2.2 Typical $Q$ factor curve of a multi-cell niobium cavity [4] ........................................... 8

2.3 Temperature gradient of a cavity with a local temperature increase at 1, 2, 3, and 4 [10]... 11

2.4 Typical $S E Y(\delta)$ curve as a function of primary electron beam energy $\left(E_{0}\right)$ with

characteristic points. 14

2.5 Secondary electron energy distribution for stainless steel and $E_{0}=300 \mathrm{eV}$ at normal incident angle [14]. 15

2.6 $\operatorname{SEY}(\delta)$ as a function of $E_{0}$ and increase in incident angle $(\theta)$ measured on a molybdenum sample [16]. 16

2.7 Distribution of (a) electric and (b) magnetic field in a $S R F$ elliptical cavity...... 17

3.1 Schematic of the sample current method [19]. 20

3.2 Schematic of the collector current method [24].

3.3 $S E Y$ as a function of $E_{0}$, showing the agreement of different models represented by Eqs. (3.3-3.6) with the experimental results of niobium samples [3]. 25

3.4 Schematic representation of the commonly assumed model of the secondary electron formation depth as function of incident angle [32]. 26

3.5 $S E Y$ as a function of $E_{0}$, showing the effect of $100 \mathrm{~nm}$ carbon thin film deposited on stainless steel base [33].

3.6 $S E Y$ as a function of $E_{0}$, showing the effect of $20 \mathrm{~nm}$ carbon thin film deposited on the copper base [35].

3.7 SEY as a function of $E_{0}$, showing the effect of $14 \mathrm{~nm}$ of $\mathrm{NbN}$ and $\mathrm{NbC}$ deposited on (a) clean $\mathrm{Nb}$ sample. $S E Y$ increases (b) after air exposure, which was reduced with (c) the electron beam irradiation [36].

3.8 $S E Y$ as a function of $E_{0}$ of niobium thin layer deposited on copper base, showing the effect of heat treatment at (a) different temperatures and (b) different duration at $150{ }^{\circ} \mathrm{C}$ [17]. 
Figure

Page

3.9 $S E Y$ as a function of $E_{0}$, at different activation temperatures of (a) $\mathrm{TiZrV}$ and (b) $\mathrm{TiZr}$ thin films of $1 \mu \mathrm{m}$ thickness deposited on a copper base [38] ............................................... 33

3.10 SEY as a function of $E_{0}$, showing the effect of irradiation by an electron beam of 500 $\mathrm{eV}$ energy and $5 \mu \mathrm{A}$ of current on copper sample [34]....

3.11 SEY as a function of $E_{0}$, showing the effect of argon (Ar), nitrogen $\left(\mathrm{N}_{2}\right)$, methane $\left(\mathrm{CH}_{4}\right)$, and argon/oxygen $\left(\mathrm{Ar} / \mathrm{O}_{2}\right)$ glow discharge on niobium samples [3].

3.12 SEM micrograph of (a) base surface of the niobium sample [46], and (b) sample with the equator weld area [47], after $B C P$ etching.

3.13 SEM micrograph of (a) base surface of the niobium sample [46], and (b) sample with the equator weld area [47], after ECP etching. 40

4.1 Components of the $S E Y$ experimental setup. 46

4.2 A photograph of the experimental setup for measuring $S E Y$. 47

4.3 The $S E M$ pictures of three different microstructures of characteristic surfaces taken at x500 magnification scale. Surfaces correspond to the (a) WZ, (HAZ), and (c) BASE. 52

4.4 Sketch of the sample with a sectional view showing mounting hole. 54

4.5 Sketch of the samples from each set (top row) and their matching photographs (bottom row). Samples are from sets as follows: (a) BASE set, (b) OFFSET set, and (c) WELD set. 54

4.6 Sketch of the collector design (top) and cross-section of the collector with dimensions (bottom)......

4.7 3D model of the experimental setup interior showing the directions of translational ((x), $(\mathrm{y})$, and $(\mathrm{z})$ ) and rotational motions $\left(\mathrm{R}_{(\mathrm{y})}\right)$, and location of the electron gun with the mounted collector [54]. 58

4.8 Cross-section of the electron gun and the collector assembly, with a centered sample at normal incident angle.

4.9 Sketch of the samples from each set with the location of the 9 measurement spots, at normal incident angle.

4.10 Sketch of the incident angles $(\theta)$ that were formed by the normal of the sample surface and the primary electron beam. 
Figure

Page

4.11 Surface of the sample observed from the primary electron beam direction at different angle of sample inclinations and the number of measuring spots with locations for each angle.

4.12 The interface mechanism used to transfer the rotational motion of the stepper motors to micrometer calipers of translational motion, while allowing free axial movement.

4.13 (a) Assembled automated system for sample positioning, and (b) stepper motors removed from the sample stage.

4.14 Schematic of the experimental setup. The collector and the sample currents were measured simultaneously and the data were stored in $P C$.

4.15 Electron gun filament current at a constant filament voltage of $1.1 \mathrm{~V}$.

4.16 $S E Y$ as a function of primary electron energy $\left(E_{0}\right)$ for five different electron gun filament currents, with bias $\left(U_{c}=+40 \mathrm{~V}\right.$ and $\left.U_{s}=-20 \mathrm{~V}\right)$ and without bias $\left(U_{c}=0 \mathrm{~V}\right.$ and $U_{s}=$ $0 \mathrm{~V})$.

4.17 Primary electron beam current $\left(i_{0}\right)$ as a function of primary electron energy $\left(E_{0}\right)$ for five different electron gun filament currents, with bias $\left(U_{c}=+40 \mathrm{~V}\right.$ and $\left.U_{s}=-20 \mathrm{~V}\right)$ and without bias $\left(U_{c}=0 \mathrm{~V}\right.$ and $\left.U_{s}=0 \mathrm{~V}\right)$.

4.18 SEY as a function of the collector $\left(U_{c}\right)$ and the sample $\left(U_{s}\right)$ voltages for four primary electron beam energies $\left(E_{0}\right)$. 73

4.19 SEY as a function of the sample bias voltage $\left(U_{s}\right)$ at two collector potentials $\left(U_{c}\right)$ for four primary electron beam energies $\left(E_{0}\right)$.

4.20 SEY and primary electron beam current $\left(i_{p}\right)$ as a function of $E_{0}$, for (a) $U_{c}=+20 \mathrm{~V}$ and $U_{s}=-10 \mathrm{~V}$, and (b) $U_{c}=+30 \mathrm{~V}$ and $U_{s}=-10 \mathrm{~V}$.

4.21 Design sketch of the Primary Beam Collector $(P B C)$.

4.22 The ratio of electron currents $(A)$ for two diameters of the front plate hole $\left(d_{F P}\right)$ and Focus voltage, as a function of the primary electron energy $\left(E_{0}\right)$.

4.23 Three $S E Y$ curves recorded during an energy scan of a single measurement spot as a function of the primary electron beam energy. 83

5.1 Sketch of (a) BASE 1 and BASE 2 samples, and (b) $W Z$ sample used for $\mathrm{N}_{2}$ plasma processing, with the location of the electron beam during $S E Y$ measurement. 
5.2 The average primary electron beam current $\left(i_{0}\right)$ as a function of primary electron beam energy $\left(E_{0}\right)$, (a) before plasma processing [23], and (b) after plasma processing in nitrogen for $B A S E$ 1, BASE 2, and $W Z$ sample. Statistical bars are covering the standard deviation.

5.3 Average $S E Y$ as a function of $E_{0}$, for $B A S E 1, B A S E$ 2, and $W Z$ samples (a) before plasma processing [23], and (b) after plasma processing in nitrogen. Statistical bars are representing $90 \%$ prediction interval.

5.4 SEY curves (triangle markers) and corresponding $i_{0}$ (square markers) for the first of seven measurements before $(B P P)$ and after $(A P P)$ nitrogen plasma processing as a function of $E_{0}$, for (a) $B A S E 1$ and (b) $B A S E 2$ samples.

5.5 SEY curves (triangle markers) and corresponding $i_{0}$ (square markers) for the first of seven measurements before $(B P P)$ and after $(A P P)$ nitrogen plasma processing as a function of $E_{0}$, for WZ sample.

5.6 Average primary electron beam current $\left(i_{0}\right)$ and standard deviation as a function of $E_{0} \ldots . . .95$

5.7 The average $S E Y$ with sample standard deviation bars as a function of $E_{0}$ at $\theta=0^{\circ}$, (a) before and (b) after plasma processing.

5.8 The average $S E Y$ with $90 \%$ prediction interval bars as a function of $E_{0}$ at $\theta=0^{\circ}$, (a) before and (b) after plasma processing. 98

5.9 The average $S E Y$ with sample standard deviation bars as a function of $E_{0}$ at $\theta=+15^{\circ}$, (a) before and (b) after plasma processing. 100

5.10 The average $S E Y$ with $90 \%$ prediction interval bars as a function of $E_{0}$ at $\theta=+15^{\circ}$, (a) before and (b) after plasma processing. 101

5.11 The average $S E Y$ with sample standard deviation bars as a function of $E_{0}$ at $\theta=-15^{\circ}$, (a) before and (b) after plasma processing. 102

5.12 The average $S E Y$ with $90 \%$ prediction interval bars as a function of $E_{0}$ at $\theta=-15^{\circ}$, (a) before and (b) after plasma processing. 104

5.13 The average $S E Y$ with sample standard deviation bars as a function of $E_{0}$ at $\theta=+30^{\circ}$, (a) before and (b) after plasma processing. 105

5.14 The average $S E Y$ with $90 \%$ prediction interval bars as a function of $E_{0}$ at $\theta=+30^{\circ}$, (a) before and (b) after plasma processing. 106

5.15 The average $S E Y$ with sample standard deviation bars as a function of $E_{0}$ at $\theta=-30^{\circ}$, (a) before and (b) after plasma processing. 
5.16 The average $S E Y$ with $90 \%$ prediction interval bars as a function of $E_{0}$ at $\theta=-30^{\circ}$, (a) before and (b) after plasma processing. 108

5.17 The average $S E Y$ with sample standard deviation bars as a function of $E_{0}$ at $\theta=+45^{\circ}$, (a) before and (b) after plasma processing.

5.18 The average $S E Y$ with $90 \%$ prediction interval bars as a function of $E_{0}$ at $\theta=+45^{\circ}$, (a) before and (b) after plasma processing.

5.19 The average $S E Y$ with sample standard deviation bars as a function of $E_{0}$ at $\theta=-45^{\circ}$, (a) before and (b) after plasma processing.

5.20 The average $S E Y$ with $90 \%$ prediction interval bars as a function of $E_{0}$ at $\theta=-45^{\circ}$, (a) before and (b) after plasma processing.

5.21 The average $S E Y$ with sample standard deviation bars as a function of $E_{0}$ at $\theta=+60^{\circ}$, (a) before and (b) after plasma processing.

5.22 The average $S E Y$ with $90 \%$ prediction interval bars as a function of $E_{0}$ at $\theta=+60^{\circ}$, (a) before and (b) after plasma processing.

5.23 The average $S E Y$ with sample standard deviation bars as a function of $E_{0}$ at $\theta=-60^{\circ}$, (a) before and (b) after plasma processing. 116

5.24 The average $S E Y$ with $90 \%$ prediction interval bars as a function of $E_{0}$ at $\theta=-60^{\circ}$, (a) before and (b) after plasma processing.

5.25 SEY as a function of $\theta$ for $E_{0}=320 \mathrm{eV}$ of $B A S E$ surface, (a) before and (b) after plasma processing. Statistical bars represent the range of $90 \%$ prediction interval.

5.26 SEY as a function of $\theta$ for $E_{0}=320 \mathrm{eV}$ of $W Z$ surface, (a) before and (b) after plasma processing. Statistical bars represent the range of $90 \%$ prediction interval.

5.27 SEY as a function of $\theta$ for $E_{0}=320 \mathrm{eV}$ of $H A Z$ surface, (a) before and (b) after plasma processing. Statistical bars represent the range of $90 \%$ prediction interval.

5.28 SEY as a function of $\theta$ for $E_{0}=320 \mathrm{eV}$ of $R-H A Z$ surface, (a) before and (b) after plasma processing. Statistical bars represent the range of $90 \%$ prediction interval.

5.29 SEM micrographs of BASE surface at x1,000 magnification, (a) before and (b) after plasma processing. Granular inclusions on the surface (a) were almost completely removed by processing in $\mathrm{Ar} / \mathrm{O}_{2}$ plasma for 1 hour. 
5.30 SEM micrographs of the BASE surface at x50,000 magnification, (a) before and (b) after plasma processing.

5.31 EDS spectra measured at the high concentration of granular inclusions on $B A S E$ surface, (a) before and (b) after plasma processing. A relative increase in the oxygen content can be observed after plasma processing.

5.32 EDS spectra measured on a characteristic BASE surface, (a) before and (b) after plasma processing. A spectral line after plasma processing indicates an increase in oxygen content on the BASE surface.

5.33 Current measured on the collector $\left(i_{c}\right)$ and the $B A S E$ sample $\left(i_{s}\right)$ for indicated $E_{0}$, as a function of the collector retarding potential.

5.34 Primary electron beam current $\left(i_{0}\right)$ calculated as the sum of collector and BASE sample currents for indicated $E_{0}$, as a function of the collector retarding potential.

5.35 (a) Normalized collector current as a function of retarding potential and (b) corresponding $E D C$, at $E_{0}=140 \mathrm{eV}$.

5.36 Normalized collector current as a function of the retarding potential for copper sample $[56]$.

$5.37 E D C$ of $B A S E$ sample measured before $(B P P)$ and after $(A P P)$ plasma processing as a function of secondary electron energy at $E_{0}=140 \mathrm{eV}$.

$5.38 E D C$ of $B A S E$ sample measured before $(B P P)$ and after $(A P P)$ plasma processing as a function of secondary electron energy at $E_{0}=180 \mathrm{eV}$.

$5.39 E D C$ of $B A S E$ sample measured before $(B P P)$ and after $(A P P)$ plasma processing as a function of secondary electron energy at $E_{0}=300 \mathrm{eV}$. 


\section{CHAPTER 1}

\section{INTRODUCTION}

Modern accelerator physics started at the beginning of the 1930s with the CockcroftWalton generator [1] which allowed the first artificial change of the atomic nuclei. In an accelerator, charged particles attain near relativistic speeds through an applied electric field confined in accelerating cavities. At the time, the achievable energy of the particles was below 1 $\mathrm{MeV}$. Since then, the constant requirement for higher accelerating energies has driven the development of accelerators in energy scale and towards more efficient methods of achieving increased energy scale. Today, energy attainable in particle accelerators can reach several $\mathrm{TeV}$. As the energy in the accelerators increased, it allowed scientists to detect ever smaller scales of matter. It can be said that the development of twentieth century physics can be greatly attributed to the development of particle accelerators.

As the energy levels of accelerators grew so did the scale of the accelerators, from a machine that can be placed in a room to $27 \mathrm{~km}$ long storage rings at the European Organization for Nuclear Research $(C E R N)$. With every new generation of particle accelerators, technology requirements to build and operate one grew and created the need for a large number of complementary research areas. These research areas provided the means of further improving on the design of accelerators. A number of technologies originally developed for the need of particle accelerators found application outside of their original intent, such as the development of the Internet. 
Acceleration of particles in accelerators occurs in a specially designed resonator cavities. Particles accelerate by receiving the energy from the electromagnetic field confined inside the cavity. In order to reduce the energy losses and scattering of the particle bunches from collision with air or other particles present, the cavities are pumped down to a vacuum level of $10^{-11}$ Torr or better. In addition, cavities had gone through a significant redesign in order to achieve higher energy levels. As the central component of an accelerator, the cavity has been extensively studied for various limiting factors that occur with the increase in an applied electric field. Shape, field emission, multipacting, material, and others were and still are some of the limiting factors in achievable acceleration. Of particular interest is the formation and mitigation of electron cloud inside the cavities. Specifically, it has been determined that electron cloud forms and grows due to the existence of free charged particles (particles not being accelerated) and the Secondary Electron Emission ( $S E E)$. Definition of secondary electrons in this work encompasses all electrons emitted from the examined surface, regardless of their energy level or formation mechanism. Free particles, under the influence of the high electromagnetic field confined inside the cavity, are accelerated, and at certain conditions, can impact the surface releasing the additional particles. The ratio between the number of electrons leaving the surface and the impacting electrons is defined as Secondary Electron Yield $(S E Y)$. An increase in the $S E Y$ in a confined space can have a detrimental effect on the acceleration, limiting the effectiveness of the cavities.

Various surface treatments have proven to be successful in improving the performance of cavities, but still the theoretical limit is yet to be achieved. It would appear that current surface processing technologies are reaching their potential and in order to continue increasing the accelerating gradient of cavities, alternative methods of surface preparation need to be examined. 
Plasma processing was proven as an effective method for processing the surfaces in research areas of semiconductor and medical applications. Recently developed technology might lead to an efficient method of processing the accelerating cavities [2]. Although the effect of plasma on the various accelerator materials has been studied so far, further experimental research is needed to determine the $S E E$ from the cavity surface in order to provide comprehensive data for simulations of cavity performance.

The objective of this work was to study the effects of plasma processing on the $S E Y$ at the surface of niobium samples at a wide range of energies $(60-2000 \mathrm{eV})$ of impacting electrons. The effects of electrons impacting the surface were observed at various impacting angles in the range from $-60^{\circ}$ to $+60^{\circ}$ around the angle of normal incidence with the $15^{\circ}$ angle increment. An angle at which electrons impact the surface is a probabilistic event, and for that purpose, it is worthwhile to determine the effect of the incident angle of primary electrons on the $S E Y$ of niobium. In order to describe the effects of plasma on different cavity surfaces found within cavities, two groups of samples were used. All samples were made from cavity grade niobium, but one group included a welded joint across its surface since it was shown that inside the niobium $S R F$ cavity electromagnetic field magnitudes were significant in close proximity to the welded joints. Both groups of samples were exposed to nitrogen $\left(\mathrm{N}_{2}\right)$ and a mixture of argon with oxygen $\left(\mathrm{Ar} / \mathrm{O}_{2}\right)$ plasma operating at 50 mTorr pressure, since the reduction of $S E Y$ after plasma processing was reported [3].

This dissertation is organized as follows. Chapter 1 provides a brief introduction to the presented work, including the motivation for the research. Chapter 2 describes the research problem in detail and discusses possible solution approaches. Chapter 3 provides an overview of the relevant literature which includes the measurement techniques, numerical models, commonly 
used methods for reducing the $S E Y$, and available technologies for processing the $S R F$ accelerating cavities. Chapter 4 presents an experimental setup and measurement procedure used in the study. Chapter 5 gives the results of $S E Y$ measurements before and after plasma processing at several incident angles of primary electrons. Finally, Chapter 6 provides the conclusions and recommendations for future work. 


\section{CHAPTER 2}

\section{STATEMENT OF THE PROBLEM}

Cavities are a special type of resonator designed to confine the electromagnetic field and transfer the energy to the accelerating particles. With the development of accelerators, cavities have undergone significant shape alterations in order to achieve higher acceleration energies that cavities could sustain before a breakdown of the field due to the limiting factors such as field emission and multipacting. Cavities have been usually made out of copper due to its good electrical and mechanical properties. Depending on the size of the cavity, half cells can be fabricated either by deep drawing for smaller cavities or spinning for larger cavities. To assemble a multi-cell cavity, number of half cells are electron beam welded at the iris and the equator edges (Fig. 2.1). After fabrication, multi-cell cavity is mechanically tuned to the required frequency, and stiffening rings are usually added to prevent detuning.

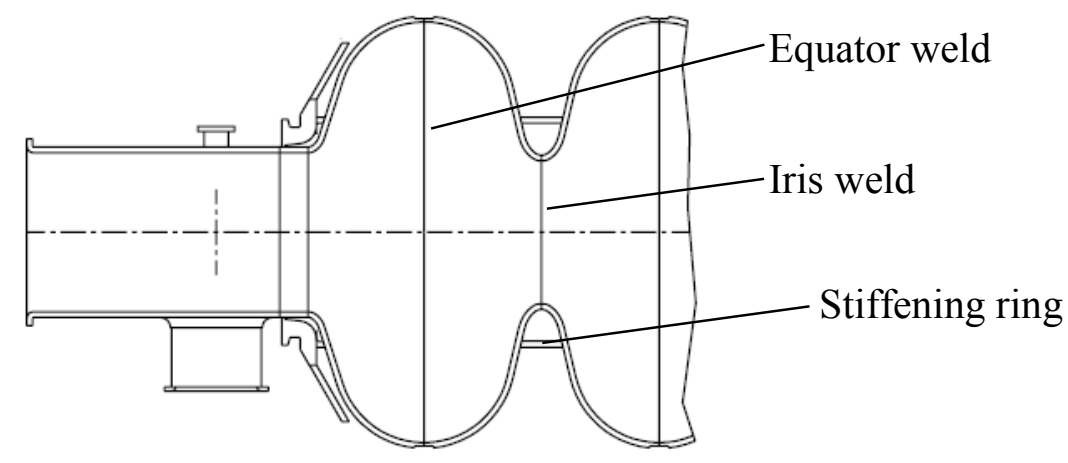

Fig. 2.1 End section of a multi-cell cavity showing iris weld, equator weld, and stiffening ring locations [4]. 
Accelerating gradients of the niobium SRF cavities are currently in the range of 25-30 $\mathrm{MV} / \mathrm{m}$. The theoretical acceleration limit of 50-60 MV/m [4] indicates that further improvement of cavity surface processing techniques could significantly increase the performance of the accelerators. Even though currently used processing techniques have proved to be efficient in removing the surface layers of niobium, the theoretical limit of accelerating field is yet to be achieved. This indicates that alternative methods of processing should be considered to push this boundary further. Recently developed method for plasma processing of $S R F$ cavities [2] might lead to a technique that can further improve the accelerating gradient. However, with the advancement to higher accelerating gradients, various limiting factors, such as multipacting and field emission, were observed. Both of these two limiting factors are responsible for the formation of free electrons inside the cavity. Furthermore, the transfer of energy between the free electrons and the cavity wall could lead to a local temperature increase and formation of normal conducting areas on the superconducting cavity surface leading to further energy losses due to increased resistivity.

In this work, the effects of plasma processing of inner surfaces of niobium $S R F$ cavities and subsequent limitation factors were studied. Specifically, one of the parameters affecting the formation of free particles is the secondary electron emission, which is related to the condition of the cavity surface. Furthermore, as a consequence of the cavity design and required welding procedure, there are several welded locations on the cavity which are located near the areas of significant electrical and magnetic field intensities. These distinct welded areas on the cavity may affect $S E Y$ magnitude compared to the rest of the cavity. An experimental setup capable of measuring the $S E Y$ of niobium surface at different incident angles of impacting electrons was 
developed. In addition, the effect of $\mathrm{N}_{2}$ and $\mathrm{Ar} / \mathrm{O}_{2}$ plasma on $S E Y$ magnitude of all characteristic surfaces located on cavity wall was measured on samples made from cavity grade niobium.

Prior to the discovery of superconductivity [5], accelerators operated at room temperature at which there were significant power losses due to surface resistance of the cavity walls. Significant reduction in resistivity in superconducting state provided the means to reduce the field losses caused by resistivity of copper cavities. The highest critical temperature for superconductivity $\left(T_{c}\right)$ in pure elements was measured in niobium. This made niobium the best material to replace the copper for cavity fabrication. However, any impurity present in the niobium could potentially be the location of normal conducting region, which would cause power losses due to local increase in resistivity. Since the purity of material is essential for operation below the critical temperature, niobium purification technology has improved significantly since it was first used for fabrication of cavities. The measure of purity used in superconducting materials for cavity production is Residual Resistivity Ratio $(R R R)$. This is the ratio of resistivity at $300 \mathrm{~K}$ and just above $T_{c}$, which for niobium is $9.3 \mathrm{~K}$. Impurities usually found in cavities have a critical temperature below that of niobium and potentially could create normal conducting areas which increase the power losses. $R R R$ value of 300 is the typical purity level of niobium used in fabrication of cavities.

\subsection{Performance of accelerating cavities}

Charged particles accelerate by receiving energy from the electric field confined in the accelerator cavities. Acceleration gradient (the axial electric field) is measured in $\mathrm{MV} / \mathrm{m}$ and can be calculated as

$$
E_{a c c}=\frac{V_{a c c}}{d},
$$


where $V_{a c c}$ is accelerating voltage and $d$ is the length of cavity. Quality measure of accelerator cavity is called an Intrinsic Quality Factor ( $Q$ factor). $Q$ factor is given by

$$
Q_{0}=\frac{\omega \cdot U}{P_{c}} \text {, }
$$

where $\omega$ is angular resonant frequency of the cavity, $U$ is the energy stored by electromagnetic field, and $P_{c}$ is dissipated power.

Every fabricated cavity, single- or multi-cell, is tested by gradually increasing the accelerating field and measuring the $Q$ factor until the field breakdown occurs. Electric field achieved before the breakdown is the maximum that cavity can transfer to the travelling bunch of particles. Typical $Q$ curve as a function of the accelerating gradient for niobium cavity is shown in Fig. 2.2.

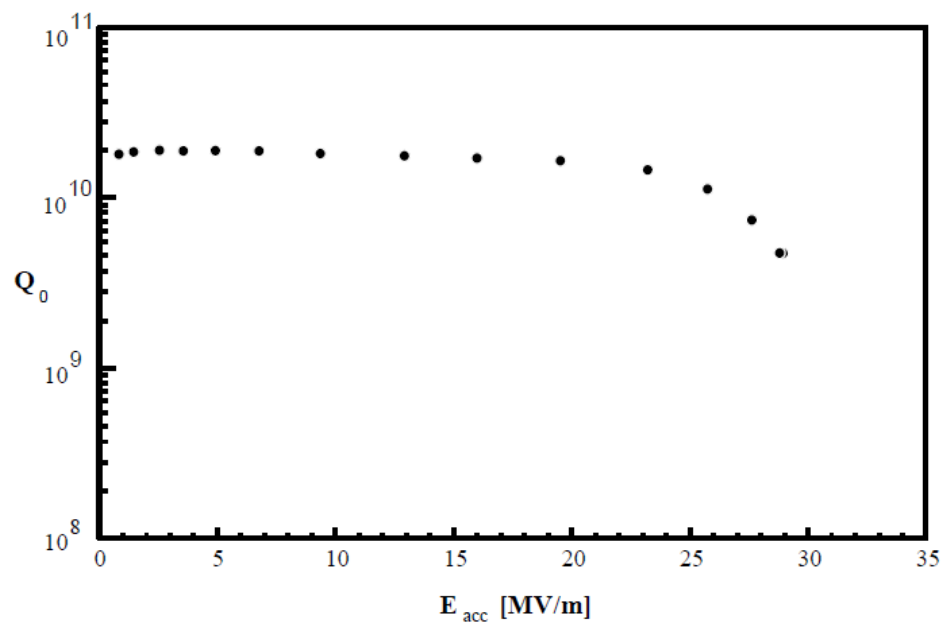

Fig. 2.2 Typical $Q$ factor curve of a multi-cell niobium cavity [4]. 
Increasing the field magnitude that cavities have to contain brought to attention several limiting factors that had to be resolved in order to reach the design goals. Two of the most serious limiting factors that occur in cavities are multipacting and field emissions.

Multipacting is a resonant phenomenon usually occurring in radiofrequency $(r f)$ structures that leads to an increase in number of electrons emitted from the surface. The phenomenon was first described by P. T. Farnsworth in [6] and the term multipacting was coined from multiple impacting describing the behavior of free electrons that are close to surface in an $r f$ field. Two conditions have to be met in order for multipacting to occur. The first condition is the synchronized electron movement in an $r f$ field. The second condition requires the surface to allow electron release due to $S E E$ phenomenon. Synchronization can occur in two specific cases called One Point and Two Point multipacting.

One Point multipacting occurs in the region where the magnetic field is close to uniform and the electric field has normal component of some value that determines the electron impact energy during an $r f$ cycle [7]. Specifically, an electron leaves the surface at the beginning of an $r f$ cycle, during which it follows a certain trajectory only to return to its point of origin at the end of an $r f$ cycle. During flight, the electron gains energy and then transfers it to the surface upon return impact. One Point multipacting was a serious limiting factor for pillbox cavities which was reduced by changing their shape to elliptical. Removal of sharp corners from the cavity geometry reduced significantly the number of locations where One Point multipacting could occur.

Two Point multipacting is usually a characteristic of elliptical cavities and can originate in the area around the equator [8]. In this case an electron leaves the surface and under the influence of an $r f$ field travels to a point which is symmetric to the electron origin across the equator. This 
type of multipacting is very common in elliptical cavities but most of the emitting spots can be mitigated with surface treatment, such are buffered chemical polishing or heat treatment.

Emission of electrons from sharp tips on the surface due to the effect of the applied electric field is called field emission. Field emission is the limiting factor that occurs at high fields in superconducting cavities. The source of an increased field emission can be any type of impurity on the surface of the cavity, embedded during cavity fabrication or handling. It can also be due to a base metal protrusion, created during forming and welding or any type of surface treatment. The protrusion acts like an antenna which under the effect of high electric field, constantly releases electrons inside the cavity. When a steep decrease in $Q$ factor at high electric field is observed, it usually signals presence of a significant increase in surface emitted electrons. Released electrons can be accelerated by cavity fields and upon return to the surface could transfer gained energy into heat, $x$-rays, and more electrons due to $S E E$.

The best approach to reduce field emission is to fabricate as clean surface as possible. To achieve this, a number of different cleaning and handling procedures have been developed [4]. Currently, two main cleaning procedures are used, Buffered Chemical Polishing $(B C P)$ and Electro-Chemical Polishing $(E C P)$. To complement the effect of cleaning other procedures have been developed and used that precede or follow $B C P$ or $E C P$. Baking of cavities and High Pressure Rinsing $(H P R)$ are among commonly used supplemental cleaning techniques. In addition, handling and assembly of cavities is performed in clean rooms. Implementation of these techniques has given good results and today nine cell cavities regularly achieve acceleration of $25 \mathrm{MV} / \mathrm{m}$ before field breakdown. However, there were cases where cavities suffer breakdown sooner and upon inspection, some low field emitter was regularly discovered. Additional cleaning was usually able to fix this problem and improve the accelerating gradient. 
Sometimes the cleaning has to be done in-situ and for that purpose high power processing is usually used [9]. When a field emitter is discovered, an excess of $r f$ power is delivered into the cavity to process it. The high field current emitted around the impurity ionizes the gas around it and creates a plasma discharge that removes the impurity from the surface. Following the change in $Q$ factor with an electric field increase gives an insight into the overall state of the surface. In order to find the specific location of field emission or any type of losses on the cavity, surface temperature measurement is an accurate technique of determining the position of local heat sources [9]. Multiple thermometers are attached to the cavity in order to measure the temperature distribution across the surface. To obtain the accurate measurements, good contact between the surfaces of thermometer and the cavity has to be maintained. Temperature gradient field obtained will provide the location of field emitting spots on the cavity surface (Fig. 2.3). Once the location of field emitters are known, the cavity can be dissected and examined to their source. In Fig. 2.3, micrograph taken by Scanning Electron Microscope (SEM) revealed a copper particle imbedded on the surface [9].

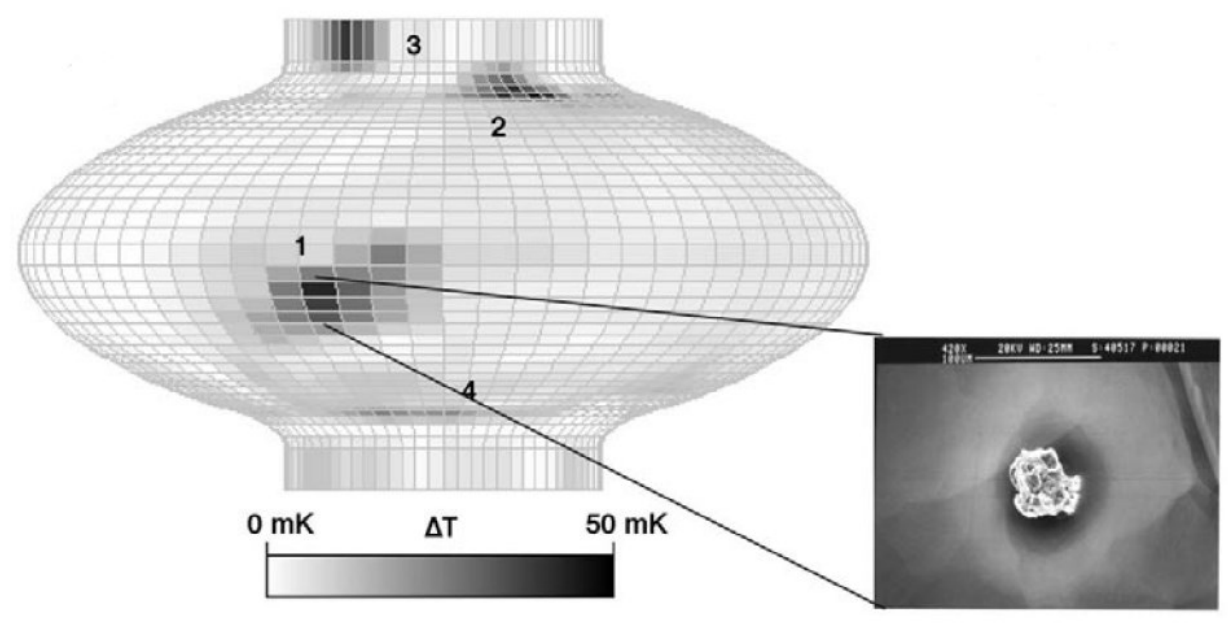

Fig. 2.3 Temperature gradient of a cavity with a local temperature increase at $1,2,3$, and 4 [10]. 
Energy transfer from electrons to cavity surface can cause local transition from superconducting to normal conducting state if the field emission is significant. Resistivity grows exponentially with the temperature and as a consequence, normal conducting patches now become the locations for further power dissipation on the surface which eventually leads to the "quenching" of the cavity. A quenching condition ensues when the cavity is no longer able to sustain the stable electric field.

The most severe limiting factor in cavities is field emission which becomes significant at high accelerating gradients when $Q$ factor of the cavity starts to decrease. Regardless of how well the cavity is fabricated and treated with current processes, when a sufficiently high electric field is applied, field emission will cause the breakdown and quenching of cavities. Even though the current multi-cell cavities now regularly achieve $25 \mathrm{MV} / \mathrm{m}$ gradients and single cell experimental cavities have achieved gradients of up to $40 \mathrm{MV} / \mathrm{m}$ [11] that is still below the theoretical maximum for niobium, which is around $50 \mathrm{MeV} / \mathrm{m}$ [12].

\subsection{Secondary electron emission}

As the accelerating gradient of cavities continue to increase towards theoretical limit, both multipacting and field emission will become significant inside the cavity. These physical processes increase the number of the free electrons inside the cavity thus limiting the performance of the accelerating cavities. Free electrons released from field emitters and multipacting locations absorb the energy during an $r f$ cycle and impact the surface releasing the additional electrons. The electron build-up ultimately causes the breakdown of the field inside the cavity. Free electrons that eventually impact the surface, transfer part of the energy to the surface. This excess energy can cause the release of additional electrons through Secondary 
Electron Emission (SEE) process. To reach the theoretical accelerating gradient it is necessary to reduce the secondary electron emission from the cavity surface to the lowest possible value.

Secondary electron emission is a process during which additional free electrons are formed and as such it is relevant for the cavity and accelerator operation. It was first discovered by Austin and Starke [13] in 1902 when they observed that exposure of metal surface to electron beam initiated release of more electrons than it was receiving. Higher predisposition of surface to SEE promotes the release of free electrons leading to early field breakdown in the cavity. When electrons of certain energy level hit the surface of a solid they can penetrate the surface potential barrier and transfer energy in a series of collisions with atoms of the surface. These impacting electrons are called primary electrons. During the collision process, primary electrons can transfer enough energy to the surface causing the release of additional electrons that could leave the surface of the solid under certain conditions. The electrons leaving surface are called secondary electrons. Once secondary electrons leave the surface, they absorb the energy during an $r f$ cycle and impact the surface. Consequently, this creates an avalanche effect that eventually breaks down the accelerating field inside the cavity. To quantify the emission of secondary electrons, a magnitude defined as a ratio of the number of secondary electrons over the number of primary electrons was introduced and named a Secondary Electron Yield (SEY). A SEY magnitude larger than 1 indicates the increase in the number of free electrons. The cavity wall with a high $S E Y$ magnitude will achieve quenching conditions sooner. 


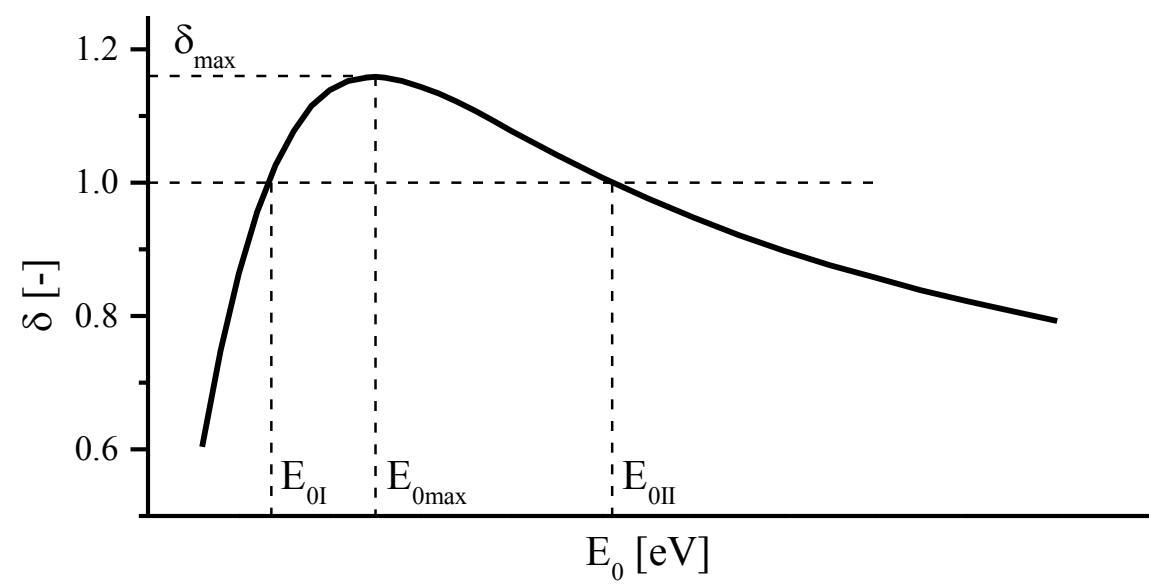

Fig. 2.4 Typical $S E Y(\delta)$ curve as a function of primary electron beam energy $\left(E_{0}\right)$ with characteristic points.

Experimental measurements done in the past have shown that there is a typical curve that describes the $S E Y(\delta)$ as a function of primary energy of the impacting electrons $\left(E_{0}\right)$. A typical $S E Y$ curve has three characteristic points that are of interest for any metal surface (Fig. 2.4). The first crossover point is a point where $S E Y$ equals $1(\delta=1)$ at the corresponding energy $E_{0 I}$, next is the point of maximum yield $\delta_{\max }$ which occurs at $E_{0 \max }$ energy, and the second crossover point is where $\delta=1$ again for $E_{\text {OII }}$ energy. If the maximum $S E Y$ has a value larger than 1 then the number of electrons leaving the surface will be larger than number of electrons impacting it. For accelerator cavities, the increasing number of free electrons absorb increasing amounts of energy intended for the accelerated particles. When the free electrons start absorbing energy intended for the accelerated particles, the reduction of the $Q$ factor is observed. 


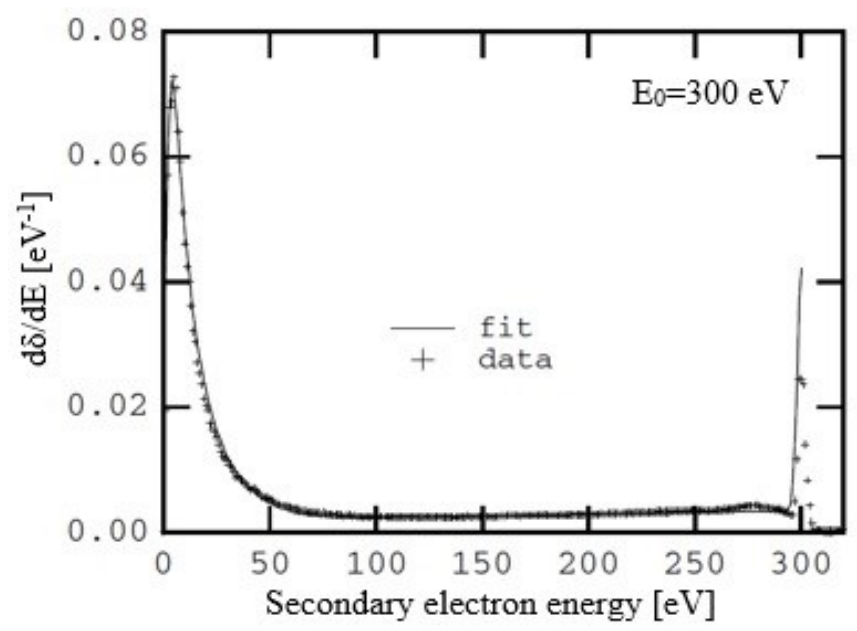

Fig. 2.5 Secondary electron energy distribution for stainless steel and $E_{0}=300 \mathrm{eV}$ at normal incident angle [14].

Furthermore, electrons leaving the surface of a cavity wall have an energy distribution in the range from $0 \mathrm{eV}$ to the energy of the primary electrons $\left(E_{0}\right)$. A typical energy distribution curve $(E D C)$ has three characteristic sections that describe the energy levels of the electrons leaving the surface (Fig. 2.5). The first section is a peak in the lower energies that represents the quantity of true secondary electrons. Electrons that received the energy from the collision with the primary electrons and that consequently left the surface of the solid are called true secondary electrons. True secondary electrons are considered to have the energy levels in the range between $0 \mathrm{eV}$ and $50 \mathrm{eV}$ [15]. The second section of the $E D C$ is the flat part of the curve from $50 \mathrm{eV}$ to the energy of the primary electrons. Electrons in that energy range are considered to be inelastically reflected primary electrons. These electrons impact the surface, transfer part of their energy and then leave the surface. The third section of the curve is the sharp peak that is formed at the energy level of the primary electrons and these electrons are called elastically reflected primary electrons. 


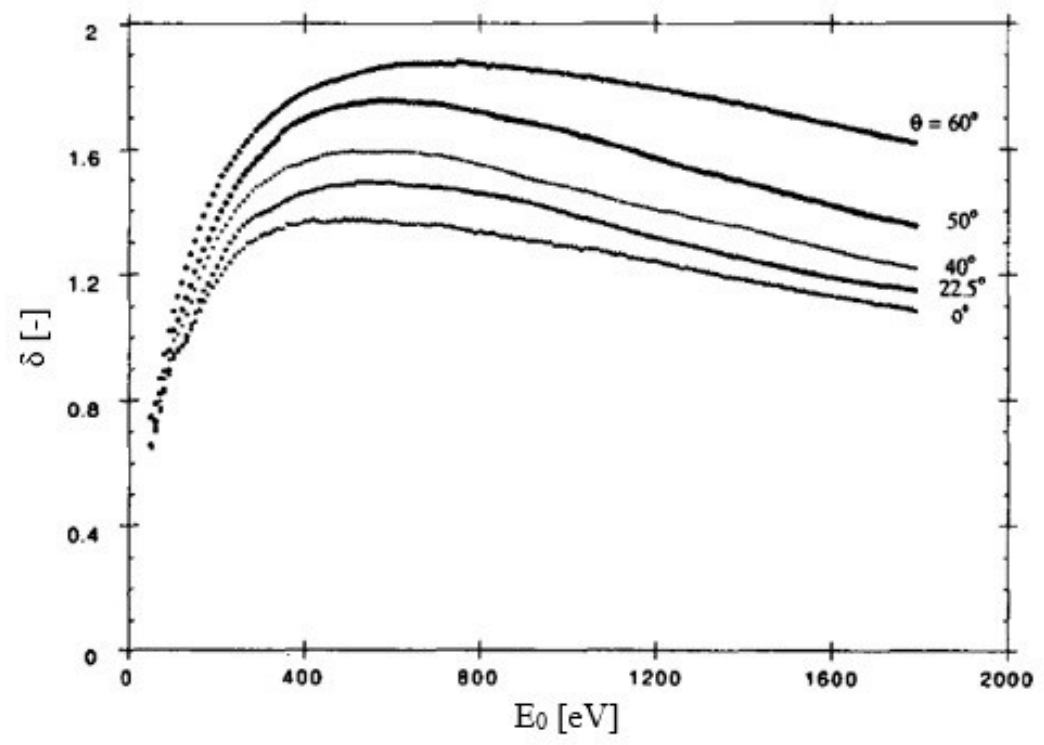

Fig. 2.6 $S E Y(\delta)$ as a function of $E_{0}$ and increase in incident angle $(\theta)$ measured on a molybdenum sample [16].

The $S E Y$ is not only a function of the energy of primary electrons but also a function of the incident angle $(\theta)$ of primary electrons. The angle between impacting electrons and normal to the surface is called an incident angle of the primary electrons. Experimental results have shown that the overall yield increases with the increase in the incident angle [16]. Furthermore, the energy level at which maximum yield occurs was increased with the incident angle (Fig. 2.6). Determining the $S E Y$ curves as a function of incident angles was needed, since the incident angle of primary electron impact on the surface of the cavity is a probabilistic event.

\subsection{The effect of cavity microstructures on $S E Y$}

Half-cells of a cavity are electron beam welded at equator and iris edges. During welding procedure, significant amount of heat was induced in niobium. It was reported that $S E Y$ was a function of magnitude and duration of the heat treatment [17]. Consequently, the welding 
procedure might change the $S E Y$ characteristic in the weld area compared to the $S E Y$ of the surface not affected by welding.

After welding, three characteristic zones in and around the weld are differentiated, namely the weld zone $(W Z)$, the heat affected zone $(H A Z)$, and the base metal (BASE). Differences between the zones can be observed in their microstructure.

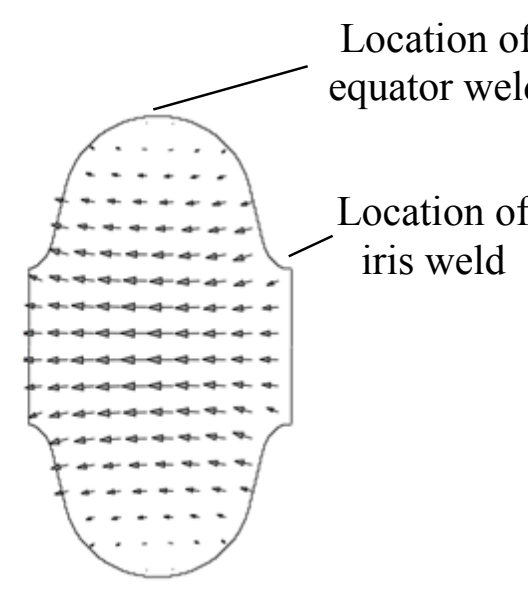

(a)

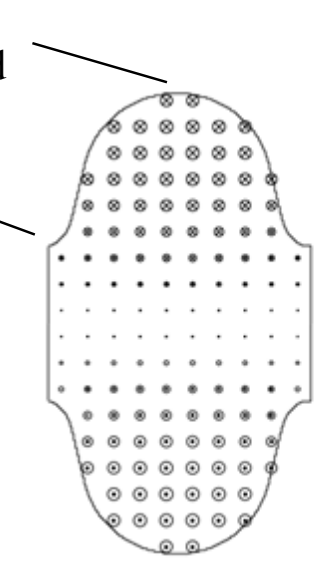

(b)

Fig. 2.7 Distribution of (a) electric and (b) magnetic field in a $S R F$ elliptical cavity.

In addition to changes in microstructure, high electric and maximum magnetic fields are present near the welded areas of the cavity (Fig. 2.7). Different surface states in the weld areas of the cavity combined with high intensity fields may create specific conditions that affect the $S E Y$ property of the material. An extensive literature review indicates that no such measurement has been made thus far.

\subsection{Scope of the dissertation}

In this work, the developed experimental setup has been used to test the $S E Y$ of niobium samples under various conditions. Two groups of samples were used that represent the 
characteristic surfaces found on the cavity walls. The first group of samples was made of cavity grade niobium. The second group of niobium samples was made from the same material but it included the weld joint, simulating the surface near equator and iris of the cavity. Welded samples were made using the same welding parameters used for cavity fabrication to simulate the surface conditions. Furthermore, the experimental setup allows the measurement of angular dependence of the $S E Y$. Since the incident angle has a large impact on $S E Y$, the range of incident angles for which the measurements were possible was very important.

Both groups of samples were exposed to plasma and all measurements were repeated to determine the effect on the niobium surface. Recent work has provided the method of applying plasma as a technique for post fabrication treatment of cavities [2]. The experimental setup developed by J. Upadhyay et al. [2] was able to remove the surface layers of niobium from curved samples by processing them in a plasma created in argon/chlorine gas mixture. Using the described method, plasma processing of the curved surfaces of $S R F$ cavities is possible with gases commonly used for removing the non-metallic surface layers of contamination. Secondary electron yield on samples with characteristic microstructures present in SRF cavities were determined before and after plasma processing in nitrogen and argon/oxygen gases.

The work presented here provides a comprehensive work on the $S E Y$ of niobium used in accelerating cavity fabrication. The work provides results for characteristic locations, different incident angles of primary electrons, as well as the effect of plasma processing on a niobium surface. 


\section{CHAPTER 3}

\section{LITERATURE REVIEW}

Since the formulation of a secondary electron emission process, there has been an ongoing effort to describe the behavior of different surfaces when exposed to the impacting electrons [15]. To quantify the $S E E$, a magnitude called the secondary electron yield was defined as the ratio of emitted and impacting electrons [18]. The aim of this chapter is to provide an overview of the secondary electron emission topic and its significance related to the particle accelerator application. The chapter is divided into sections describing the available SEY measuring techniques, derived numerical models of $S E Y$, most commonly used techniques for reducing the $S E Y$, and an overview of the methods used for processing of niobium $S R F$ cavities.

\subsection{SEY measurement techniques}

Secondary electron yield is defined as the ratio of the emitted electrons current and the impacting electrons current. The free electrons focused into a beam impact the surface of the sample, triggering the release of the electrons. The current of the electrons that were emitted by the surface can be measured with two different approaches. The first, is to measure the current on the sample, to determine the quantity of impacting and emitted electron currents. The current of the emitted electrons can be determined as a difference between the current of the sample and the current of the electron beam impacting the sample. In the second method, a current is measured on the collector that completely envelopes the sample, which comes from the absorbed electrons emitted from the surface of the sample. Each of the methods has specific advantages over the other, and the choice between the methods is usually based on the experimental goals, but can 
also depend on the available space at the measurement location for in-situ applications. The details of both $S E Y$ measurement techniques with corresponding advantages and limitations are discussed below.

\subsubsection{Sample current method}

When a sample current method is used, the $S E Y$ is determined by measuring the sample current and the primary electron beam current. The relation between the $S E Y(\delta)$, the sample current $\left(i_{s}\right)$, and the primary electron beam current $\left(i_{p}\right)$ is defined as

$$
\delta=\frac{i_{p}-i_{s}}{i_{p}}=1-\frac{i_{s}}{i_{p}} .
$$

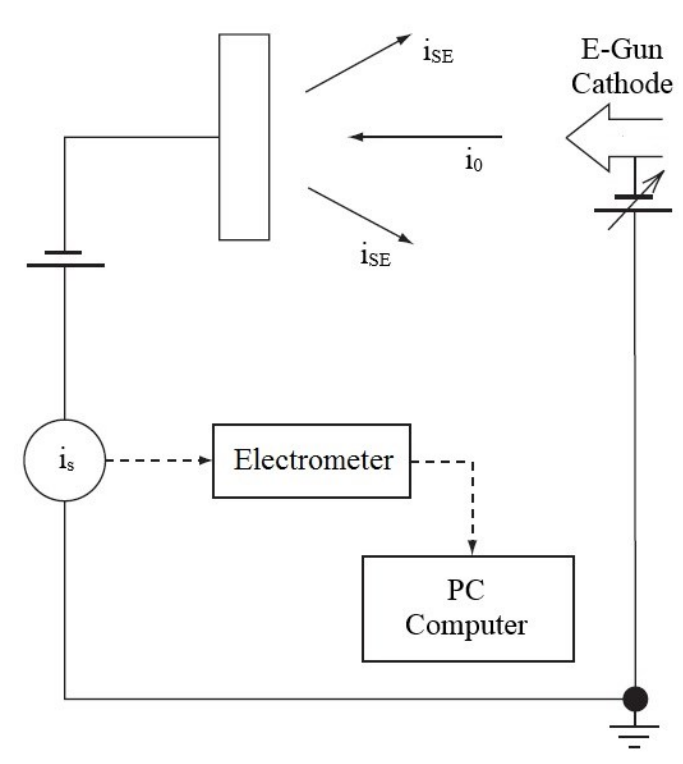

Fig. 3.1 Schematic of the sample current method [19].

The current of the emitted electrons $\left(i_{S E}\right)$ is determined indirectly as the difference between the electron beam and the sample currents. Specifically, the sample current is measured by 
positioning the sample in front of the electron gun at normal incidence angle of a primary electron beam. The sample should be biased to a steady negative potential to repel emitted electrons leaving the surface and tertiary electrons that are formed on the surrounding boundaries of the experimental setup [20-22]. Tertiary electrons are formed when the electrons emitted from the surface of the sample impact the surrounding walls of the experimental setup. By negatively biasing the sample, an erroneous current reading due to the tertiary electrons returning to the surface is avoided. A simple schematic of the sample current method is shown in Fig. 3.1.

The current of a primary electron beam can be measured with two different methods. In the first method, the sample located in front of the electron gun is biased at a high positive potential to retain all the electrons that are leaving the surface [21,22]. With the sample at high positive potential, the current measured on the sample is considered the primary beam current. However, not all electrons can be captured if the energy of the primary electron beam is larger than the bias of the sample. If the energy of the elastically and inelastically reflected electrons is high enough it cannot be retained by the positively biased surface. All electrons with the energy levels higher than the positive bias can leave the surface of the sample, thus creating a discrepancy between the actual and measured beam current. In the second method, a Faraday cup with a small aperture can be placed in front of the electron gun to determine the beam current. The aperture is centered to allow the flow of electrons inside the Faraday cup, while the aperture size allows only few electrons to leave. The current measured in the Faraday cup is considered the primary electron beam current. Maintaining the current magnitude of the electron beam across the energy range of the primary electrons is one of the parameters that are needed to be controlled during the $S E Y$ measurement. It is necessary to measure the beam current at each energy level of primary 
electrons, since keeping it uniform can be challenging [22,23]. This significantly increases the measurement time for a large number of primary electron beam energy values.

During the $S E Y$ measurement, the sample potential was set to a negative value to reflect the electrons returning to the surface of the sample. This ensures that the current measured on the sample is only due to the electrons leaving the surface. However, the negative potential of the tilted sample becomes deflective thus not allowing the measurement of $S E Y$ at different incident angles of the primary electrons. The negative potential on the inclined sample can change the spot where the primary electrons are impacting the surface. Low energy primary electrons can be deflected enough to completely evade the surface of the sample.

\subsubsection{Collector current method}

In the collector current method, the $S E Y$ is determined by measuring the sample current $\left(i_{s}\right)$ and collector current $\left(i_{c}\right)$. The relation between the $S E Y$, sample current and the collector current is defined as

$$
\delta=\frac{i_{c}}{i_{c}+i_{s}}=\frac{i_{c}}{i_{p}} .
$$

Primary electrons pass through the aperture on the collector and impact the surface of the sample. Electrons emitted from the surface are captured by the collector. In order to retain the electrons that reach the surface of the collector, a positive potential is applied to the collector [24]. The sample is usually grounded or kept at a very low negative potential to make sure that emitted electrons leave the surface. The primary electron beam current is indirectly obtained as the sum of the measured collector and sample currents. The $S E Y$ curve is determined by increasing the energy of the primary electrons and recording the measured currents on the collector and the sample. The collector current method can be used to obtain the $E D C$ of the reflected electrons by applying a retarding potential on the collector and incrementally reducing 
it and recording the current values at the sample and collector. The $S E Y$, at different incident angles, can be determined based on the assumption that all the emitted electrons can be captured. The simple schematic of the collector current method is shown in Fig. 3.2.

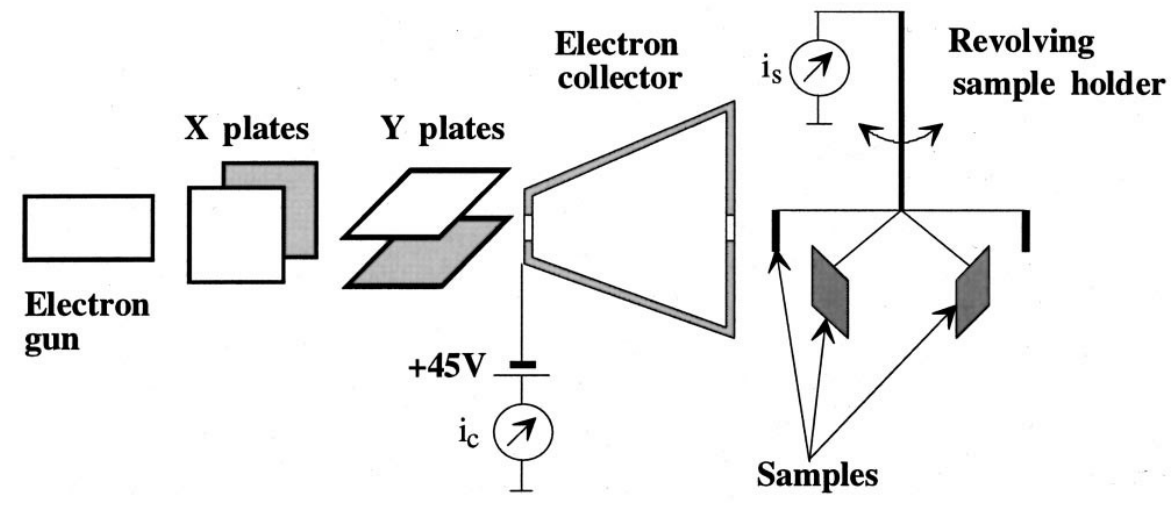

Fig. 3.2 Schematic of the collector current method [24].

Even though the measurements of the sample, collector, and the beam currents may appear straightforward, accurate measurement can still be affected by technical difficulties. Some of these difficulties are discussed in [25], while the details of difficulties encountered in measuring SEY using sample current method along with potential mitigation techniques are discussed in [26].

\subsection{Numerical models of $S E Y$}

High dependence of the $S E Y$ on the surface condition is the cause of limited reproducibility observed in the experimental results. The majority of experimental setups are capable of measuring $S E Y$ only at the normal incident angle of primary electrons. The function of $S E Y$ at normal incidence of primary electrons is frequently described by a semi-empirical law [27], and sometimes is modeled by completely empirical functions [28]. A strong dependence of $S E Y$ 
results on the incident angle of primary electrons should not be overlooked. An increase in the incident angle of primary electrons causes the increase in the maximum $S E Y$ value as well as the energy at which the maximum yield occurs. Ever since the formulation of $S E E$, numerous models have been developed to describe the experimental data. Several models that describe the SEY at normal and other than normal incident angles have been presented [27-30].

The most commonly used equations that describe the $S E Y$ for normal incidence of primary electrons were derived from a semi-empirical law of $S E Y$ [27,29]. In these two reports, a similar function was derived, but with different parameters, which were obtained based on the experimental results, Eqs. (3.3) and (3.4),

$$
\begin{aligned}
& \frac{\delta}{\delta_{\max }}=1.11 \cdot\left(\frac{E_{0}}{E_{0 \max }}\right)^{-0.35} \cdot\left(1-e^{\left(-2.3 \cdot\left(\frac{E_{0}}{E_{0 \max }}\right)^{1.35}\right)}\right), \\
& \frac{\delta}{\delta_{\max }}=1.28 \cdot\left(\frac{E_{0}}{E_{0 \max }}\right)^{-0.67} \cdot\left(1-e^{\left(-1.614\left(\frac{E_{0}}{E_{0 \max }}\right)^{1.67}\right)}\right) .
\end{aligned}
$$

An example of an empirical model of $S E Y$ is described in Ref. [30],

$$
\frac{\delta}{\delta_{\max }}=1.379 \cdot \frac{1-e^{\left(-1.844\left(\frac{E_{0}}{E_{0 \max }}\right)^{1.35}\right)}}{1.844 \cdot\left(\frac{E_{0}}{E_{0 \max }}\right)^{0.35}}
$$

Another example of an empirical model is given in Ref. [28],

$$
\frac{\delta}{\delta_{\max }}=\left(\frac{E_{0}}{E_{0 \max }}\right) \cdot \frac{p}{p-1+\left(\frac{E_{0}}{E_{0 \max }}\right)^{p}},
$$

where $p>1$ is a fit parameter. The current models are functions of maximum $S E Y$, energy of primary electrons at which the maximum $S E Y$ is observed, and fitting parameters. The 
independent variable in these models is always the energy of the primary electrons. The above models can be fitted to experimental data by adjusting the fitting parameters and applying the measured maximum SEY and corresponding energy. The accuracy of the presented models in Eqs. (3.3-3.6) was tested by comparing it to the $S E Y$ results for niobium (Fig. 3.3). The numerical parameters of each equation are kept as they were derived, except for the Eq. (3.6) where the best fit was obtained for the fit parameter $p=1.434$. Input parameters of the equation were maximum $S E Y$ and energy at which maximum yield occurred taken from [3], for niobium samples after $B C P$. It was observed that the best fit was obtained from Eqs. (3.3) and (3.5).

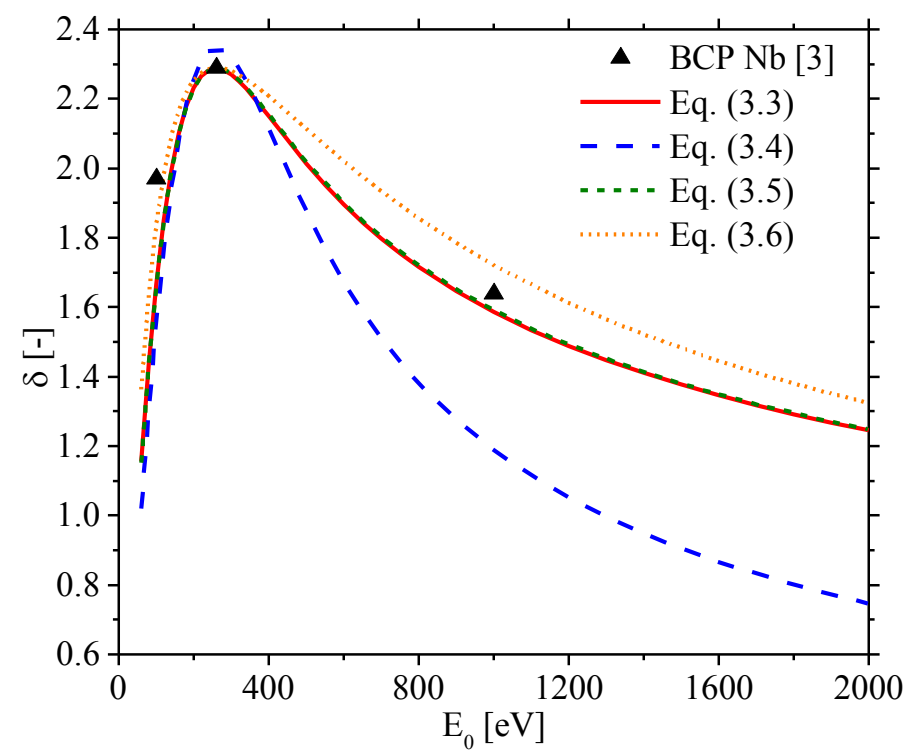

Fig. 3.3 $S E Y$ as a function of $E_{0}$, showing the agreement of different models represented by Eqs. (3.3-3.6) with the experimental results of niobium samples [3].

The effect of the incident angle of primary electrons on the $S E Y$ was observed early in the experimental studies of $S E E$ [31]. It was observed that $S E Y$ values increase with the increase of the incident angle of primary electrons. In addition, it was observed that the energy level at 
which the maximum $S E Y$ was detected increased as well (Fig. 2.6). The overall increase in $S E Y$ values at all energies can be related to the depth at which the secondary electrons are formed [32]. The most commonly used assumption is that if the primary electron of a certain energy at normal incidence stops at a depth $X_{m}$ from the surface, then the same electron impacting the surface at an angle $\theta$ will stop at $X_{m} \cos (\theta)$ depth (Fig. 3.4). Secondary electrons formed along the path of the incident primary electrons are closer to the surface and have a higher probability to leave the substrate, which leads to the increase in $S E Y$.

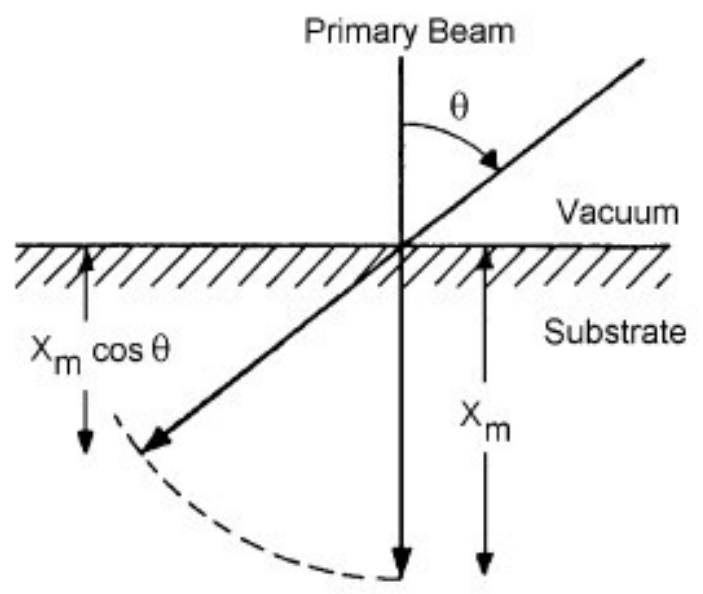

Fig. 3.4 Schematic representation of the commonly assumed model of the secondary electron formation depth as function of incident angle [32].

There are several approaches commonly used to model the effect of an incident angle of primary electrons on $S E Y$. The models formulated in [31] and [27] describe the increase of $S E Y$ with the increase in incident angle of primary electrons. However, the increase in energy level at which the maximum yield is observed, is not accounted for. Vaughan [30] suggested that better fitting to the experimental results might be obtained if maximum $S E Y$ and corresponding energy 
of primary electrons are adjusted first by empirical correction formulas, and then calculating the SEY curve based on these corrected values.

Currently used SEY models are based on either semi-empirical or entirely empirical formulation. Semi-empirical models attempt to find a correlation between the properties of materials and SEY by performing extensive measurements on the large number of different elements and compounds. The analysis of the experimental data has provided a database of parameters for modeling the $S E Y$ of large number of elemental surfaces and compounds [27,29]. However, due to the high influence of the surface layer composition, the measured SEY can significantly differ from the database results.

\subsection{Techniques for reducing the $S E Y$}

Formulating the efficient techniques for controlling the $S E Y$ has been a topic of research ever since the discovery of secondary electron emission. Over the years, several methods were developed for applications in accelerators and the aim of each one was the reduction of $S E Y$ magnitude $[3,17,33,34]$. The methods were formulated in order to reduce the formation of free electrons inside of the beam tubes, waveguides, and accelerating cavities, which always have a detrimental effect on the traveling beam and pressure level inside the accelerator. In this section, several techniques that are currently used are presented and their effect on $S E Y$ is described.

Described techniques include thin film deposition, heat treatment, electron beam irradiation, and plasma processing. However, due to the specific requirements of the accelerating $S R F$ cavity, some of these methods are not applicable to the surface of the cavity while the others would require adaptation and special tools which would make them inefficient. 


\subsubsection{Influence of thin film deposition on $S E Y$}

Thin film deposition method is a process in which a thin film of materials is deposited on the substrate, where a thin film is considered to be a layer of material ranging from less than a nanometer to several micrometers in thickness. The purpose of thin films formed by either chemical or physical deposition mechanisms is to change the surface properties of the base material. Thin film deposition method has received considerable attention in various industries such as metal, pharmaceutical, and semiconductor. Deposition technology was successfully applied to flat surface samples of various accelerator components. The tests of the effect of various thin films on $S E Y$ was number of researchers [33,35,36]. Although several surface coatings showed the capability to reduce the $S E Y$ of the base, currently this technology is not applicable to accelerating cavities. The difficulties arise from the complex shape of the cavity which makes uniform deposition of thin films challenging, which can affect superconducting state across the surface of the cavity.

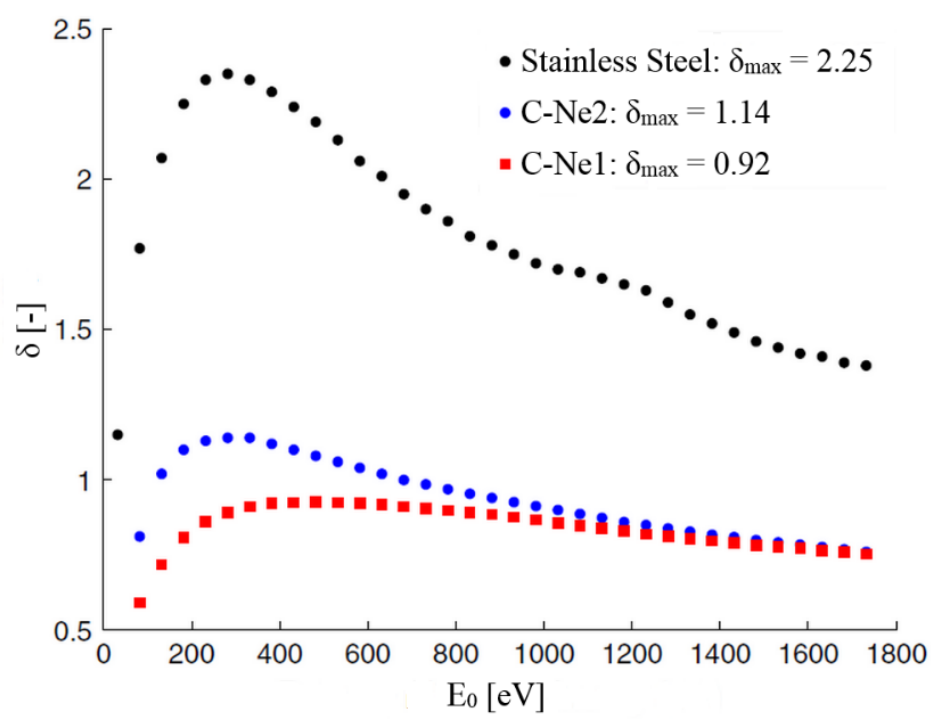

Fig. 3.5 SEY as a function of $E_{0}$, showing the effect of $100 \mathrm{~nm}$ carbon thin film deposited on stainless steel base [33]. 
It was determined that carbon has a low value of maximum $S E Y$ [29]. Thin carbon film of approximately $100 \mathrm{~nm}$ has been deposited on stainless steel base and the positive effect on $S E Y$ was determined [33]. A carbon deposited surface exhibited a significant decrease in $S E Y$ compared to the surface of uncoated stainless steel (Fig. 3.5). The exhibited decrease in $S E Y$ magnitude indicates that the primary electrons of energy range up to $1800 \mathrm{eV}$ penetrate the surface less than $100 \mathrm{~nm}$ in depth. In another test, using a copper as a base, a carbon thin film of $20 \mathrm{~nm}$ thickness was deposited on the surface [35]. The results indicate that the deposited layer of carbon reduces the $S E Y$ of base copper surface. However, the reduction in the maximum value of $S E Y$ was not significant leading to a conclusion that part of the primary electrons were still able to go through the $20 \mathrm{~nm}$ of carbon thin film and reach the copper substrate (Fig. 3.6).

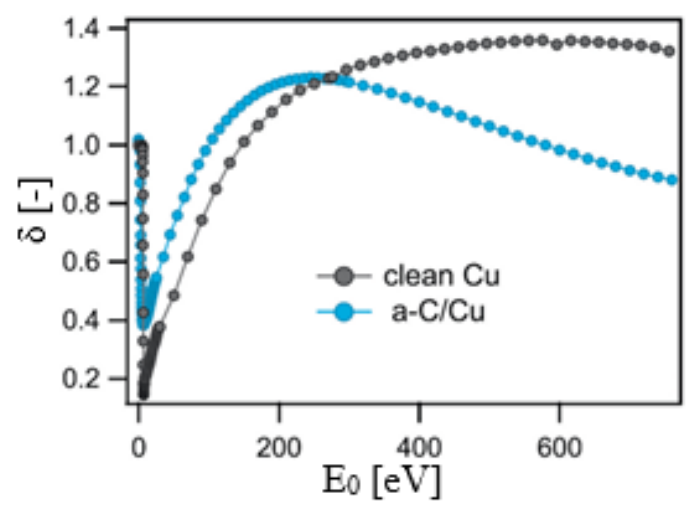

Fig. 3.6 SEY as a function of $E_{0}$, showing the effect of $20 \mathrm{~nm}$ carbon thin film deposited on the copper base [35].

The effect of niobium nitrides and carbides deposited on a niobium base on $S E Y$ has been examined [36] and the results were presented in Fig. 3.7. The surface of the niobium samples used in those tests were cleaned by ion sputtering. Sputtering is a process in which gas ions 
impact the surface and remove the particles embedded on the surface. A sputter cleaned surface of niobium was used as a substrate for deposition of niobium nitride and carbide thin films of 14 $\mathrm{nm}$ thickness. When compared to the $S E Y$ of sputter cleaned surface of niobium, surfaces with thin films of niobium nitrides and carbides have shown an increase in $S E Y$ (Fig. 3.7 (a)). Additionally, after the $S E Y$ measurements were performed, the samples were exposed to air at atmospheric pressure for one hour. Following the exposure, measurements were repeated and the increase in $S E Y$ was observed on all samples (Fig. 3.7 (b)). It can be concluded that surfaces cleaned or deposited in the vacuum were reacting with the air during exposure forming the layer of oxides on the surface. After air exposure, $S E Y$ of the examined surfaces was reduced by electron beam irradiation (Fig. 3.7 (c)).
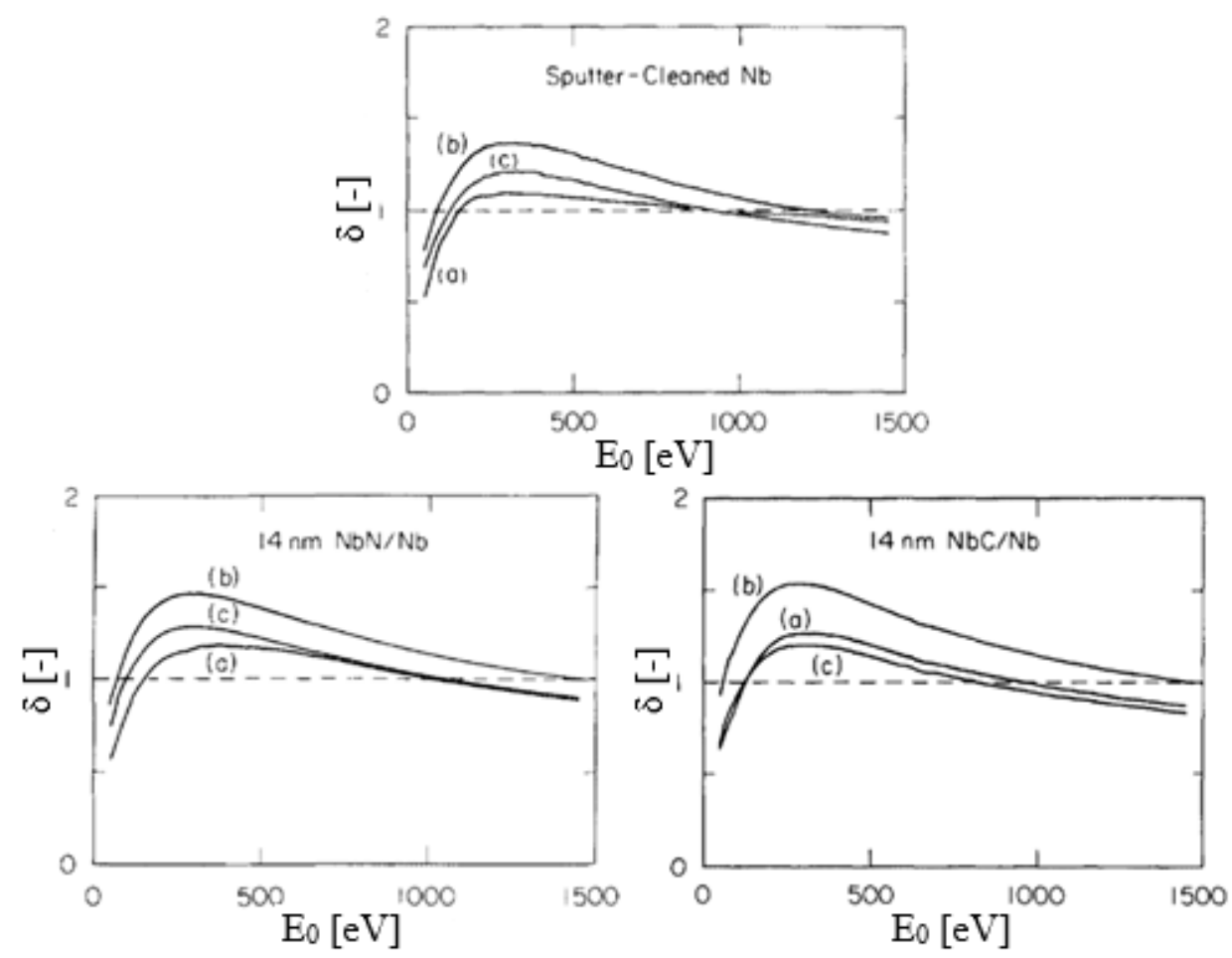

Fig. 3.7 $S E Y$ as a function of $E_{0}$, showing the effect of $14 \mathrm{~nm}$ of $\mathrm{NbN}$ and $\mathrm{NbC}$ deposited on (a) clean $\mathrm{Nb}$ sample. $S E Y$ increases (b) after air exposure, which was reduced with (c) the electron beam irradiation [36]. 
The thin film deposition technology showed the capability of reducing the $S E Y$ of the surface when low yield materials were used and sufficient thickness of the layer was formed. However, there are several difficulties which prevents the application of this technology to accelerating cavities. First, the complex shape of the cavity makes uniform deposition of the thin films challenging. Second, most of low yield materials used for thin films either cannot be superconductors or have a lower critical temperature of superconductivity than niobium. These difficulties have limited the use of thin film deposition for the purpose of $S E Y$ reduction to normal conducting components of accelerators, such are beam tubes and waveguides.

\subsubsection{Influence of heat treatment on $S E Y$}

During the exposure to elevated temperatures, gas impurities trapped on the surface of the material start slowly evaporating. The effect of the heat treatment on $S E Y$ of the $1.5 \mu \mathrm{m}$ thin film of niobium deposited on copper base was reported in Ref. [17]. First, the $S E Y$ was measured after one hour of heat treatment at increasing temperatures (Fig. 3.8 (a)). The reduction of the SEY was first observed after the temperature was raised to $120{ }^{\circ} \mathrm{C}$. Further reduction in SEY was observed with the increase in temperature. The secondary electron yield was determined at elevated temperatures of the sample, except for the first and last measurement which were performed at the ambient temperature of the sample. For the last measurement, sample was kept at $350{ }^{\circ} \mathrm{C}$ for one hour and then left to cooldown for six hours before performing the $S E Y$ measurement.

A reduced $S E Y$ indicates that the evaporated gas impurities were not reabsorbed by the surface after the sample returned to the room temperature since they were probably removed by the vacuum system. Another sample was baked at $150^{\circ} \mathrm{C}$ for 26 hours (Fig. 3.8 (b)). During the heat treatment, the $S E Y$ was measured after 10, 50, and 100 minutes, 4 hours, and 26 hours. After 
baking the sample for 4 hours there was no further reduction in the measured $S E Y$. Secondary electron yield continued to decrease with increase 4 in heat treatment temperature, reaching the evaporating temperature of impurities still present on the surface of the sample. An additional beneficial effect of the heat treatment can also be observed in the $S E Y$ measured on niobium sample [37]. The secondary electron yield was measured at 25 points on $25 \mathrm{~mm}^{2}$ area before and after annealing at $700{ }^{\circ} \mathrm{C}$, indicating that heat treatment also contributed to the uniformity of $S E Y$ magnitudes across the examined surface. The observed uniformity of $S E Y$ could have occurred due to the redistribution of surface oxides during and removal of localized gas impurities during the annealing process.

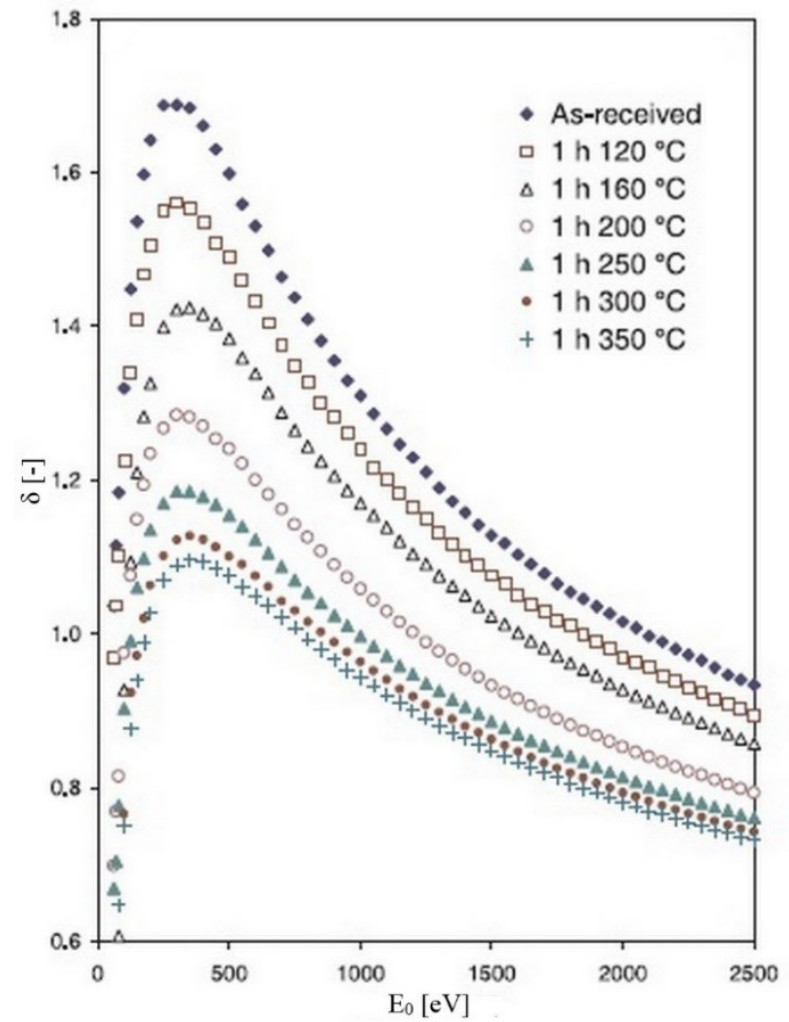

(a)

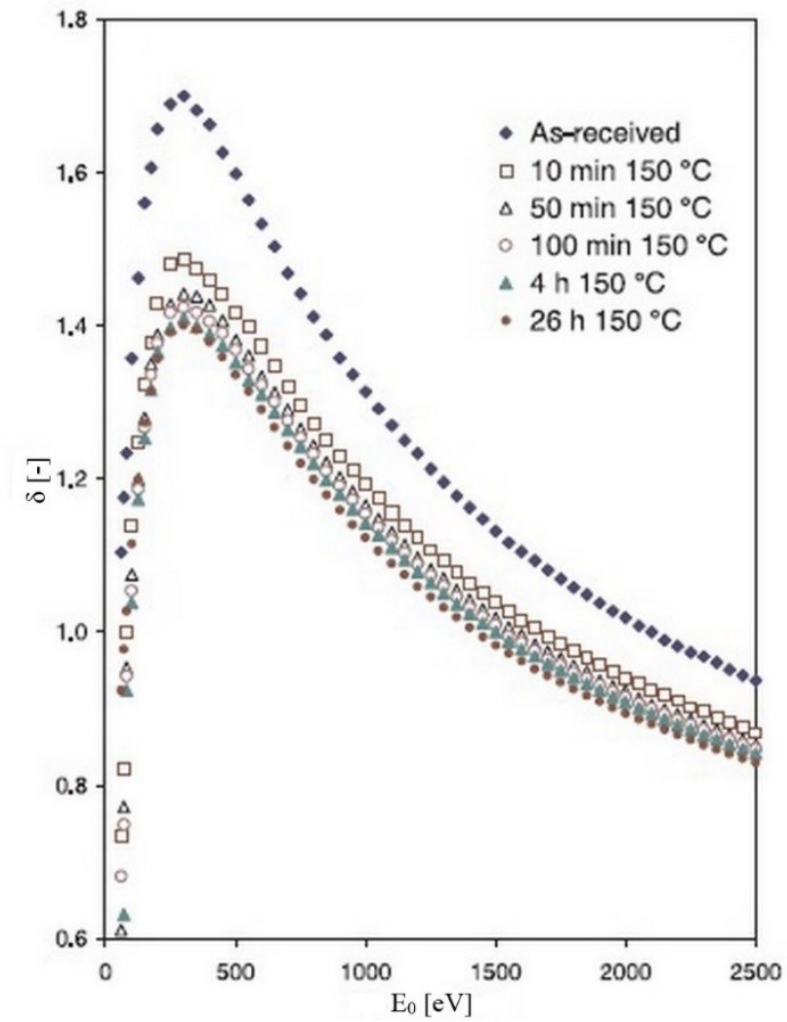

(b)

Fig. 3.8 $S E Y$ as a function of $E_{0}$ of niobium thin layer deposited on copper base, showing the effect of heat treatment at (a) different temperatures and (b) different duration at $150{ }^{\circ} \mathrm{C}$ [17]. 
In an effort to reduce detrimental effects that an electron cloud has on a beam in beam tubes, non-evaporable getter $(N E G)$ films have been developed and tested for secondary electron emission [38]. Coatings of $1 \mu \mathrm{m}$ thickness of TiZrV and TiZr have been deposited on a clean copper base by magnetron sputtering. Samples with deposited films have been exposed to air at atmospheric pressure for 24 hours before the first $S E Y$ measurement was performed. During the exposure to elevated temperatures, oxygen contained in the surface oxides diffuses into the base material, which is a process known as activation of $N E G$ films. Activation of $N E G$ films was performed in several steps at different temperatures, each maintained for 2 hours (Fig. 3.9). With the increase in activation temperature, the higher quantities of oxygen were diffused into the copper base leading to the reduction of $S E Y$ magnitude, saturating at approximately $250{ }^{\circ} \mathrm{C}$. After the exposure to the air, oxides were forming again on the $N E G$ surface. The quality of the $N E G$ surface is measured by the high oxygen solubility limit that would allow multiple activation-air exposure cycles [39].

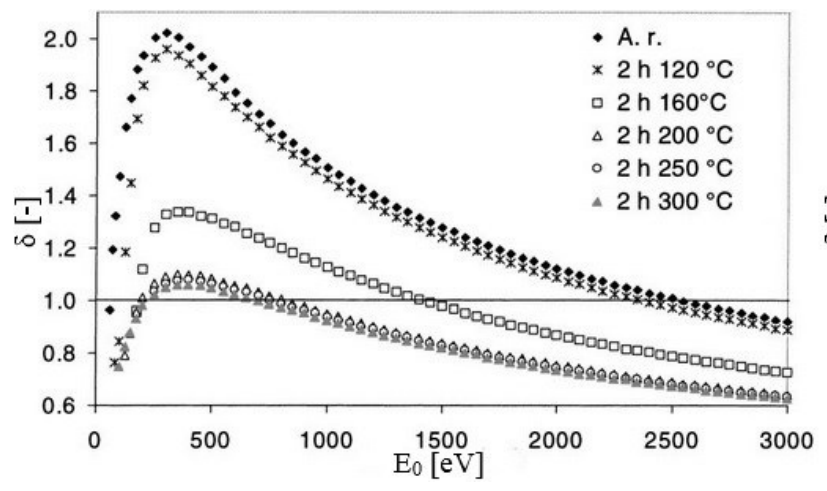

(a)

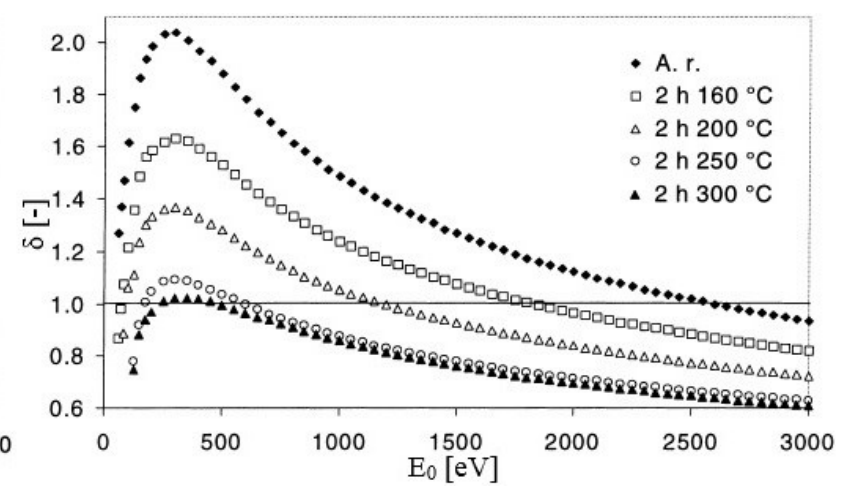

(b)

Fig. 3.9 SEY as a function of $E_{0}$, at different activation temperatures of (a) TiZrV and (b) TiZr thin films of $1 \mu \mathrm{m}$ thickness deposited on a copper base [38]. 
The results indicate that the high temperature heat treatment is an effective method to remove the evaporable gas impurities from the surface or diffuse them into the material in the case of $N E G$ coatings, consequently reducing the $S E Y$. It is important to note that all reported results were performed in a vacuum, without exposing the surface to air at atmospheric pressure. Clean surfaces have reacted with the air at atmospheric pressure forming oxides and leading to the elevated values of $S E Y[3]$.

\subsubsection{Influence of electron beam irradiation on $S E Y$}

The growth of an electron cloud formed in accelerator beam pipe is attributed mainly to synchrotron radiation [40,41]. If the number of free electrons becomes significant, it can degrade the particle beam, or cause an increase in pressure inside the beam tube. In order to mitigate the creation of an electron cloud, studies [34,42] have measured the effect of the prolonged exposure of the surface to the electron beam. For example, the copper samples were exposed to an electron beam of $500 \mathrm{eV}$ of primary energy while the beam current was in the order of 1 to $5 \mu \mathrm{A}$ [34]. The surface area of the sample exposed to the electron beam irradiation was $3 \times 3 \mathrm{~mm}$. SEY was measured on the different areas of the sample (Fig. 3.10). First, the SEY measurement was performed on the non-irradiated surface, and the maximum yield was determined to be 2.1. Second, the $S E Y$ was measured at the periphery of the irradiated area and the maximum yield measured was 1.8. Third, the central part of the irradiated surface was used to measure the $S E Y$ and the maximum value was 1.2. Differences between the periphery of the irradiated surface and the center was described as the effect of Gaussian distribution of the number of electrons across the diameter of the electron beam. Consequently, the peripheral part of the surface received less electrons than the central part of the surface. 
A similar study was performed on the aluminum sample, where a single spot of $1 \mathrm{~mm}^{2}$ was irradiated [42]. Energy of the primary electrons in the beam during irradiation was kept constant at $500 \mathrm{eV}$ while the beam current was in the order of 1 to $5 \mu \mathrm{A}$. The maximum $S E Y$ measured before the irradiation was 2.7 and after irradiation it was reduced to 1.8 . In both studies, the reduction of surface impurities and a formation of graphite layer on the irradiated surface was observed by $x$-ray photoelectron spectroscopy $(X P S)[34,42]$. The observed graphite layer was formed from the carbon present in the surface impurities.

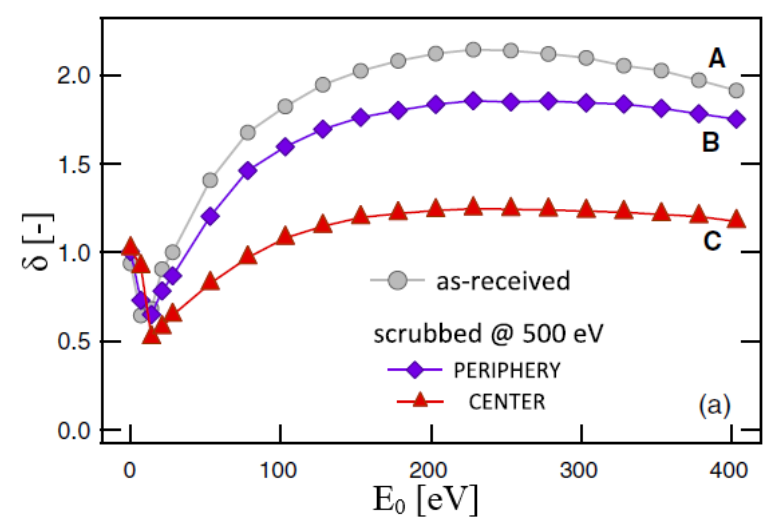

Fig. 3.10 $S E Y$ as a function of $E_{0}$, showing the effect of irradiation by an electron beam of 500 $\mathrm{eV}$ energy and $5 \mu \mathrm{A}$ of current on copper sample [34].

The experiments were performed in vacuum and no data were presented on the reactivity of the surface after exposure to air at the atmospheric pressure. The formation of the graphite layer during the electron beam irradiation is detrimental for accelerating cavity application since carbon is not a superconductor. 


\subsubsection{Influence of plasma on $S E Y$}

When superconducting technology was in the development stage for the accelerator cavities, plasma processing was considered as a method for $S E Y$ reduction [3]. Reduction of $S E Y$ was observed on niobium samples exposed to plasma of four gases (Fig. 3.11). However, due to the complex shape of the elliptical multi-cell cavities the effect of plasma on $S E Y$ was only studied on flat niobium samples. Recent developments in the technology for coaxial plasma processing made the topic current again $[43,44]$.

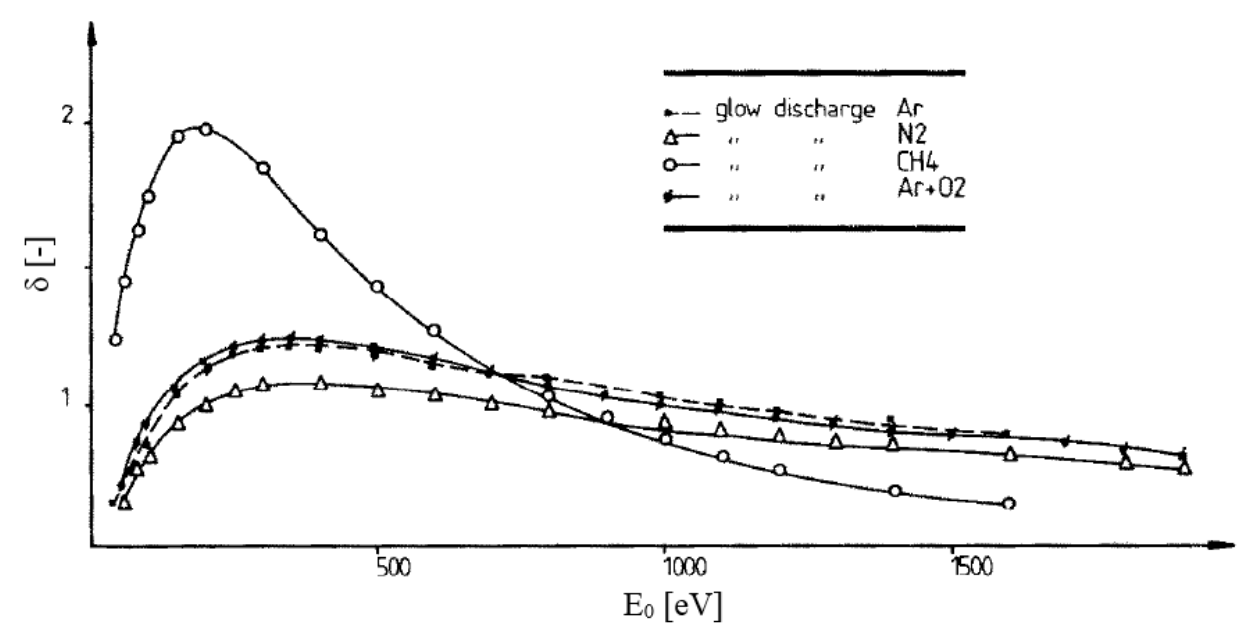

Fig. 3.11 SEY as a function of $E_{0}$, showing the effect of argon (Ar), nitrogen $\left(\mathrm{N}_{2}\right)$, methane $\left(\mathrm{CH}_{4}\right)$, and argon/oxygen $\left(\mathrm{Ar} / \mathrm{O}_{2}\right)$ glow discharge on niobium samples [3].

The effect of plasma processing on $S E Y$ of niobium samples has been tested for argon, nitrogen, methane, and a gas mixture of argon and oxygen [3]. Prior to exposing niobium samples to plasma, they were polished in a $B C P$ mixture of acids. Subsequently, the $S E Y$ curve was measured and the maximum $S E Y$ value was determined to be 2.29. After the initial $S E Y$ curve was measured, samples were exposed to plasma discharges of each of the previously 
mentioned gases. Secondary electron yield measurements were performed in-situ after plasma processing. A reduction in the maximum value of $S E Y$ was observed after plasma processing for each of the gases. The least reduction of maximum $S E Y\left(\delta_{\max }=2\right)$ was observed after processing in methane plasma. For primary electron beam energies above $1 \mathrm{keV}, \mathrm{SEY}$ of niobium surface after processing in methane plasma was reduced more than for any other gas. Processing in methane plasma could find an application where the energy levels of impacting electrons are larger than $1 \mathrm{keV}$. The lowest magnitude of maximum $S E Y\left(\delta_{\max }=1.09\right)$ was observed after processing in nitrogen plasma. Additional treatment methods were performed on the sample treated with nitrogen plasma. For example, after 94 hours in nitrogen at atmospheric pressure maximum $S E Y$ increased to 1.23 . Subsequently, the sample was heat treated at $150{ }^{\circ} \mathrm{C}$ and exposed to air at atmospheric pressure for 48 hours and the resulting maximum $S E Y$ was determined to be 1.42 .

It was observed that plasma processing is an effective method to reduce the $S E Y$ of niobium surface. However, the presented results indicate that even after the samples were kept in nitrogen gas at atmospheric pressure and heat treated, the $S E Y$ of the niobium surface increased after it was exposed to air at atmospheric pressure. It can be concluded that formulating a more efficient technique for making the inert surface after plasma processing is necessary to retain the minimal SEY values.

\subsection{Surface processing techniques of $S R F$ cavities}

The maximum accelerating gradient of $S R F$ cavities is dependent on the quality of the surface formed after preparation. Niobium cavities currently in use can attain the accelerating gradient in the range of $25-35 \mathrm{MV} / \mathrm{m}$, depending on the performed surface preparation technique. Maximum accelerating gradients in the $S R F$ niobium cavities are achieved by the combination of 
either buffered chemical polishing $(B C P)$ or electro-chemical polishing $(E C P)$, followed by the high pressure rinsing $(H P R)$ and the heat treatment in ultra-high vacuum oven. By applying these methods, average accelerating gradient of the $S R F$ cavities has been significantly improved. However, the absence of improvement in maximum accelerating field over the last few years would indicate that these surface preparation techniques might have reached their maximum potential. In order to reach the theoretical maximum accelerating gradient of niobium cavities, alternative methods of surface processing should be considered.

This section presents details of $B C P$ and $E C P$ methods, the supporting surface preparation methods of $H P R$ and heat treatment, and recently developed methods of plasma processing applied to $S R F$ cavities.

\subsubsection{Buffered chemical polishing}

A top layer of naturally forming niobium oxide $\left(\mathrm{Nb}_{2} \mathrm{O}_{5}\right)$ can be found on the surface of the cavity [45]. Additional impurities can be embedded on the surface during the fabrication procedure. After fabrication, impurities located on the inside surface of the cavity are most commonly removed by a Buffered Chemical Polishing $(B C P)$ method. A mix of hydrofluoric (HF), nitric $\left(\mathrm{HNO}_{3}\right)$ and phosphoric $\left(\mathrm{H}_{3} \mathrm{PO}_{4}\right)$ acids is used to remove the surface layers of a cavity. Surface layer removal occurs in two repetitive steps. In the first step, hydrofluoric acid is used to dissolve the $\mathrm{Nb}_{2} \mathrm{O}_{5}$, leaving behind the surface of pure niobium. During the second step, nitric acid oxidizes the pure niobium surface, building the $\mathrm{Nb}_{2} \mathrm{O}_{5}$. By repeating the oxidation and dissolution of oxide on the surface a required layer of the niobium can be removed.

The combination of $\mathrm{HF}$ and $\mathrm{HNO}_{3}$ acids has an etching rate of approximately $30 \mu \mathrm{m} / \mathrm{min}$ [46]. Acid reactions with the niobium surface are exothermic and the generated heat can cause further increase in etch rate. In addition, high quantities of hydrogen, nitrogen, and HF gases are 
produced which can be absorbed by the niobium surface leading to reduced cavity performance. To reduce the etching rate and produce a smoother surface, phosphoric acid was added to the mix. The etching rate of the $\mathrm{HF}, \mathrm{HNO}_{3}, \mathrm{H}_{3} \mathrm{PO}_{4}$ mix is around $1 \mu \mathrm{m} / \mathrm{min}$ and the average roughness of the surface after etching is in the order of $1 \mu \mathrm{m}$ (Fig. 3.12).

After $B C P$ etching, maximum achieved accelerating gradients of a nine cell cavity are in the range of $25-30 \mathrm{MV} / \mathrm{m}$ indicating the limit of the method.

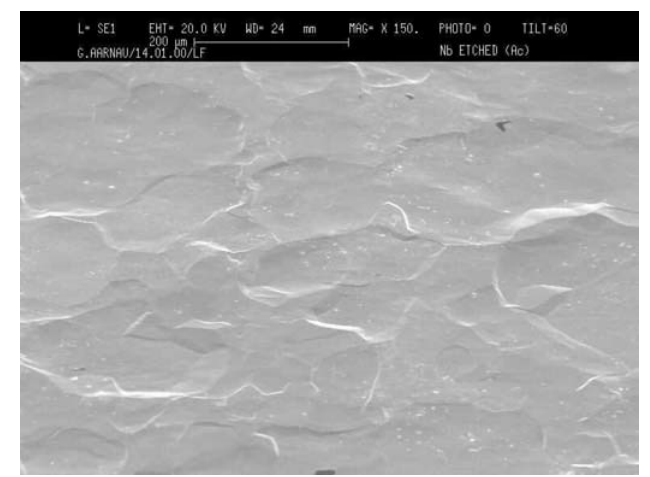

(a)

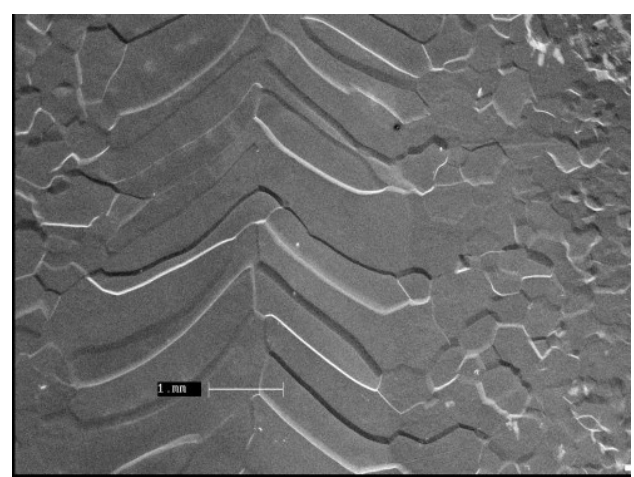

(b)

Fig. 3.12 SEM micrograph of (a) base surface of the niobium sample [46], and (b) sample with the equator weld area [47], after $B C P$ etching.

\subsubsection{Electro-chemical polishing}

The Electro-Chemical Polishing $(E C P)$ uses the combination of acid mix and current flow to remove the surface layers from niobium cavities. The most commonly used electrolyte for ECP is a mixture of hydrofluoric (HF) and sulfuric $\left(\mathrm{H}_{2} \mathrm{SO}_{4}\right)$ acids. Specifically, $\mathrm{HF}$ acid is dissolving the $\mathrm{Nb}_{2} \mathrm{O}_{5}$, while the $\mathrm{H}_{2} \mathrm{SO}_{4}$ is used to reduce the etching rate of $\mathrm{HF}$ acid. To remove additional layers from the surface, the niobium has to be re-oxidized. The oxidation of the clean niobium surface is performed by applying the small voltage (9-15 V) to the cavity [48]. The 
formation of oxide on the cavity surface can be monitored by decaying current oscillations. The oscillations represent the formation and partial reduction of the $\mathrm{Nb}_{2} \mathrm{O}_{5}$ layer. As the average oxide thickness increases, the amplitude of the current oscillations will decrease and eventually decay completely, indicating that the maximum thickness of the oxide layer was achieved. By removing the applied voltage, $\mathrm{HF}$ acid starts dissolving the oxide layer. By repeating this process a required thickness of the material can be removed.

Electro-chemical polishing produces a much smoother surface (Fig. 3.13) when compared to the $B C P$, due to the fact that the electric field is the highest on the surface protrusions which are the first to be removed [46]. After ECP surface removal, maximum accelerating gradient in the cavities was measured in the range $30-35 \mathrm{MV} / \mathrm{m}$.

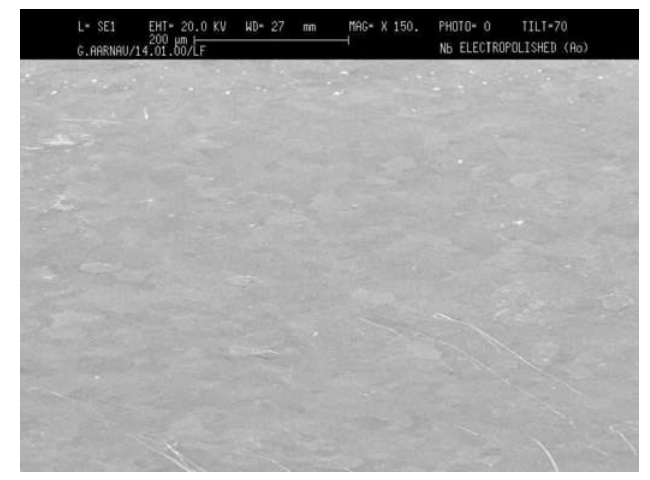

(a)

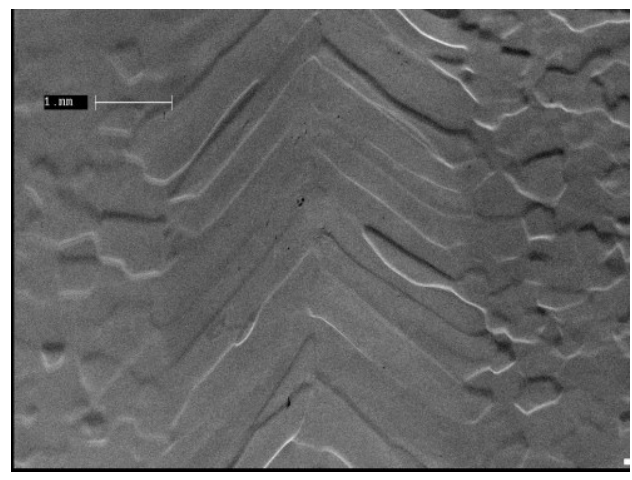

(b)

Fig. 3.13 SEM micrograph of (a) base surface of the niobium sample [46], and (b) sample with the equator weld area [47], after ECP etching.

\subsubsection{Supporting processing techniques}

To achieve the maximum accelerating gradient of the cavity, a layer thickness of approximately $100 \mu \mathrm{m}$ is typically removed by either $B C P$ or $E C P$ [4]. However, due to the 
complex shape of the multi-cell cavity, trace amounts of acids used in $B C P$ and $E C P$ may remain on the etched surface. Removal of any residuals present after etching is performed by high pressure rinsing $(H P R)$ with deionized water [49]. The water rinsing system consists of a high pressure water pump, spraying nozzle on a mechanical system that allows for scanning of the multi-cell cavity interior surface with high pressure water jets. Deionized water is circulated in a closed filtered system which prevents rinsed inclusions of getting deposited back on the surface. The $H P R$ method has proven efficient in removing trace amounts of chemical reaction products present after $B C P$ or $E C P$ etching techniques.

After electron beam welding of the half-cells, impurities like oxygen and carbon can be preferentially distributed along the weld areas of the cavity. In addition, as a result of chemical reactions during $B C P$ and $E C P$ etching, several different gases are released which can be absorbed by the processed surface and can have detrimental effect on the cavity performance. Hydrogen inclusions on the surface of the cavity may lead to the formation of niobium hydride at temperatures around $100 \mathrm{~K}$, which can cause a significant reduction of the $Q$ factor [46]. Heat treatment of the cavities in ultra-high vacuum oven at temperatures up to $1300{ }^{\circ} \mathrm{C}$ outgasses the dissolved hydrogen and homogenizes the distribution of oxygen and carbon across the surface of the cavity [49] which improves the cavity performance.

By combining either the $B C P$ or $E C P$ method with $H P R$ and heat treatment at ultra-high vacuum, maximum accelerating gradient of up to $30-35 \mathrm{MV} / \mathrm{m}$ can be achieved. The limits of the accelerating gradient are possibly due to the etching methods used. Side products of the surface etching are various gases created due to the chemical reaction between the applied acids and the surface. Even with the heat treatment in a vacuum oven with intention to evaporate gases diffused in the material, the limitation of the accelerating gradient is still present. To push 
acceleration gradient closer to the theoretical limit for niobium, alternative surface treatment methods need to be developed.

\subsubsection{Plasma processing of $S R F$ cavities}

As an alternative to $B C P$ and $E C P$ methods for $S R F$ cavity surface preparation, plasma processing has made substantial progress in both etching and cleaning techniques. Further improvement of the accelerating field magnitude that can be efficiently maintained in the accelerating cavity must be prepared by a process that removes the impurities and reduces the roughness from the inner surfaces. Recently, two research groups have reported their progress in the application of plasma processing techniques for accelerating cavities. The first group focused on developing the experimental setup that could possibly replace the $B C P$ and $E C P$ methods in surface etching of the niobium $S R F$ cavity [2]. They reported using the ionized $\mathrm{Ar} / \mathrm{Cl}_{2}$ gas mixture to remove significant quantities of niobium from ring type samples. The second group focused on reducing the field emission and multipacting of the niobium SRF cavities by employing the plasma created in $\mathrm{Ne} / \mathrm{O}_{2}$ gas mixture to improve the work function of the material surface [44]. The results from both groups are presented below.

Upadhyay et al. [2] recently developed experimental setup that employs an $r f$ capacitively coupled plasma formed in $\mathrm{Ar} / \mathrm{Cl}_{2}$ gas mixture to process the inner surface of a variable diameter cylindrical (pill-box) cavity. Plasma was formed inside the cavity which acts as outer electrode, by coaxially inserting the $r f$ powered electrode (driven electrode) and flowing the gas through the pill-box cavity. In order to create the reactive plasma capable of removing the material from the inner surface of the cavity, numerous experimental difficulties had to be overcome [50]. It was determined that the etch rate was a function of several parameters, namely positive $D C$ bias of the driven electrode, temperature of the cavity, pressure inside the cavity, $r f$ power, $\mathrm{Cl}_{2}$ 
concentration, and the inner electrode shape. The efficiency of the material removal from the surface was demonstrated on the ring type niobium samples $[2,43]$.The final design of the experimental setup included the simultaneous movement of the corrugated shape electrode and double walled conical-shape nozzle for the processing gas delivery. Simultaneous movement allowed the sectional processing of the pill-box cavity as an approach to deal with variable diameter geometry.

Compared to the current surface processing methods of $S R F$ cavities, plasma etching is a method that is more controllable, more environment friendly and less expensive [50]. Further development of the plasma processing technique may provide the means of increasing the attainable accelerating gradient of the $S R F$ cavities.

Field emission was identified as the critical problem at high gradient of an accelerating field. Reduction of the field emission can also be achieved by increasing the work function of the surface [51]. Work function represents the minimum energy required to remove an electron from a solid to vacuum. It was reported that the field emission is highly affected by the presence of any type of surface inclusions [52]. At spallation neutron source $(S N S)$, the presence of hydrocarbons released from the cavity surface was detected by a residual gas analyzer $(R G A)$ during the warm-up cycle from cryogenic temperatures [44]. The work function value was measured on the hydrocarbon covered surface was lower than on $\mathrm{Nb}_{2} \mathrm{O}_{5}$ commonly found on the cavity surface. The effect of $\mathrm{Ne} / \mathrm{O}_{2}$ plasma processing was tested as a method to improve the work function of the niobium surface by removing the hydrocarbon contaminates [53]. Reported results show a success in removing the hydrocarbon layer from surface, measured by secondary ion mass spectrometry $(S I M S)$. However, SIMS measurements repeated after 15 minutes from plasma processing showed that the hydrocarbon layer was renewed even though the sample was 
kept in vacuum. A depth analysis of the surface indicated the presence of additional hydrocarbon layers that migrated to the surface over time. To successfully remove the hydrocarbon impurities, plasma processing was repeated several times for an extended periods of time. Subsequent SIMS measurements showed a significant decrease in the hydrocarbon content over the extended period of time, and the measured work function on the niobium surface was increased. The effect of the $\mathrm{Ne} / \mathrm{O}_{2}$ plasma processing was tested on the two multi-cell cavities. One of the cavities was limited by the strong field emission to $12 \mathrm{MV} / \mathrm{m}$ which was improved to $15.8 \mathrm{MV} / \mathrm{m}$ after plasma processing. The other cavity was limited by multipacting to $9.5 \mathrm{MV} / \mathrm{m}$ and after the plasma processing maximum gradient of $21.5 \mathrm{MV} / \mathrm{m}$ was achieved. Doleans et al. [44] reported that the magnitude of multipacting might have been reduced by two mechanisms. First, the increase of the work function reduced the escape probability of the secondary electrons and consequently reduced the $S E Y$. Second, the removal of the top layers of high $S E Y$ materials may have reduced the $S E Y$ of the bulk surface.

The effectiveness of plasma in removing the surface layers of the niobium was demonstrated in [50]. Currently reported results of plasma processing [44] indicate the potential in surpassing the currently used technologies for processing the niobium $S R F$ cavities. In addition, the plasma processing technology reduces the financial and ecological cost, and offers improved process control when compared to acid bath methods. 


\section{CHAPTER 4}

\section{EXPERIMENTAL SYSTEM}

The main goal of this work was to test the effect of plasma processing on the $S E Y$ of the niobium surface. In order to perform this research, a dedicated experimental setup was designed and built to meet the requirements of the research subject. After preliminary analysis and comparison of several measurement techniques, the collector current method was selected as the $S E Y$ measurement technique, as it allowed the constant indirect control of the primary electron beam current.

This chapter is divided into four sections dealing with the following topics. The first section describes details of the developed experimental setup, the individual components, and data acquisition. In the second section, details and results of calibration measurements are given. The third section describes the details about measurement procedure and data analysis. In the end, the fourth section describes the equipment and procedure used for plasma processing of samples.

\subsection{Experimental setup for measuring $S E Y$}

The experimental setup is divided into seven components: electron gun, vacuum pumps, sample fabrication, collector fabrication, sample stage assembly, automated sample positioning system, and data acquisition (Fig. 4.1). The components and the operation of the subsystems are presented in seven sections. First, the electron gun parameters are presented in Section 4.1.1. Second, the vacuum subsystem and pump operating procedure is described in Section 4.1.2. Third, the description of sample fabrication is given in Section 4.1.3. Fourth, the description of 
the collector fabrication is presented in Section 4.1.4. Fifth, details about sample stage assembly are given in Section 4.1.5. Sixth, the description and operation of automated sample positioning system is given in Section 4.1.6. Seventh, the data acquisition system is described in Section 4.1.7. Each individual experimental component must have a stable operation at the assigned parameters to insure the quality and comparability of the results. A photograph of the experimental setup is shown in Fig. 4.2.

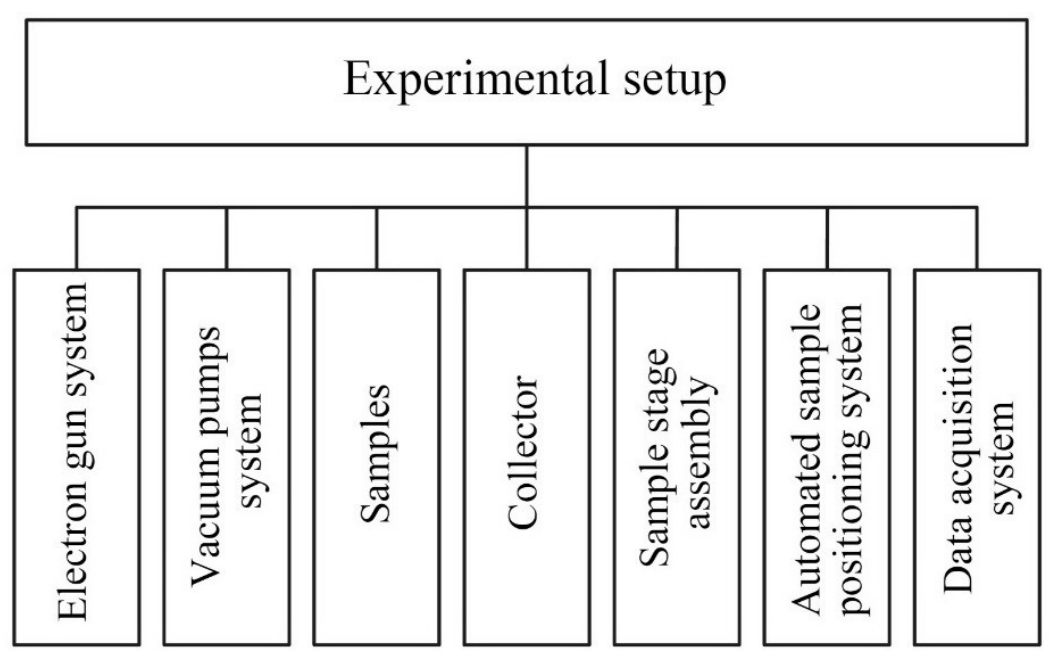

Fig. 4.1 Components of the $S E Y$ experimental setup. 


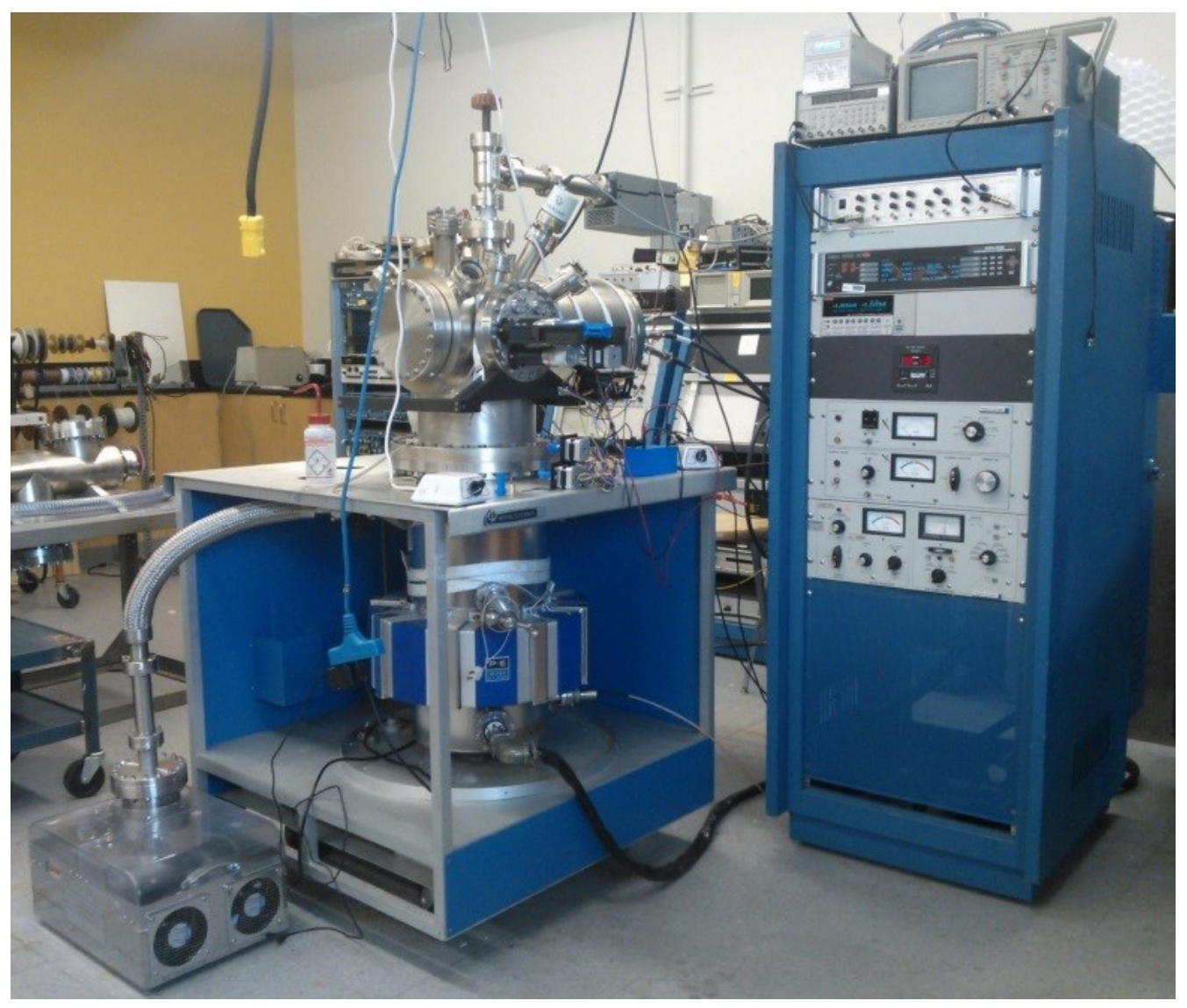

Fig. 4.2 A photograph of the experimental setup for measuring $S E Y$.

\subsubsection{Electron gun}

The source of primary electrons was chosen to be a commercial electron gun (Kimball Physics ELG-2) which was controlled and powered with the commercial power supply (Kimball Physics EGPS-1022E). The selection of the electron gun was based on its parameters of the primary electron energy range $(1-2000 \mathrm{eV})$, the primary electron beam current (up to $10 \mu \mathrm{A})$, and the size of the beam cross-section $(0.5-5 \mathrm{~mm})$. The electron gun was controlled by a computer using National Instruments PCI data acquisition cards and LabView software.

The characteristic points of the $S E Y$ curve are measured for the primary electron energies below $2000 \mathrm{eV}$ on most pure metals [29]. The energy of the primary electrons for which the $S E Y$ 
has a maximum value was reported to be in the range of $250-550 \mathrm{eV}$ for niobium $[27,29]$. The selected electron gun had a primary electron energy range large enough to contain all characteristic points of the $S E Y$ reported for a clean surface of the niobium. The energy of the primary electrons was directly controlled by adjusting the electron energy setting in the LabView software.

To avoid modifying the examined surface with the primary electron beam, the current of the beam should be as low as possible, with the recommended value below 2 nA [26]. Furthermore, the current magnitude of the electron beam was kept as uniform as possible across the energy range of the primary electrons to maintain the conditions of the $S E Y$ measurements. Primary electrons are released from the surface of the tantalum filament by applying potential difference across it. By adjusting the filament voltage in LabView software, the filament current was controlled. Due to the effect of Joule heating, electrons are released from the surface of the filament, which are used to form the electron beam. The final magnitude of the beam current was adjusted by controlling the voltages on a set of electro-optics in the electron gun. Once the filament starts emitting free electrons, fine tuning of beam current magnitude was adjusted by controlling the Grid and $1^{\text {st }}$ Anode voltages in the LabView software. Grid voltage caused the reduction in the number of electrons leaving the surface of the filament, while the $1^{\text {st }}$ Anode voltage increased the number of emitted free electrons. By combining the magnitudes of Filament, Grid, and $1^{\text {st }}$ Anode voltage, a primary electron beam current up $10 \mu \mathrm{A}$ can be achieved.

Primary electron beam size was controlled by adjusting the Focus voltage setting in the Labview software. However, the Focus voltage values required to maintain the cross-section size of the electron beam for the entire energy range was also a function of Filament, Grid, and $1^{\text {st }}$ 
Anode voltages. The required Focus voltage level needed to maintain the electron beam crosssection at different energies of the primary electrons was determined for constant values of Filament, Grid, and $1^{\text {st }}$ Anode voltages.

Based on the experimental requirements of energy levels, beam current magnitudes, and size of the electron beam cross-section, the selection of the electron gun and the power supply was made.

\subsubsection{Vacuum system}

Secondary electron yield measurements can only be performed at high vacuum levels and the developed experimental setup was able to achieve the pressure of $2 \cdot 10^{-9}$ Torr. The manufacturer recommended minimum operating vacuum level of the electron gun is defined as $10^{-7}$ Torr or better, to avoid damaging the filament. High levels of vacuum reduce energy dissipation and beam divergence of the primary electrons. To achieve the required level of vacuum, a pumping system of three pumps and two vacuum gauges was implemented. First, the scroll pump (Agilent Varian IDP-3) was used to evacuate the system starting from atmospheric pressure to $10^{-1}$ Torr. Second, the turbo-molecular pump (Agilent Turbo-V 301 Navigator) reduced the pressure down to $10^{-7}$ Torr. Third, the ion pump (Perkin Elmer TNB-X) was used to achieve pressure below $10^{-8}$ Torr and maintain the vacuum level during the experimental procedure. The first vacuum gauge (Varian FRG-700) measured the pressure levels from atmospheric to $10^{-8}$ Torr. The second vacuum gauge (KJLC 423 Series) was used to measure the pressure levels from $10^{-2}$ Torr to $10^{-11}$ Torr. Vacuum system was repeatedly able to achieve the base pressure of $2 \cdot 10^{-9}$ Torr, which was sufficient to perform SEY measurements. During the electron gun operation, pressure in the vacuum chamber did not rise above $4 \cdot 10^{-9}$ Torr. 
The three pumps were used to compensate for the limitations of each individual pump. The required vacuum level could only be achieved by a combined operation of all pumps. For example, the scroll pump can only achieve the pressure of $10^{-1}$ Torr. On the other hand, the turbo-molecular pump can operate efficiently only with the support of the scroll pump. For that reason, these two pumps have been connected in a series and were then linked with the vacuum chamber. The ion pump can operate after the vacuum level of at least $10^{-6}$ Torr was achieved.

In order to attain and keep as low a pressure level as possible, all surfaces inside the vacuum system must be clean. Impurities present inside the vacuum chamber will begin to evaporate at reduced pressure, limiting the maximum achievable vacuum. For example, water and oil require a significant amount of time to evaporate, thus extending the pump-down time. For that reason, all surfaces and components exposed to the vacuum were cleaned with ethanol and handled carefully with nitrile gloves to avoid contaminating the surface. Ethanol was used for cleaning since it is a fast evaporating solvent.

Once the vacuum chamber was closed, the scroll pump was started and the maximum vacuum level of $10^{-1}$ Torr was achieved. At that moment, the turbo-molecular pump started which caused a further pressure decrease to high $10^{-6}$ Torr range. It took approximately 24 hours to reach this vacuum level. The pressure stabilization at this level was caused by the evaporation of water molecules absorbed by the surfaces from air humidity during exposure to atmosphere. To expedite the evaporation, vacuum chamber temperature was increased by wrapping it with heating tapes. The vacuum chamber was baked for 48 hours at $175^{\circ} \mathrm{C}$, which was half of the highest baking temperature of the electron gun recommended by the manufacturer. Evaporated water molecules left the surface and were removed from the system by turbo-molecular and scroll pump. After the first 24 hours, the pressure in the vacuum chamber stabilized in the $10^{-7}$ 
Torr range and the ion pump was prepared for operation. This was performed by starting and stopping (chopping) the ion pump several times, for about two seconds. After each chop, the gas trapped in the electrode surface was released and removed from the vacuum chamber by turbomolecular and scroll pumps. Chopping was performed until there was no significant increase in pressure measured by the vacuum gauges. Subsequently, the ion pump was started, and the valve separating the turbo-molecular and scroll pumps from the vacuum chamber was closed. After 24 hours of ion pump operation, the pressure in the vacuum chamber was stabilized in the high $10^{-8}$ Torr range, and the baking was stopped. With the cooldown, the inside surface of the vacuum chamber absorbed part of the remaining air molecules, reducing the pressure to the base value of $2 \cdot 10^{-9}$ Torr.

The operating procedure of the described vacuum system was repeatedly achieving the base pressure, which was sufficient for continuous operation of the electron gun during the $S E Y$ measurements. The increase in pressure measured during the electron gun operation never exceeded $4 \cdot 10^{-9}$ Torr.

\subsubsection{Sample fabrication}

Three sets of samples were fabricated from the cavity grade niobium to include the characteristic microstructures present on the accelerating $S R F$ cavity surface. The niobium sheet metal plate was cut into strips that were welded by the electron beam to replicate the weld areas of equator and iris edges of the cavity. Extraction of the samples from the plate was performed using the waterjet cutting machine and in such position to include a weld zone (WZ), a heat affected zone (HAZ) and a base niobium (BASE). Samples were made to be circular with diameter of $20 \mathrm{~mm}$ and the thickness of $3 \mathrm{~mm}$. A mounting hole with an $\mathrm{M} 2$ thread was made on the back surface of the samples. A total of ten samples were fabricated for each set. Using $B C P$ 
method, a $5 \mu \mathrm{m}$ layer was etched from the surface of nine samples to remove any impurities embedded during the waterjet or mounting hole threading procedure. The remaining sample did not go through any surface preparations.

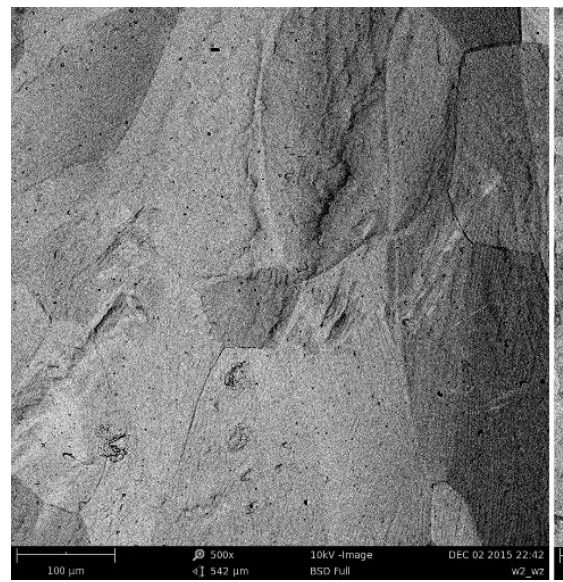

(a)

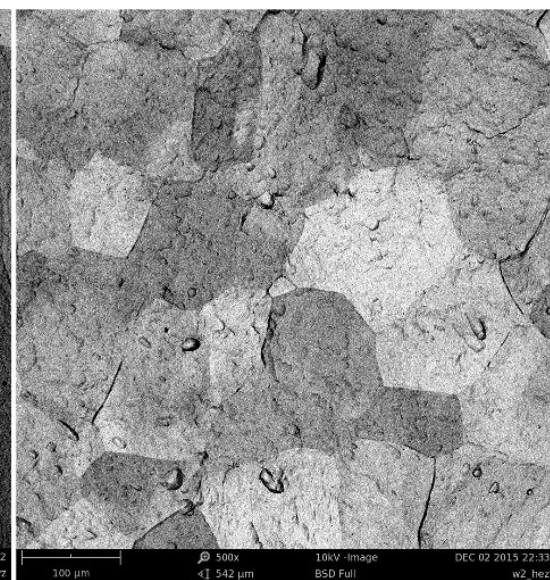

(b)

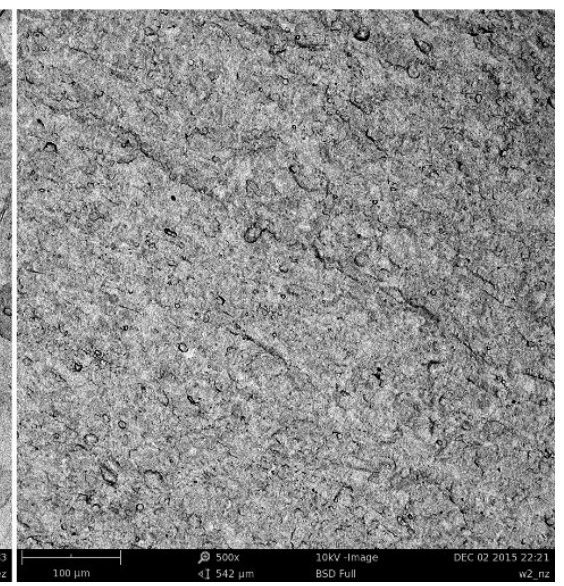

(c)

Fig. 4.3 The SEM pictures of three different microstructures of characteristic surfaces taken at x500 magnification scale. Surfaces correspond to the (a) WZ, (HAZ), and (c) BASE.

The $S R F$ cavities have areas of different microstructures on its surface as a consequence of the fabricating procedure. Differences in the microstructure are formed during the electron beam welding process. Three different surface microstructures can be observed in equator and iris weld areas. First, the weld zone (WZ) forms where the niobium was melted to join the two half cells during welding (Fig. 4.3 (a)). Second, the heat affected zone (HAZ) was formed in the area around the weld bead due to the effect of heat dissipation through the material (Fig. 4.3 (b)). A high temperature causes the increase in size of the microstructure grains in $H A Z$. Third, the initial microstructure (BASE) of the niobium was found at the distance from the weld zone where the heat energy level was low enough not to cause any microstructure change (Fig. 4.3 (c)). The 
$B A S E$ surface was divided into a high and a low temperature section, where the high temperature section was adjacent to the $H A Z$ and the low temperature section which was farther from the $H A Z$. Separation of surfaces was made due to the reported influence of high temperature on the $S E Y$.

Table 4.1 List of impurities in niobium sheet metal, sorted by the total content (maximum percent). Chemical content was determined by the Eagle Alloys Corporation.

\begin{tabular}{cccc}
\hline \multicolumn{4}{c}{ Chemical content (maximum percentage) } \\
\hline Element & Content [\%] & Element & Content [\%] \\
\hline $\mathrm{Ta}$ & 0.1 & $\mathrm{~N}$ & 0.004 \\
$\mathrm{Mo}$ & 0.009 & $\mathrm{Ni}$ & 0.002 \\
$\mathrm{Zr}$ & 0.008 & $\mathrm{Si}$ & 0.002 \\
$\mathrm{Hf}$ & 0.007 & $\mathrm{~W}$ & 0.001 \\
$\mathrm{Fe}$ & 0.006 & $\mathrm{O}$ & 0.0012 \\
$\mathrm{C}$ & 0.005 & $\mathrm{H}$ & 0.0007 \\
\hline
\end{tabular}

In order to measure $S E Y$ of the characteristic surfaces present on the surface of the accelerating cavity, three sets of samples were fabricated from the cavity grade niobium. The niobium sheet metal used for sample fabrication was obtained from Eagle Alloys Corporation. The chemical composition of the niobium sheet metal was determined by the vendor and it is presented in Table 4.1. Samples were fabricated by cutting the sheet metal into three strips and then welding them together by the electron beam with the same welding parameters used for cavity fabrication. After setting the plates in the welding position, the chamber has been evacuated to $5 \cdot 10^{-5}$ Torr. An initial preheat pass of $16 \mathrm{~mA}$, and $50 \mathrm{keV}$ electron beam with the plate speed of 20 inches per minute was made. Subsequently, a beam of $42 \mathrm{~mA}, 50 \mathrm{keV}$ with 18 inches per minute was used to create a full penetration weld and join the plates. For both passes, the electron beam was rastered in an oval shape with 0.275 inches along the weld direction and 
0.080 inches transverse to the weld direction. Rastering was performed to create a stirring effect in the molten metal and allow the escape of trapped gasses. After welding, the plate remained in the vacuum of the welding chamber for a cooldown. Once the welded plate reached ambient temperature, it was removed from the vacuum chamber for sample extraction. The sample extraction was performed by a waterjet cutting machine to avoid inducing additional heat into the niobium samples. In addition, the mounting hole with the M2 thread was made at the back of the sample which was used to attach the sample to sample stage (Fig. 4.4).
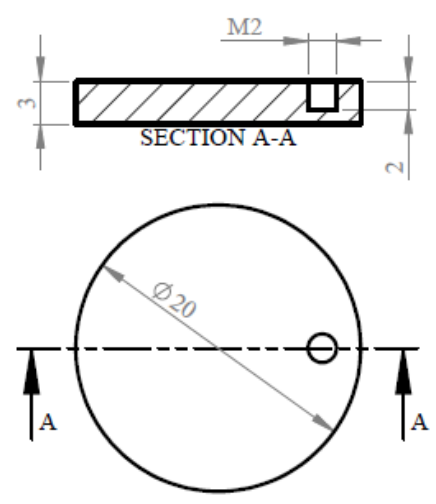

Fig. 4.4 Sketch of the sample with a sectional view showing mounting hole.

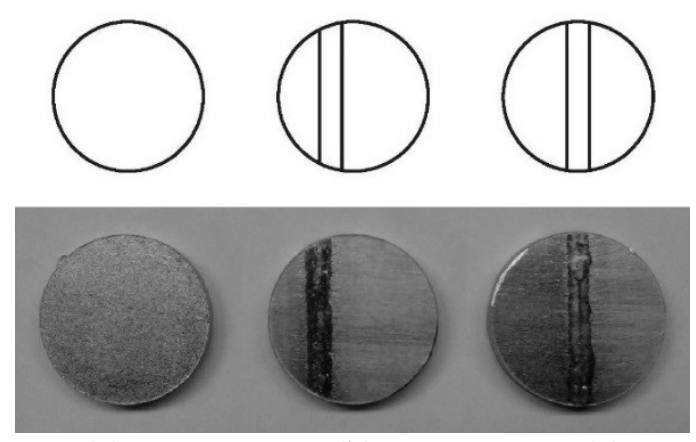

(a) (b)

(c)

Fig. 4.5 Sketch of the samples from each set (top row) and their matching photographs (bottom row). Samples are from sets as follows: (a) BASE set, (b) OFFSET set, and (c) WELD set. 
Three sample sets are extracted to include the characteristic surfaces present on the cavity after fabrication procedure (Fig. 4.5). The first sample set is named $B A S E(B)$ and it represents the surface of the cavity that is found half way between the iris and equator weld. A characteristic of this sample set is the uniformity of microstructure. The second set is named OFFSET $(O)$ describing the position of the sample taken from weld bead with respect to the center line of the sample. The samples from this set have characteristic surfaces of $W Z, H A Z$, and the high temperature section of the base metal adjacent to the heat affected zone $(R-H A Z)$. The third sample set is named WELD, and it contains the $H A Z$ and $W Z$ characteristic surfaces. In the WELD sample set, the weld bead is located across the middle of the sample surface.

There were a total of ten samples fabricated in each set, one for every incident angle of primary electrons plus one additional sample. Nine samples from each set underwent $B C P$ etching to remove any impurities from the surface absorbed during waterjet cutting and the making of the mounting holes. A sample from each set was excluded from $B C P$ to test the $S E Y$ of the niobium without any additional surface preparations after welding.

\subsubsection{Collector fabrication}

A custom collector of the electrons emitted from the surface of the sample was designed to be mounted on the mouth of the electron gun. The designed collector allowed the transition of the primary electron beam to the surface of the sample and collection of the emitted electrons. Furthermore, the collector had to be large enough to permit the measurement of SEY at multiple spots on the sample at different incident angles of the primary electrons. These requirements placed the constraints on the dimensions of the collector and the maximum incident angle of primary electrons for which all emitted electrons can be collected. The adopted collector design was fabricated out of titanium due to its low SEY magnitude [27]. 


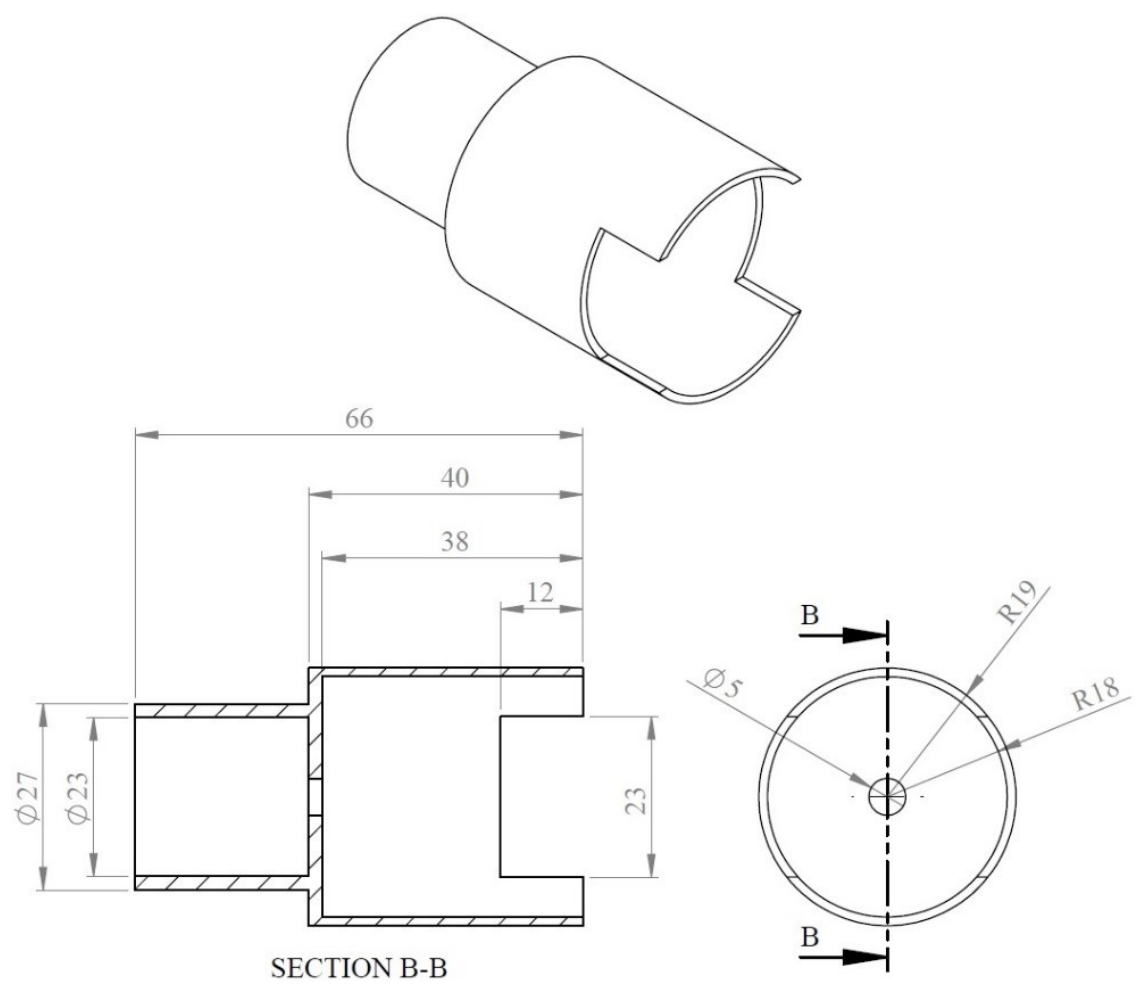

Fig. 4.6 Sketch of the collector design (top) and cross-section of the collector with dimensions (bottom).

The collector was designed to be mounted on the mouth of the electron gun (Fig. 4.6). To electrically separate the surfaces of the collector and the electron gun, a Teflon cap was fabricated that was fitted on the electron gun before the collector. To allow the transition of the primary electrons from the electron gun to the sample surface, a $5 \mathrm{~mm}$ aperture was made on the collector. The inner diameter of the collector was chosen to allow nine measurements spots on the sample surface at normal incident angle of primary electrons. The number of measurements was limited to avoid the contact between the surfaces of the sample and the collector which would create a short circuit in the current measurement. Brims were added to the top and the bottom of the collector to extend the range of incident angles of primary electrons for which all emitted electrons can be collected. The arc length of the brims was limited to allow the rotation 
of the sample stage and avoid the contact of the sample with the collector. The length of the brims was also limited to avoid contact of the collector with the components of the sample stage at different measuring spots and incident angles of samples. The final design of the collector allowed gathering of all emitted electrons in the range of $\pm 60^{\circ}$ incident angle of the primary electrons. To avoid contact between the surface of the collector and the sample stage, the number of measuring spots on the sample surface for incident angles of $\pm 45^{\circ}$ and above was reduced.

The fabricated collector allowed the transition of the primary electrons through the aperture without affecting the direction and the current magnitude of the electron beam. Electrons emitted from the surface of the sample were completely collected in the range of $\pm 60^{\circ}$ incident angle of the primary electrons.

\subsubsection{Sample stage assembly}

The large number of measurements that needed to be performed required a sample manipulation system in order to increase test efficiency. Therefore, it was necessary to create a system that will satisfy the requirements of multiple measuring spots on a single sample, mounting multiple samples, and for a range of incident angles of primary electrons at which the SEY can be measured. To that end, the apparatus was assembled from the combination of commercial and custom parts. The foundation of the system was a sample stage (PHI 15-610 Physical Electronics) that could carry up to twelve samples. The sample stage had four degrees of freedom, three translational axes forming a 3D Cartesian coordinate system for sample positioning, and one rotational motion that allowed changing the sample under examination while in the vacuum (Fig. 4.7). Specifically, each axis of translational motion allowed $\pm 12.5 \mathrm{~mm}$ movement with $2 \mu \mathrm{m}$ increment, whereas the rotation had 12 preset positions, one for each sample that the stage could carry. In addition, the sample stage was designed for high vacuum 
environments and had four vacuum $B N C$ feedthroughs. Available $B N C$ connectors have been used to connect the current measuring equipment and the power supply used for sample biasing.

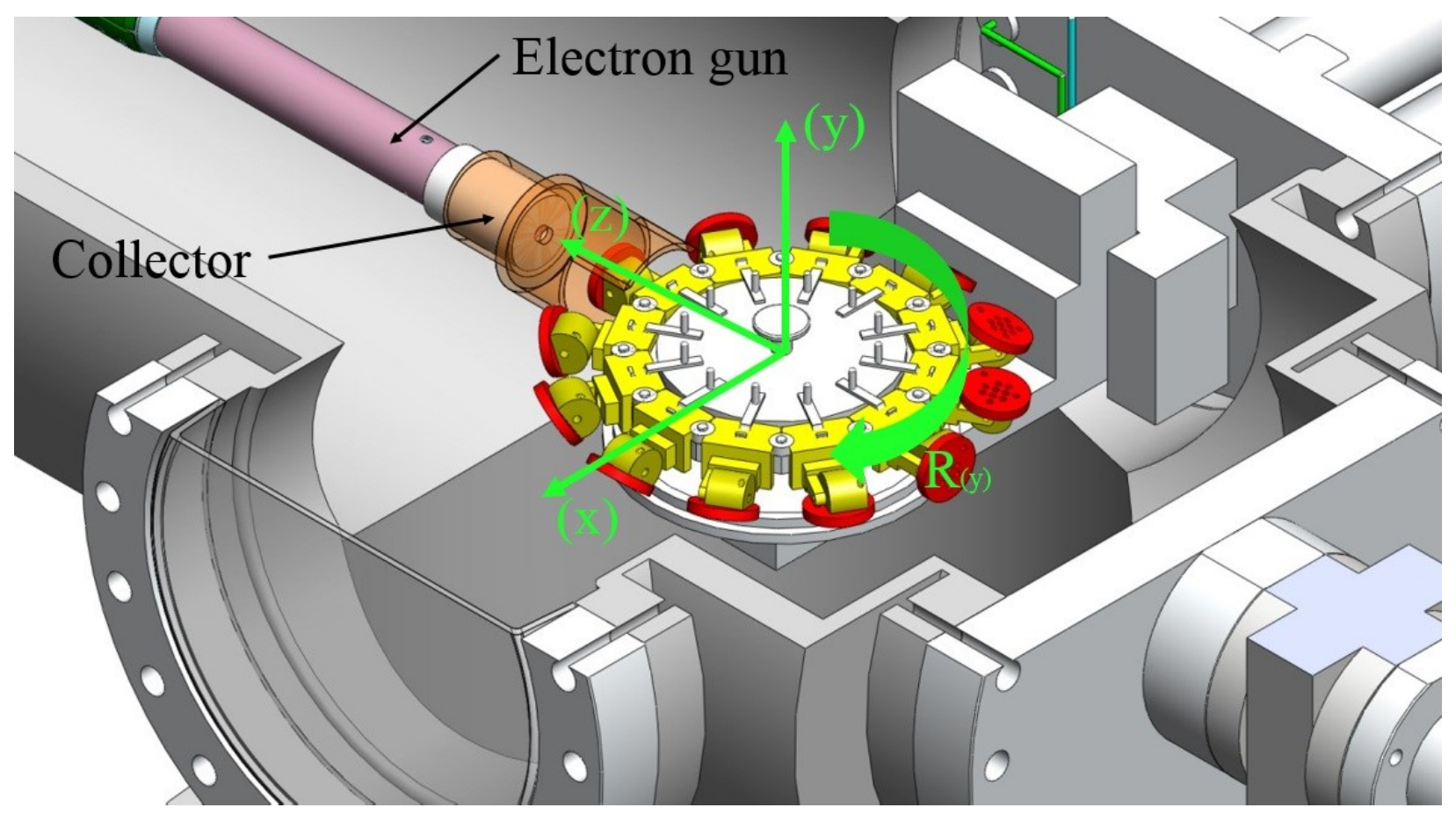

Fig. 4.7 3D model of the experimental setup interior showing the directions of translational ((x), $(\mathrm{y})$, and $(\mathrm{z})$ ) and rotational motions $\left(\mathrm{R}_{(\mathrm{y})}\right)$, and location of the electron gun with the mounted collector [54].

The sample stage provided the means of mounting multiple samples at one time, however the challenge of mounting the samples at specific angles still needed to be addressed. In addition, the sample mounts had to be compatible with high vacuum and electrically conductive. A large number of different sample mounts have been developed for application in various surface characterization equipment. However, very few holders were compatible with all presented requirements. At the end, a sample holder with a rotatable mount base (PELCO SEMClip 1533910) was selected to carry the samples and tilt them at specific angles. Additional modifications 
were made to the sample holder to accommodate the sample size and increase the tilt angle of the sample.



Fig. 4.8 Cross-section of the electron gun and the collector assembly, with a centered sample at normal incident angle.

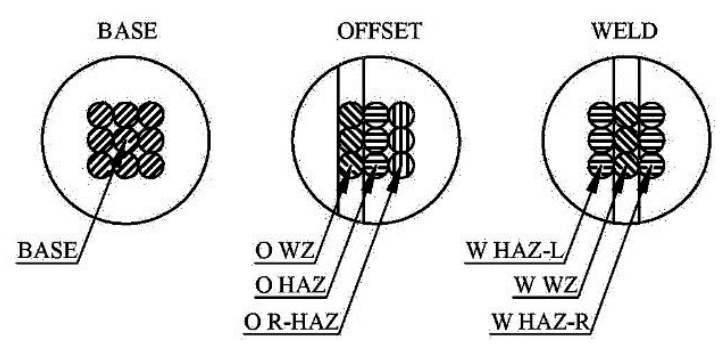

Fig. 4.9 Sketch of the samples from each set with the location of the 9 measurement spots, at normal incident angle.

Once the samples were mounted on the sampler holders, the assembly was attached to the sample stage. The sample was positioned in front of the electron gun and aligned with the collector axis (Fig. 4.8). With the sample positioned, three micrometer calipers on the sample stage were set to $12.5 \mathrm{~mm}$ mark which was the middle value of the total distance that sample stage can travel in one direction. Once the position of the sample center was recorded, the 
placements of the additional measuring spots could be determined. For a sample surface perpendicular to the primary electron beam $\left(0^{\circ}\right)$, a total of nine testing locations were determined in a 3 by 3 pattern (Fig. 4.9). Fig. 4.9 also indicates the number of measurements that can be performed at a characteristic surface of three sample sets. The distance between the spot centers was $3 \mathrm{~mm}$ in each direction of the primary axes to avoid the overlapping of the surfaces irradiated with the electron beam and stay within the characteristic surface on the sample. Each measuring spot has a set of three coordinates that describe its position relative to the centered sample. Since one of the goals was to determine the $S E Y$ as a function of the incident angle of primary electrons, as a set of coordinates for each measuring spot was determined at each observed angle. Specifically, the sample was rotated on the holder to form an incident angle with primary electrons in a range of $\pm 60^{\circ}$ with $15^{\circ}$ increment (Fig. 4.10). The rotation axis of the sample holder was located behind the examined surface (Fig. 4.8). Therefore, each measuring spot at a specific angle required a correction in the set of coordinates to keep the spot position constant with respect to the electron beam.

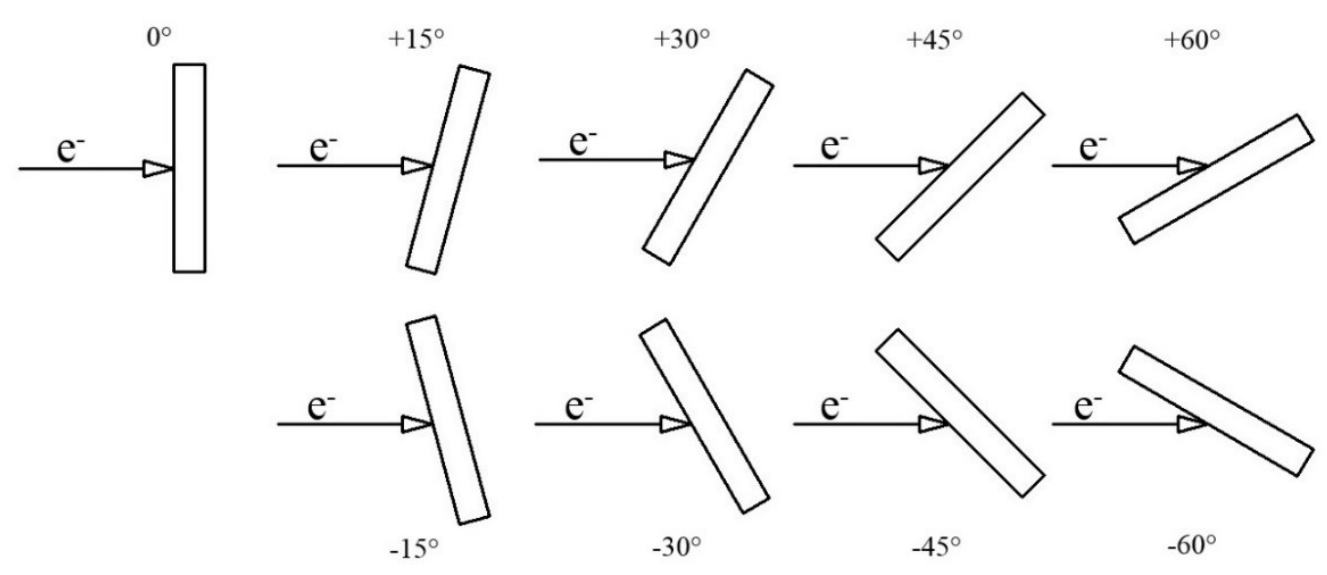

Fig. 4.10 Sketch of the incident angles $(\theta)$ that were formed by the normal of the sample surface and the primary electron beam. 
By including the adjustments of the sample position, all experimental parameters except incident angles were kept invariant. Consequently, any observed change in the $S E Y$, on a uniform surface, should only be a function of the angle variation. However, with the increase in the absolute angle of inclination, positioning correction values became large enough to cause the contact between the collector and the sample holder. To avoid the contact between the sample and the collector, the number of measurement spots was reduced for incident angles above $\pm 45^{\circ}$. For instance, it was possible to measure 9 different spots on the samples in the range of $\pm 30^{\circ}$, but for angles at $\pm 45^{\circ}$ and $\pm 60^{\circ}$, only 6 and 3 measuring sites were possible, respectively. The number of measuring spots available and surface viewed from the primary electron beam direction at different incident angles was shown in Fig. 4.11. To assure correct positioning, relative sets of coordinates with included corrections for testing sites have been recalculated to absolute sets of coordinates. Absolute sample stage coordinates for the central spot on the surface are given in Table 4.2.
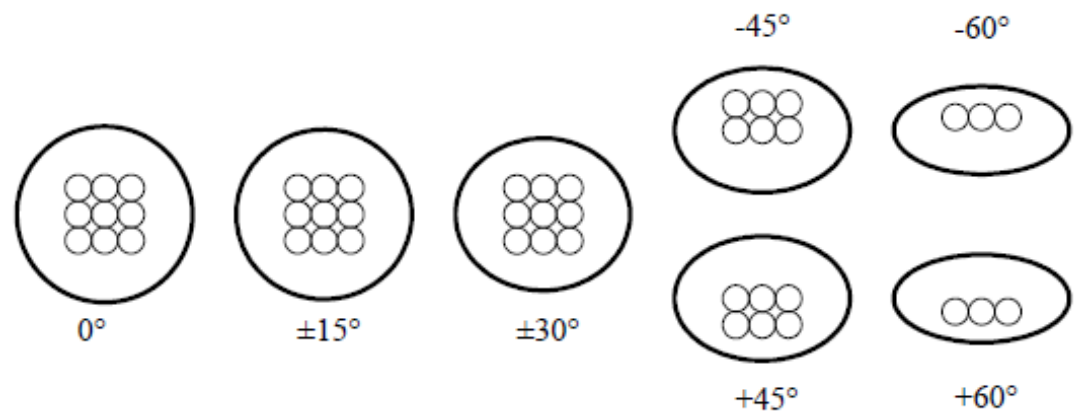

Fig. 4.11 Surface of the sample observed from the primary electron beam direction at different angle of sample inclinations and the number of measuring spots with locations for each angle. 
Table 4.2 Absolute sample stage coordinates for the middle row/center column spot on the surface at all incident angles. Distance from the electron gun mouth was denoted by (z) axis, vertical position was denoted by (y) axis, and horizontal position was denoted by (x) axis.

\begin{tabular}{cccccccc}
\hline \hline Sample & \multicolumn{3}{c}{ Coordinates $[\mathrm{mm}]$} & & Sample & \multicolumn{3}{c}{ Coordinates [mm] } \\
\hline Angle & $(\mathrm{z})$ & $(\mathrm{y})$ & $(\mathrm{x})$ & Angle & $(\mathrm{z})$ & $(\mathrm{y})$ & $(\mathrm{x})$ \\
$0^{\circ}$ & 19.00 & 12.5 & 12.50 & $0^{\circ}$ & - & - & - \\
$+15^{\circ}$ & 19.23 & 10.72 & 12.50 & $-15^{\circ}$ & 19.23 & 14.28 & 12.50 \\
$+30^{\circ}$ & 19.92 & 9.07 & 12.50 & $-30^{\circ}$ & 19.92 & 14.28 & 12.50 \\
$+45^{\circ}$ & 21.00 & 7.65 & 12.50 & $-45^{\circ}$ & 21.00 & 17.35 & 12.50 \\
$+60^{\circ}$ & - & - & - & $-60^{\circ}$ & - & - & - \\
\hline
\end{tabular}

\subsubsection{Automated sample positioning system}

An automated system was developed for sample manipulation in order to reduce the time of positioning the samples at different measuring spots. The number of measuring spots at all angles together with the set of three coordinates leads to a long time required to position the samples manually. To avoid manual positioning of the samples, a custom automated system was designed that was able to store all positions of measurement spots at all angles of inclination, and position the individual sample appropriately for a recalled measurement spot.

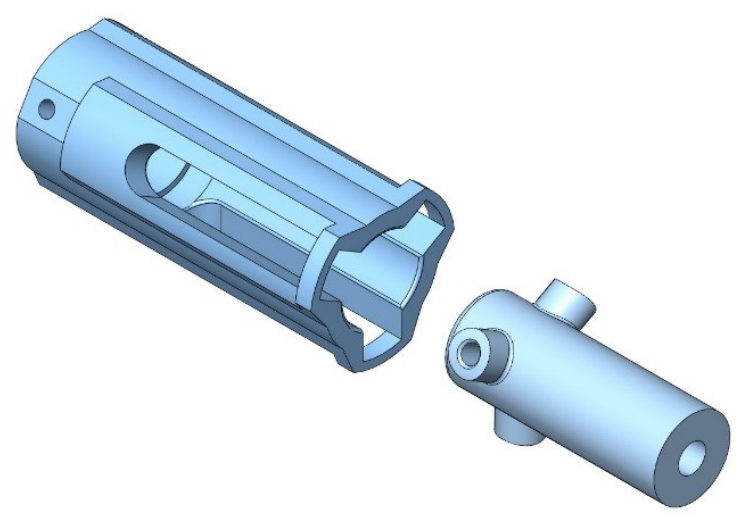

Fig. 4.12 The interface mechanism used to transfer the rotational motion of the stepper motors to micrometer calipers of translational motion, while allowing free axial movement. 
The automated positioning system contained several components. First, each degree of freedom was powered by a stepper motor. Translational motion axes were powered by stepper motors (Wantai SM-45BYG011) which provided $2.5 \mu \mathrm{m}$ of linear motion per single step of the motor. Since the accuracy of the micrometer calipers on the sample stage were $2 \mu \mathrm{m}$, the least common multiple of 2.5 and 2 was $10 \mu \mathrm{m}$, which was the step size of the sample positioning. For the rotation of the sample stage, a stepper motor (Wantai SM-42BYGHM809) with higher torque was required. Stepper motors were controlled by Arduino Mega 2560 and the touch screen display with a custom graphics user interface $(G U I)$. Coordinates of the measurement spots were recorded in the GUI software which were recalled for the $S E Y$ measurement, positioning the selected spot of the sample in front of the electron beam. Second, a mechanism interface was designed to transfer the rotational motion of the stepper motors to the sample stage, while allowing the free translational movement of the micrometer calipers (Fig. 4.12). In addition, to avoid the accumulation of error in positioning during the extended period of operation, stiffness of the mechanism was crucial. At the end, the adopted design was fabricated in a 3D printer (MakerBot Replicator) and tested. The results of mechanism interface testing confirmed the negligible accumulation of positioning error over multiple trials. The housing case for the electronics and the stepper motor mounts were designed to be attached to the sample stage. Photos of the assembled automated system are shown in Fig. 4.13. 


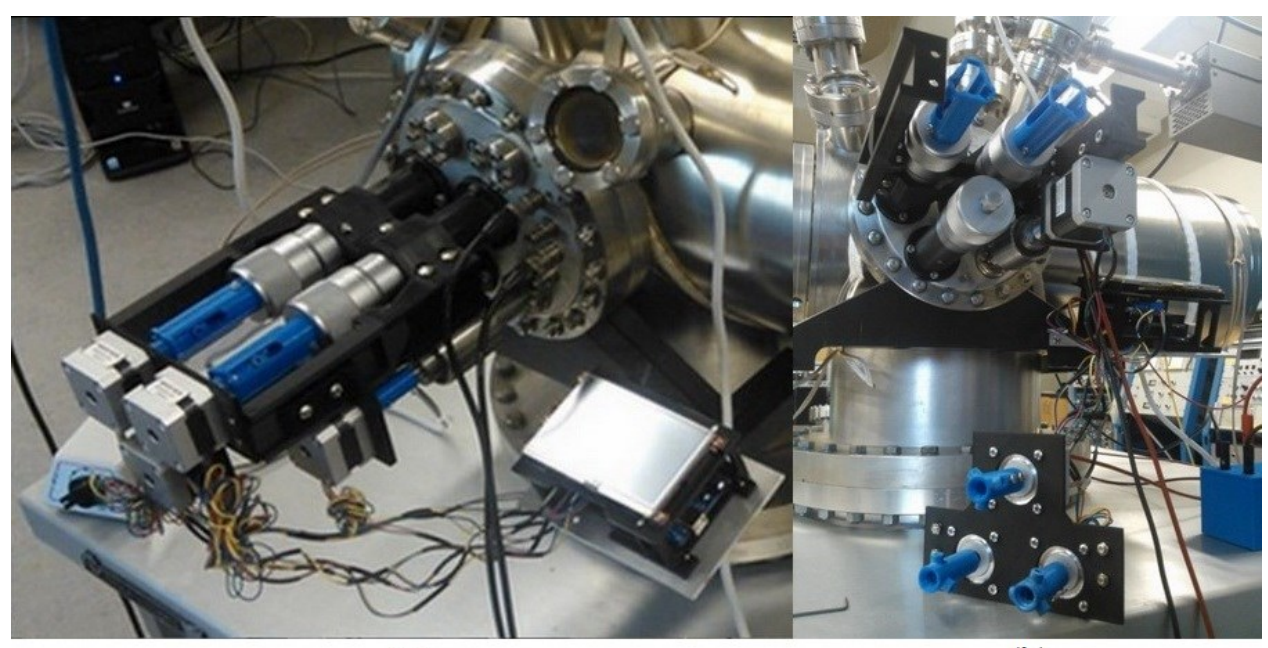

(a)

(b)

Fig. 4.13 (a) Assembled automated system for sample positioning, and (b) stepper motors removed from the sample stage.

\subsubsection{Data acquisition system}

An experimental setup was developed to measure $S E Y$ by utilizing the collector current method. Synchronized measurement of the collector and sample currents was necessary to accurately determine the $S E Y$ magnitude. A dual channel picoammeter (Keithley 6482) was employed to measure the collector and the sample currents simultaneously and store the data to an integrated memory buffer. Measurement data were later transferred to $P C$ for further analysis. To obtain accurate measurements, low noise coaxial cables were used to connect the picoammeter with the collector and the sample. Furthermore, voltage sources were added to the electric circuit which allowed biasing of the sample and the collector and controlling the flow of emitted electrons between their surfaces. Multiple impacts of emitted electrons were prevented by positively biasing the collector and negatively biasing the sample.

By using the collector current method to determine the $S E Y$, the current had to be measured simultaneously on the collector and the sample. The current measurement was performed with a 
dual channel picoammeter. During measurements, current magnitudes of the collector and the sample were recorded in the memory buffer of the picoammeter. The collector current was measured by connecting a low noise coaxial cable between the picoammeter and the $B N C$ connector located on one of the flanges of the vacuum chamber. Inside the vacuum chamber, a connection between the collector and the $B N C$ connector was made by a ceramic shielded copper wire. A ceramic insulation was used to prevent the short circuit that would occur during contact between the copper wire and the vacuum chamber wall. The sample current was measured by connecting a low noise coaxial cable from the picoammeter to one of the $B N C$ feedthroughs on the sample stage. Inside the vacuum chamber, the sample stage already had a connection between the sample and the $B N C$ feedthrough. Electric current noise levels from the cables and connections measured on the picoammeter were $\pm 1 \mathrm{pA}$ on both the collector and the sample measurement channel. Increased current noise levels (around $\pm 7 \mathrm{pA}$ ) were observed on both channels as a consequence of floor vibrations generated during movement in the proximity of the experimental setup. In order to keep the current noise levels as low as possible, electron gun control and data acquisition were performed remotely by using a $P C$. In addition, access to the experimental setup was restricted during the measurement of the $S E Y$ to avoid raising the current noise level due to vibrations. During the remote measurement of the $S E Y$, current noise levels were in the range $\pm 1 \mathrm{pA}$. With the primary electron beam current always above $300 \mathrm{pA}$, error in measurements due to current noise level was always less than $1 \%$.

The collector and sample current were measured simultaneously while the primary electron beam was directed towards the sample surface. Due to the secondary electron emission property of the material, electrons were emitted and reflected from the sample surface back to the collector surface at various angles. By measuring the collector and sample currents, the $S E Y$ can 
be calculated using Eq. (3.2). The sketch of the measurement circuit was presented in Fig. 4.14. To ensure that electrons leaving the sample surface were not returning, a negative bias was applied to repel them. On the other hand, the collector was biased to a positive voltage to retain all incoming electrons and prevent the formation of the tertiary electrons. Biasing of the sample and collector was necessary to prevent the transfer of electrons from collector to the sample surface.

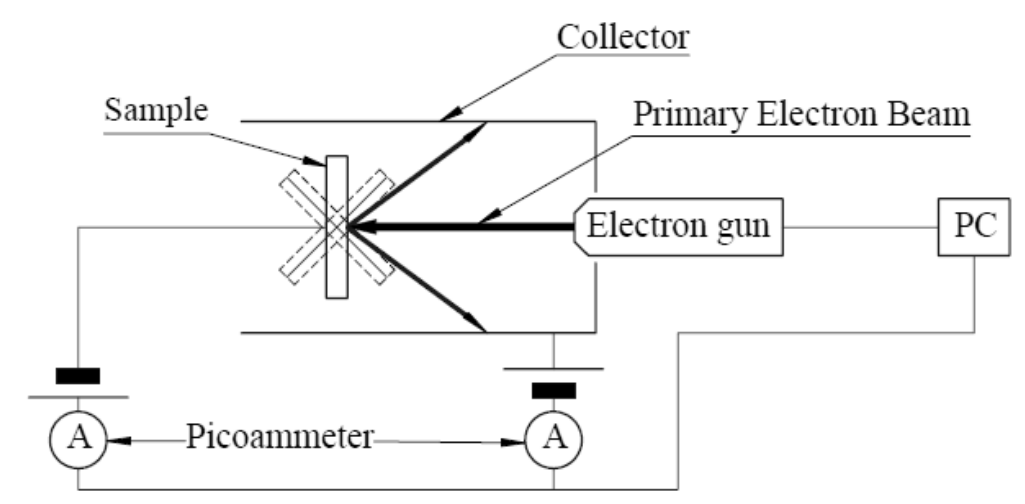

Fig. 4.14 Schematic of the experimental setup. The collector and the sample currents were measured simultaneously and the data were stored in $P C$.

To determine the $S E Y$ with the collector current method a dual channel picoammeter was used to simultaneously measure the current magnitude of the collector and sample surfaces. The connection of picoammeter with the sample and the collector was done with low noise coaxial cables. The noise level in the measurement circuits recorded on the picoammeter was in the range of $\pm 1 \mathrm{pA}$. In addition, the voltage sources were added to the electric circuit to allow biasing of the collector and the sample in order to prevent the false current readings. Current 
magnitudes were recorder in the memory buffer of the picoammeter during the measurement, after which they were transferred to $P C$ for analysis.

\subsection{Calibration measurements}

A series of calibration measurements was performed in order to obtain the values of input parameters required to maintain the magnitude and the size of the primary electron beam. Specifically, the recommended magnitude of the primary electron beam current was as low as 2 nA or lower, to avoid the processing the sample surface with electron beam during the measurements [26]. Equally important was keeping the electron beam current constant across the energy range of the primary electrons. In addition, one of the goals was to measure different characteristic surfaces on the samples, therefore the size of the beam cross-section needed to be determined and controlled. Finally, it was observed that bias voltages of sample and collector significantly affect $S E Y$ magnitude when compared to the $S E Y$ results obtained without bias voltages. To that end, the bias potentials of the collector and sample needed to be determined to control the emitted and reflected electrons and provide the accurate current measurements with minimum influence on the experiment.

\subsubsection{Primary electron beam parameters}

Uniformity of electron beam current across the primary electron energy range was necessary to maintain the comparability of the $S E Y$ results. The electron beam current magnitude was controlled by adjusting the voltage of the filament in the electron gun. Fine tuning of the beam current magnitude was controlled by the Grid and the $1^{\text {st }}$ Anode voltages of the electron gun. A gradual decrease in the electron beam current was observed for the first three hours of electron gun operation after which the current stabilized. After the beam current was leveled-off, the $S E Y$ was measured for five different filament currents with and without the applied bias 
voltages on the collector and the sample. In addition, the magnitude of the primary electron beam current was measured under the same conditions. Significant differences in $S E Y$ were observed between the measurements with and without bias voltages which was a consequence of electron movement between the surfaces of the collector and the sample. The variation of $S E Y$ at different primary electron beam currents was also observed, though it was smaller in magnitude. On the other hand, the reduction of the filament current indicated the improvement of primary electron beam current stability across the energy range of primary electrons. It was concluded that applying bias voltages and keeping the low filament current provides a more accurate measurement of $S E Y$ and a stable primary electron beam current, respectively.

The formation of free electrons, and their subsequent focusing into a beam, occurs in several steps. In an electron gun, electrons are released from the surface by applying a high current through the tantalum cathode filament and heating it up. The current in the cathode was controlled by adjusting the filament voltage in the gun power supply. Subsequently, the emitted electrons are funneled through a series of electro-optics that form and control energy, intensity, and size (cross-section) of the electron beam. Specifically, the fine tuning of the current was performed by changing the voltages of the Grid and the $1^{\text {st }}$ Anode in the electron gun. For example, increasing the potential of the Grid reduces the beam current, while the $1^{\text {st }}$ Anode increases the beam current. In addition, the size of the electron beam was controlled by adjusting the Focus voltage. Focus voltage magnitudes controlling the size of the electron beam are also a function of Grid and $1^{\text {st }}$ Anode voltages. 


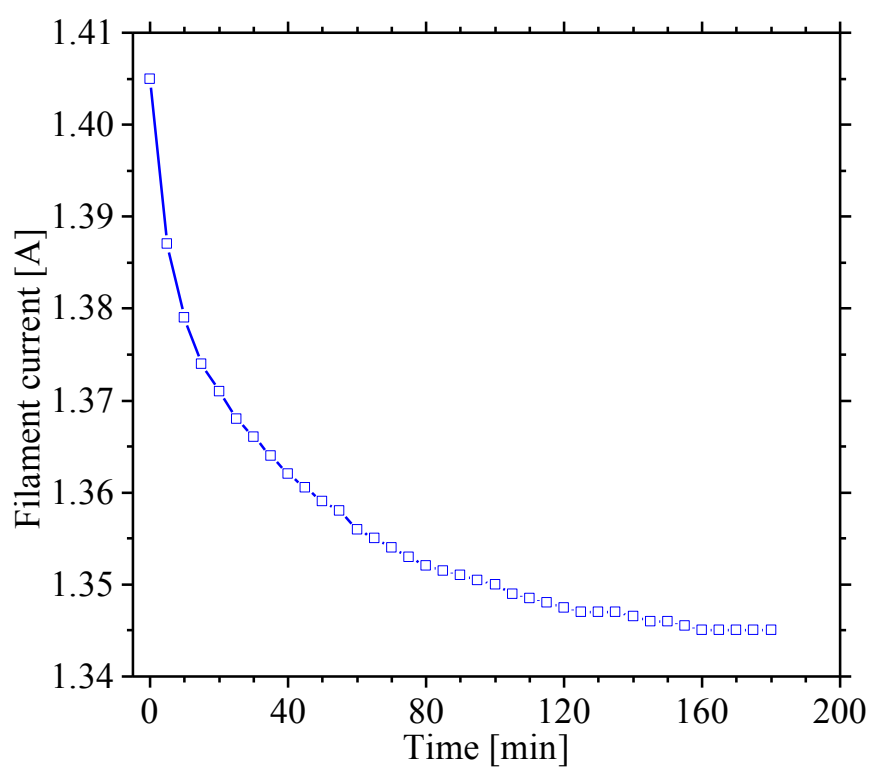

Fig. 4.15 Electron gun filament current at a constant filament voltage of 1.1 V.

Once the filament voltage was set, a decrease in the filament current was observed during the initial operation of the electron gun. Over time, the filament current gradually reduces until it becomes stable after three hours of operation (Fig. 4.15). In order to obtain stable conditions during the experiments, a uniform filament current must be achieved before beginning measurements. For that purpose, an additional warm-up sample was added to the sample stage, which was specifically used to receive the electrons from the electron gun during the filament current stabilization. The warm-up sample was positioned in front of the electron gun during the first three hours of operation. 


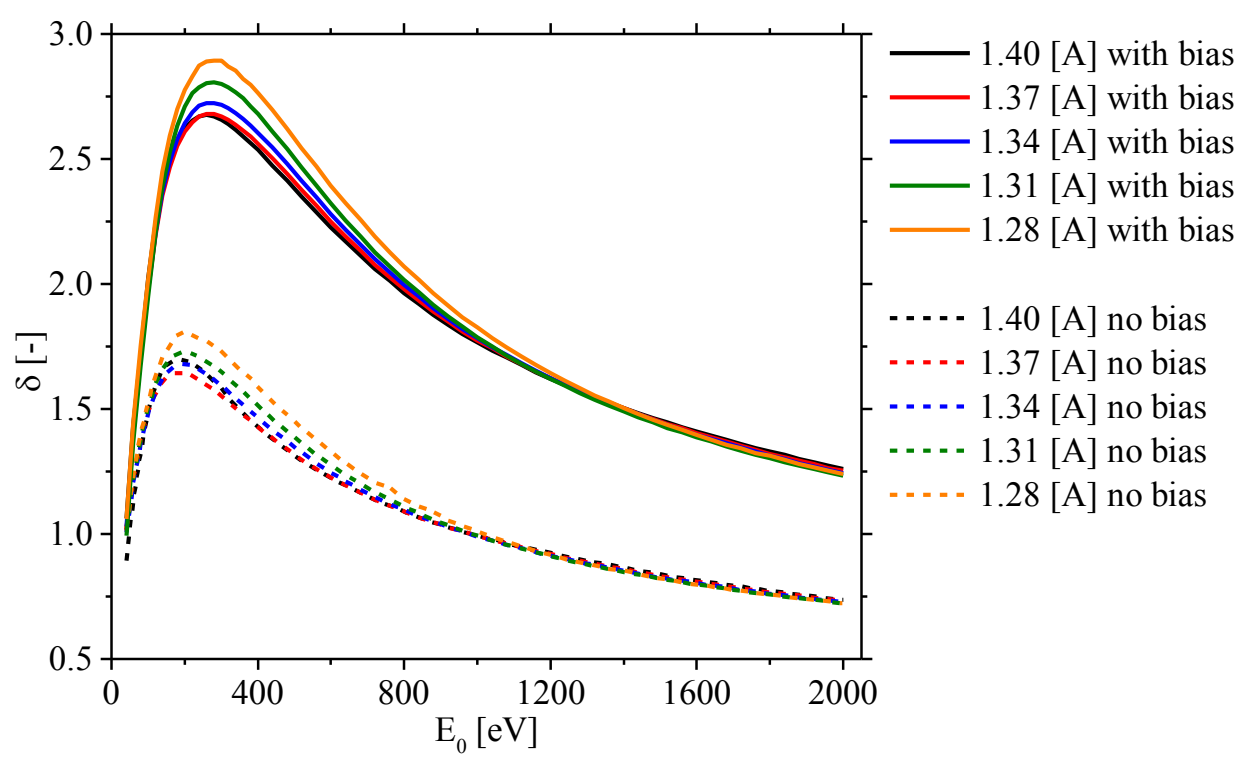

Fig. 4.16 $S E Y$ as a function of primary electron energy $\left(E_{0}\right)$ for five different electron gun filament currents, with bias $\left(U_{c}=+40 \mathrm{~V}\right.$ and $\left.U_{s}=-20 \mathrm{~V}\right)$ and without bias $\left(U_{c}=0 \mathrm{~V}\right.$ and $\left.U_{s}=0 \mathrm{~V}\right)$.

The secondary electron yield and the primary electron beam current in the energy range from 40 to $2000 \mathrm{eV}$ was determined for five different filament currents, while the Grid and $1^{\text {st }}$ Anode voltages were kept at $0 \mathrm{~V}$. Also, two sets of measurements were taken, with and without the bias voltages on the collector $\left(U_{c}=+40 \mathrm{~V}\right)$ and sample $\left(U_{s}=-20 \mathrm{~V}\right)$. The secondary electron yield and the primary electron beam current results are shown in Fig. 4.16 andFig. 4.17, respectively. Results presented in Fig. 4.16 clearly indicate that the yield values were higher when the bias voltages were applied. By setting the negative sample and the positive collector potentials, the emitted and reflected electrons from the sample surface were drawn in and absorbed by the collector, preventing formation of the tertiary electrons. The secondary electron yield results, both with and without applied bias voltages, exhibited variation as a function of the primary electron beam current. The effect of bias voltages on the various magnitudes of the electron beam current is shown in Fig. 4.17. The effect of the bias voltages on the primary 
electron beam decreased with the reduction of filament current. Moreover, the uniformity of the primary electron beam current was gradually achieved by the reduction of the filament current, as well. To summarize, lower filament parameters produce uniform primary electron beam current, which was not affected by the bias voltages. However, the measured $S E Y$ was still affected by the bias voltages of the collector and sample. In other words, the yield measured without the bias voltages on the collector and sample may be misleading since nothing was stopping the electrons from making multiple impacts until they were reabsorbed by one of the surfaces.



Fig. 4.17 Primary electron beam current $\left(i_{0}\right)$ as a function of primary electron energy $\left(E_{0}\right)$ for five different electron gun filament currents, with bias $\left(U_{c}=+40 \mathrm{~V}\right.$ and $\left.U_{s}=-20 \mathrm{~V}\right)$ and without bias $\left(U_{c}=0 \mathrm{~V}\right.$ and $\left.U_{s}=0 \mathrm{~V}\right)$.

Based on the performed measurements, it was concluded that it was necessary to employ bias potentials of the collector and the sample in order to accurately determine the SEY. Applied 
bias voltages direct the emitted electrons from the sample surface towards the collector surface where they are absorbed, preventing multiple impacts of electrons and false current readings. On the other hand, uniformity of the primary electron beam current was significantly improved by reducing the filament current levels. Subsequent calibration measurements were performed taking into the account results obtained here.

\subsection{2 $S E Y$ as a function of the collector and sample bias voltage}

A series of experiments was performed to determine the minimum values of bias potentials, as not to perturb the system more than necessary, in order to obtain the accurate $S E Y$ measurements. After a thorough literature review it was found that were no reported results of systematic measurement on the effect of the bias voltage magnitudes on the $S E Y$. To avoid unnecessary perturbation of the measurement system, it was necessary to determine the minimum values of sample and collector voltages that would produce steady $S E Y$ magnitudes. Bias voltages were varied from 0 to $50 \mathrm{~V}$ in $10 \mathrm{~V}$ increments of positive collector and negative sample bias. The secondary electron yield was determined at 100, 200, 300, and $400 \mathrm{eV}$ of primary electron beam energy for all combinations of the collector and sample potentials. The obtained results indicated that $S E Y$ magnitudes stabilized above $10 \mathrm{~V}$ of the positive collector voltage and the negative sample voltage. The best repeatability of results was observed for $+30 \mathrm{~V}$ of collector voltage and $-10 \mathrm{~V}$ of sample voltage, which were adopted as $S E Y$ measurement parameters.

In order to determine the minimum potentials that would produce stable $S E Y$ results, a series of measurements was performed at four different primary electron beam energies. At each primary electron beam energy, the collector was biased in the range from 0 to $+50 \mathrm{~V}$ in $10 \mathrm{~V}$ increments. For each collector potential value the sample bias was set in the range from 0 to -50 
$\mathrm{V}$, also in $10 \mathrm{~V}$ increments. The surface plots of the $S E Y$ at four different energies of the primary electron beam are given in Fig. 4.18. It can be observed that the yield values almost completely stabilized above the collector voltage of $+10 \mathrm{~V}$ and below the sample voltage of $-10 \mathrm{~V}$, except at $E_{0}=100 \mathrm{eV}$. At $100 \mathrm{eV}$ of primary electron energy, the increase in the negative voltage on the sample past $-10 \mathrm{~V}$ leads to gradual decrease in the measured $S E Y$ for a steady collector voltage. The most uniform results of $S E Y$ were observed for $+20 \mathrm{~V}$ and $+30 \mathrm{~V}$ of the collector bias, which were selected as possible collector voltages for further testing.
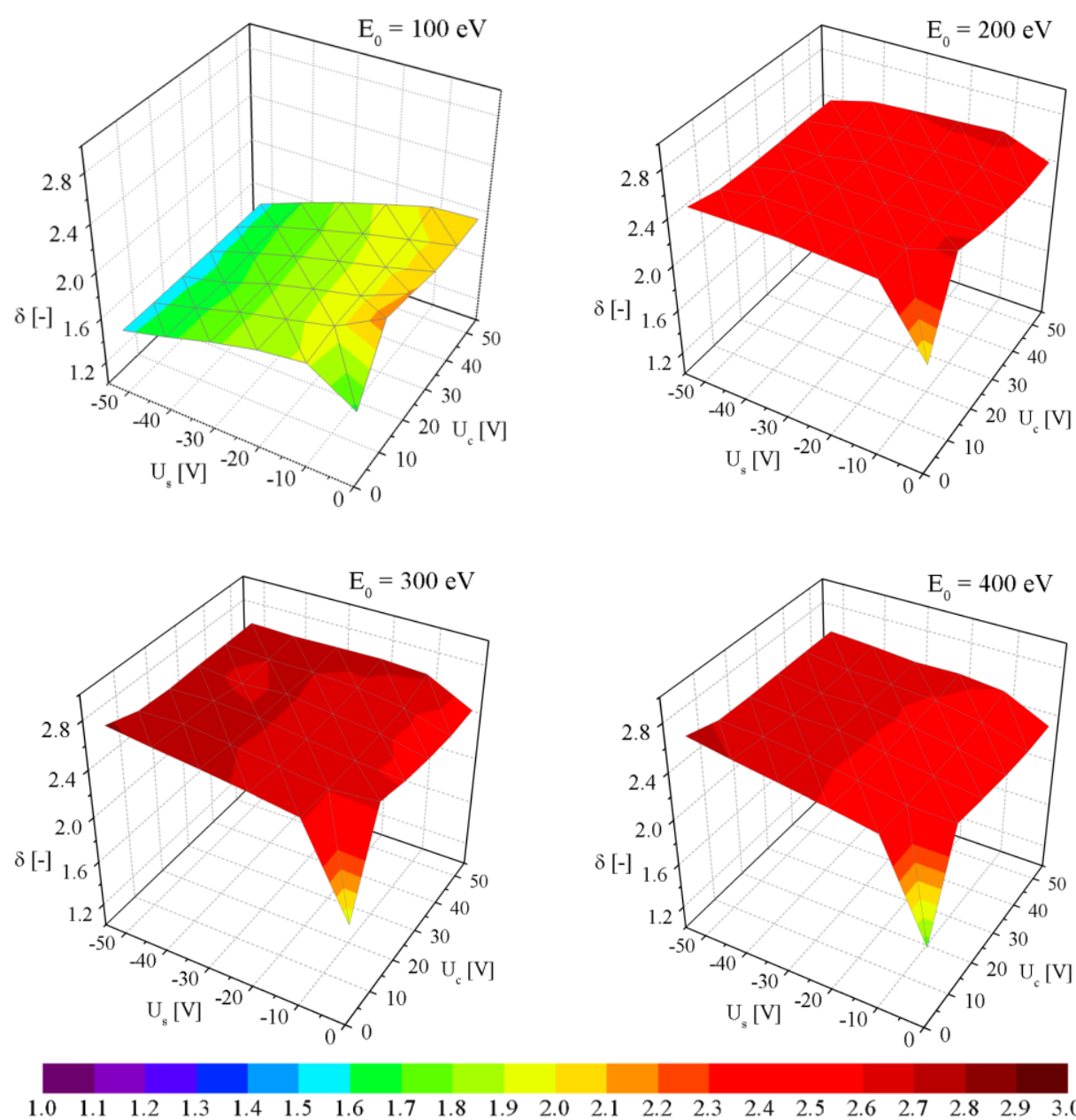

Fig. 4.18 $S E Y$ as a function of the collector $\left(U_{c}\right)$ and the sample $\left(U_{s}\right)$ voltages for four primary electron beam energies $\left(E_{0}\right)$. 

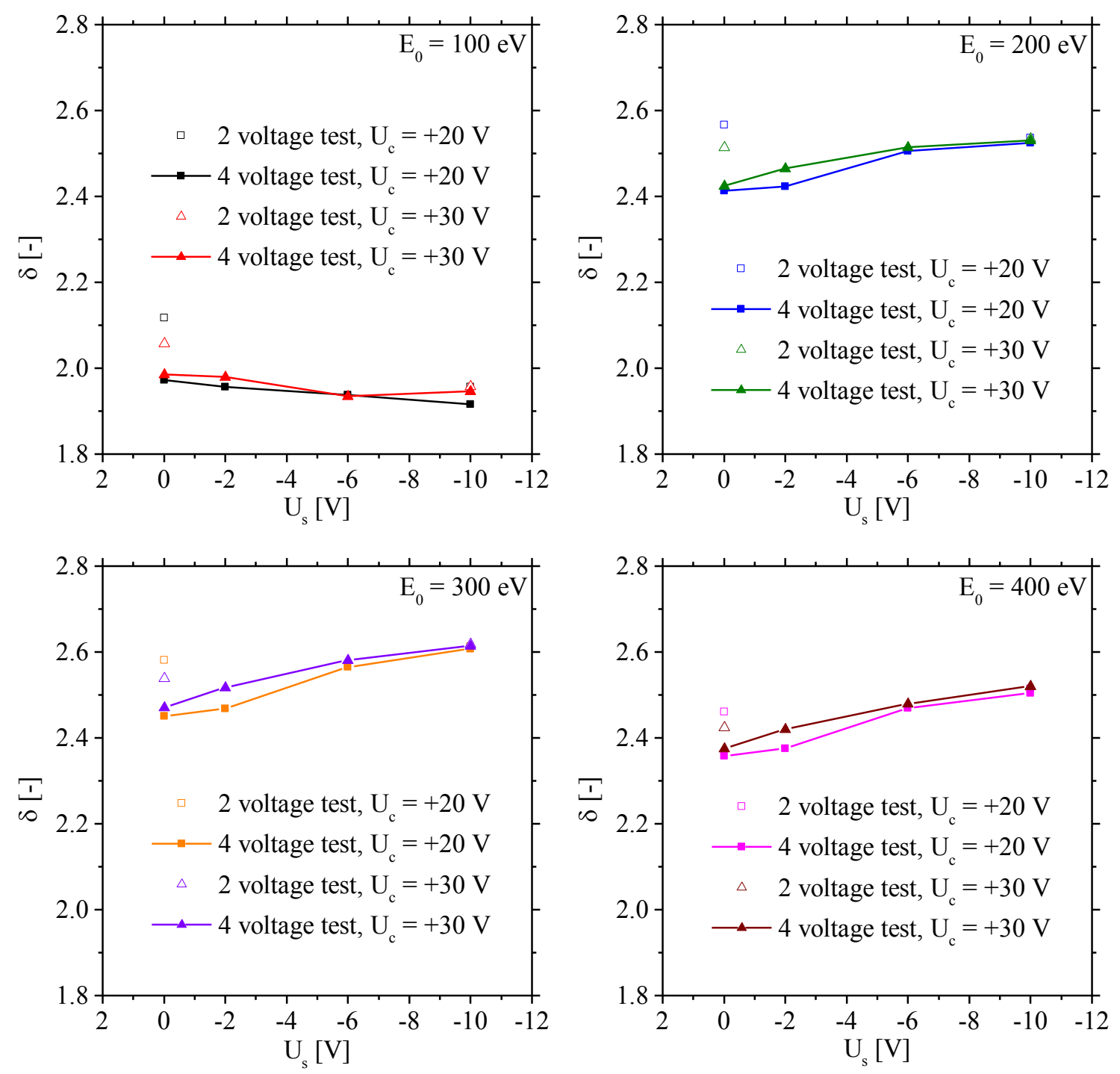

Fig. 4.19 $S E Y$ as a function of the sample bias voltage $\left(U_{s}\right)$ at two collector potentials $\left(U_{c}\right)$ for four primary electron beam energies $\left(E_{0}\right)$.

Based on the results, two voltage levels were selected as the possible bias of the collector $(+20 \mathrm{~V}$ and $+30 \mathrm{~V})$. Even though it was established that the yield values were stable at the bias voltages of the sample below $-10 \mathrm{~V}$, it is beneficial to have the voltage of the sample as close to zero as possible to reduce electron beam deflection when determining the influence of the primary electron beam incident angle on the $S E Y$. For that purpose, additional tests were 
performed in the range between the $0 \mathrm{~V}$ and $-10 \mathrm{~V}$ of the sample voltage. Specifically, yield values were determined at the same four primary electron beam energy levels as before, at $0,-2$, -6 , and $-10 \mathrm{~V}$ sample bias, and two collector voltages at $+20 \mathrm{~V}$ and $+30 \mathrm{~V}$. The $S E Y$ measured at these voltages was compared with the previous yield results at $0 \mathrm{~V}$ and $-10 \mathrm{~V}$ on the sample (Fig. 4.19). Additional test points confirmed that the effect of the sample bias could be described as linear in the range up to $-10 \mathrm{~V}$. The measured yields converged at approximately the same value at $-10 \mathrm{~V}$ of the sample bias and both $+20 \mathrm{~V}$ and $+30 \mathrm{~V}$ of the collector bias. In order to make the final decision on the collector bias voltage, two $S E Y$ measurements were made in the primary electron energy range from 40 to $400 \mathrm{eV}$ at both collector voltages (Fig. 4.20). The collector voltage was selected to be $+30 \mathrm{~V}$, since the SEY measurements indicated better repeatability at that collector bias value.

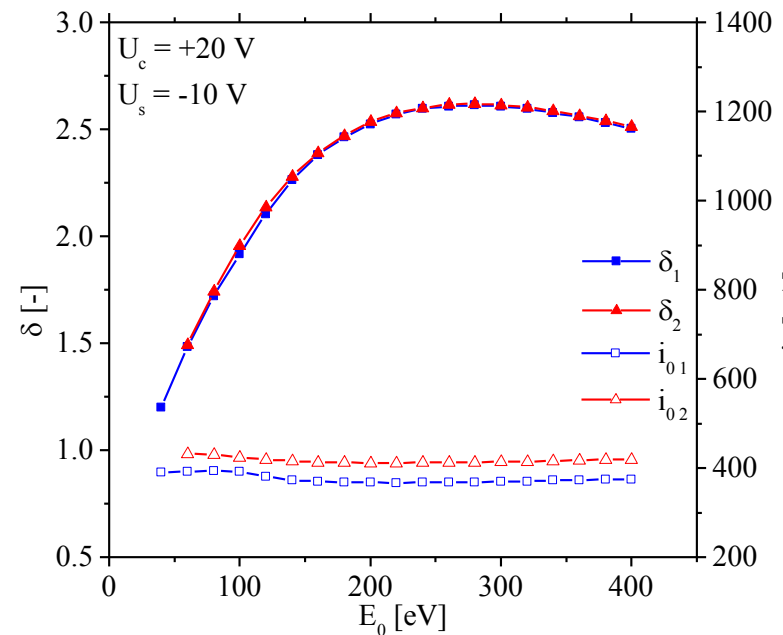

(a)

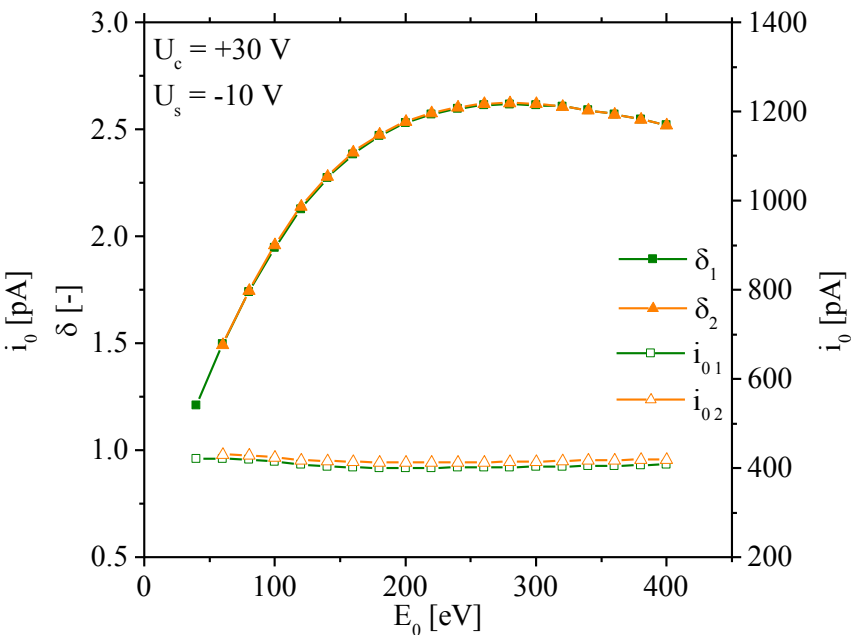

(b)

Fig. 4.20 SEY and primary electron beam current $\left(i_{p}\right)$ as a function of $E_{0}$, for (a) $U_{c}=+20 \mathrm{~V}$ and $U_{s}=-10 \mathrm{~V}$, and (b) $U_{c}=+30 \mathrm{~V}$ and $U_{s}=-10 \mathrm{~V}$. 
A systematic approach was adopted to determine the influence of bias voltages of the sample and the collector on SEY magnitude. A secondary electron yield was determined at four different energy levels and voltage ranges from 0 to $+50 \mathrm{~V}$ on the collector and 0 to $-50 \mathrm{~V}$ with $10 \mathrm{~V}$ increments. The obtained results pointed out that the $S E Y$ stabilizes above $+10 \mathrm{~V}$ of collector bias and below $-10 \mathrm{~V}$ of sample bias. In addition, the steadiest results were observed for $+20 \mathrm{~V}$ and $+30 \mathrm{~V}$ of collector bias and for $-10 \mathrm{~V}$ of sample bias. Two additional SEY measurements performed in the primary electron energy range of 40 to $400 \mathrm{eV}$ indicated better repeatability of the results for $+30 \mathrm{~V}$ of the collector bias. To summarize, the optimal bias voltages obtained by the tests were chosen at $+30 \mathrm{~V}$ and $-10 \mathrm{~V}$ of the collector and the sample, respectively, and adopted as the $S E Y$ measurement parameters.

\subsubsection{Primary electron beam size control}

To determine the $S E Y$ of characteristic surfaces of the fabricated samples, the primary electron beam size needed to be determined and controlled. Using a phosphor screen that illuminates in the area where the electrons are impacting is a common method to ascertain the size of the electron beam. An attempt was made to measure the size of the electrons beam by using a phosphor screen (Kimball Physics PHOS-UP22SS-B5x5-R500), but the low current of the beam was not able to excite the phosphor surface. However, once the current magnitude was increased, a blue dot on the screen was observed. Since this method was not effective in measuring the beam size at low current, the method described in [26] was adopted and adapted to fit the experimental setup. The schematic of developed primary beam collector $(P B C)$ is shown in Fig. 4.21. The size of the electron beam was controlled by adjusting the Focus voltage of the electron gun to funnel as many electrons as possible through the aperture on the front plate of the $P B C$. When the measured current on the front plate was zero, the electron beam diameter was 
smaller than the aperture diameter. The magnitudes of the Focus voltage were determined for the primary electron energies in the range from 60 to $2000 \mathrm{eV}$.

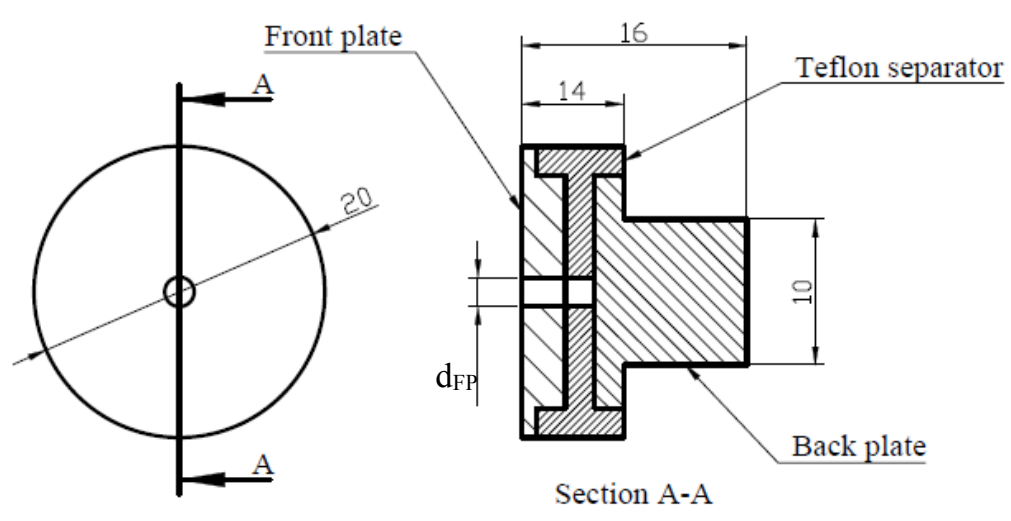

Fig. 4.21 Design sketch of the Primary Beam Collector $(P B C)$.

The primary beam collector $(P B C)$ was designed, fabricated, and tested to measure the size of the electron beam cross-section. The design of the $P B C$ included the back and the front plate made of stainless steel, and a Teflon separator was used as an electrical insulation between the two plates. Two front plates were fabricated, with a different diameter circular opening at its center ( 2 and $3 \mathrm{~mm}$ ), to determine the quantity of electrons passing through the aperture for the same electron gun parameters. In order to measure the beam size, the $P B C$ was attached to the sample stage and centered in front of the electron gun. Specifically, as the primary electron beam leaves the mouth of the electron gun, it passes through the aperture on the collector and hits the center of the $P B C$. All components along the path of the primary electron beam were biased to successively larger positive potentials. For instance, the collector was placed at $+25 \mathrm{~V}$, the front plate of $P B C$ at $+30 \mathrm{~V}$ and the back plate of $P B C$ at $+50 \mathrm{~V}$. The purpose of these potentials was to prevent emission of secondary electrons from interfering with the beam size measurement. 
The picoammmeter was used to measure the current on the front and the back plate of the $P B C$. When the front plate current was zero, the electron beam cross-section was smaller than the aperture of the front plate. The size of the electron beam was adjusted to fit the size of the front plate aperture. The fraction of electrons reaching the back plate through the aperture on the front plate was calculated as a ratio of the back plate, and the sum of the back and the front plate currents. This quantity presented the part of the beam hitting the back plate surface of the $P B C$. The equation used to determine the ratio of currents was set as

$$
A=\frac{i_{B P}}{i_{B P}+i_{F P}},
$$

where $i_{B P}$ was the back plate current and $i_{F P}$ was the front plate current. The ratio was measured using both front plates with different diameters. After several iterations, Filament, Grid and $1^{\text {st }}$ Anode voltages of the electron gun were selected that formed the electron beam size needed to measure the $S E Y$ of the characteristic surfaces of the samples. It is important to note that these parameters were chosen as a trade-off between the beam size and the primary electron beam current magnitude across the energy range. The electron beam parameters used for the experiments are shown in Table 4.3. The Focus voltage magnitudes and the corresponding electron beam size as a function of the primary electron energy are given in Fig. 4.22.

Table 4.3 The electron beam parameters used for the $S E Y$ measurements.

\begin{tabular}{cc}
\hline $\mathrm{e}^{-}$gun parameters & Magnitude \\
\hline Source Voltage [V] & 1.1 \\
Source Current [A] & 1.345 \\
Grid [V] & 16 \\
$1^{\text {st }}$ Anode [V] & 93 \\
Distance to the sample [mm] & 27 \\
\hline
\end{tabular}




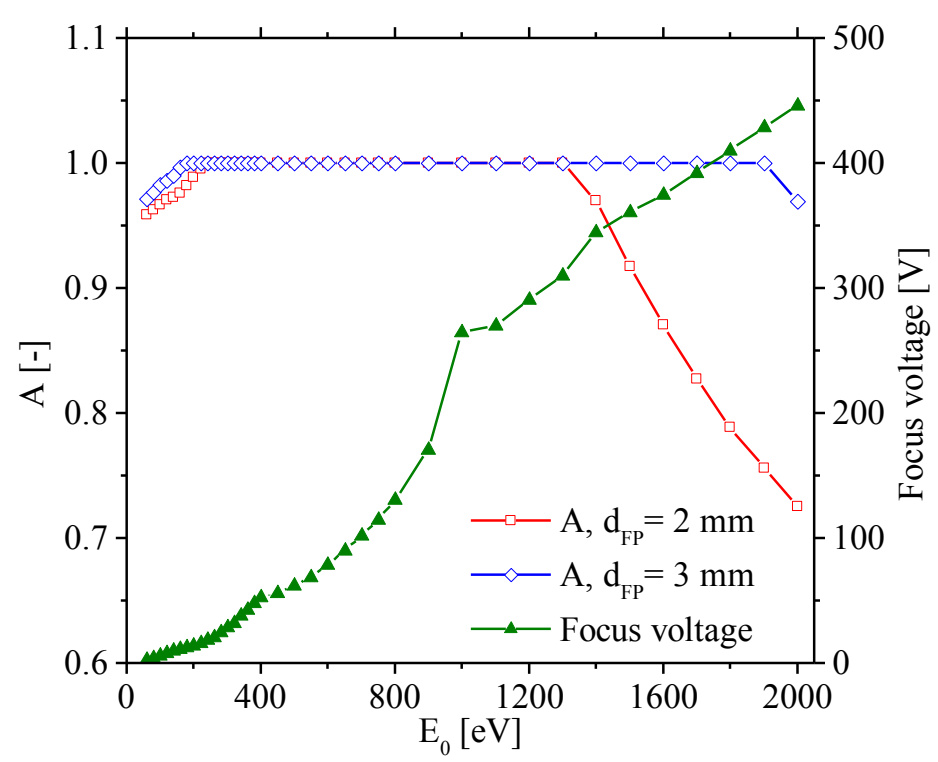

Fig. 4.22 The ratio of electron currents $(A)$ for two diameters of the front plate hole $\left(d_{F P}\right)$ and Focus voltage, as a function of the primary electron energy $\left(E_{0}\right)$.

The primary electron energy range of 60 to $2000 \mathrm{eV}$ was divided into three sections with different incremental increases of the energy. The first section of the primary electrons energy from 60 to $400 \mathrm{eV}$ was incrementally increased by $20 \mathrm{eV}$. In the second section, 400 to $800 \mathrm{eV}$ of the primary electron energy increased in $50 \mathrm{eV}$ increments. Last, the third section of the primary electron energy from 800 to $2000 \mathrm{eV}$ was scanned with $100 \mathrm{eV}$ increments. With the electron beam directed towards the surface center of the $P B C$, the current was measured on the front and back plate with the picoammeter. At each primary electron beam energy, the Focus voltage magnitude (Fig. 4.22) was adjusted to the value that provided the minimum current on the front plate. When the front plate current was equal to zero, all electrons of the primary electron beam were going through the aperture of the front plate and impacting the surface of the back plate. In Fig. 4.22, for the Focus voltage function, ratio $A$ was determined for two diameters of the front plate aperture. For an aperture diameter of $2 \mathrm{~mm}$, at primary electron energy of 60 
$\mathrm{eV}$ the value of ratio $A$ was 0.95 indicating that $95 \%$ of the beam electrons are within the aperture diameter. The ratio $A$ value increases almost linearly to value 1 with the increase of the primary electron energy to $220 \mathrm{eV}$ where $100 \%$ of electrons are going through a $2 \mathrm{~mm}$ aperture. In the primary electron beam energy range from 1300 to $2000 \mathrm{eV}$, ratio $A$ value starts decreasing from 1 to 0.73 . The measurement was repeated for the front plate aperture diameter of $3 \mathrm{~mm}$. The ratio $A$ value starts at 0.97 at $60 \mathrm{eV}$, rises to 1 at $160 \mathrm{eV}$, remains constant up to primary electron energy of $1900 \mathrm{eV}$, and reduces to 0.97 at $2000 \mathrm{eV}$. The Focus voltage as a function of the primary electron beam energy was adopted for $S E Y$ measurements.

The cross-section of the electron beam obtained by applied the Focus voltage was less than $3 \mathrm{~mm}$ across most of the energy range which allowed the measurement of the characteristic surfaces in the weld area of fabricated samples. The known size of the electron beam also permitted the measurement of multiple spots on the sample without overlapping the irradiated surfaces of the measured spots.

\subsection{Measurement procedure and data analysis}

A measurement procedure was established to maintain the comparability of the results during the $S E Y$ measurements. All $S E Y$ measurements were performed using the same electron beam parameters, sample and collector voltages, the distance of the measuring spot from the mouth of the electron gun, at all incident angles and energy range of the primary electron beam. To mitigate the effect of the current noise levels in the measurement circuits, the primary electron beam current was kept between the 300 and 500 pA. By keeping the current in this range, the influence of the current noise levels on the $S E Y$ measurements was less than $1 \%$ for all primary electron energy values. In addition, the $S E Y$ magnitude was measured three times over the period of three seconds for a single primary electron energy. Each $S E Y$ magnitude showed a 
high level of mutual agreement. A single $S E Y$ curve was determined as an average value of these three magnitudes. The average $S E Y$ curve of the characteristic surface was calculated based on the SEY measured at different spots of the characteristic surface. Since the sample surfaces represent only a section of the characteristic surfaces, the variation of results was presented by calculating the sample standard deviation. The sample standard deviation of SEY is used to calculate the deviation of the entire cavity surface based on the variation of the sample surface. Based on sample standard deviation, the range of $S E Y$ of characteristic surfaces of the entire cavity were inferred by calculating the prediction interval with probability value of 0.9 . The described data analysis was performed for all incident angles of the primary electron beam.

The measurement procedure in this study was performed as follows. First, the base vacuum of $2 \cdot 10^{-9}$ Torr was achieved, and a warm-up sample was placed in front of the electron gun during the filament current stabilization. Second, every component of the experimental setup was powered up and was left at its lowest setting for a half an hour to stabilize the operation of electronic parts. Third, the bias voltages on the collector and the sample were gradually adjusted to $+30 \mathrm{~V}$ and $-10 \mathrm{~V}$, respectively. Fourth, the parameters of the electron gun were steadily increased together to their respective values (Table 4.3), and the primary electron beam energy was set to $20 \mathrm{eV}$ for the duration of filament warm-up. Once the filament current stabilized, the electron beam current was blocked remotely by using a beam cutoff command in LabView software. While the beam was blocked, the samples were positioned using the sample manipulator to the predefined measuring spots. Afterwards, the primary electron beam was released and the measurement was initiated. All the $S E Y$ curves were taken in 60 to $2000 \mathrm{eV}$ energy range of the primary electrons. The primary electron energy range was divided into three sections with a different incremental increase in energy. Specifically, in the section from 60 to 
$400 \mathrm{eV}$, the incremental increase of the primary electron energy was $20 \mathrm{eV}$, the following energy section from 400 to $800 \mathrm{eV}$ was increased by $50 \mathrm{eV}$ increments, and the last energy section from 800 to $2000 \mathrm{eV}$ had energy increased by $100 \mathrm{eV}$. After the scan of the entire energy range was recorded, a sample under examination was replaced by another one. The measured sample and collector currents were recorded in the memory buffer of the picoammeter. At the end of the measurement, the data were transferred to the $P C$ before starting the following measurement.

In order to provide a better understanding of the $S E Y$ dependence on primary electron energy beam, angle of incidence, and the influence of plasma processing, measurements were repeated at multiple points across the sample surface. No more than three test spots were measured consecutively on a single sample in order to avoid warming up the surface which may affect the $S E Y$ magnitude. After the measurement was completed on the test spot, the primary electron beam was cut off until the next position was set. Tests were performed on samples at normal and $\pm 15^{\circ}$, followed by $+30^{\circ},+45^{\circ}$, and $+60^{\circ}$, and finishing the set with the samples at $30^{\circ},-45^{\circ}$, and $-60^{\circ}$ of the primary electron beam incident angle. Once all the measurements were completed, a warm up sample was placed in front of the electron gun and parameters were gradually reduced to zero before powering down the electron gun.

Three SEY curves were determined based on the recorded measurements (Fig. 4.23). A single $S E Y$ curve was calculated as an average of the three recorded values. All three curves showed high mutual agreement of results, indicating that the noise levels in the measurement circuits did not influence the results of measurement. 


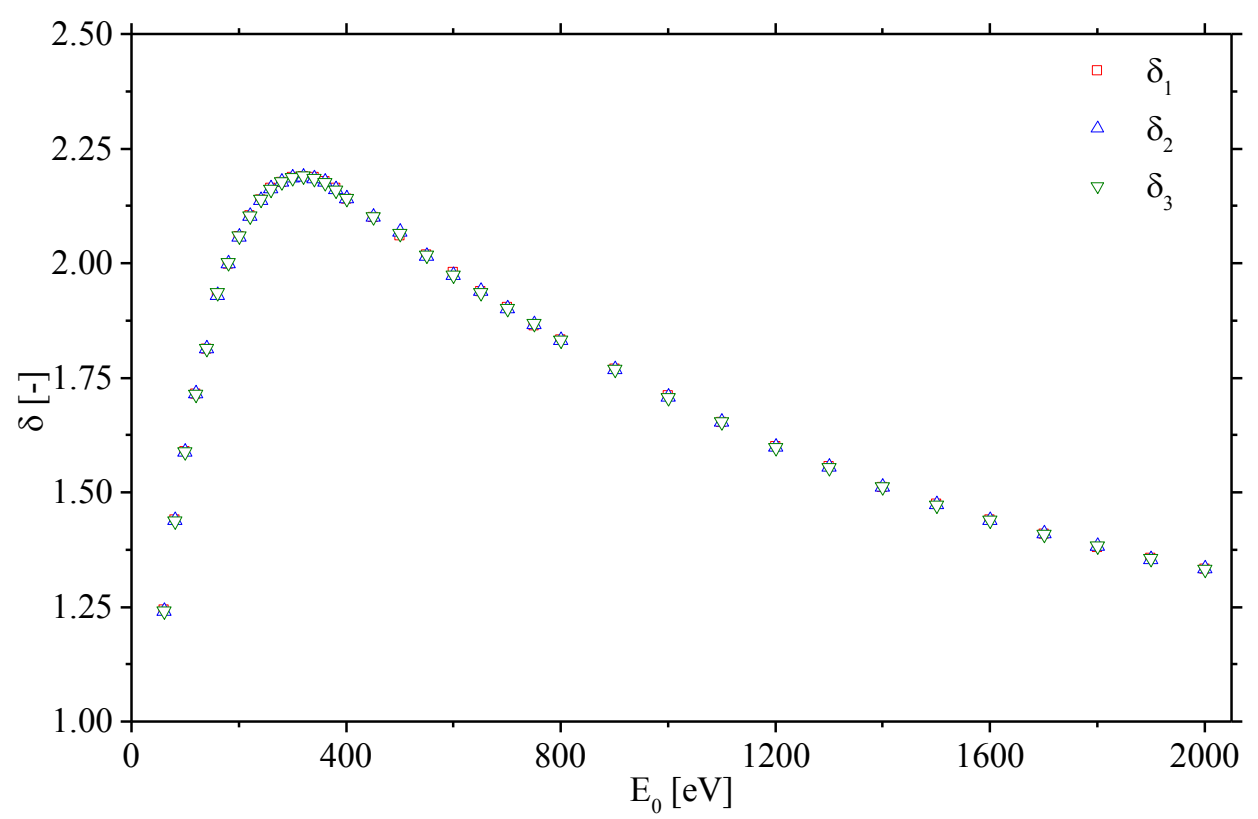

Fig. 4.23 Three SEY curves recorded during an energy scan of a single measurement spot as a function of the primary electron beam energy.

Several different metrics have been used to represent the experimental results, namely the arithmetic mean, the sample standard deviation, and the $90 \%$ prediction interval.

First, an arithmetic mean was used to present the average $S E Y$ value recorded on the characteristic surface, which was calculated as

$$
\bar{\delta}=\frac{1}{n} \cdot \sum_{i=1}^{n} \delta_{i}
$$

where: $\bar{\delta}$ is the average value of the yield at a primary electron energy, $n$ is the total number of measurements performed on a characteristic surface, and $\delta_{i}$ is the yield measured at a primary electron energy during a single test.

Second, a sample standard deviation was calculated. Namely, the samples are fabricated to represent the entire surface of the cavity, however, the $S E Y$ was determined only on a minor area of the sample. In order to represent the yield variation of the entire characteristic surface of the 
cavity based only on the limited number of tests performed on a sample, a corrected sample standard deviation $s$ is calculated as

$$
s=\sqrt{\frac{1}{n-1} \cdot \sum_{i=1}^{n}\left(\delta_{i}-\bar{\delta}\right)^{2}}
$$

Last, the prediction interval is an estimate of an unknown $S E Y$ value $\left(\delta_{\mathrm{n}+1}\right)$ based on already measured data with a certain probability. In this work, prediction intervals indicate the range of yield values that can be measured on the characteristic surfaces outside of the sample area with the 0.9 probability. This interval was calculated as

$$
\bar{\delta}-t_{0.05, n-1} \cdot s \cdot \sqrt{1+\frac{1}{n}} \leq \delta_{n+1} \leq \bar{\delta}+t_{0.05, n-1} \cdot s \cdot \sqrt{1+\frac{1}{n}}
$$

where $t_{0.05, \mathrm{n}-1}$ is the two tail probability of a normal distribution [55].

The effect of the incident angle of primary electrons on $S E Y$ was described in [27,31], but neither of the equations consider the shift of the primary energy at which the maximum yield occurs. An angular term in both reported equations was a function of a single parameter introduced as a material characteristic. Since both equations produce similar results, only the equation from [27] was considered in the analysis of the experimental results that describe the influence of primary electron beam incident angle $(\theta)$ on the $S E Y$. The material characteristic parameter can be determined by minimizing the sum of squares of residual between the average yield at each incident angle and the predicted yield value. The equation from [27] for the $S E Y$ as a function of incident angle of primary electrons is

$$
\hat{\delta}\left(\theta, E_{0}\right)=\bar{\delta}\left(0, E_{0}\right) \cdot(\cos \theta)^{-\eta}
$$


The value of the fitted parameter was compared for each characteristic surface, before and after plasma. The results were used to evaluate the effect of plasma processing on the angular distribution and variation of the $S E Y$ at primary electron beam energy of $320 \mathrm{eV}$.

The described measurement procedure was adopted to maintain the comparability of the $S E Y$ results obtained from the developed experimental setup. The parameters of the electron gun, sample position, and voltage potentials of the collector and sample were kept steady for the duration of the $S E Y$ measurements. The primary electron beam energy was incrementally increased from 60 to $2000 \mathrm{eV}$ and the collector and the sample currents were recorded. Based on the measured currents, $S E Y$ was determined on each of the measuring spots. The average $S E Y$ of the characteristic surfaces were determined based on the measurements obtained from the separate measurement spots. The variation of the $S E Y$ magnitudes was accounted by calculating the sample standard deviation. Furthermore, the expected $S E Y$ magnitudes across the characteristic surfaces of the cavity were inferred by calculating the prediction interval with a probability level of 0.9 .

\subsection{Plasma processing}

The effect of plasma processing on the $S E Y$ of niobium samples was determined by exposing the samples to plasma formed in commercial plasma processing system (Plasma Etch PE-25). Two different processing gases were employed in order to test the effect they had on the $S E Y$ of the sample surface. Samples were exposed to nitrogen and argon/oxygen plasma for one hour at 50 mTorr pressure. After processing, the samples were left in a vacuum for one hour to cool-down. During the transfer to the $S E Y$ measurement chamber samples were exposed to air at atmospheric pressure for one hour before the vacuum chamber was closed. After reaching the 
base vacuum pressure, SEY measurements were repeated and the results were compared to determine the effect of the plasma processing.

Plasma processing is the method used for removing the contamination and modifying the surface exposed to the ionized gas at low pressures. Plasma employed in surface processing is usually created at a low vacuum (mTorr range) using several power sources, namely direct current $(D C)$, radio-frequency $(r f)$, or microwave $(M W)$. Under the influence of the applied electric field, free electrons are accelerated past the threshold for ionization. As a consequence, these electrons impact neutral atoms or molecules and create electron-ion pairs, and with the presence of neutral radicals, the processing of the surface was achieved by either a chemical reaction or a mechanical interaction of plasma with the surface. For the cleaning effect, ionized atoms or molecules of the processing gas interact with the non-metallic impurities and the interaction products are removed from the surface and the system. The applied processing gas determines the removal method and the type of pollutants affected. For example, the use of inert gases will remove the surface impurities by physically dislodging them from the surface due to energy transfer. On the other hand, active gases, like oxygen, hydrogen or chlorine, will chemically react with the contaminants or the substrate and form volatile molecules that can be removed from the processing chamber.

A commercial plasma processing system was used to process the samples. The apparatus was relatively simple to operate and allowed the use of different processing gases. Plasma generation was controlled by a continuously variable $r f$ power supply with an automatic matching network and a manual gas flow valve. The parameters used to process the samples remained the same for all processed sample sets. Specifically, the power supply was set to its maximum value of $100 \mathrm{~W}$ at $50 \mathrm{kHz}$, while the pressure in the processing chamber during 
plasma processing was 50 mTorr. The gases used for processing were $\mathrm{N}_{2}$ and $\mathrm{Ar} / \mathrm{O}_{2}$ mixture. One set of three samples was exposed to $\mathrm{N}_{2}$, while three sets of nine samples were processed in $\mathrm{Ar} / \mathrm{O}_{2}\left(10 \% \mathrm{O}_{2}\right.$ content $)$ plasma.

The plasma processing procedure was performed in several steps, and was repeated for each set of samples and gases used. Once the samples were placed inside the processing chamber, venting was initiated and continued until the pressure reaches 20 mTorr. At that time, the processing gas was introduced, raising the pressure to 50 mTorr, which remains constant during the processing of the samples. Samples were processed in plasma for one hour. Following the plasma shut down, samples were left in the processing gas at the pressure of $50 \mathrm{mTorr}$ to cool down for an additional hour. Subsequently, the air was released into the processing chamber and the pressure increased to the atmospheric level. Exposure to the air at atmospheric pressure was one hour for both $\mathrm{N}_{2}$ and $\mathrm{Ar} / \mathrm{O}_{2}$ processed samples. The samples were then placed on the specimen stage and the vacuum chamber was closed. 


\section{CHAPTER 5}

\section{RESULTS}

The developed experimental setup was used to measure the effect of plasma processing on the $S E Y$ of characteristic surfaces found in the $S R F$ niobium cavity. The results presented in this section are organized as follows.

First, the effect of the nitrogen $\left(\mathrm{N}_{2}\right)$ plasma processing on the $S E Y$ at a normal incident angle of primary electrons was tested on the samples representing the base metal and weld zone.

Second, the effect of the argon/oxygen $\left(\mathrm{Ar} / \mathrm{O}_{2}\right)$ plasma on $S E Y$ was measured at nine incident angles of primary electrons. The $S E Y$ was measured on samples representing the base metal and weld area surfaces found on the niobium $S R F$ accelerating cavity.

A majority of the measured $S E Y$ magnitudes from characteristic surfaces converged to approximately the same average value after plasma processing. Also, an increase in the $S E Y$ was observed on most of the examined surfaces. In order to identify potential cause of the $S E Y$ increase, three surface characterization methods were used to determine the state of the surface before and after plasma processing. These are, specifically, surface roughness, surface microstructure, and chemical composition of the surface. The measurement methods included the atomic force microscope $(A F M)$, scanning electron microscope (SEM), and energy-dispersive $x$ ray spectroscopy $(E D S)$.

Third, several measurements using the retarding potential of the collector were taken at three energies of the primary electrons, in an attempt to determine the energy distribution curve $(E D C)$ of reflected electrons before and after $\mathrm{Ar} / \mathrm{O}_{2}$ plasma. 


\subsection{The $\mathrm{N}_{2}$ plasma processing results}

Three samples were used to test the effect of nitrogen plasma on the $S E Y$ of base metal and weld zone surface. Two samples of base metal were named BASE 1 and BASE 2. The surface of $B A S E 1$ sample was affected by an electron beam during the calibration of the experimental setup, while the surface of $B A S E 2$ sample was not exposed to the electron beam prior to the $S E Y$ measurements. The third sample had a weld bead across the surface and was named $W Z$ sample.

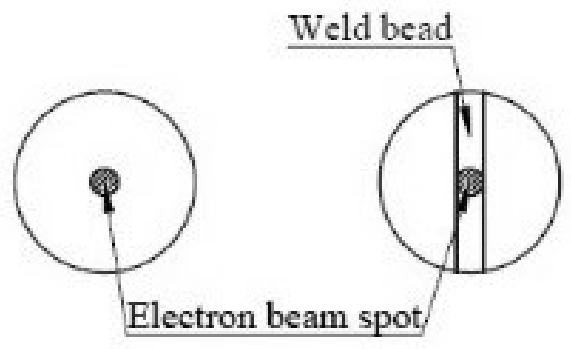

(a)

(b)

Fig. 5.1 Sketch of (a) BASE 1 and BASE 2 samples, and (b) WZ sample used for $\mathrm{N}_{2}$ plasma processing, with the location of the electron beam during $S E Y$ measurement.

Seven SEY measurements were performed on the center spot of each one of the three samples (Fig. 5.1), in order to determine the change in the SEY magnitude after repeated exposure to the electron beam. Tests were performed on samples before and after plasma treatment.

To avoid increasing the temperature of the sample surface, which could affect the $S E Y$ magnitude, no consecutive measurements were performed on the surface of a single sample. The $S E Y$ was measured in the energy range $E_{0}=60-2000 \mathrm{eV}$. In order to accurately present the changes in the $S E Y$ curve, the energy range of primary electrons was divided into the three 
regions with different incremental increase in primary electron energy. The first region of the energy range $E_{0}=60-400 \mathrm{eV}$ has a $20 \mathrm{eV}$ incremental increase. The $S E Y$ magnitudes vary significantly in this energy range and the small increment increase in energy is required to accurately determine changes in the $S E Y$. The second region of the energy range $E_{0}=400-1500$ $\mathrm{eV}$ has a $50 \mathrm{eV}$ incremental increase. Finally, the third region of the energy range $E_{0}=1500-2000$ $\mathrm{eV}$ has a $100 \mathrm{eV}$ incremental increase.

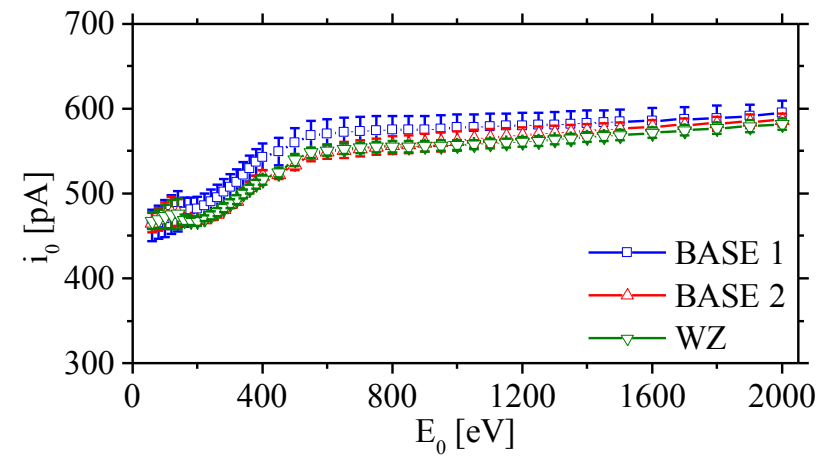

(a)

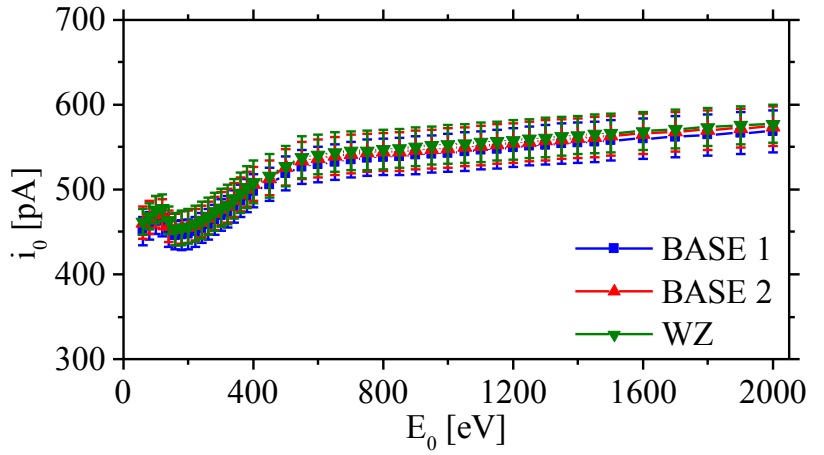

(b)

Fig. 5.2 The average primary electron beam current $\left(i_{0}\right)$ as a function of primary electron beam energy $\left(E_{0}\right)$, (a) before plasma processing [23], and (b) after plasma processing in nitrogen for $B A S E 1, B A S E 2$, and $W Z$ sample. Statistical bars are covering the standard deviation.

The average primary electron beam currents for each of the samples were compared before, and after nitrogen plasma processing (Fig. 5.2). It is apparent that two distinct regions of the curves are present. The first region of the curve, for all samples, is from 60 to $500 \mathrm{eV}$ where the beam current has a steep slope. The variation of the primary electron beam current on average was $\pm 15 \mathrm{pA}$, at each energy level, while the difference between the average current at $60 \mathrm{eV}$ and $2000 \mathrm{eV}$ did not extend above $140 \mathrm{pA}$. When the variation of the primary electron beam current 
is compared with the variations of $S E Y$ magnitudes (Fig. 5.3), it can be concluded that the variation of beam current did not significantly affect the value of $S E Y$.

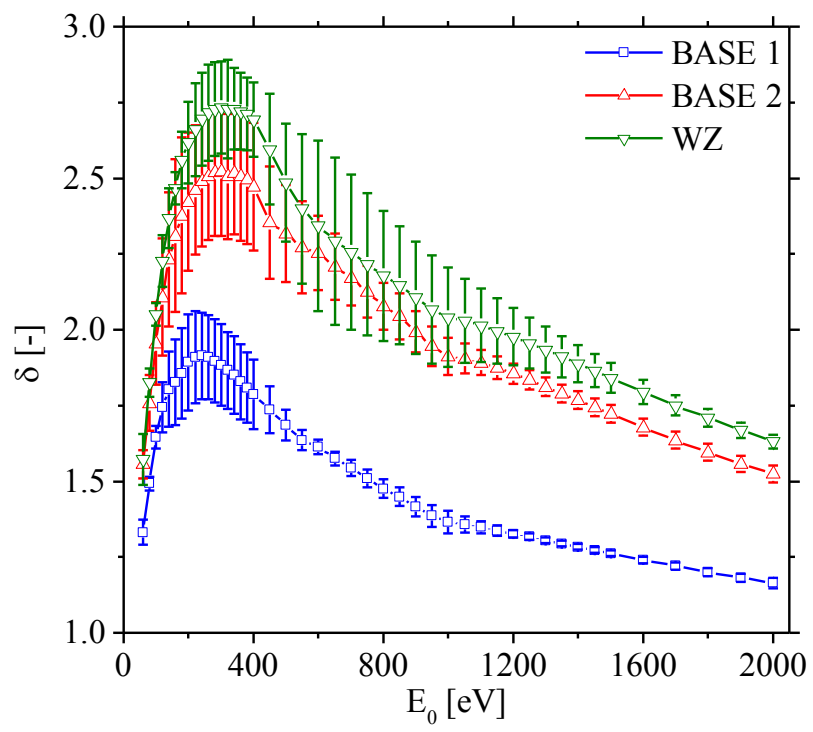

(a)

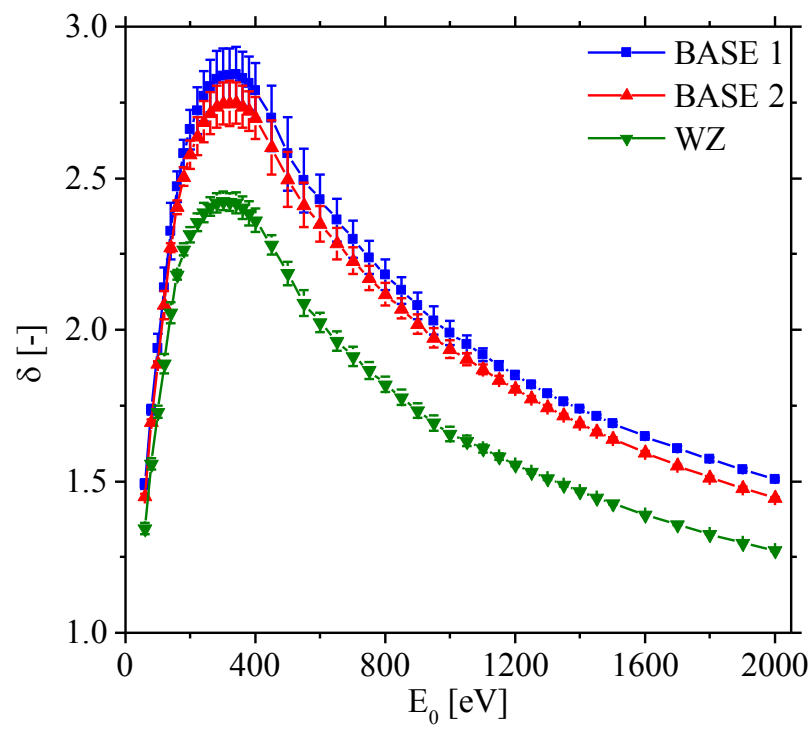

(b)

Fig. 5.3 Average $S E Y$ as a function of $E_{0}$, for $B A S E 1, B A S E 2$, and $W Z$ samples (a) before plasma processing [23], and (b) after plasma processing in nitrogen. Statistical bars are representing $90 \%$ prediction interval.

Seven measurements performed on $B A S E$ 1, BASE 2, and $W Z$ sample, before and after plasma, were used to determine the average SEY value and to calculate the corresponding statistical bars (Fig. 5.3). Before plasma processing, BASE 2 and $W Z$ samples have shown a slow but steady decrease in SEY after each measurement, (Fig. 5.3 (a)). However, that decrease was not uniform across the electron energy range. For instance, the difference in the decrease rate was visible on the average value of $B A S E 2$ and $W Z$ samples as a "dip" in the $S E Y$ curve in the energy range $E_{0}=450-1200 \mathrm{eV}$. For the $W Z$ sample, the highest rate of change in $S E Y$ was observed in the energy range $E_{0}=450-1200 \mathrm{eV}$. A decrease in $S E Y$, though at a lower rate, was 
also observed in energy range $E_{0}=60-450 \mathrm{eV}$. Last, there was little or no variation in $S E Y$ with successive measurements in the energy range $E_{0}=1200-2000 \mathrm{eV}$. On the other hand, even though a similar trend was observed in BASE 2 sample, the highest rate of SEY change was in energy range $E_{0}=60-400$, followed by the range $E_{0}=400-1000 \mathrm{eV}$. In the high energy range $E_{0}=1000$ $2000 \mathrm{eV}$ there was little or almost no variation in SEY magnitude. In contrast to BASE 2 and $W Z$ sample, $B A S E 1$ surface had a lower average $S E Y$ and minor variation of results without any definite trend in change. The surface of the BASE 1 can be considered as processed by the electron beam since it was used for calibration measurements.

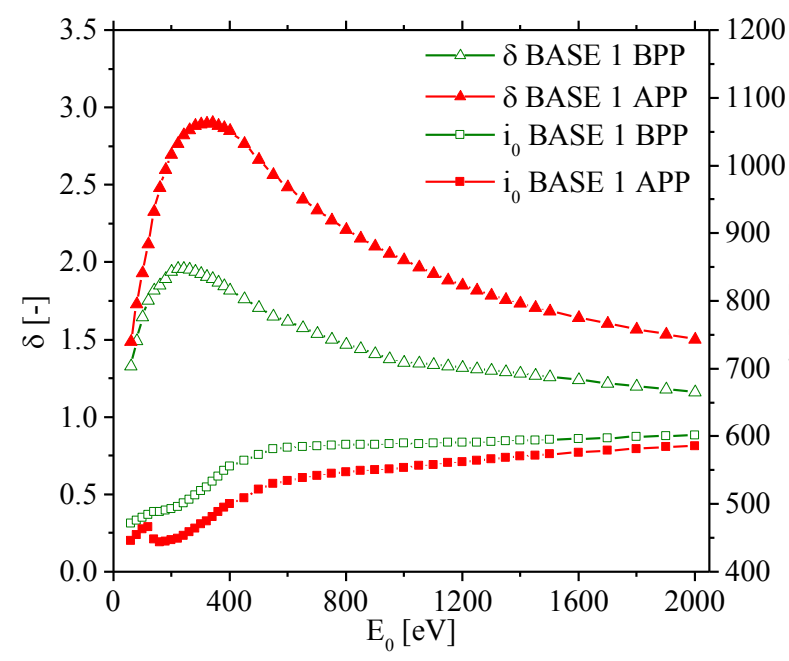

(a)

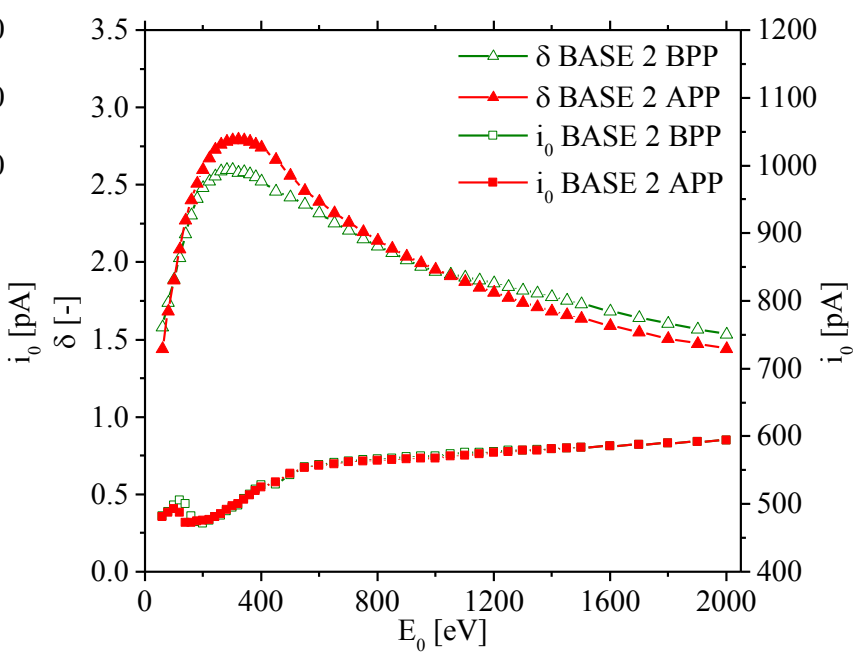

(b)

Fig. 5.4 SEY curves (triangle markers) and corresponding $i_{0}$ (square markers) for the first of seven measurements before $(B P P)$ and after $(A P P)$ nitrogen plasma processing as a function of $E_{0}$, for (a) $B A S E 1$ and (b) $B A S E 2$ samples. 


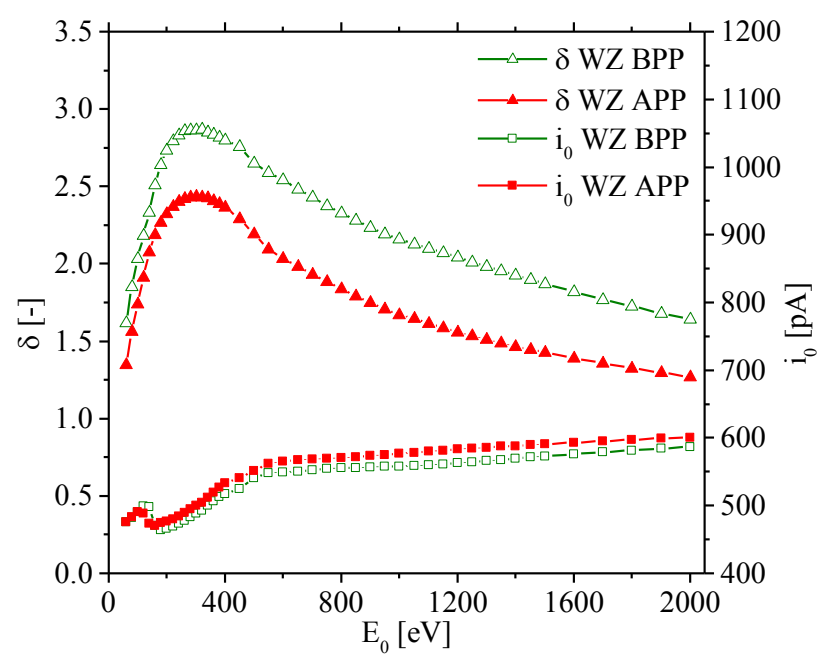

Fig. 5.5 SEY curves (triangle markers) and corresponding $i_{0}$ (square markers) for the first of seven measurements before $(B P P)$ and after $(A P P)$ nitrogen plasma processing as a function of $E_{0}$, for WZ sample.

The same experimental procedure was repeated after samples were processed with nitrogen plasma. A significant reduction in variation of $S E Y$ was observed as consequence of nitrogen plasma processing. It can be concluded that the nitrogen plasma modified the surface of the sample that now exhibits the increased resistance to repeated exposures of the electron beam. However, after plasma processing the $S E Y$ magnitudes of each sample gave different average values when compared to the results before plasma processing. The differences between the first of seven measurements of $S E Y$ and the corresponding primary electron beam current curves before $(B P P)$ and after $(A P P)$ plasma processing are shown in Fig. 5.4 andFig. 5.5. The $S E Y$ values of $B A S E 1$ sample increased along the entire range of primary electron energies after plasma processing. Before plasma processing, the maximum yield was $\delta_{\max }=1.96$ at $E_{0}=240$ $\mathrm{eV}$, while after plasma processing, the same parameters increased to $\delta_{\max }=2.90$ at $E_{0}=320 \mathrm{eV}$. However, the $S E Y$ curve of $B A S E 2$ did not change significantly in magnitudes across the energy range after plasma processing. There were two points where the $S E Y$ curve of sample after 
plasma processing traverses the $S E Y$ curve of the sample before plasma processing. The first intersecting point occurs at approximately $E_{0}=100 \mathrm{eV}$ where the $S E Y$ values after plasma processing were lower than before plasma processing. Above the primary electron energy of 100 $\mathrm{eV}$ and below $1050 \mathrm{eV}, S E Y$ was higher after plasma processing. The second intersecting point was measured at roughly $E_{0}=1050 \mathrm{eV}$. Above this energy, the $S E Y$ of sample after plasma processing was lower than $S E Y$ of samples before plasma processing. Specifically, the initial maximum yield at which this was occurring was $\delta_{\max }=2.60$, while primary electron energy was $E_{0}=280 \mathrm{eV}$. After plasma processing, these values increased to $\delta_{\max }=2.79$ at primary electron energy $E_{0}=320 \mathrm{eV}$. The primary electron beam currents on $B A S E 2$ were almost identical across the energy range indicating that the $S E Y$ change can be attributed to the effect of nitrogen plasma. In contrast to the BASE 1 and $B A S E 2$ samples, the $S E Y$ of the $W Z$ sample showed a reduction across the entire energy range of primary electrons after plasma processing. Before plasma processing, the maximum yield was $\delta_{\max }=2.87$ at $E_{0}=320 \mathrm{eV}$, after plasma processing it was reduced to $\delta_{\max }=2.44$ at $E_{0}=300 \mathrm{eV}$.

Seven measurements were performed on a single spot of each sample before and after plasma treatment. It was observed that nitrogen plasma processing reduced variation of $S E Y$. However, after plasma processing the average $S E Y$ increased on the samples representing the base metal. This increase may be caused by exposure of samples to air at atmospheric pressure during the transfer between the processing and $S E Y$ measurement chamber, as a similar effect was observed in [3].

\subsection{The $\mathrm{Ar} / \mathrm{O}_{2}$ plasma processing results}

In order to study the effects of $\mathrm{Ar} / \mathrm{O}_{2}$ plasma on $S E Y$ of niobium, three sets of nine samples were fabricated according to the description in section 4.2 (BASE, WELD, and OFFSET sample 
sets). On these three sample sets, four characteristic surfaces were selected to perform the $S E Y$ measurements. The SEY results were combined into four groups based on the characteristic surface where the measurements were performed. The first group of results was $B A S E$, representing the $S E Y$ measured on the base metal. The second group was $W Z$, representing the SEY measured on the weld zones of WELD and OFFSET sample sets. The third group was HAZ, representing the $S E Y$ measured on heat affected zones of WELD and OFFSET sample sets. Last, the fourth group of results was $R-H A Z$, representing the $S E Y$ measured on the surface adjacent to the heat affected zone of the OFFSET sample set. In addition, the effect of incident angle of primary electrons on $S E Y$ was measured by orienting the sample surfaces with respect to the primary electron beam.



Fig. 5.6 Average primary electron beam current $\left(i_{0}\right)$ and standard deviation as a function of $E_{0}$.

The total number of 378 SEY measurements was performed on the three sample sets. Specifically, half of that number was executed on samples before they were processed with plasma and the other half after the plasma processing. The variation of primary electron beam 
current amongst different measurements was less than $10 \%$. Since the variation of the primary electron beam was small individual primary electron beam curves for the $S E Y$ measurements are not included. Instead, an average value and standard deviation were calculated to represent the variation of the primary electron beam current (Fig. 5.6). The statistical bars covered approximately $\pm 24 \mathrm{pA}$ from average value at each energy level where the measurements were recorded.

It can be observed that the primary electron beam current curve has two distinct sections. The first section, in the energy range $E_{0}=60-400 \mathrm{eV}$ current had a steep steady rise from $350 \mathrm{pA}$ to $439 \mathrm{pA}$ on average. The second section, in the energy range $E_{0}=400-2000 \mathrm{eV}$ has a low current increase from $439 \mathrm{pA}$ to $462 \mathrm{pA}$ on average. Consequently, this adds to a total increase of $112 \mathrm{pA}$ in the primary electron beam current over the entire energy range. The cause of this instability can be traced to electron gun optics parameters that were set to maintain the size of the electron beam cross section (see Section 4.2.3). The difference in the primary electron beam current at the low energy range and at the high energy range did not significantly affect the results of the $S E Y$.

\subsubsection{SEY measured at $\theta=0^{\circ}$}

A sample from each set was positioned to form a $0^{\circ}$ incident angle (normal to the sample surface) with the primary electron beam. This sample position allowed for nine measurement spots on each sample surface. 


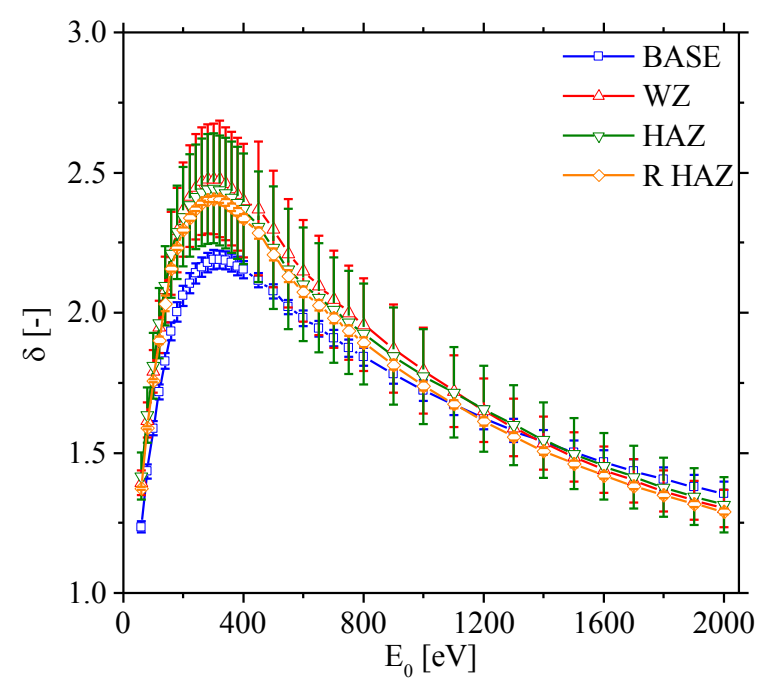

(a)

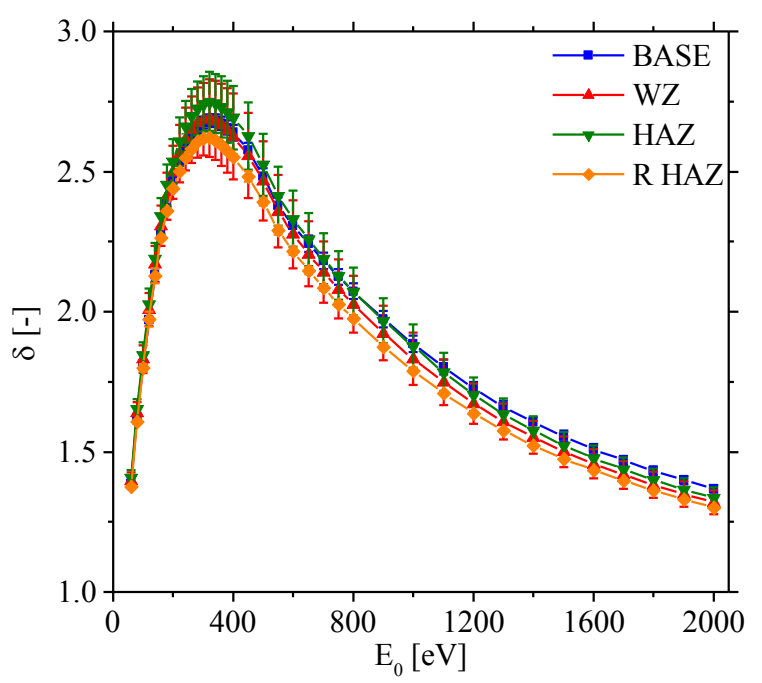

(b)

Fig. 5.7 The average $S E Y$ with sample standard deviation bars as a function of $E_{0}$ at $\theta=0^{\circ}$, (a) before and (b) after plasma processing.

Before plasma processing, the average $S E Y$ of $B A S E$ surface was lower when compared to the remaining characteristic surfaces for $E_{0}=60-1400 \mathrm{eV}$ (Fig. 5.7 (a)). The variation of the $S E Y$ across the surface of the $B A S E$ sample was no more than $3 \%$. The average $S E Y$ of the characteristic surfaces found in the weld area $(W Z, H A Z$, and $R-H A Z)$ are grouped together, which may be the consequence of heat created during the welding procedure, different microstructure or decreased number of grain boundaries. It was reported [17] that the $S E Y$ changes after heat treatment of the material. The variation of the $S E Y$ across the characteristic surfaces of the weld area was much larger when compared to the variation of the $B A S E$ surface. This may be due to the absorbed gases by surfaces of the weld area during cool-down after welding procedure.

After plasma processing, average $S E Y$ values have increased and have also grouped together at approximately the same value (Fig. 5.7 (b)). The variation of the $S E Y$ across the characteristic surfaces decreased. A comparable average $S E Y$ trend and reduction in variation 
indicate that the plasma processing formed the uniform $S E Y$ surface layer on all samples and characteristic surfaces. Increase in the average $S E Y$ may have occurred due to the surface reactivity with air at atmospheric pressure. A similar effect was described in [3], where the $S E Y$ of the plasma treated surface increased after air exposure. The maximum average $S E Y$ values of samples before and after plasma processing are given in Table 5.1. In addition, the possible range of the $S E Y$ values on the entire surface of the cavity was inferred based on the obtained results from the samples by calculating the $90 \%$ prediction interval for characteristic surfaces (Fig. 5.8). A reduction of the $S E Y$ variation after plasma processing led to a narrower prediction interval.



(a)

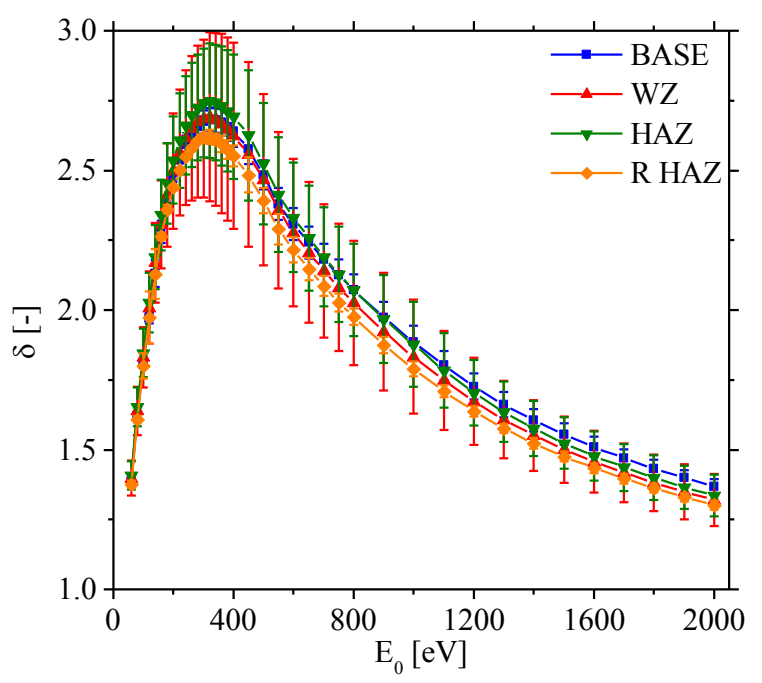

(b)

Fig. 5.8 The average $S E Y$ with $90 \%$ prediction interval bars as a function of $E_{0}$ at $\theta=0^{\circ}$, (a) before and (b) after plasma processing. 
Table 5.1 Average maximum yield $\left(\bar{\delta}_{\max }\right)$ and corresponding energy $\left(E_{0}\right)$, (a) before and (b) after plasma processing at $\theta=0^{\circ}$.

\begin{tabular}{ccccc}
\hline \hline $\begin{array}{c}\text { Characteristic } \\
\text { surface }\end{array}$ & \multicolumn{2}{c}{ (a) } & \multicolumn{2}{c}{ (b) } \\
\hline$B A S E$ & 2.19 & 320 & 2.68 & 320 \\
$W Z$ & 2.48 & 320 & 2.69 & 320 \\
$H A Z$ & 2.44 & 320 & 2.75 & 320 \\
$R-H A Z$ & 2.40 & 320 & 2.62 & 320 \\
\hline
\end{tabular}

\subsubsection{SEY measured at $\theta=+15^{\circ}$}

The $S E Y$ was measured at the $+15^{\circ}$ of the incident angle on a sample from each set. At this incident angle, there are nine measurement spots on a sample where $S E Y$ measurements were taken.

Before plasma processing, the average values of $S E Y$ were different between characteristic surfaces. The $S E Y$ curves of $W Z, H A Z$, and $R-H A Z$ surfaces have a similar general shape, though different magnitudes across the energy range of primary electrons (Fig. 5.9 (a)). The BASE surface had a different slope after $320 \mathrm{eV}$ of primary electron energy, when compared to the weld area surfaces. Variation of the $S E Y$ is the largest on the $W Z$ surface and reduces as the measurement spot moves farther from the weld zone. The smallest variation is measured on the $\mathrm{B} A S E$ surface. 


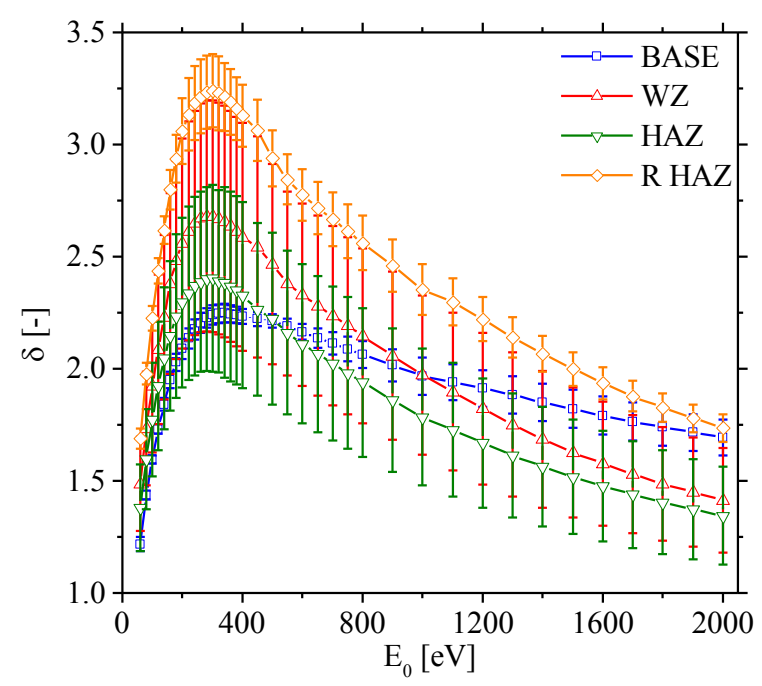

(a)



(b)

Fig. 5.9 The average $S E Y$ with sample standard deviation bars as a function of $E_{0}$ at $\theta=+15^{\circ}$, (a) before and (b) after plasma processing.

After plasma processing, average $S E Y$ values have approximately the same value for all three surfaces of the weld area $(W Z, H A Z$, and $R-H A Z)$. Almost an exact overlap was observed on the weld area characteristic surfaces (Fig. 5.9 (b)). The $S E Y$ of the $B A S E$ surface increased in the 60 to $1200 \mathrm{eV}$ energy range of the primary electrons and decreased in the 1200 to $2000 \mathrm{eV}$ energy range. The resulting $S E Y$ curve of the $B A S E$ shows better agreement with the $S E Y$ for the weld area characteristic surfaces. This indicates that regardless of the initial state of the surface, plasma processing resulted in a more uniform $S E Y$ properties of the surface layer.

Variability of the $S E Y$ magnitudes was significantly reduced on the $W Z, H A Z$ and $R-H A Z$ surfaces after plasma processing. In contrast, the variability of the $S E Y$ on the $B A S E$ surface increased over the entire energy range. The changes in the average value of the maximum $S E Y$ values are presented in Table 5.2. Average maximum $S E Y$ increased for $B A S E$ and $H A Z$ surfaces, decreased for $R-H A Z$ surface, and remained approximately the same on the $W Z$ surface. A $90 \%$ prediction interval of the entire cavity surface was inferred based on the nine measurements 
performed on the sample from each set, before and after plasma processing (Fig. 5.10). It can be concluded that the plasma processing improved the uniformity of the surface when the $S E Y$ magnitude is considered.

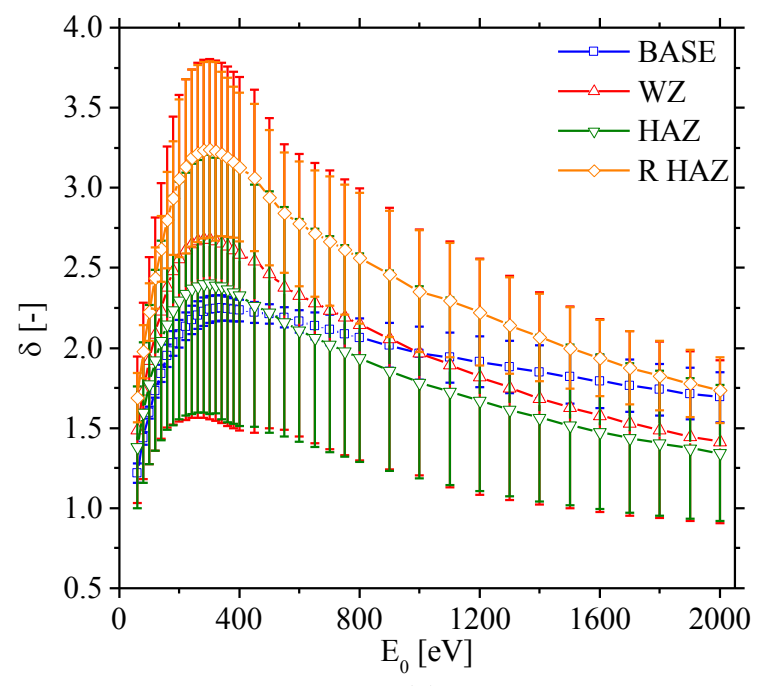

(a)

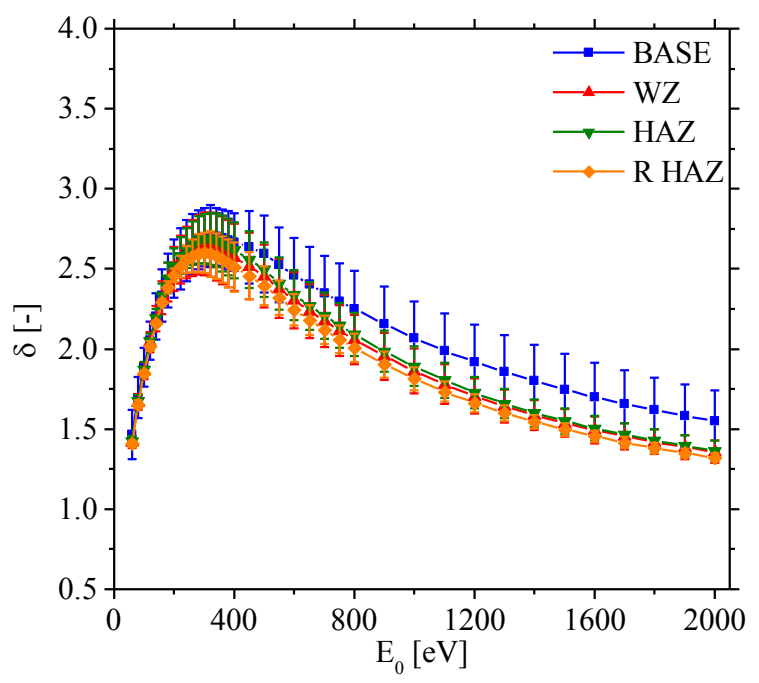

(b)

Fig. 5.10 The average $S E Y$ with $90 \%$ prediction interval bars as a function of $E_{0}$ at $\theta=+15^{\circ}$, (a) before and (b) after plasma processing.

Table 5.2 Average maximum yield $\left(\bar{\delta}_{\max }\right)$ and corresponding energy $\left(E_{0}\right)$, (a) before and (b) after plasma processing at $\theta=+15^{\circ}$.

\begin{tabular}{ccccc}
\hline \hline \multirow{2}{*}{$\begin{array}{c}\text { Characteristic } \\
\text { surface }\end{array}$} & \multicolumn{2}{c}{ (a) } & \multicolumn{2}{c}{ (b) } \\
\hline$B A S E$ & 2.25 & 320 & 2.71 & 320 \\
$W Z$ & 2.68 & 320 & 2.66 & 320 \\
$H A Z$ & 2.40 & 320 & 2.69 & 320 \\
$R-H A Z$ & 3.24 & 320 & 2.60 & 320 \\
\hline
\end{tabular}




\subsubsection{SEY measured at $\theta=-15^{\circ}$}

The examined sample was tilted to form a $-15^{\circ}$ incident angle with the primary electron beam. The $S E Y$ measurements were performed on one sample from each set. The measurements were made at nine different spots on each sample and were used to calculate the average, sample standard deviation, and $90 \%$ prediction interval spots on each sample were used to obtain the SEY results and calculate the average, sample standard deviation, and a $90 \%$ prediction interval.

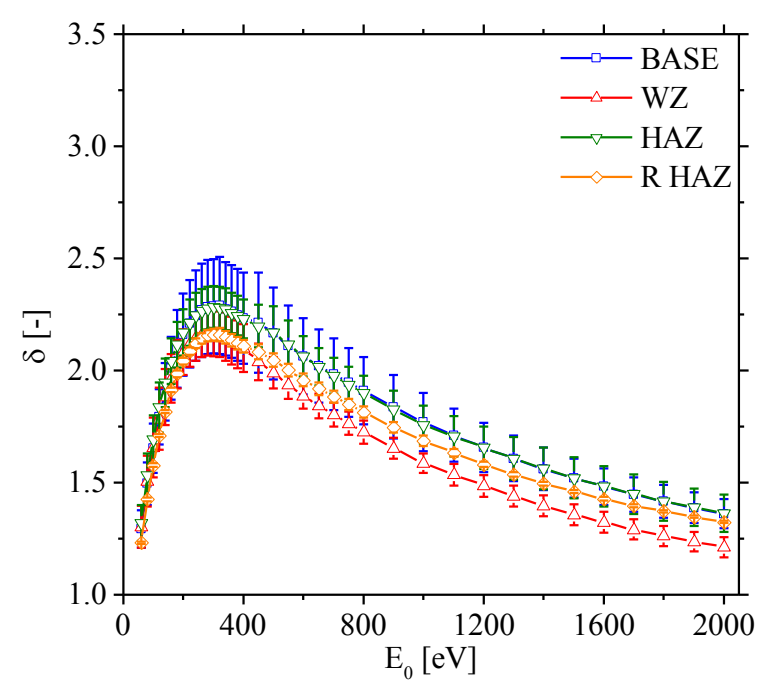

(a)

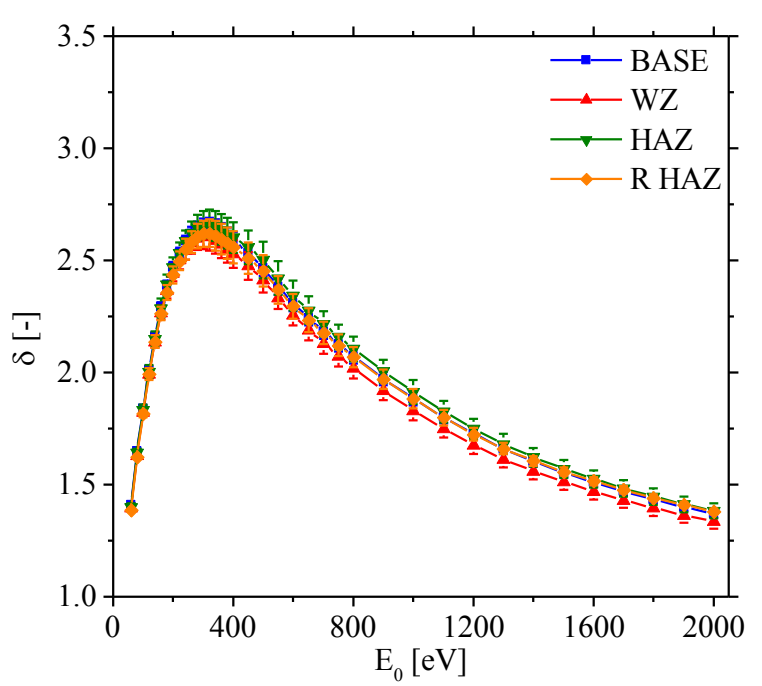

(b)

Fig. 5.11 The average $S E Y$ with sample standard deviation bars as a function of $E_{0}$ at $\theta=-15^{\circ}$, (a) before and (b) after plasma processing.

Before plasma processing, the $S E Y$ curves of all characteristic surfaces have the same general trend (Fig. 5.11 (a)). The BASE and $H A Z$ surface have the same average $S E Y$ values across the energy range and the same variation above $1200 \mathrm{eV}$ of primary electron energy. Below that energy, $B A S E$ surface exhibits larger $S E Y$ variation. The average $S E Y$ values for $W Z$ and $R-H A Z$ are the same up to $400 \mathrm{eV}$ of primary electron energy. Above that energy level, $R$ - 
$H A Z$ surface has a slightly higher $S E Y$ when compared to the $W Z$. The variation of the $S E Y$ of both surfaces was no more than $4 \%$.

Table 5.3 Average maximum yield $\left(\bar{\delta}_{\max }\right)$ and corresponding energy $\left(E_{0}\right)$, (a) before and (b) after plasma processing at $\theta=-15^{\circ}$.

\begin{tabular}{ccccc}
\hline \hline Characteristic & \multicolumn{2}{c}{ (a) } & \multicolumn{2}{c}{ (b) } \\
surface & $\bar{\delta}_{\max }[-]$ & $E_{0}[\mathrm{eV}]$ & $\bar{\delta}_{\max }[-]$ & $E_{0}[\mathrm{eV}]$ \\
\hline$B A S E$ & 2.29 & 320 & 2.66 & 320 \\
$W Z$ & 2.17 & 320 & 2.61 & 320 \\
$H A Z$ & 2.28 & 320 & 2.66 & 320 \\
$R-H A Z$ & 2.16 & 320 & 2.62 & 320 \\
\hline
\end{tabular}

After plasma processing, the average SEY magnitudes of all characteristic surfaces increased to approximately the same value (Fig. 5.11 (b)). Variation of the SEY results of all surfaces decreased, except for $R-H A Z$ surface which exhibited a minor increase in variability. The change in magnitude of the average maximum $S E Y$ for characteristic surfaces is given in Table 5.3. In addition, the predicted $S E Y$ values on the entire surface of the cavity was inferred by calculating the $90 \%$ prediction interval (Fig. 5.12). The increase in variability of $S E Y$ results on $R-H A Z$ surface caused the increase of the range of possible $S E Y$ values after plasma processing. 


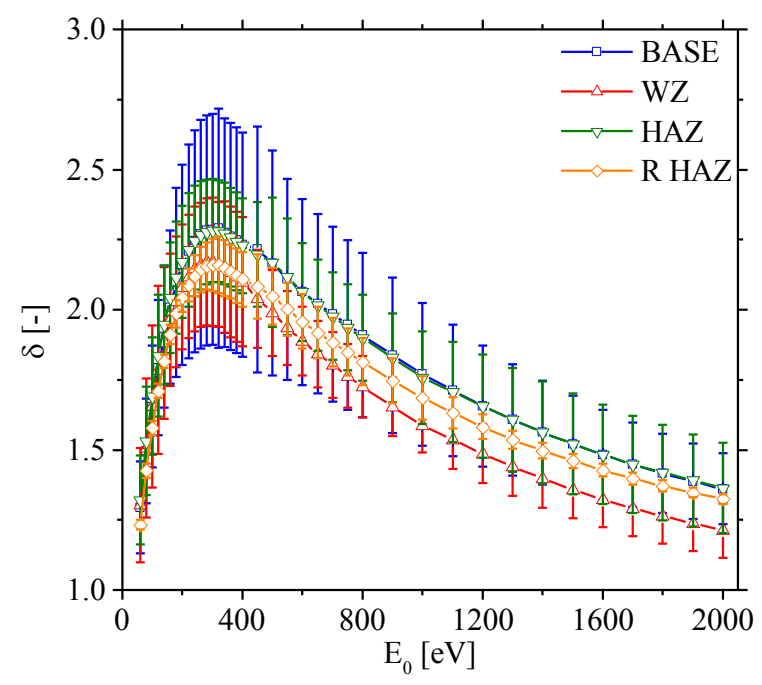

(a)

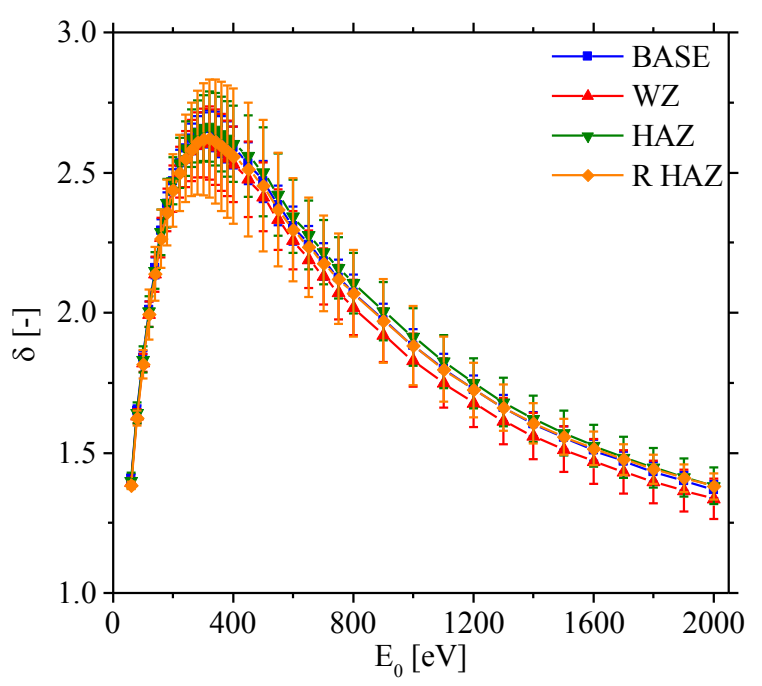

(b)

Fig. 5.12 The average $S E Y$ with $90 \%$ prediction interval bars as a function of $E_{0}$ at $\theta=-15^{\circ}$, (a) before and (b) after plasma processing.

\subsubsection{SEY measured at $\theta=+30^{\circ}$}

A sample from each set was tilted so that the normal to the surface formed $+30^{\circ}$ angle with the primary electron beam. At $+30^{\circ}$ angle, $S E Y$ measurements were made at nine different measurement spots.

Before plasma processing, the average $S E Y$ values can be separated in two groups (Fig. 5.13 (a)). In the first group, $B A S E$ and $H A Z$ surfaces had almost the same average $S E Y$ values across the energy range of primary electrons. Variation of the $S E Y$ measurements for these surfaced were no more than $8 \%$ and approximately the same. In the second group, $W Z$ and $R$ $H A Z$ surfaces had almost the same average $S E Y$ values. However, variation of the $S E Y$ measurements was more significant on the $W Z$ surface. Variation of the $S E Y$ measurements on $R$ $H A Z$ was comparable to $B A S E$ and $H A Z$ surfaces. 


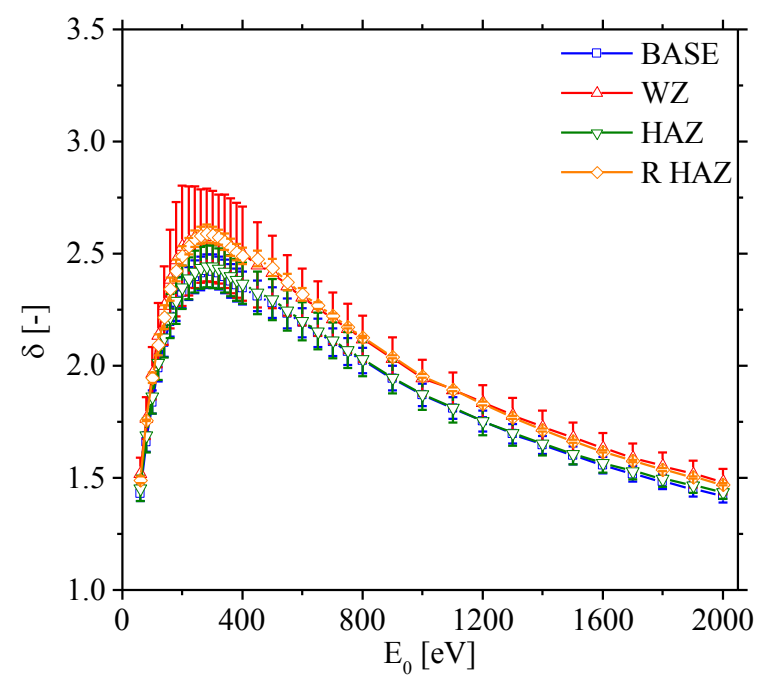

(a)

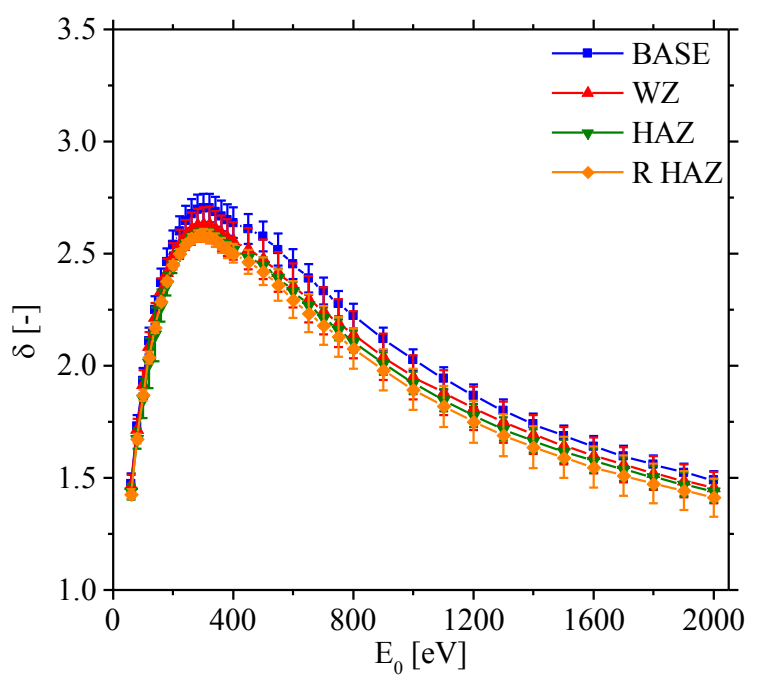

(b)

Fig. 5.13 The average $S E Y$ with sample standard deviation bars as a function of $E_{0}$ at $\theta=+30^{\circ}$, (a) before and (b) after plasma processing.

Table 5.4 Average maximum yield $\left(\bar{\delta}_{\max }\right)$ and corresponding energy $\left(E_{0}\right)$, (a) before and (b) after plasma processing at $\theta=+30^{\circ}$.

\begin{tabular}{ccccc}
\hline \hline $\begin{array}{c}\text { Characteristic } \\
\text { surface }\end{array}$ & \multicolumn{2}{c}{ (a) } & \multicolumn{2}{c}{ (b) } \\
\hline$B A S E$ & 2.42 & 320 & 2.70 & 320 \\
$W Z$ & 2.58 & 320 & 2.63 & 320 \\
$H A Z$ & 2.44 & 320 & 2.59 & 320 \\
$R-H A Z$ & 2.58 & 320 & 2.58 & 320 \\
\hline
\end{tabular}

After plasma processing, average $S E Y$ values increased for all characteristic surfaces (Fig. 5.13 (b)). The average $S E Y$ on the $B A S E$ surface increased more when compared to the surfaces of the weld area. The variation of the $S E Y$ measurements was reduced for the $H A Z$ surface, and remained approximately the same $(2 \%)$ on the $B A S E$ surface. The $W Z$ surface exhibited a reduction in $S E Y$ measurements variation over the entire energy range of the primary electrons. The variation of the $S E Y$ results on the $R-H A Z$ surface remained the same for primary electron 
energies up to $400 \mathrm{eV}$, after which there was an increase. The changes in the average maximum SEY values of the characteristic surfaces are given in Table 5.4. The $90 \%$ prediction interval of the entire cavity surface was inferred based on the nine measurements performed on the sample from each set, before and after plasma processing (Fig. 5.14).

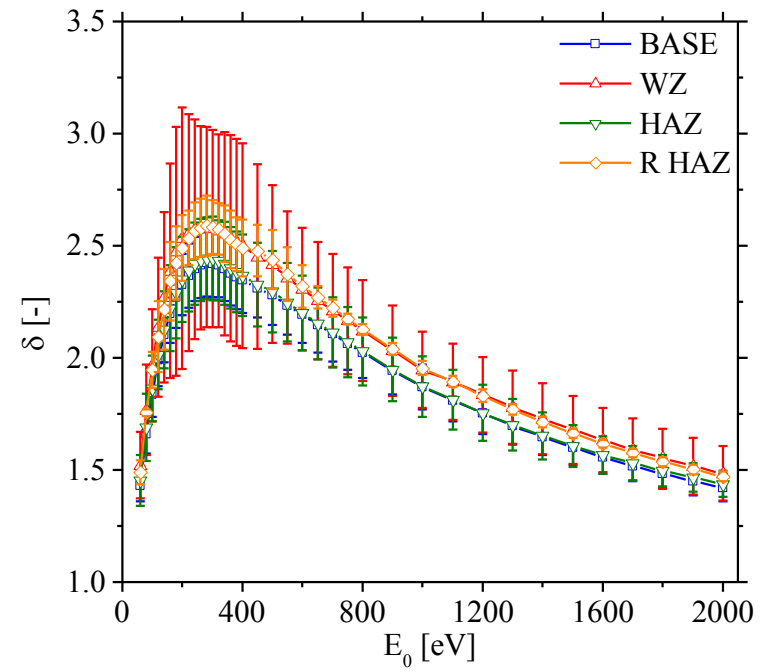

(a)

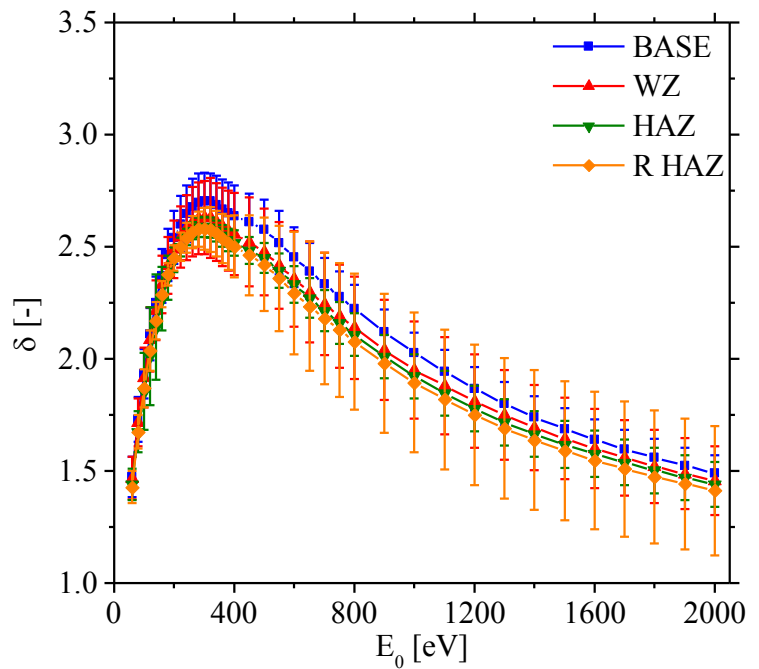

(b)

Fig. 5.14 The average $S E Y$ with $90 \%$ prediction interval bars as a function of $E_{0}$ at $\theta=+30^{\circ}$, (a) before and (b) after plasma processing.

\subsubsection{SEY measured at $\theta=-30^{\circ}$}

A sample from each set was used to measure the $S E Y$ at $-30^{\circ}$ incident angle of primary electrons. The SEY measurements were made at nine different measurement spots on each sample. 




(a)

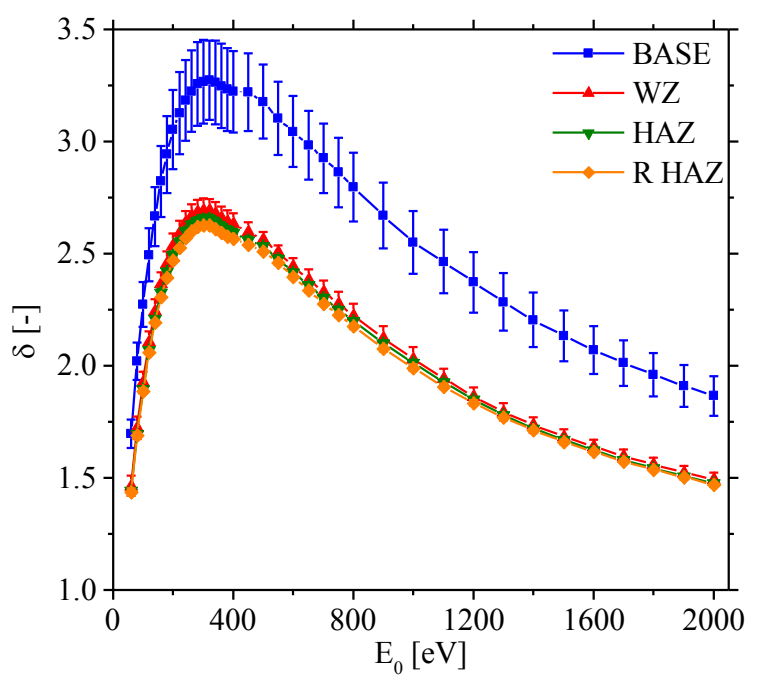

(b)

Fig. 5.15 The average $S E Y$ with sample standard deviation bars as a function of $E_{0}$ at $\theta=-30^{\circ}$, (a) before and (b) after plasma processing.

Before plasma processing, the average maximum $S E Y$ values were in the range from 2.25 to 2.40, and $S E Y$ curves followed the same trend (Fig. 5.15 (a)). The variation of the $S E Y$ results is the largest for $B A S E$ surface, followed by $W Z, H A Z$, and $R-H A Z$.

After plasma processing, all characteristic surfaces exhibit the increase in average $S E Y$ (Fig. 5.15 (b)). However, the $B A S E$ surface increase was significantly higher when compared to the weld area surfaces. Variation of the $S E Y$ measurements on the $B A S E$ surface were marginally reduced. The $S E Y$ of the weld area surfaces have converged to the same average values, while variation of the $S E Y$ magnitudes has decreased significantly after plasma processing. The changes in the average maximum $S E Y$ on the characteristic surface are presented in the Table 5.5. Based on the $S E Y$ measurements performed on the sample, range of yield magnitudes of the characteristic surfaces of the cavity was inferred by calculating the $90 \%$ prediction interval (Fig. 5.16). 
Table 5.5 Average maximum yield $\left(\bar{\delta}_{\max }\right)$ and corresponding energy $\left(E_{0}\right)$, (a) before and (b) after plasma processing at $\theta=-30^{\circ}$.

\begin{tabular}{ccccc}
\hline \hline Characteristic & \multicolumn{2}{c}{ (a) } & \multicolumn{2}{c}{ (b) } \\
surface & $\bar{\delta}_{\max }[-]$ & $E_{0}[\mathrm{eV}]$ & $\bar{\delta}_{\max }[-]$ & $E_{0}[\mathrm{eV}]$ \\
\hline$B A S E$ & 2.41 & 320 & 3.27 & 320 \\
$W Z$ & 2.49 & 320 & 2.69 & 320 \\
$H A Z$ & 2.36 & 320 & 2.66 & 320 \\
$R-H A Z$ & 2.25 & 320 & 2.63 & 320 \\
\hline
\end{tabular}

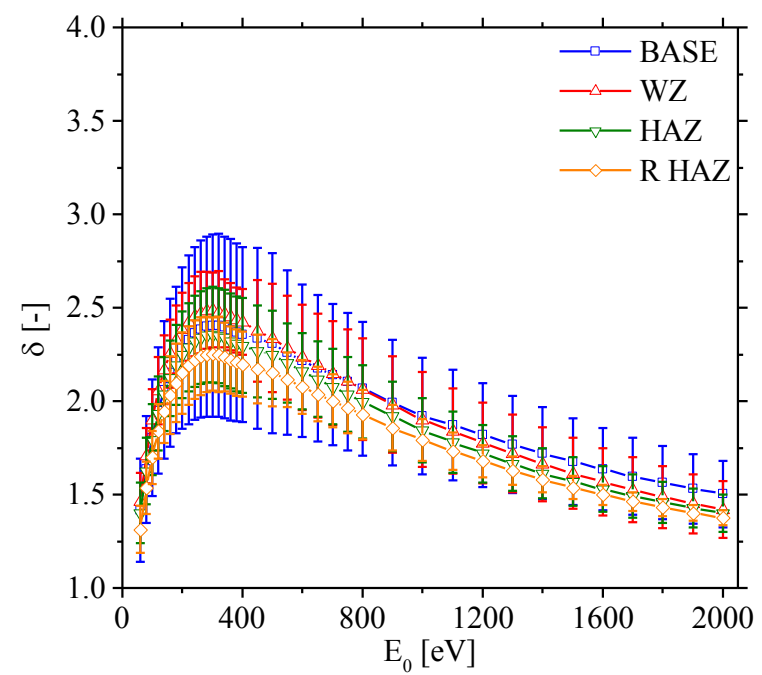

(a)

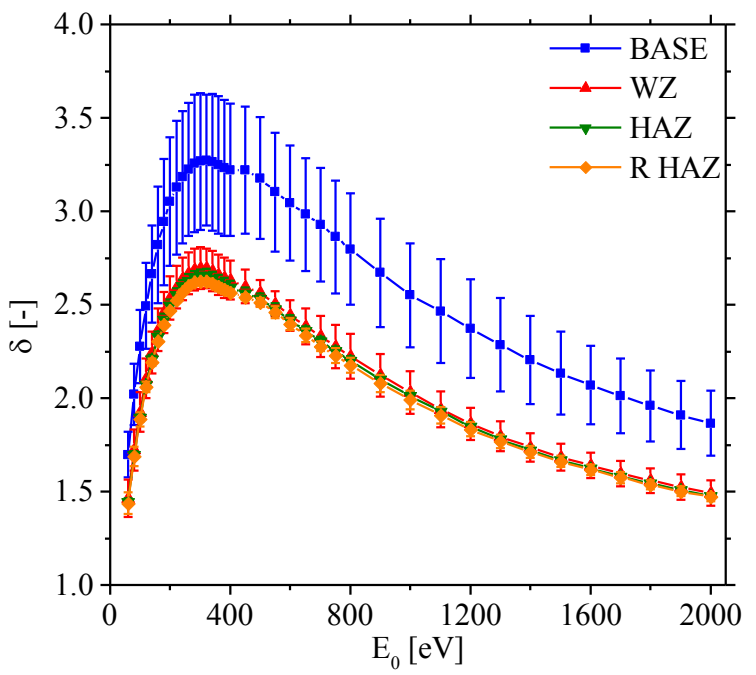

(b)

Fig. 5.16 The average $S E Y$ with $90 \%$ prediction interval bars as a function of $E_{0}$ at $\theta=-30^{\circ}$, (a) before and (b) after plasma processing.

\subsubsection{SEY measured at $\theta=+45^{\circ}$}

A sample from each set was tilted to form a $+45^{\circ}$ angle with the surface of the sample. Six measurement spots on each sample were used to determine distribution of the $S E Y$ across the weld area and base metal surfaces. 




(a)

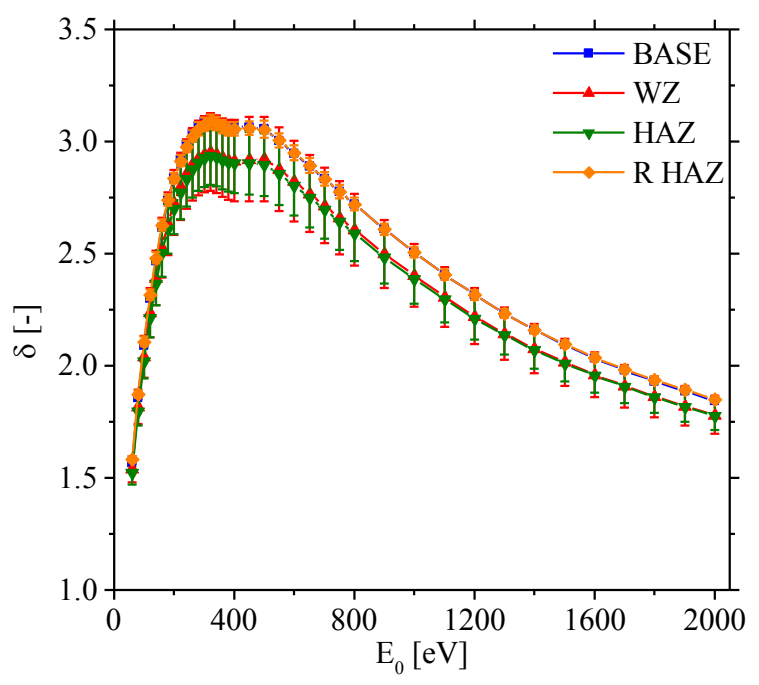

(b)

Fig. 5.17 The average $S E Y$ with sample standard deviation bars as a function of $E_{0}$ at $\theta=+45^{\circ}$, (a) before and (b) after plasma processing.

Before plasma processing, the highest average value of $S E Y$ was measured on the $B A S E$ surface, while the lowest was measured on $H A Z$ surface (Fig. 5.17 (a)). The $W Z$ and $R-H A Z$ had approximately the same average value of $S E Y$. A similar trend of the $S E Y$ curve was observed on all characteristic surfaces. The variation of the $S E Y$ results was the most prominent on $R-H A Z$ surface, followed by $B A S E, W Z$, and $H A Z$.

Table 5.6 Average maximum yield $\left(\bar{\delta}_{\max }\right)$ and corresponding energy $\left(E_{0}\right)$, (a) before and (b) after plasma processing at $\theta=+45^{\circ}$.

\begin{tabular}{ccccc}
\hline \hline Characteristic & \multicolumn{2}{c}{ (a) } & \multicolumn{2}{c}{ (b) } \\
surface & $\bar{\delta}_{\max }[-]$ & $E_{0}[\mathrm{eV}]$ & $\bar{\delta}_{\max }[-]$ & $E_{0}[\mathrm{eV}]$ \\
\hline$B A S E$ & 2.94 & 320 & 3.10 & 320 \\
$W Z$ & 2.68 & 320 & 2.95 & 320 \\
$H A Z$ & 2.53 & 320 & 2.94 & 320 \\
$R-H A Z$ & 2.65 & 320 & 3.09 & 320 \\
\hline
\end{tabular}




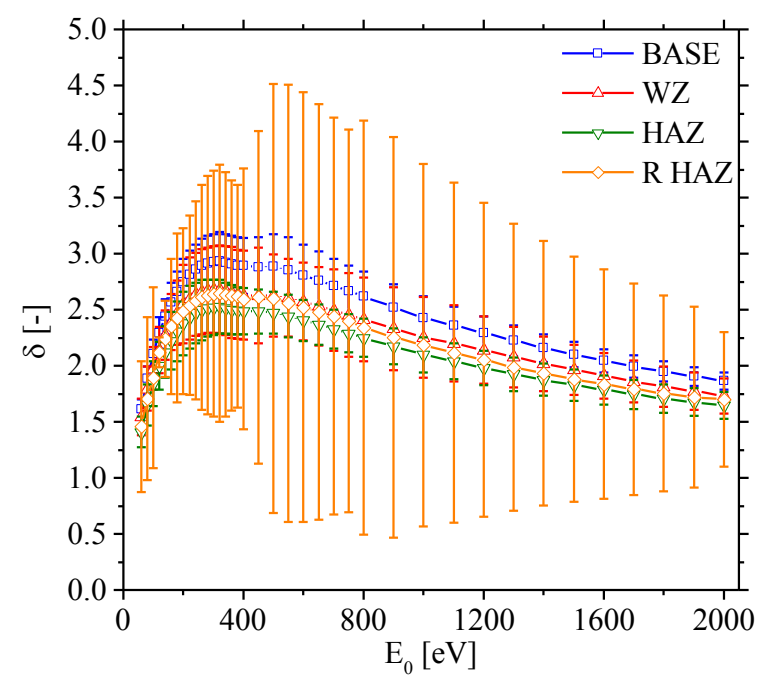

(a)

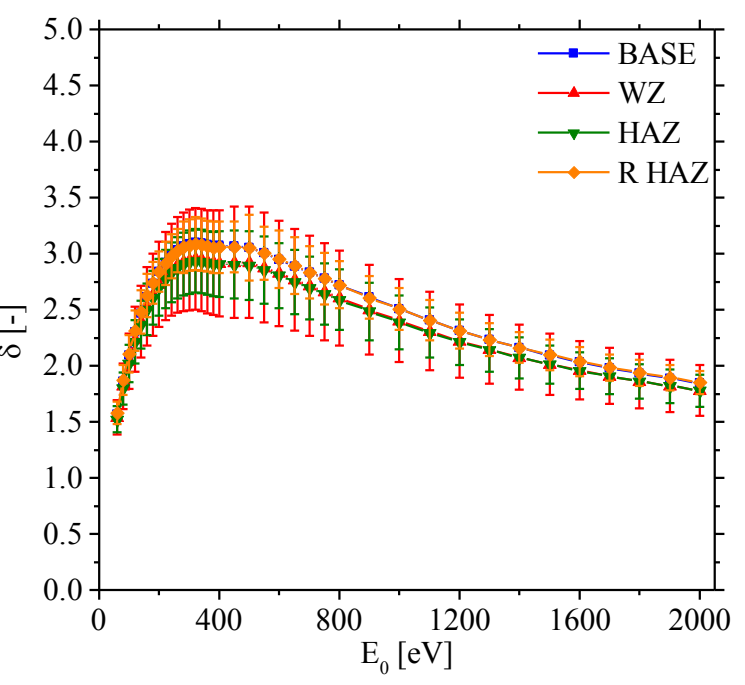

(b)

Fig. 5.18 The average $S E Y$ with $90 \%$ prediction interval bars as a function of $E_{0}$ at $\theta=+45^{\circ}$, (a) before and (b) after plasma processing.

After plasma processing, all characteristic surfaces exhibited the increase in average $S E Y$ values (Fig. 5.17 (b)). Differences in $S E Y$ magnitudes over the entire primary electron energy range were much smaller between different surfaces after plasma processing. The average maximum $S E Y$ values of characteristic surfaces before and after plasma processing are given in Table 5.6. Disparate changes in variation of the $S E Y$ measurements on characteristic surfaces were observed. A reduction in variation was observed on the BASE and $R-H A Z$ surfaces. The variation of the $S E Y$ results remained approximately the same for the $W Z$ and $H A Z$ surfaces. The 90\% prediction interval was calculated for all characteristic surfaces (Fig. 5.18). Since only two measurement spots were available for the $R-H A Z$ surface, the correction factor used to calculate the interval caused the inflation of the prediction interval. However, the same approach for calculating the prediction interval was used for the $S E Y$ results after plasma processing and much a narrower prediction interval was obtained. Taking the $R-H A Z$ surface as an example, improvement in uniformity of the $S E Y$ of a surface layer after plasma processing is evident. 


\subsubsection{SEY measured at $\theta=-45^{\circ}$}

The SEY measurements were performed on a sample from each set. Surfaces of the examined samples were tilted to form a $-45^{\circ}$ incident angle with the primary electron beam. At this incident angle, six measurement spots were available per sample.

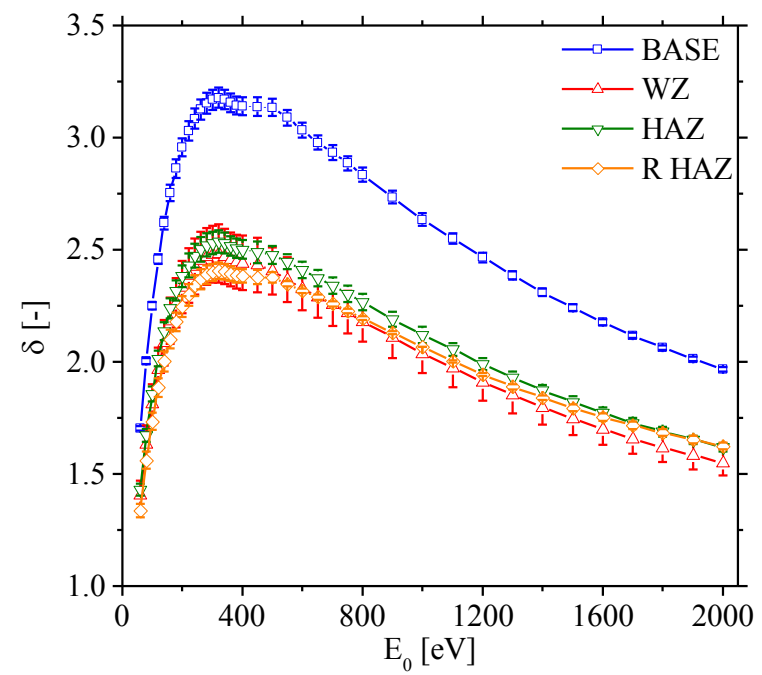

(a)

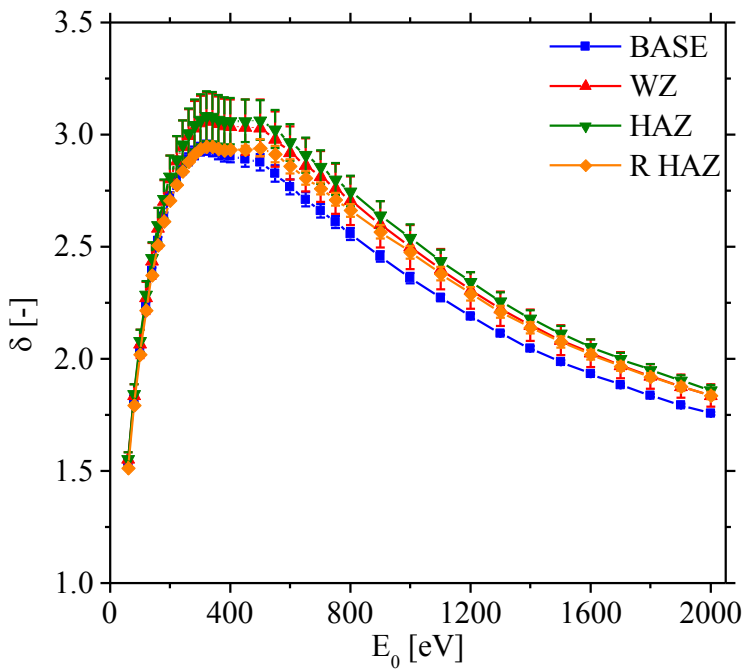

(b)

Fig. 5.19 The average $S E Y$ with sample standard deviation bars as a function of $E_{0}$ at $\theta=-45^{\circ}$, (a) before and (b) after plasma processing.

The $S E Y$ curves for samples before plasma processing are shown in Fig. 5.19 (a). The average $S E Y$ curves for the weld area characteristic surfaces were grouped together. It can be observed that there is less than $10 \%$ difference between the $S E Y$ values for the same energy level of primary electrons. On the other hand, the average $S E Y$ curve of $B A S E$ surface had much larger values compared to the weld area surfaces. The $10 \%$ variation of the $S E Y$ measurements was observed on the $W Z$ surface, whereas $S E Y$ variation was less than $4 \%$ for other surfaces. 
Table 5.7 Average maximum yield $\left(\bar{\delta}_{\max }\right)$ and corresponding energy $\left(E_{0}\right)$, (a) before and (b) after plasma processing at $\theta=-45^{\circ}$.

\begin{tabular}{ccccc}
\hline \hline $\begin{array}{c}\text { Characteristic } \\
\text { surface }\end{array}$ & \multicolumn{2}{c}{ (a) } & \multicolumn{2}{c}{ (b) } \\
\hline$B A S E$ & 3.18 & 320 & 2.94 & 320 \\
$W Z$ & 2.48 & 320 & 3.07 & 320 \\
$H A Z$ & 2.54 & 320 & 3.08 & 320 \\
$R-H A Z$ & 2.41 & 320 & 2.95 & 320 \\
\hline
\end{tabular}

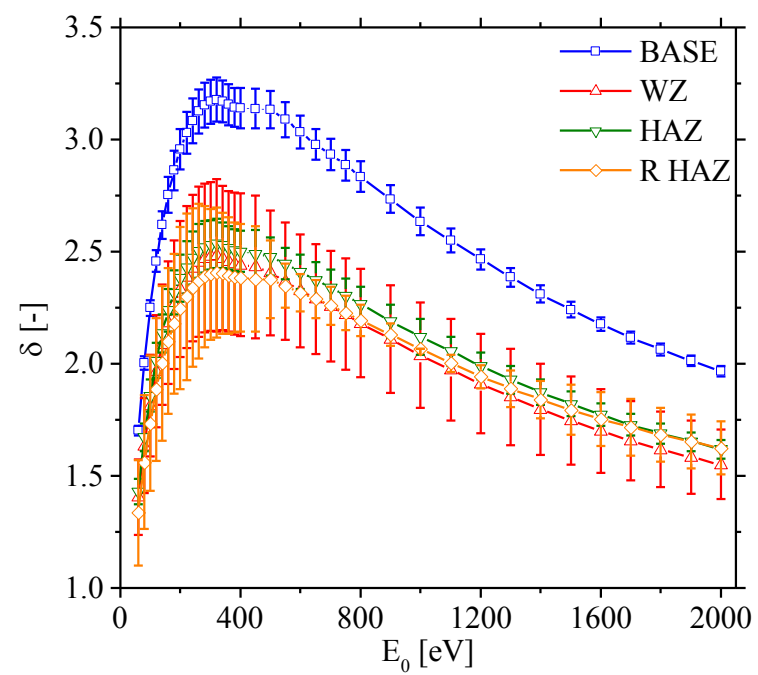

(a)

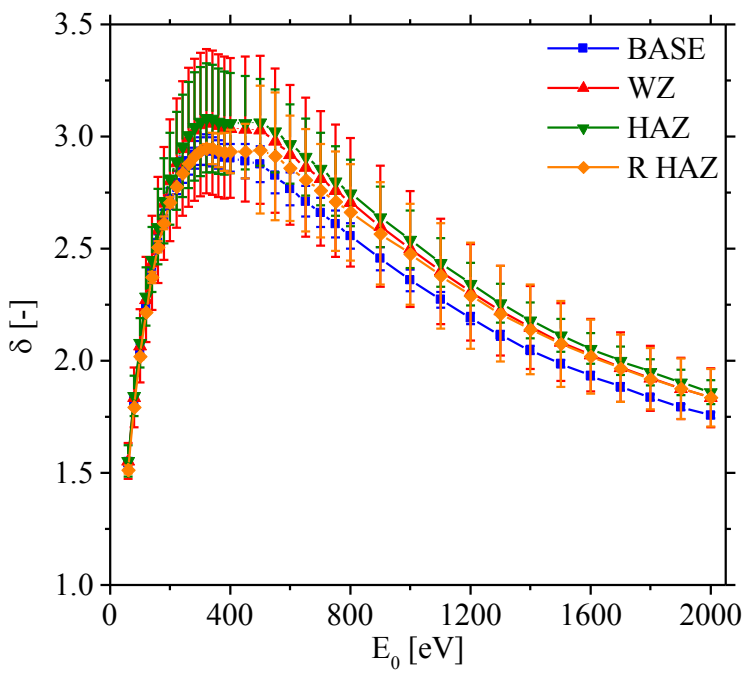

(b)

Fig. 5.20 The average $S E Y$ with $90 \%$ prediction interval bars as a function of $E_{0}$ at $\theta=-45^{\circ}$, (a) before and (b) after plasma processing.

After plasma processing, average $S E Y$ curves of characteristic surfaces have grouped together (Fig. 5.19 (b)). Reduction of the average $S E Y$ values can be observed on the $B A S E$ surface, while the average $S E Y$ curves of weld area surfaces have increased. The average maximum $S E Y$ values before and after plasma processing are given in Table 5.7. The variation of the $S E Y$ results was reduced on the $B A S E$ surface and the $R-H A Z$ surface up to $500 \mathrm{eV}$ of the primary electron energy, while the variation remained the same on the $W Z$ surface. The increase 
in the variation was observed on the $H A Z$ surface and the $R-H A Z$ surface for primary electron energies higher than $500 \mathrm{eV}$. Based on the measurements performed on the characteristic surfaces of the samples, 90\% prediction intervals are shown in Fig. 5.20.

\subsubsection{SEY measured at $\theta=+60^{\circ}$}

A sample from each set was used to measure the $S E Y$ at $+60^{\circ}$ incident angle of primary electrons, before and after plasma processing. At this incident angle, three measurement spots were available per sample.

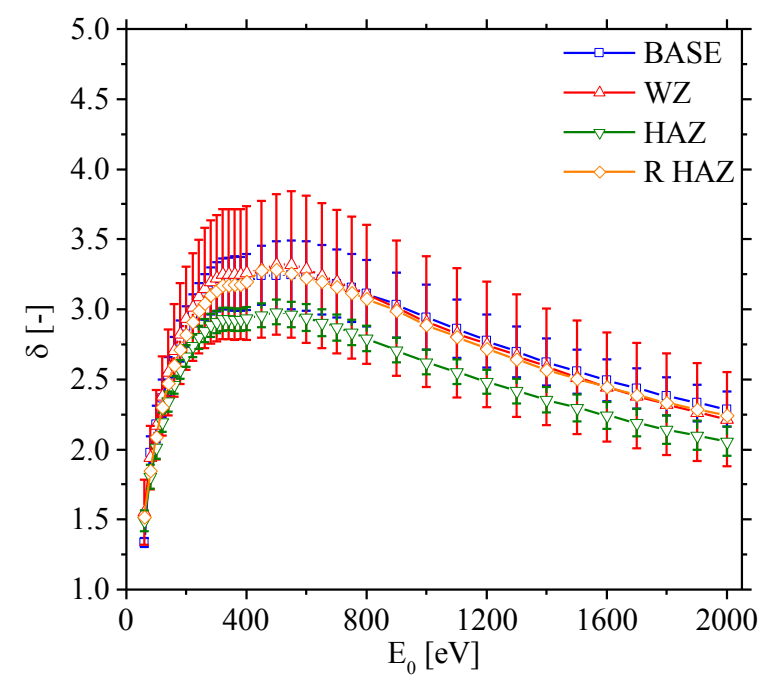

(a)



(b)

Fig. 5.21 The average $S E Y$ with sample standard deviation bars as a function of $E_{0}$ at $\theta=+60^{\circ}$, (a) before and (b) after plasma processing.

Before plasma processing, the average $S E Y$ curves follow the same trend. BASE, $W Z$ and $R$ $H A Z$ surfaces have an almost overlapping average $S E Y$, while the $H A Z$ surface has somewhat lower average $S E Y$ values (Fig. 5.21 (a)). The largest variation of $S E Y$ was observed on the $W Z$ 
surface, while the lowest was observed on the $H A Z$ surface. There are no standard deviation bars on $R-H A Z$ surface because only one measurement was performed on that characteristic surface.

After plasma processing, the average $S E Y$ values have increased for all characteristic surfaces (Fig. 5.21 (b)). The average $S E Y$ values of the $W Z$ and the $H A Z$ surfaces have completely overlapped. A somewhat higher average $S E Y$ was observed on the BASE and the $R$ $H A Z$ surfaces. The change between the average maximum $S E Y$ of the characteristic surfaces before and after plasma is given in Table 5.8. The variation of the $S E Y$ has decreased only on the $W Z$ and $B A S E$ surfaces. A small increase in $S E Y$ variation was observed on the $H A Z$ surface. The variation of $S E Y$ after plasma processing was almost the same for $W Z$ and $H A Z$ surfaces. The prediction interval for $W Z$ is fairly large, with $S E Y$ values going below zero, which is impossible (Fig. 5.22). This is due to the fact that only two measurements were performed at the $W Z$ surface. A large correction factor for small number of measurements inflated the prediction interval significantly. For the same surface, the prediction interval reduced significantly after plasma processing, indicating a more uniform surface layer, in terms of SEY.

Table 5.8 Average maximum yield $\left(\bar{\delta}_{\max }\right)$ and corresponding energy $\left(E_{0}\right)$, (a) before and (b) after plasma processing at $\theta=+60^{\circ}$.

\begin{tabular}{ccccc}
\hline \hline Characteristic & \multicolumn{2}{c}{ (a) } & \multicolumn{2}{c}{$(\mathrm{b})$} \\
surface & $\bar{\delta}_{\max }[-]$ & $E_{0}[\mathrm{eV}]$ & $\bar{\delta}_{\max }[-]$ & $E_{0}[\mathrm{eV}]$ \\
\hline$B A S E$ & 3.24 & 500 & 3.64 & 500 \\
$W Z$ & 3.32 & 500 & 3.49 & 500 \\
$H A Z$ & 2.98 & 500 & 3.48 & 500 \\
$R-H A Z$ & 2.28 & 500 & 3.72 & 500 \\
\hline
\end{tabular}




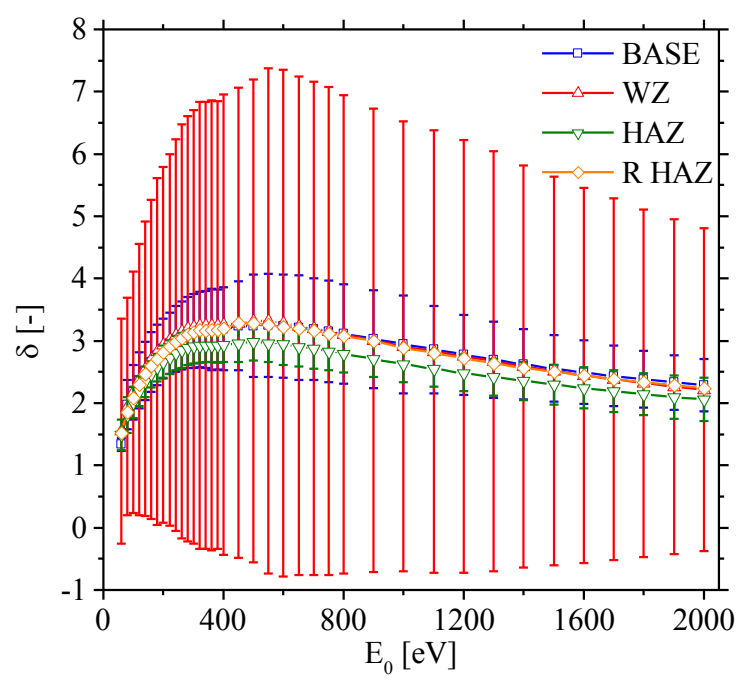

(a)

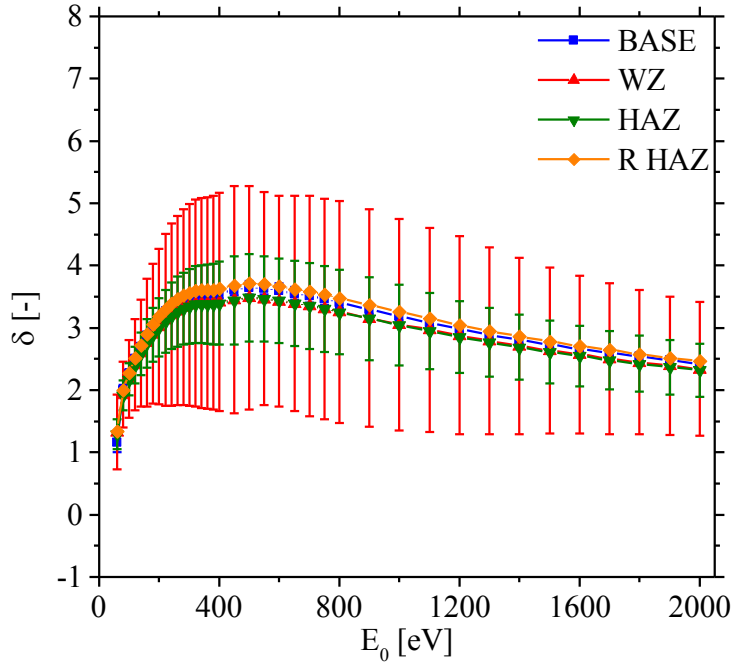

(b)

Fig. 5.22 The average $S E Y$ with $90 \%$ prediction interval bars as a function of $E_{0}$ at $\theta=+60^{\circ}$, (a) before and (b) after plasma processing.

\subsubsection{SEY measured at $\theta=-60^{\circ}$}

A sample from each set was positioned at a $-60^{\circ}$ incident angle with respect to the primary electron beam. Due to sample positioning constraints, only three measurement spots per sample were available.

Before plasma processing, the difference between the average $S E Y$ values of the $B A S E, W Z$ and $H A Z$, and $R-H A Z$ surfaces was significant (Fig. 5.23 (a)). The $S E Y$ measured at the $B A S E$ surface was the highest amongst all characteristic surfaces, and had the maximum variation less than $10 \%$. The $W Z$ and $H A Z$ surfaces had almost identical average $S E Y$ curves, while the variation of $S E Y$ measurements on each surface was comparable. No statistical calculation was made for the $R-H A Z$ surface, since only one measurement was performed due to limitation in sample positioning. 


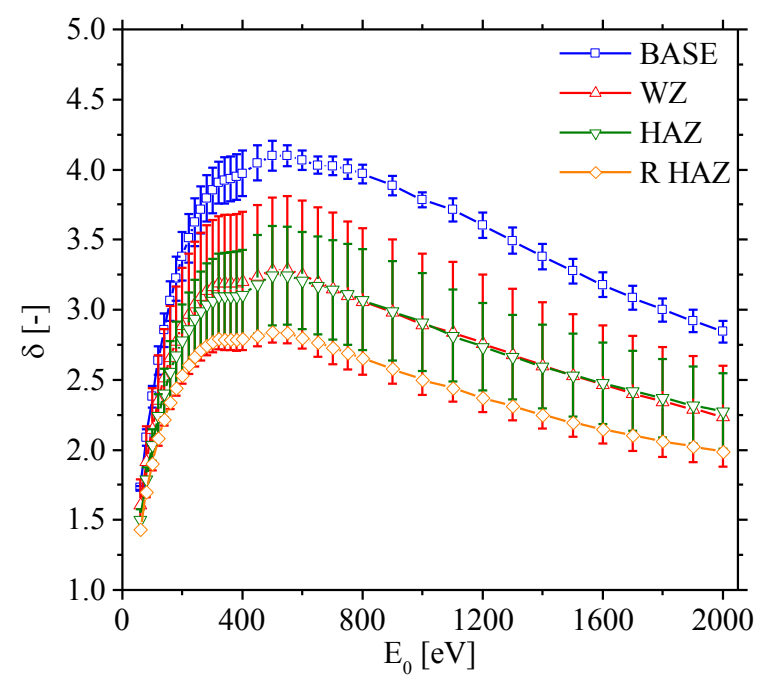

(a)

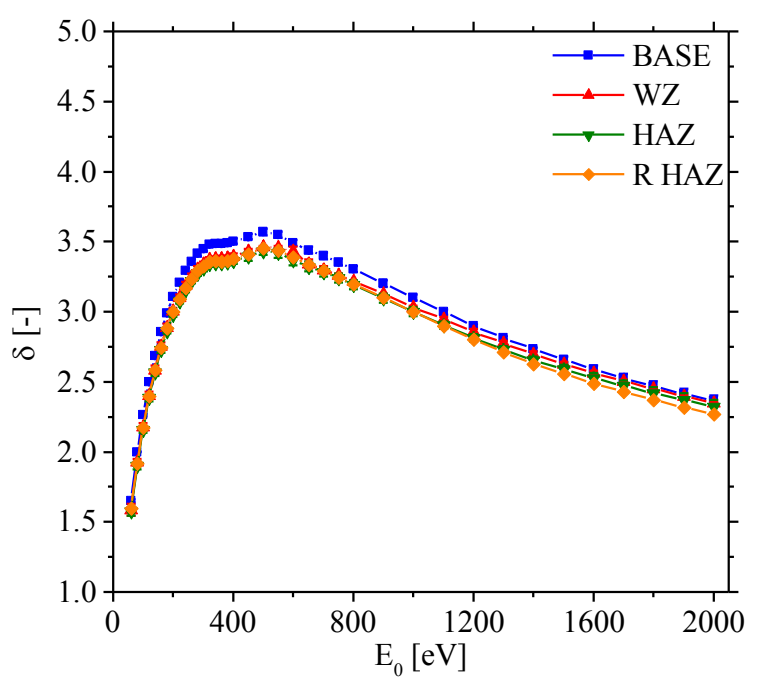

(b)

Fig. 5.23 The average $S E Y$ with sample standard deviation bars as a function of $E_{0}$ at $\theta=-60^{\circ}$, (a) before and (b) after plasma processing.

Table 5.9 Average maximum yield $\left(\bar{\delta}_{\max }\right)$ and corresponding energy $\left(E_{0}\right)$, (a) before and (b) after plasma processing at $\theta=-60^{\circ}$.

\begin{tabular}{ccccc}
\hline \hline $\begin{array}{c}\text { Characteristic } \\
\text { surface }\end{array}$ & \multicolumn{2}{c}{ (a) } & \multicolumn{2}{c}{ (b) } \\
\hline$B A S E$ & 4.10 & 500 & 3.57 & 500 \\
$W Z$ & 3.29 & 500 & 3.47 & 500 \\
$H A Z$ & 2.24 & 500 & 3.43 & 500 \\
$R-H A Z$ & 2.84 & 500 & 3.45 & 500 \\
\hline
\end{tabular}

After plasma processing, the average $S E Y$ curves of the characteristic surfaces have converged (Fig. 5.23 (b)). The average $S E Y$ values of weld area surfaces have increased across the entire energy range of primary electrons, whereas $S E Y$ values decreased for the $B A S E$ surface. The change of the average maximum $S E Y$ for all characteristic surfaces before and after plasma processing is given in Table 5.9. The variation of $S E Y$ results for the $B A S E, W Z$, and $H A Z$ surfaces was significantly reduced, except for the $R-H A Z$ surface which was determined for one 
spot only. The prediction interval of $90 \%$ was calculated for all characteristic surfaces except for the $R$-HAZ (Fig. 5.24). Due to the small number of measurements, large prediction intervals were determined for $W Z$ surface before plasma processing. After plasma processing a significant decrease of prediction intervals for the $B A S E, W Z$, and $H A Z$ surfaces can be observed. This indicates that a high SEY uniformity surface layer was formed as consequence of plasma processing.

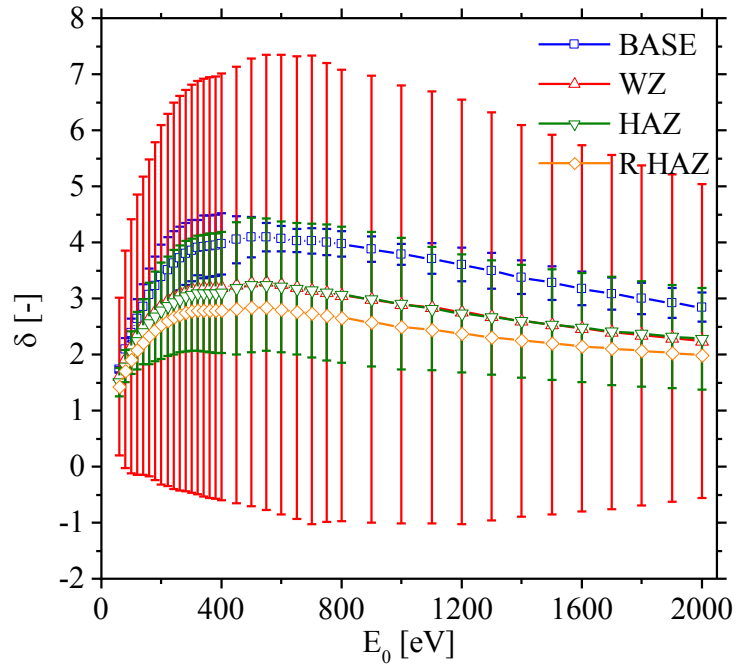

(a)

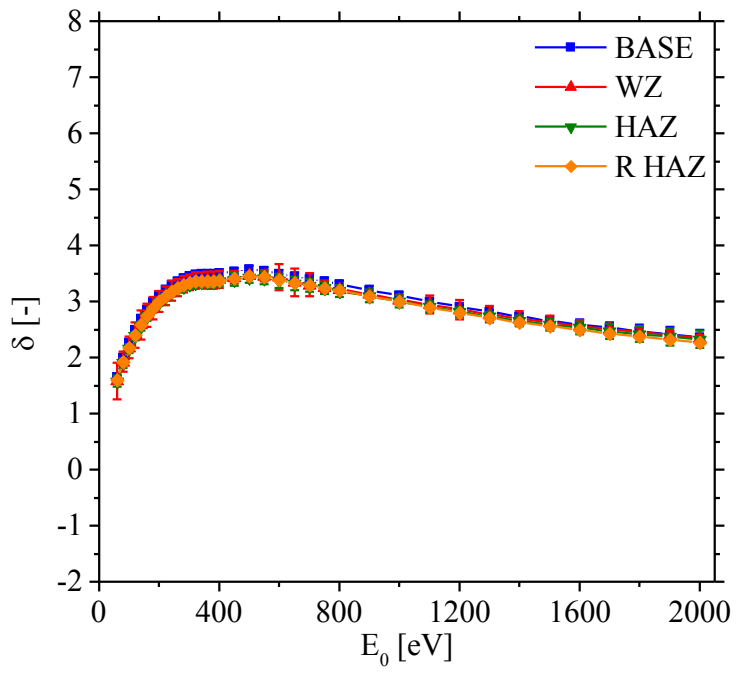

(b)

Fig. 5.24 The average $S E Y$ with $90 \%$ prediction interval bars as a function of $E_{0}$ at $\theta=-60^{\circ}$, (a) before and (b) after plasma processing.

\subsubsection{0 $S E Y$ as a function of incident angle of primary electrons}

The effect of the incident angle of primary electrons on characteristic surfaces was determined by plotting the $S E Y$ values measured at different incident angles for a single primary electron energy. 
A cosine law [27] used for modeling the influence of incident angle of electrons was fitted to the experimental results. The secondary electron yield measured at $E_{0}=320 \mathrm{eV}$ are plotted as a function of the incident angle of primary electron beam in the range from $-60^{\circ}$ to $+60^{\circ}$ (Fig. 5.25Fig. 5.28), before and after plasma processing. It was observed that the increase in the incident angle of primary electrons was followed by the increase in $S E Y$. This can be contributed to the formation of secondary electrons closer to the surface, which have a higher probability of leaving the surface of material.

Equation (4.5) is a function of the $S E Y$ at normal incidence, incident angle $\theta$, and the parameter $\eta$ which is a material dependent parameter. For each characteristic surface, the parameter $\eta$ was determined as the value that provides the best fit of the experimental results. The values of parameter $\eta$ given in Table 5.10 are obtained by minimizing the residual of the average $S E Y$ value of measurements and $S E Y$ value predicted by the Eq. (4.5). The range of $\eta$ across characteristic surfaces for samples prior to plasma processing is much larger than for samples after plasma processing. This indicates that after plasma processing, samples have more uniform surface layer across the characteristic surfaces. Highest value of $\eta$ was determined for $B A S E$ surface, while the differences between the weld area surfaces were much smaller.

Table 5.10 Values of parameter $\eta$ for characteristic surfaces, (a) before and (b) after plasma processing.

\begin{tabular}{|c|c|c|}
\hline \multirow{2}{*}{$\begin{array}{c}\text { Characteristic } \\
\text { surface }\end{array}$} & \multicolumn{2}{|c|}{$\eta$} \\
\hline & (a) & (b) \\
\hline$B A S E$ & 0.731 & 0.388 \\
\hline$W Z$ & 0.331 & 0.318 \\
\hline$H A Z$ & 0.256 & 0.267 \\
\hline$R-H A Z$ & 0.279 & 0.399 \\
\hline
\end{tabular}




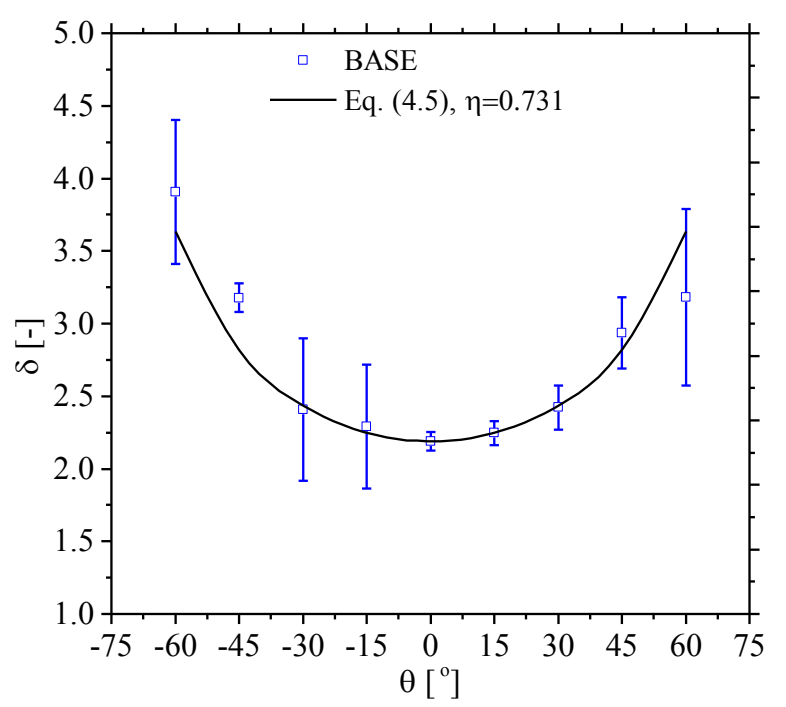

(a)

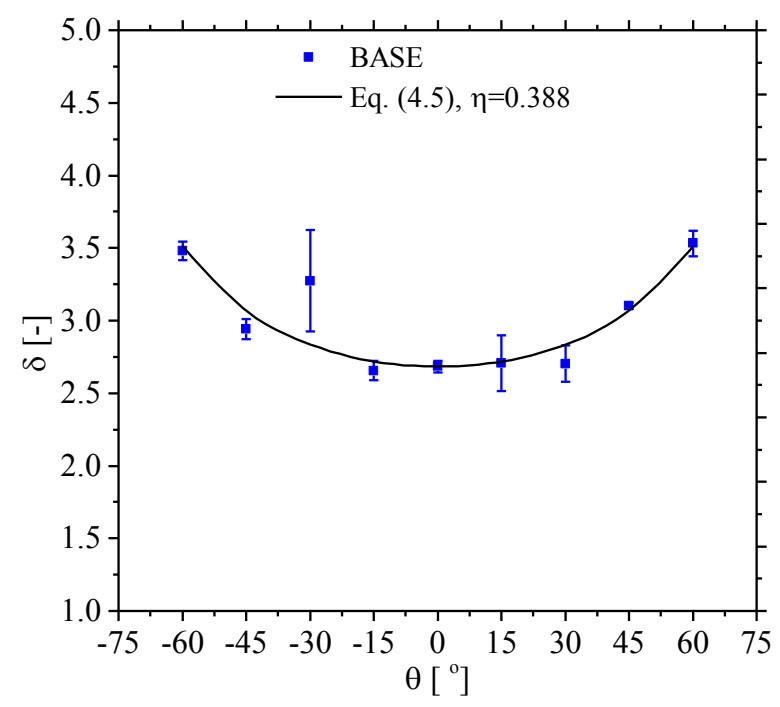

(b)

Fig. 5.25 $S E Y$ as a function of $\theta$ for $E_{0}=320 \mathrm{eV}$ of $B A S E$ surface, (a) before and (b) after plasma processing. Statistical bars represent the range of $90 \%$ prediction interval.

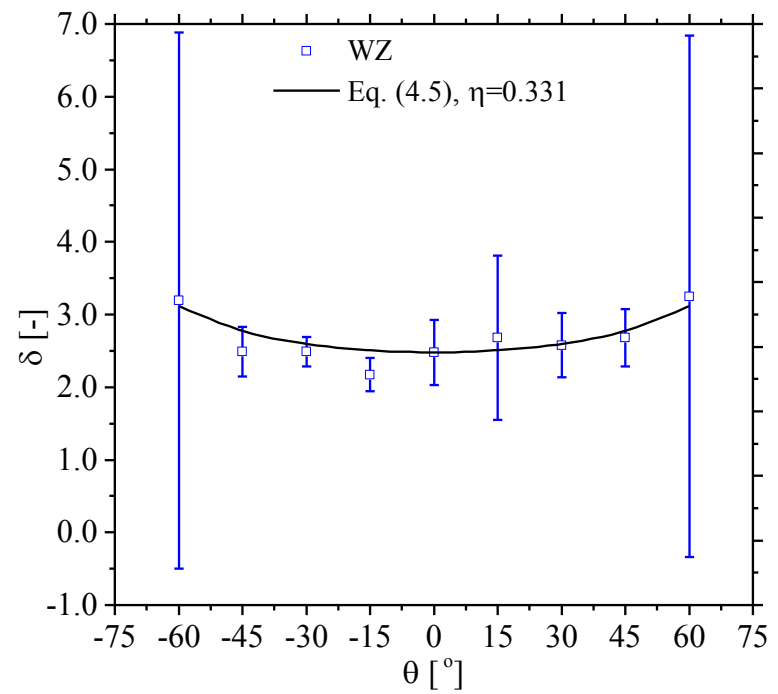

(a)

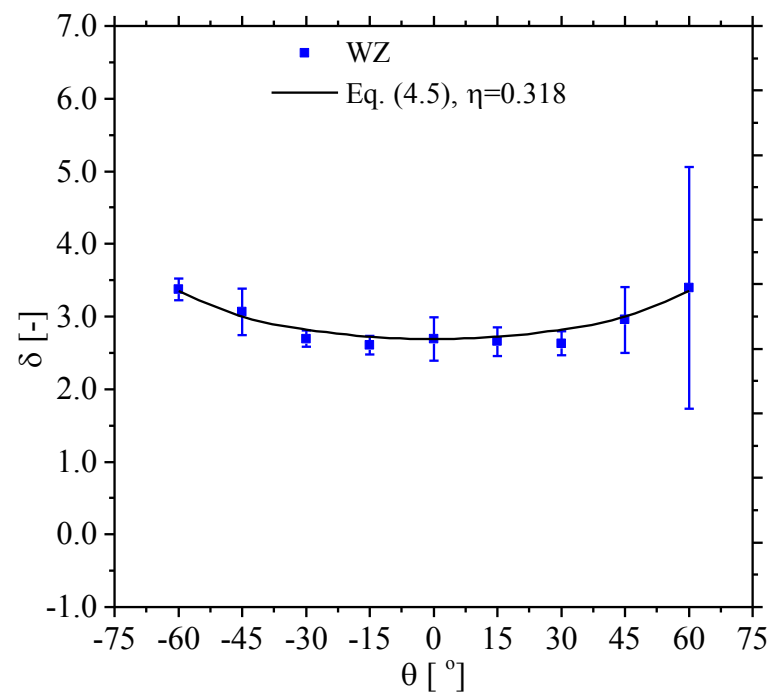

(b)

Fig. 5.26 $S E Y$ as a function of $\theta$ for $E_{0}=320 \mathrm{eV}$ of $W Z$ surface, (a) before and (b) after plasma processing. Statistical bars represent the range of $90 \%$ prediction interval. 




(a)

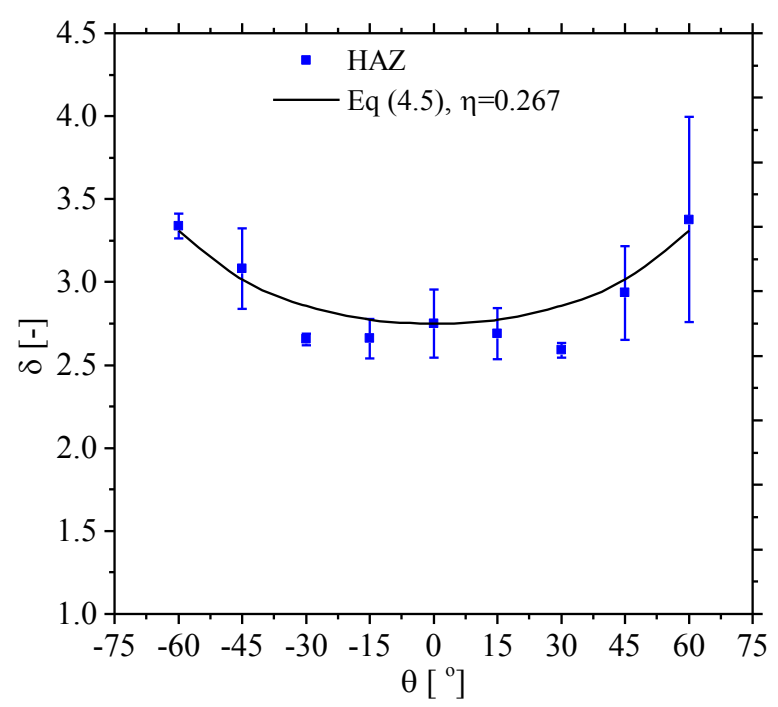

(b)

Fig. $5.27 S E Y$ as a function of $\theta$ for $E_{0}=320 \mathrm{eV}$ of $H A Z$ surface, (a) before and (b) after plasma processing. Statistical bars represent the range of $90 \%$ prediction interval.

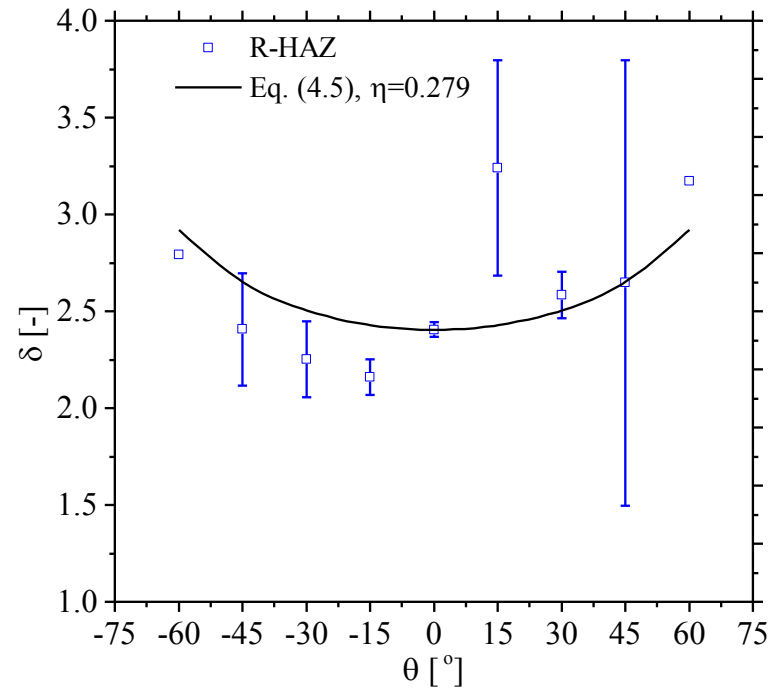

(a)

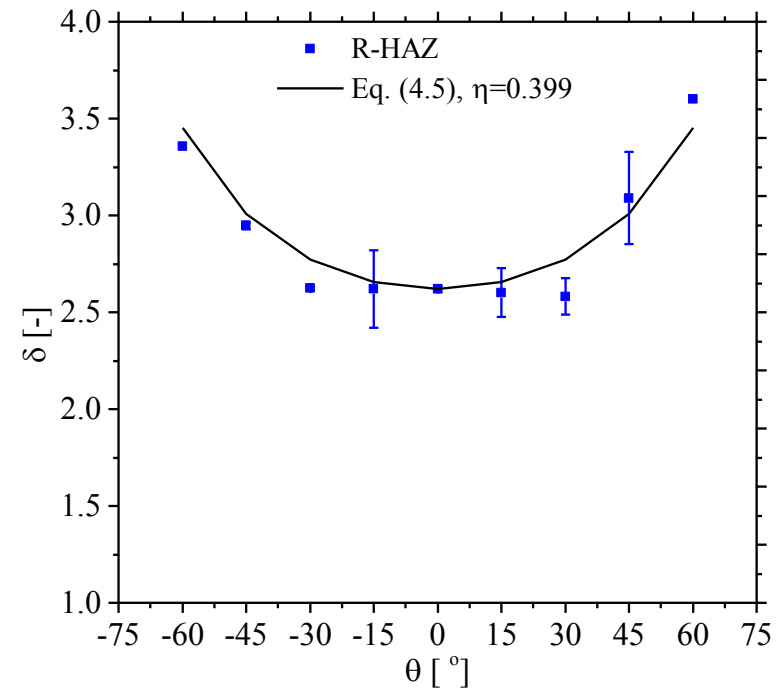

(b)

Fig. 5.28 SEY as a function of $\theta$ for $E_{0}=320 \mathrm{eV}$ of $R-H A Z$ surface, (a) before and (b) after plasma processing. Statistical bars represent the range of $90 \%$ prediction interval. 


\subsubsection{Properties of the characteristic surfaces}

The surface characterizations were performed on the $B A S E, W Z$, and $H A Z$ sample surfaces to determine the effect of $\mathrm{Ar} / \mathrm{O}_{2}$ plasma processing on the surface roughness, microstructure, and chemical composition. The surface roughness was measured with the atomic force microscope $(A F M)$. The scanning electron microscope (SEM) was used to determine the surface microstructure. The chemical composition of the surface layer was measured by energydispersive $x$-ray spectroscopy $(E D S)$.

Table 5.11 Surface statistics measured on a $50 x 50 \mu \mathrm{m}$ surface area by $A F M$ on characteristic surfaces, (a) before and (b) after plasma processing.

\begin{tabular}{cccc}
\hline \hline & & (a) & (b) \\
\hline \multirow{3}{*}{$B A S E$} & Mean roughness & $105.13 \mathrm{~nm}$ & $119.10 \mathrm{~nm}$ \\
& Max. height & $1.018 \mu \mathrm{m}$ & $1.109 \mu \mathrm{m}$ \\
& Surface area & $2539.8 \mu \mathrm{m}^{2}$ & $2512.3 \mu \mathrm{m}^{2}$ \\
\hline \multirow{3}{*}{$W Z$} & Mean roughness & $136.06 \mathrm{~nm}$ & $175.67 \mathrm{~nm}$ \\
& Max. height & $1.185 \mu \mathrm{m}$ & $1.342 \mu \mathrm{m}$ \\
& Surface area & $2540.0 \mu \mathrm{m}^{2}$ & $2520.1 \mu \mathrm{m}^{2}$ \\
\hline \multirow{3}{*}{$H A Z$} & Mean roughness & $95.941 \mathrm{~nm}$ & $178.70 \mathrm{~nm}$ \\
& Max. height & $1.131 \mu \mathrm{m}$ & $1.158 \mu \mathrm{m}$ \\
& Surface area & $2509.1 \mu \mathrm{m}^{2}$ & $2513.5 \mu \mathrm{m}^{2}$ \\
\hline
\end{tabular}

Surface roughness of the $B A S E, W Z$, and $H A Z$ was measured by $A F M$ on a surface area of $50 \times 50 \mu \mathrm{m}$ (Table 5.11). Plasma processing in $\mathrm{Ar} / \mathrm{O}_{2}$ had no effect on the global surface roughness of the characteristic surfaces measured by $A F M$. Because of the small surface of the measurement, it is not feasible to repeat the scan on the same exact spot on the sample before and after plasma processing. Also, due to the limitation in the vertical motion of the $A F M$ scanning probe, measurements were restricted to the areas where the roughness was within the capabilities of the microscope. The mean roughness measured on characteristic surfaces of 
samples before and after plasma processing indicate that there is no change in the surface roughness due to plasma processing.

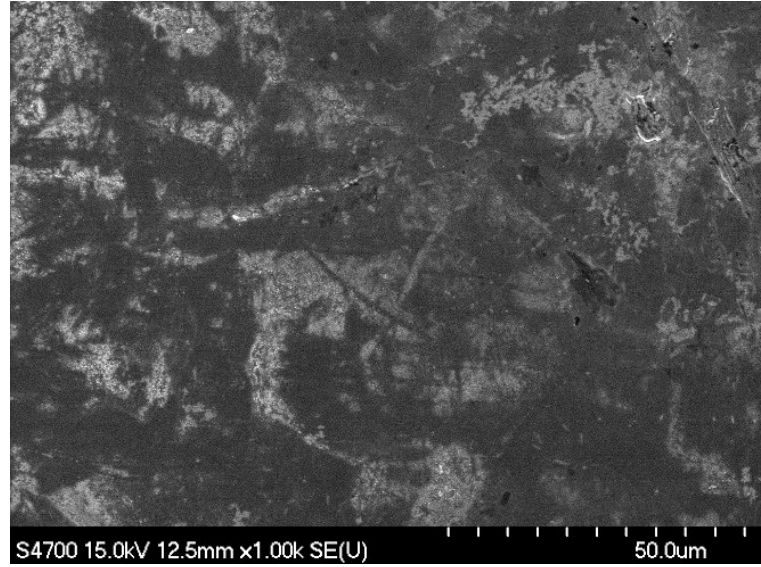

(a)

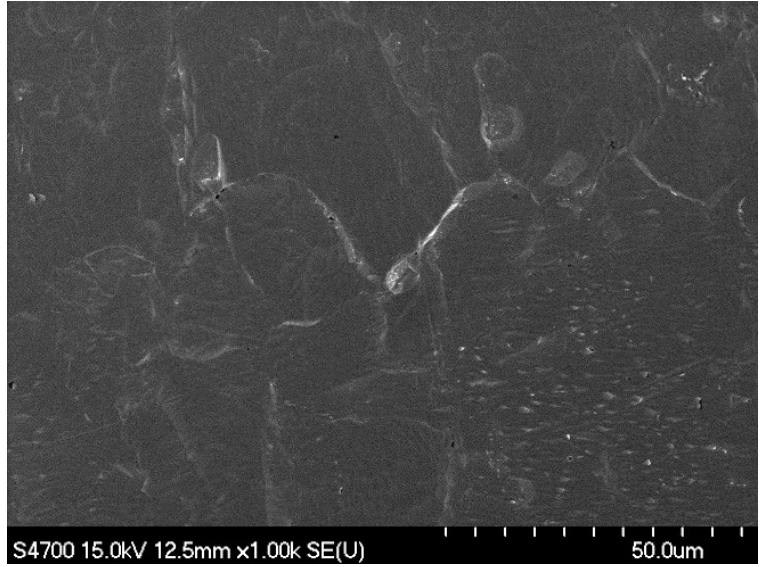

(b)

Fig. 5.29 SEM micrographs of BASE surface at x1,000 magnification, (a) before and (b) after plasma processing. Granular inclusions on the surface (a) were almost completely removed by processing in $\mathrm{Ar} / \mathrm{O}_{2}$ plasma for 1 hour.

SEM micrographs were taken at $B A S E, W Z$, and $H A Z$ surfaces before and after plasma processing. Before plasma processing, the SEM micrographs revealed the presence of submicrometer size inclusions on the BASE surface (Fig. 5.29 (a)). Furthermore, the BASE surface was more densely populated with the inclusions than the $H A Z$, while the $W Z$ had no trace of them. Only the micrographs of the $B A S E$ surface were included because of the large density of inclusions and the same effect in removal of the inclusions after plasma processing on all characteristic surfaces. Decreasing surface density of these inclusions towards the $W Z$ may be due to the effect of the elevated temperature during welding procedure. SEM measurements performed after plasma processing indicated that granular inclusions were almost completely 
removed from $B A S E$ surface, either by mechanical sputtering with argon ions or by chemical reaction with oxygen ions (Fig. 5.29 (b)).

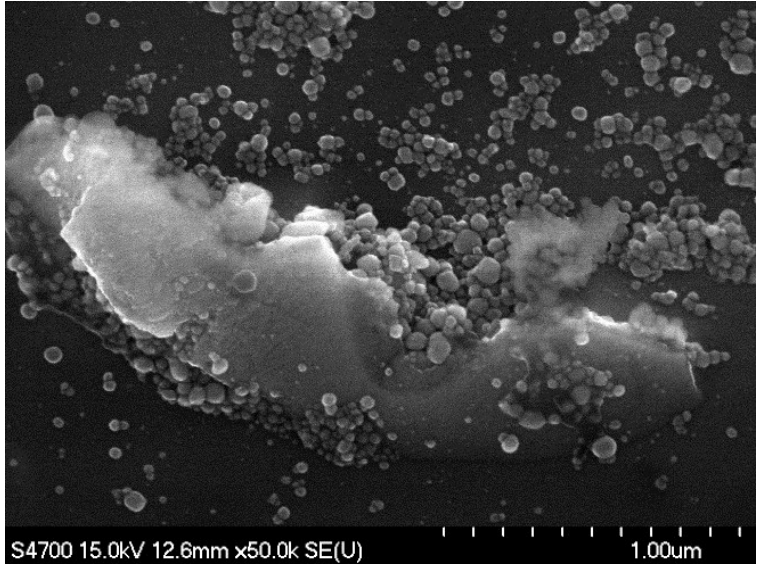

(a)

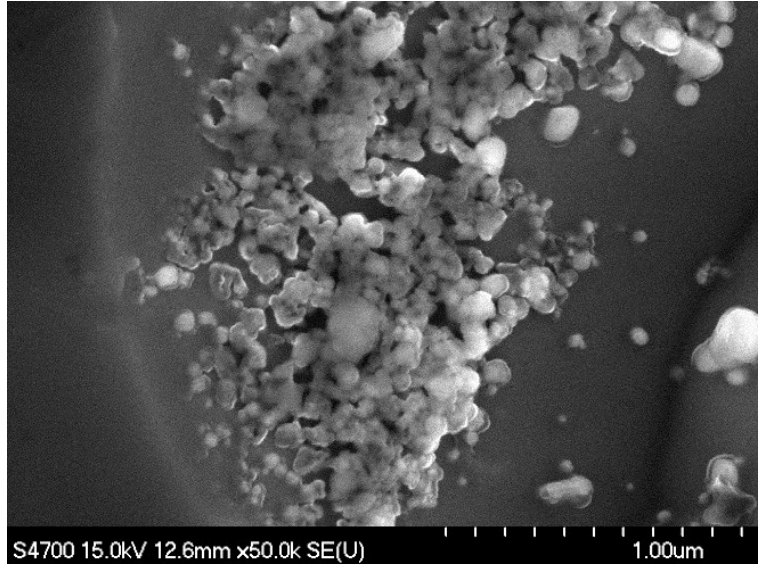

(b)

Fig. 5.30 SEM micrographs of the BASE surface at x50,000 magnification, (a) before and (b) after plasma processing.

A $S E M$ with a $\times 50,000$ magnification was used to characterize the granular inclusions. A micrograph of a group of granular inclusions on a $B A S E$ surface before plasma processing is shown in Fig. 5.30 (a). It was observed that the surface was covered with sub-micrometer inclusions which can form larger inclusions, completely covering the base surface. After plasma processing, a SEM picture was taken at the same magnification and of one of the few remaining inclusions (Fig. 5.30 (b)). Since the majority of the granular inclusions were removed (Fig. 5.29), this indicates that the plasma was effective in removing the contaminants from the surface layer.

$E D S$ measurements were also performed to determine the elemental composition of the observed area. EDS was performed directing the $x$-rays at the larger cluster of granular inclusions. The spectrum revealed high presence of niobium, and smaller content of silicon and 
oxygen (Fig. 5.31). This leads to a conclusion that the observed granular inclusions are silicon oxide. The number of $E D S$ counts is not the same before and after plasma processing (Fig. 5.31), however the ratio of the two highest niobium peaks are approximately the same. To compare the oxygen content of the granular inclusion, the ratio of oxygen peak and the maximum niobium peak were calculated, before and after plasma processing. After plasma processing, the relative elevation of the oxygen content can be observed in the remaining granular inclusions (Fig. 5.31 (b)).

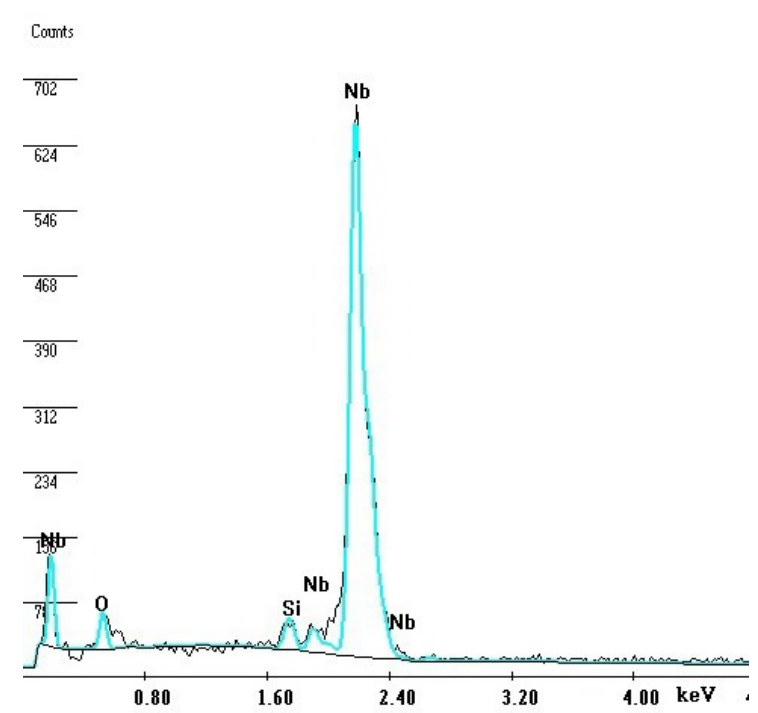

(a)

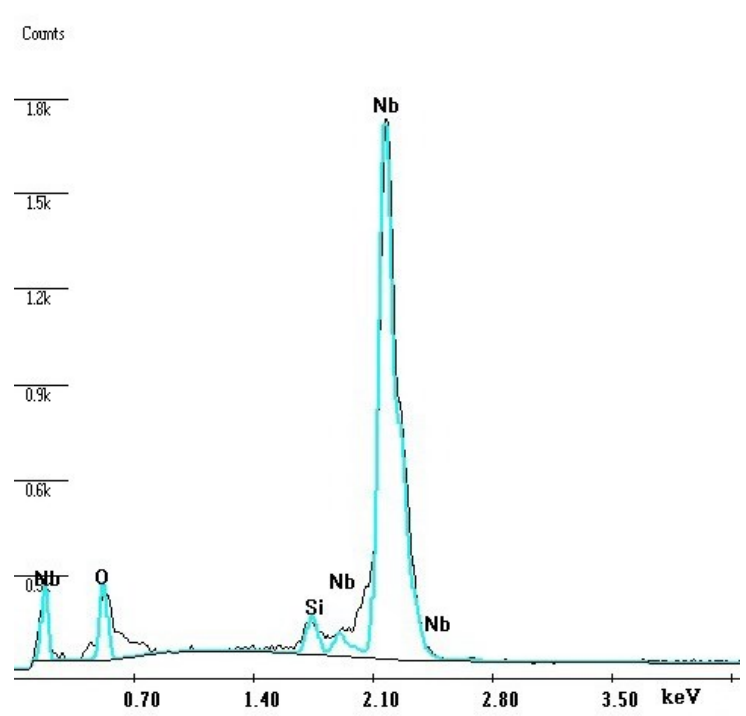

(b)

Fig. 5.31 EDS spectra measured at the high concentration of granular inclusions on $B A S E$ surface, (a) before and (b) after plasma processing. A relative increase in the oxygen content can be observed after plasma processing.

Larger areas of sample characteristic surfaces are measured by EDS to obtain chemical composition of sample surface before and after plasma processing. Before plasma processing, the $E D S$ of the $B A S E$ surface did not show a significant content of oxygen (Fig. 5.32 (a)). After 
plasma processing, oxygen was present on the surface of the sample (Fig. 5.32 (b)). An increase in oxygen content could have occurred during one or both of the two steps in the experimental process. The first opportunity was during the sample cooldown in plasma processing chamber which was done in an $\mathrm{Ar} / \mathrm{O}_{2}$ atmosphere. The second opportunity was during the exposure of the sample to the air at atmospheric pressure prior to the EDS measurement. An observed increase in the $S E Y$ after plasma processing can be related to the higher oxygen content on the surface.

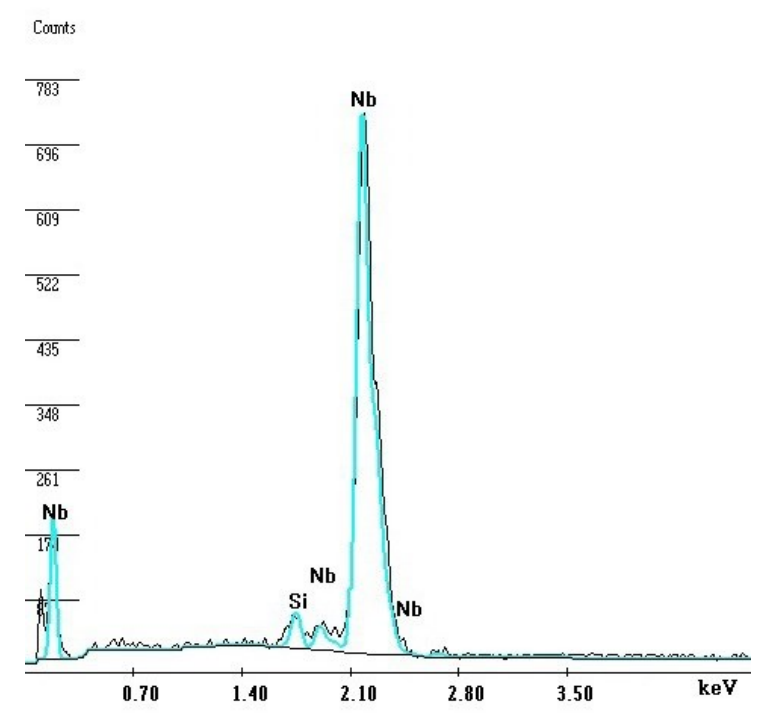

(a)

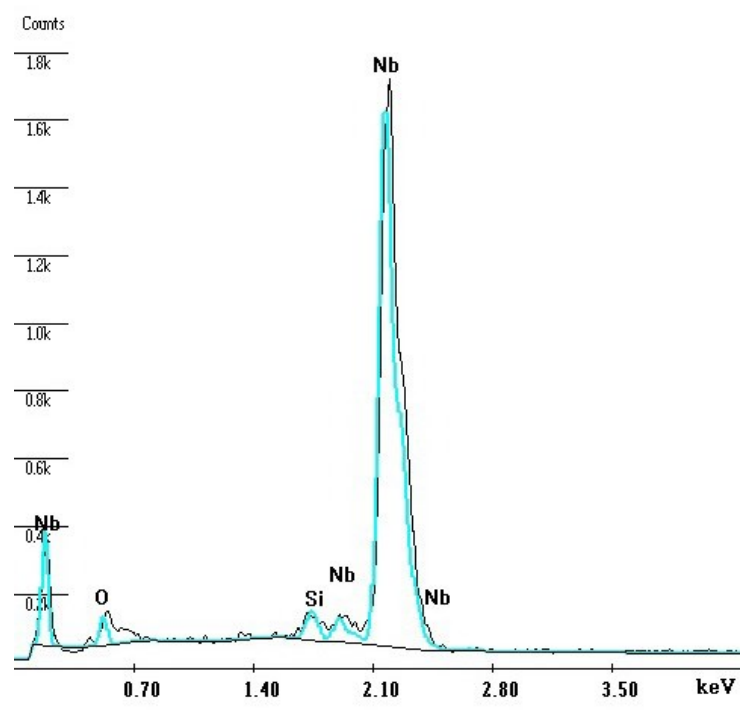

(b)

Fig. 5.32 EDS spectra measured on a characteristic BASE surface, (a) before and (b) after plasma processing. A spectral line after plasma processing indicates an increase in oxygen content on the BASE surface.

\subsection{Energy distribution curves}

The energy distribution curve $(E D C)$ of the emitted electrons was determined for several different primary electron energy levels at a normal incident angle of the primary electrons. 
The retarding electric field can be created around the collector by placing a high voltage power supply in the collector electric circuit (see Fig. 4.14). When the retarding potential exceeds the energy of the primary electron beam, all electrons emitted from the sample are completely reflected by the collector. With the incremental reduction of the retarding potential, an ever larger number of emitted electrons can impact the surface of the collector. $E D C$ measurements were performed at three primary electron beam energies $(140,180$, and $300 \mathrm{eV})$, but the obtained results were unreliable due to the formation of the tertiary electrons on the surface of the collector.

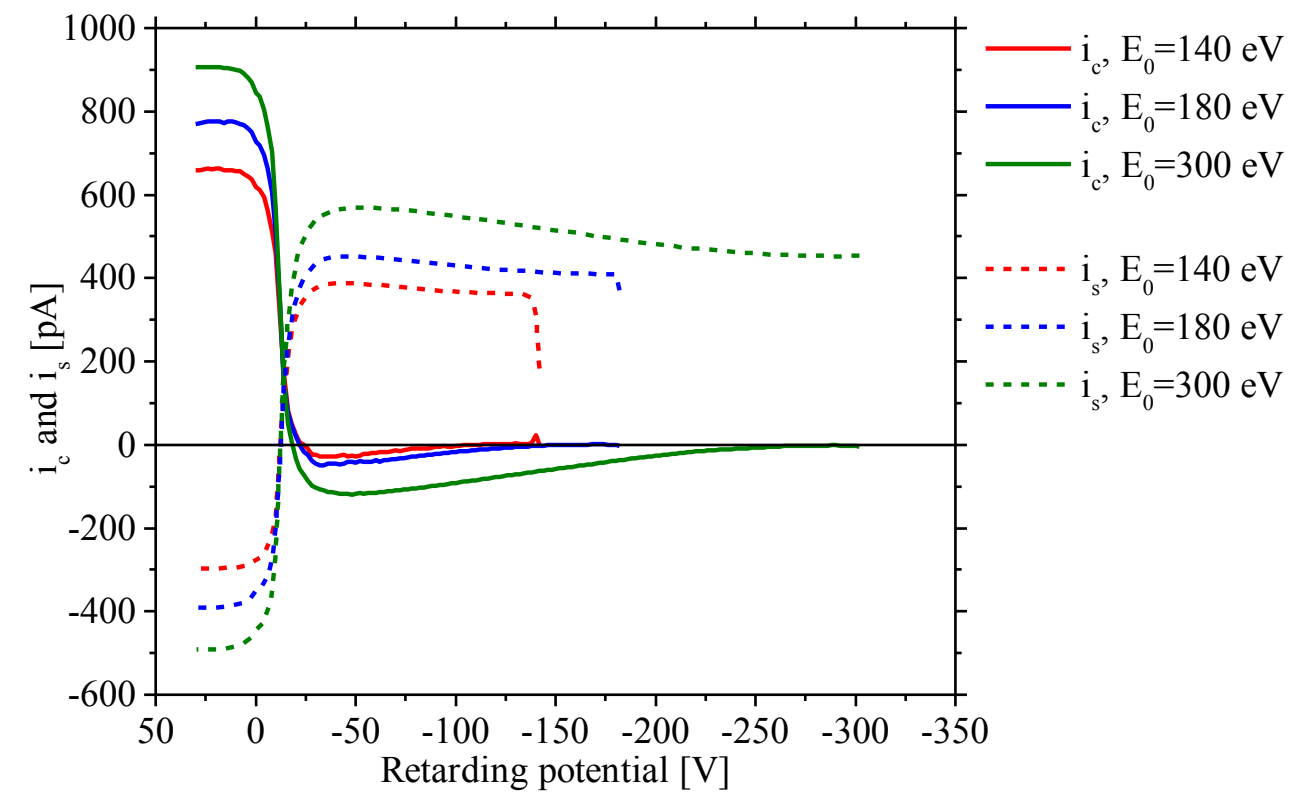

Fig. 5.33 Current measured on the collector $\left(i_{c}\right)$ and the BASE sample $\left(i_{s}\right)$ for indicated $E_{0}$, as a function of the collector retarding potential.

Energy distribution curves were determined for three primary electron beam energies at 140,180 , and $300 \mathrm{eV}$. The initial retarding potential values on the collector were 142, 182, and 
$302 \mathrm{~V}$, respectively. The highest retarding potential applied to the collector was always 2 volts higher than the primary electron beam energy in order to ensure that all electrons are reflected from the collector surface. The retarding potential was gradually reduced in increments of 2 volts, while the collector and sample current were measured after each incremental reduction of the voltage. EDC measurements always ended at $+30 \mathrm{~V}$ on the collector when the current measured on the collector and sample stabilized, while voltage of the sample was kept at $-10 \mathrm{~V}$ at all times. Once the retarding potential reached $0 \mathrm{~V}$, polarity of the power supply was reversed and the voltage was increased in $2 \mathrm{~V}$ increments to $+30 \mathrm{~V}$. Electron beam parameters used to measure the energy distribution curves are the same as for measurement of SEY.

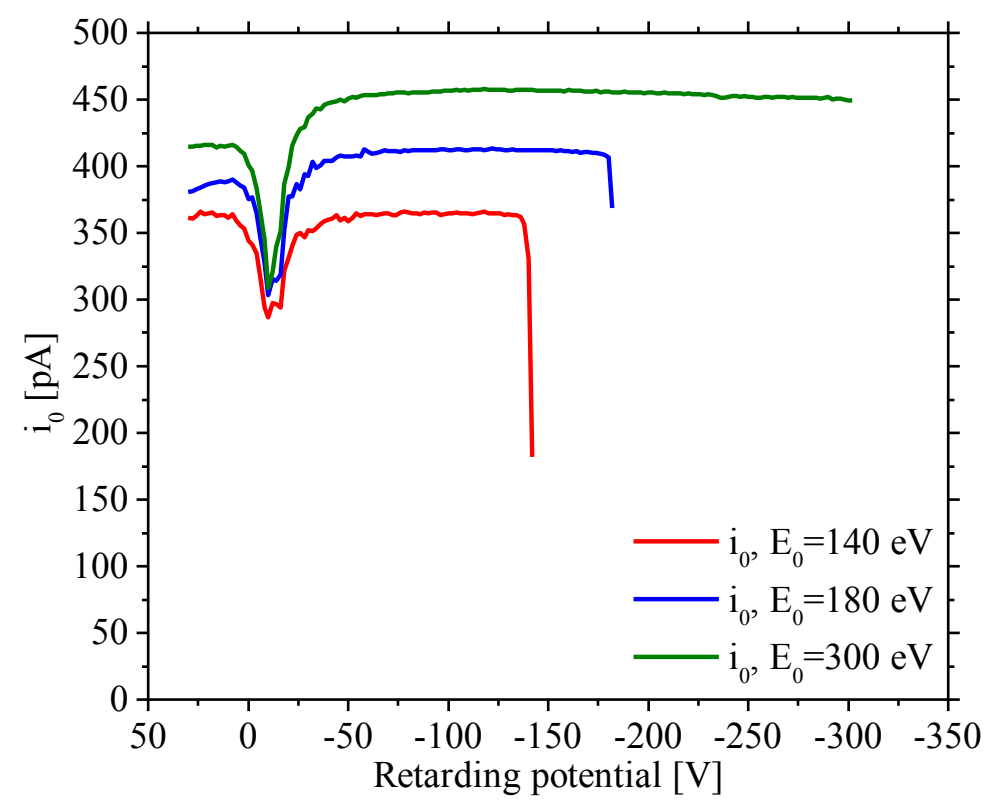

Fig. 5.34 Primary electron beam current $\left(i_{0}\right)$ calculated as the sum of collector and BASE sample currents for indicated $E_{0}$, as a function of the collector retarding potential. 
Directions and corresponding signs of the current were adopted as follows: a positive current indicates the inflow of electrons to the surface where the current was measured, while the negative current implies outflow of the electrons from the surface. For example, the measured currents on the collector and the BASE sample as a function of the collector retarding potential are shown in Fig. 5.33. The primary electron beam current was determined as the sum of collector and samples currents (Fig. 5.34). It can be observed that a sudden drop in the primary electron beam current occurs at the highest value of the collector retarding potential. This is due to the high retarding potential which caused part of the electrons coming from the electron gun to be blocked while passing through the aperture of the collector. All of the electrons started passing through the collector aperture after the retarding potential became equal to or lower than the primary electron beam energy. At that moment, the beam current became constant. In addition, the primary electron beam current suddenly dropped when both the collector and the sample were at the same potential $(-10 \mathrm{~V})$. The curve of the current magnitudes on the collector, the samples, and the primary electron beam as a function of retarding potential shown in Fig. 5.33 and Fig. 5.34 were also observed on other characteristic surfaces ( $W Z$ and $H A Z$ ). 


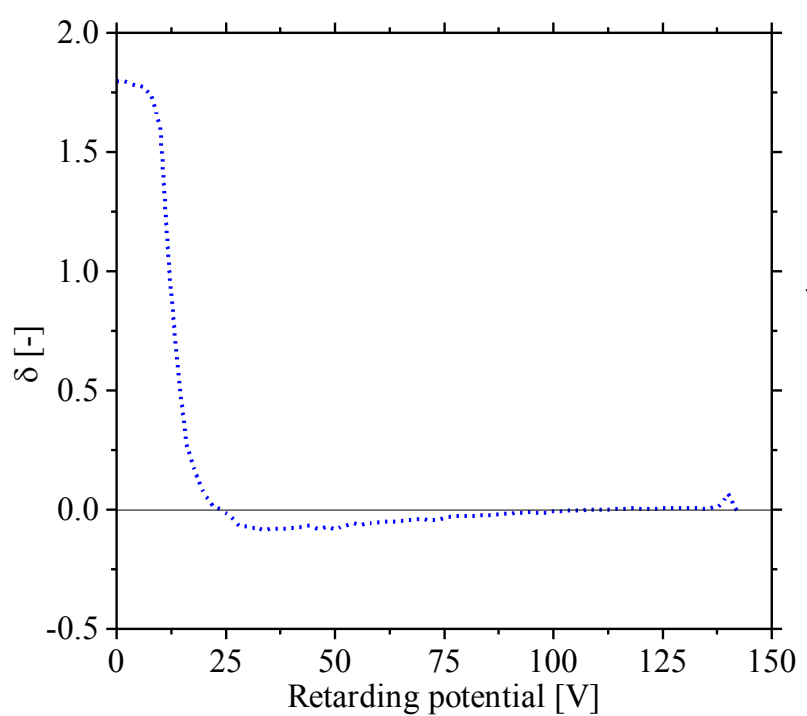

(a)

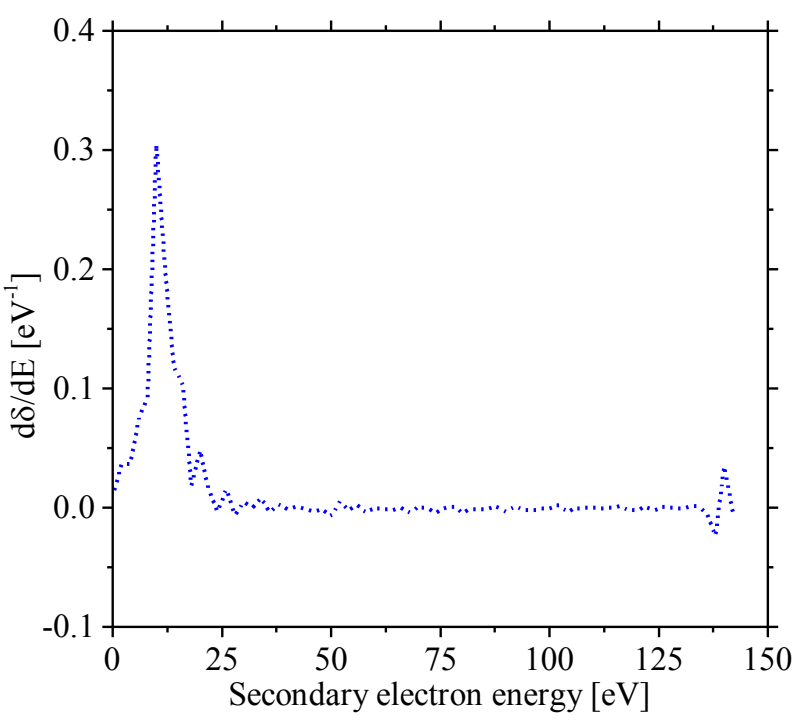

(b)

Fig. 5.35 (a) Normalized collector current as a function of retarding potential and (b) corresponding $E D C$, at $E_{0}=140 \mathrm{eV}$.

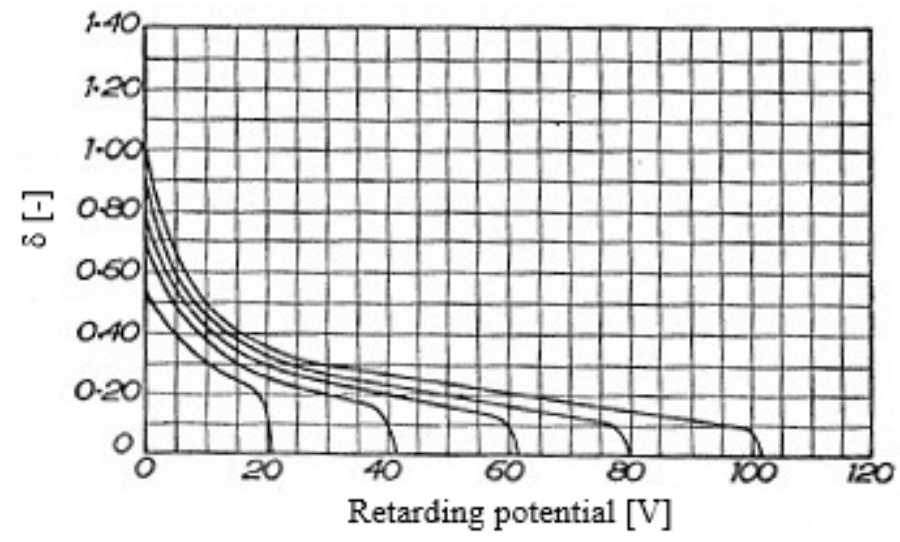

Fig. 5.36 Normalized collector current as a function of the retarding potential for copper sample $[56]$.

The first step in determining the $E D C$ was to normalize the current measured on the collector $\left(i_{c}\right)$ to primary electron beam current $\left(i_{0}\right)$. The second step was to apply a finite difference method on the normalized current function to determine the $E D C$. The results for 
normalization and the $E D C$ of emitted electrons from the $B A S E$ surface for $E_{0}=140 \mathrm{eV}$ are presented in Fig. 5.35.

To the best of knowledge of the author, there are no published results of normalized collector current curve for a niobium surface. The normalized collector current curves for copper were published in [56]. Normalized collector current curves determined in this research cannot be directly compared with the results in [56] due to the difference in the sample material and the fact that the measurements were made at different primary electron energy level since they were not measured on the same meter at the sample primary electron energy level. By comparing the trends of the curves reported in Fig. 5.35 (a) and Fig. 5.36, it can be observed that there are several differences. First, the results match when the retarding potential was larger than the energy of the primary electrons. Second, at the point where the retarding potential was the same as the primary electron energy, there was an increase in normalized current. As the retarding potential was further reduced to zero, collector current continued to rise slowly (Fig. 5.36). The current on the collector gradually increased as the true secondary electrons were allowed to reach the collector surface. On the other hand, after the initial peak at primary electron energy, the collector current drops back to zero (Fig. 5.35 (a)). As the retarding potential was further reduced, the reversal of the collector current direction was observed until the retarding potential was reduced to $20 \mathrm{~V}$, when compared to the current direction in Fig. 5.36. The reversed current direction indicated that the number of electrons leaving the surface of the collector was larger than the number of arriving electrons. Electrons leaving the surface of the collector are called tertiary electrons. It can be noticed that current on the sample increased with the same magnitude but in the opposite direction as the current on the collector. That leads to a conclusion that the electrons leaving the surface of the collector are landing on the sample. Below the critical 
retarding potential, the number of true secondary electrons reaching the surface of the collector counteracts the tertiary electrons leaving the collector surface.

The $E D C$ is accurately measured by retarding field analyzer $(R F A)$, which has several grids under different potentials to reflect formed tertiary electrons. The EDCs measured with the existing experimental setup can only be used qualitatively to provide an insight on the effect of plasma on the energy distribution of the electrons reflected and emitted by the sample.

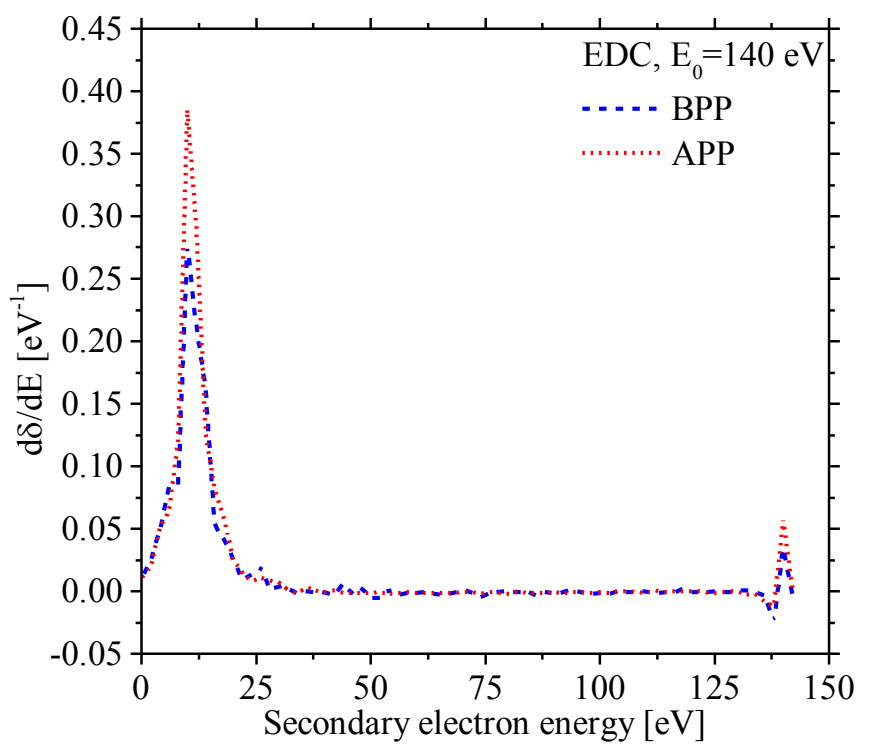

Fig. 5.37 EDC of $B A S E$ sample measured before $(B P P)$ and after $(A P P)$ plasma processing as a function of secondary electron energy at $E_{0}=140 \mathrm{eV}$.

The $E D C$ for $140 \mathrm{eV}$ of primary electron energy was obtained by measuring the cumulative current on the collector. The measurements were performed on the $B A S E$ sample at normal incident angle of the primary electrons. The collector current was normalized with respect to the total current and differentiated to determine energy distribution of the emitted electrons. It can be observed that $E D C$ at this energy level of primary electrons has two distinct peaks (Fig. 5.37). 
The first peak appears at $140 \mathrm{eV}$ energy. This peak indicates the elastically reflected electrons since their energy is equal to the energy of the primary electrons. With the further decrease of the retarding potential, the second peak appears at the $10 \mathrm{eV}$ of the electron energy, i.e. when the true secondary electrons had enough energy to overcome the retarding potential of the collector.



Fig. $5.38 E D C$ of $B A S E$ sample measured before $(B P P)$ and after $(A P P)$ plasma processing as a function of secondary electron energy at $E_{0}=180 \mathrm{eV}$.

The EDC measurement was repeated on the same sample after plasma processing. The observed peaks appeared in the same energy values as before the plasma processing but with higher intensities (Fig. 5.37). Similar observations are made in $E D C$ for primary electron energies of 180 and $300 \mathrm{eV}$ but with one significant distinction, namely, the peak that indicates the elastically reflected electrons was not detected (Fig. 5.38 and Fig. 5.39). With the increase of the primary electron energy, the number of elastically reflected electrons is reduced [57]. On the other hand, the intensity of the true secondary electron peak becomes higher with the primary 
electron energy increase. At the same primary electrons energy, the true secondary electron peak had a higher magnitude after plasma processing. The increase of the peaks on the $E D C$ curves after plasma processing coincides with the observed increase in $S E Y$, at all three primary electrons energies. Similar observations were made on the $W Z$ and $H A Z$ surfaces.

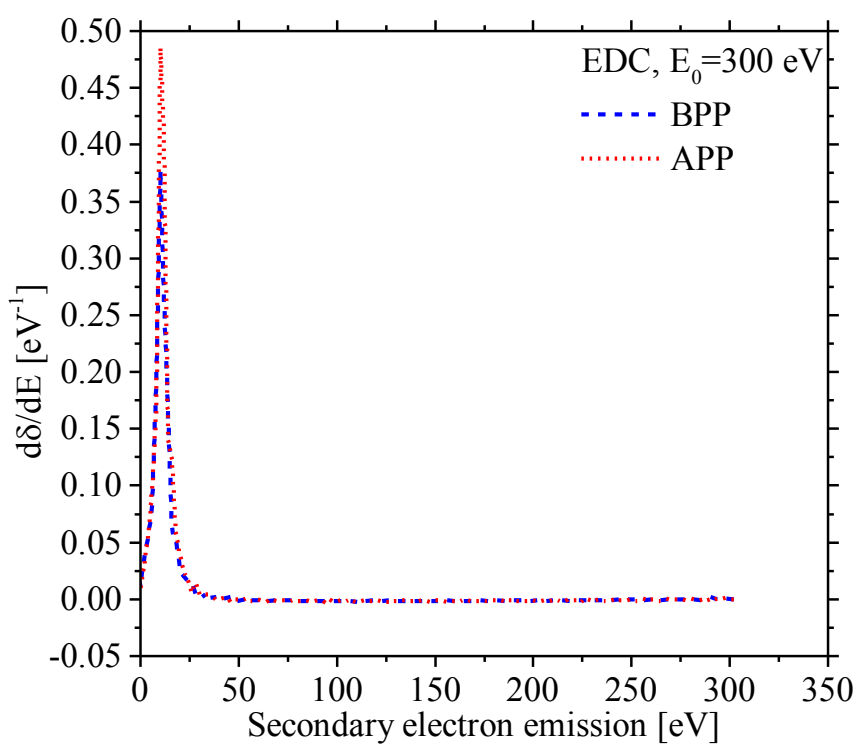

Fig. 5.39 $E D C$ of $B A S E$ sample measured before $(B P P)$ and after $(A P P)$ plasma processing as a function of secondary electron energy at $E_{0}=300 \mathrm{eV}$.

An attempt was made to measure the EDC of the electrons emitted from the surface of the sample by applying the high retarding potential on the collector and measuring the current while gradually reducing the retarding potential. Due to the effect of tertiary electrons formed on the surface of the collector, the results of these measurements are used only for qualitative analysis of the impact of the plasma processing. 


\section{CHAPTER 6}

\section{CONCLUSION}

An experimental setup was developed to observe the secondary electron emission at impacting electron energies in the range from 60 to $2000 \mathrm{eV}$, and different incident angles of primary electrons. The samples used for experiments were made from a niobium plate used for manufacturing $S R F$ cavities. A sample manipulating system developed for observing the effects of the electrons impacting the surface at different angles allowed the measurement of incident angles in the range from $-60^{\circ}$ to $+60^{\circ}$ with the $15^{\circ}$ angle increment. Three sets of samples, representative of three different types of microstructures found in $S R F$ cavities, were used in this work to study SEE of characteristic surfaces. One of the sample sets was made to represent the base surface of the niobium cavity, with a microstructure representative of cold work deformation which was not affected by the electron beam welding. The other two sets of samples had several characteristic surfaces identical to the weld area of the cavity, with microstructures affected by the heat during electron beam welding process. Characteristic surfaces included the weld zone (WZ), heat affected zone (HAZ), and base material surface which was divided into heat treated surface $(R-H A Z)$ adjacent to the heat affected zone and non-treated surface of niobium $(B A S E)$. The position of the characteristic surfaces on each sample set was determined and SEY measurements were performed. Secondary electron yield results obtained from the similar characteristic surfaces between different sample sets were combined.

The susceptibility of the $S E Y$ magnitude to repeated electron beam exposure was measured on the $B A S E$ and $W Z$ surface. Seven $S E Y$ measurements were performed on a single spot of both 
surfaces at normal incident angle of primary electrons. A decrease in SEY magnitude was observed after each measurement indicating that the electron beam was modifying the surface of the sample. After completing the $S E Y$ measurement, samples were placed in a commercial plasma cleaning system where they were processed for one hour by ionized nitrogen gas. Following plasma processing, additional seven $S E Y$ measurements were performed to test the effect of plasma processing on the $S E Y$ magnitude. A substantial reduction in the $S E Y$ change after each measurement was observed. On the other hand, the average $S E Y$ curve of the $B A S E$ surface after plasma processing was larger when compared to the average $S E Y$ before plasma processing. The increase of the $S E Y$ after plasma processing was contributed to the higher reactivity of the surface to the air at atmospheric pressure, which was also observed in previously published results.

The uniformity of the $S E Y$ across the surface was determined by taking measurements at different spots on the sample. The secondary electron yield variation of all characteristic surfaces was determined by averaging the results obtained from multiple measuring spots on the sample and calculating the sample standard deviation. Based on the preformed measurements, a prediction interval of possible $S E Y$ magnitudes on the characteristic surfaces was calculated at a 0.9 probability.

In addition, the influence of the incident angle of the primary electrons on the $S E Y$ of characteristic surfaces was determined. After completing the $S E Y$ measurements, samples were processed in an argon/oxygen gas mixture plasma for one hour. Following plasma processing, the $S E Y$ measurements were repeated on all samples and the results were compared with the results before plasma processing. The majority of the experiments showed a significant decrease in the $S E Y$ variation across the characteristic surfaces after plasma processing. It was also 
observed that after plasma processing the average $S E Y$ magnitudes of all characteristic surfaces coincide to a great degree.

Three different surface characterizations were performed on $W Z, H A Z$ and $B A S E$ surfaces, namely surface roughness, surface microstructure, and chemical composition.

The surface roughness was measured by an atomic force microscope on characteristic surfaces before and after plasma processing. No changes were observed in the mean roughness after plasma processing.

The surface microstructure was examined with the scanning electron microscope. An examination detected sub-micrometer granular inclusions on the surfaces of the samples before plasma processing. After plasma processing, most of the granular inclusions were removed.

The chemical composition of the characteristic surfaces was determined by an energydispersive $x$-ray spectroscopy $(E D S)$. The $E D S$ showed an increase in oxygen content of all characteristic surfaces, after plasma processing. This indicates the increase in the niobium surface reactivity with the air and formation of oxides after plasma processing.

The energy distribution of the reflected and emitted electrons was expressed with the energy distribution curve. An energy distribution curve was determined by measuring the current on the collector in a range of retarding potentials starting from the energy level of primary electrons up to $0 \mathrm{~V}$. Specifically, the retarding potential was reduced in $2 \mathrm{~V}$ increments while the current was measured at each value. To obtain $E D C$, a cumulative current was normalized by the primary electron beam current and differentiated with respect to the energy. The resulting curves were used to qualitatively describe the effect of plasma, due to the high influence of tertiary electrons formed on the surface of the collector. For all three primary electron energies $E D C$ peak of true secondary electrons was of higher intensity after plasma processing, indicating the increase in the 
quantity of low energy electrons emitted from the surface. The increase of true secondary electron quantity can be related to the observed increase of $S E Y$ after plasma processing.

The experimental results provided the insight on the effect of plasma processing on the surface of cavity grade niobium. The results showed beneficial effects of plasma on the decrease of susceptibility and the increase in uniformity of the $S E Y$ between characteristic surfaces of the sample. Specifically, the processing in nitrogen plasma resulted in a reduced variation of the $S E Y$ during repeated exposure of a single measurement spot to the electron beam. Processing in nitrogen plasma decreased the sensitivity of surface SEY to repeated electron beam exposure. Furthermore, the plasma processing of the samples in the $\mathrm{Ar} / \mathrm{O}_{2}$ gas mixture leveled the $S E Y$ magnitude across the characteristic surfaces.

Granular inclusions detected by $S E M$ on the surface before plasma processing were identified as a silicon oxide, based on the EDS measurement. The majority of the granular inclusions were removed by processing the surface in an $\mathrm{Ar} / \mathrm{O}_{2}$ plasma. Even though the oxides inclusions were removed, larger scale $E D S$ measurement showed an increased oxygen content. A higher oxygen content could be related to the observed increase of average $S E Y$ measured on all characteristic surfaces.

The experimental setup accomplished the set goals. However, the effect of air exposure on the samples during the transfer between the processing and measurement chamber and resulting SEY magnitudes were underestimated. As part of future work, air exposure of samples can be avoided by adding an additional chamber to the developed experimental setup, in which the plasma processing can be performed.

Namely an antechamber could be added to the $S E Y$ measurement chamber separated by a gate valve. This antechamber should be equipped with a gas flow system and a power supply 
which would allow the plasma processing of the samples in the experimental setup. At the same time, the sample manipulation system would have to be redesigned to allow the transfer of samples between the antechamber and the measurement chamber. The modified experimental setup would avoid the exposure of samples to air.

The reduction of the $S E Y$ magnitudes might be achieved by varying the plasma processing parameters with intent to remove inclusions detected on the surface of the samples. The experiments indicate that inclusions were not completely removed from the surface by plasma processing with applied process parameters. Extended plasma processing, or processing at higher power levels could potentially improve the effectiveness of plasma processing. The results presented in this work were obtained for a single combination of $r f$ power, duration of plasma processing, and pressure in the processing chamber for both $\mathrm{N}_{2}$ and $\mathrm{Ar} / \mathrm{O}_{2}$. The optimal set of processing parameters could be determined by performing a series of experiments with a range in magnitude of $r f$ power, duration of plasma processing, level of vacuum, and employed processing gases. 


\section{BIBLIOGRAPHY}

[1] Cockcroft, J. D., and Walton, E. T. S. "Experiments with high velocity positive ions. (I) Further developments in the method of obtaining high velocity positive ions," Proceedings of the Royal Society of London A: Mathematical, Physical and Engineering Sciences Vol. 136, No. 830, 1932, pp. 619-630.

[2] Upadhyay, J., Im, D., Peshl, J., Bašović, M., Popović, S., Valente-Feliciano, A. M., Phillips, L., and Vušković, L. "Apparatus and method for plasma processing of SRF cavities," Nuclear Instruments and Methods in Physics Research Section A: Accelerators, Spectrometers, Detectors and Associated Equipment Vol. 818, 2016, pp. 76-81. doi: http://dx.doi.org/10.1016/j.nima.2016.02.049

[3] Calder, R., Dominichini, G., and Hilleret, N. "Influence of various vacuum surface treatments on the secondary electron yield of niobium," Nuclear Instruments and Methods in Physics Research Section B: Beam Interactions with Materials and Atoms Vol. 13, No. 1-3, 1986, pp. 631-636. doi: http://dx.doi.org/10.1016/0168-583X(86)90581-1

[4] B. Aune, e. a. "Superconducting TESLA cavities," Physical Review Special Topics Accelerators and Beams Vol. 3, No. 9, 2000, p. 092001.

[5] Bardeen, J., Cooper, L., and Schrieffer, J. "Theory of superconductivity," Physical Review Vol. 108, No. 5, 1957, pp. 1175-1204.

[6] Farnsworth, P. T. "Television by electron image scanning," Journal of the Franklin Institute Vol. 218, No. 4, 1934, pp. 411-444. doi: http://dx.doi.org/10.1016/S0016-0032(34)90415-4

[7] Geng, R. L. "Multipacting simulations for superconducting cavities and RF coupler waveguides," Particle Accelerator Conference, 2003. PAC 2003. Proceedings of the. Vol. 1, 2003, pp. 264-268 Vol.1.

[8] Weingarten, W. "Electron loading," Proceedings of the Second Workshop on RF Superconductivity. Geneva, Switzerland, 1984, pp. 551-582.

[9] Knoblock, J. "Field emission and thermal breakdown in superconducting niobium cavities for accelerators," Applied Superconductivity, IEEE Transactions on Vol. 9, No. 2, 1999, pp. 1016-1022.

doi: $10.1109 / 77.783471$

[10] Hasan, P. "The science and technology of superconducting cavities for accelerators," Superconductor Science and Technology Vol. 14, No. 4, 2001, p. R28.

[11] Safa, H. "High field behavior of SCRF cavities," Proceedings of the Tenth Workshop on RF Superconductivity. Tsukuba, Japan, 2001, pp. 279-286.

[12] Saito, K. "Critical field limitation of the niobium superconductiong RF cavity," Proceedings of the Tenth Workshop on RF Superconductivity. Tsukuba, Japan, 2001, pp. 583-587.

[13] Austin, L., and Starke, H. "Ueber die reflexion der kathodenstrahlen und eine damit verbundene neue erscheinung secundärer emission," Annalen der Physik Vol. 314, No. 10, 1902, pp. 271-292.

doi: $10.1002 /$ andp. 19023141003 
[14] Furman, M., and Pivi, M. "Probabilistic model for the simulation of secondary electron emission," Physical Review Special Topics - Accelerators and Beams Vol. 5, No. 12, 2002, p. 124404.

[15] McKay, K. G. "Secondary electron emission," Advances in Electronics and Electron Physics. Vol. Volume 1, Academic Press, 1948, pp. 65-130.

[16] Shih, A., and Hor, C. "Secondary emission properties as a function of the electron incidence angle," Electron Devices, IEEE Transactions on Vol. 40, No. 4, 1993, pp. 824829.

doi: $10.1109 / 16.202797$

[17] Hilleret, N., Scheuerlein, C., and Taborelli, M. "The secondary-electron yield of airexposed metal surfaces," Applied Physics A Vol. 76, No. 7, 2003, pp. 1085-1091. doi: 10.1007/s00339-002-1667-2

[18] Bruining, H. "The depth at which secondary electrons are liberated," Physica Vol. 3, No. 9, 1936, pp. 1046-1052. doi: http://dx.doi.org/10.1016/S0031-8914(36)80330-9

[19] Le Pimpec, F., Kirby, R. E., King, F., and Pivi, M. "Properties of TiN and TiZrV thin film as a remedy against electron cloud," Nuclear Instruments and Methods in Physics Research Section A: Accelerators, Spectrometers, Detectors and Associated Equipment Vol. 551, No. 2-3, 2005, pp. 187-199. doi: http://dx.doi.org/10.1016/j.nima.2005.05.048

[20] Scheuerlein, C., Taborelli, M., Hilleret, N., Brown, A., and Baker, M. A. "An AES study of the room temperature conditioning of technological metal surfaces by electron irradiation," Applied Surface Science Vol. 202, No. 1-2, 2002, pp. 57-67. doi: http://dx.doi.org/10.1016/S0169-4332(02)00868-1

[21] Le Pimpec, F., Kirby, R. E., King, F. K., and Pivi, M. "The effect of gas ion bombardment on the secondary electron yield of TiN, TiCN and TiZrV coatings for suppressing collective electron effects in storage rings," Nuclear Instruments and Methods in Physics Research Section A: Accelerators, Spectrometers, Detectors and Associated Equipment Vol. 564, No. 1, 2006, pp. 44-50.

doi: http://dx.doi.org/10.1016/j.nima.2006.03.041

[22] Hartung, W. H., Asner, D. M., Conway, J. V., Dennett, C. A., Greenwald, S., Kim, J. S., Li, Y., Moore, T. P., Omanovic, V., Palmer, M. A., and Strohman, C. R. "In-situ measurements of the secondary electron yield in an accelerator environment: Instrumentation and methods," Nuclear Instruments and Methods in Physics Research Section A: Accelerators, Spectrometers, Detectors and Associated Equipment Vol. 783, 2015, pp. 95-109.

doi: http://dx.doi.org/10.1016/j.nima.2015.01.092

[23] Basovic, M., Samolov, A., Cuckov, F., Popović, S., M, T., and Vušković, L. "Secondary electron yield of electron beam welded areas of SRF cavities," Proceedings of the Seventeenth Workshop on RF Superconductivity Whistler, Canada, 2015.

[24] Bojko, I., Hilleret, N., and Scheuerlein, C. "Influence of air exposures and thermal treatments on the secondary electron yield of copper," Journal of Vacuum Science \& Technology A Vol. 18, No. 3, 2000, pp. 972-979.

doi: doi:http://dx.doi.org/10.1116/1.582286

[25] Kirby, R. "Artifacts in secondary electron emission yield measurements," SLAC-PUB10541, Stanford University, Stanford, CA 2004. 
[26] Hartung, W. H., Asner, D. M., Conway, J. V., Dennett, C. A., Greenwald, S., Kim, J.-S., Li, Y., Moore, T. P., Omanovic, V., Palmer, M. A., and Strohman, C. R. "Report on instrumentation and methods for in-situ measurements of the secondary electron yield in an accelerator environment," http://arxiv.org/abs/1407.0772, Cornell University Library, Ithaca, NY, 2014.

[27] Seiler, H. "Secondary electron emission in the scanning electron microscope," Journal of Applied Physics Vol. 54, No. 11, 1983, pp. R1-R18. doi: doi:http://dx.doi.org/10.1063/1.332840

[28] Han, J. H., Krasilnikov, M., and Flöttmann, K. "Secondary electron emission in a photocathode rf gun," Physical Review Special Topics - Accelerators and Beams Vol. 8, No. 3, 2005, p. 033501.

[29] Lin, Y., and Joy, D. C. "A new examination of secondary electron yield data," Surface and Interface Analysis Vol. 37, No. 11, 2005, pp. 895-900. doi: 10.1002/sia.2107

[30] Vaughan, J. R. M. "A new formula for secondary emission yield," IEEE Transactions on Electron Devices Vol. 36, No. 9, 1989, pp. 1963-1967. doi: $10.1109 / 16.34278$

[31] Bruining, H. "Secondary electron emission: Part II. Absorption of secondary electrons," Physica Vol. 5, No. 10, 1938, pp. 901-912. doi: http://dx.doi.org/10.1016/S0031-8914(38)80032-X

[32] Kirby, R. E., and King, F. K. "Secondary electron emission yields from PEP-II accelerator materials," Nuclear Instruments and Methods in Physics Research Section A: Accelerators, Spectrometers, Detectors and Associated Equipment Vol. 469, No. 1, 2001, pp. 1-12. doi: http://dx.doi.org/10.1016/S0168-9002(01)00704-5

[33] Yin Vallgren, C., Arduini, G., Bauche, J., Calatroni, S., Chiggiato, P., Cornelis, K., Pinto, P. C., Henrist, B., Métral, E., Neupert, H., Rumolo, G., Shaposhnikova, E., and Taborelli, M. "Amorphous carbon coatings for the mitigation of electron cloud in the CERN Super Proton Synchrotron," Physical Review Special Topics - Accelerators and Beams Vol. 14, No. 7, 2011, p. 071001.

[34] Larciprete, R., Grosso, D. R., Commisso, M., Flammini, R., and Cimino, R. "Secondary electron yield of $\mathrm{Cu}$ technical surfaces: Dependence on electron irradiation," Physical Review Special Topics - Accelerators and Beams Vol. 16, No. 1, 2013, p. 011002.

[35] Larciprete, R., Grosso, D. R., Di Trolio, A., and Cimino, R. "Evolution of the secondary electron emission during the graphitization of thin C films," Applied Surface Science Vol. 328, No. 0, 2015, pp. 356-360.

doi: http://dx.doi.org/10.1016/j.apsusc.2014.12.046

[36] Garwin, E. L., King, F. K., Kirby, R. E., and Aita, O. "Surface properties of metal-nitride and metal-carbide films deposited on $\mathrm{Nb}$ for radio-frequency superconductivity," Journal of Applied Physics Vol. 61, No. 3, 1987, pp. 1145-1154. doi: doi:http://dx.doi.org/10.1063/1.338159

[37] Noer, R., Mitsunobu, S., Kijima, Y., Higuchi, T., and Saito, K. "Secondary electron yield of Nb rf cavity surfaces," The Tenth Workshop on RF Superconductivity Tsukuba, Japan, 2001, pp. 400-402. 
[38] Henrist, B., Hilleret, N., Scheuerlein, C., and Taborelli, M. "The secondary electron yield of TiZr and TiZrV non-evaporable getter thin film coatings," Applied Surface Science Vol. 172, No. 1-2, 2001, pp. 95-102. doi: http://dx.doi.org/10.1016/S0169-4332(00)00838-2

[39] Benvenuti, C., Cazeneuve, J. M., Chiggiato, P., Cicoira, F., Escudeiro Santana, A., Johanek, V., Ruzinov, V., and Fraxedas, J. "A novel route to extreme vacua: The nonevaporable getter thin film coatings," Vacuum Vol. 53, No. 1-2, 1999, pp. 219-225. doi: http://dx.doi.org/10.1016/S0042-207X(98)00377-7

[40] Harkay, K. C., and Rosenberg, R. A. "Properties of the electron cloud in a high-energy positron and electron storage ring," Physical Review Special Topics - Accelerators and Beams Vol. 6, No. 3, 2003, p. 034402.

[41] Wang, J. Q., Guo, Z. Y., Liu, Y. D., Qin, Q., Xing, J., and Zhao, Z. "Electron cloud instability studies in the Beijing Electron Positron Collider," Physical Review Special Topics - Accelerators and Beams Vol. 7, No. 9, 2004, p. 094401.

[42] Grosso, D. R., Commisso, M., Cimino, R., Larciprete, R., Flammini, R., and Wanzenberg, R. "Effect of the surface processing on the secondary electron yield of Al alloy samples," Physical Review Special Topics - Accelerators and Beams Vol. 16, No. 5, 2013, p. 051003.

[43] Upadhyay, J., Im, D., Popović, S., Valente-Feliciano, A. M., Phillips, L., and Vušković, L. "Plasma processing of large curved surfaces for superconducting rf cavity modification," Physical Review Special Topics - Accelerators and Beams Vol. 17, No. 12, 2014, p. 122001.

[44] Doleans, M., Tyagi, P. V., Afanador, R., McMahan, C. J., Ball, J. A., Barnhart, D. L., Blokland, W., Crofford, M. T., Degraff, B. D., Gold, S. W., Hannah, B. S., Howell, M. P., Kim, S. H., Lee, S. W., Mammosser, J., Neustadt, T. S., Saunders, J. W., Stewart, S., Strong, W. H., Vandygriff, D. J., and Vandygriff, D. M. "In-situ plasma processing to increase the accelerating gradients of superconducting radio-frequency cavities," Nuclear Instruments and Methods in Physics Research Section A: Accelerators, Spectrometers, Detectors and Associated Equipment Vol. 812, 2016, pp. 50-59.

doi: http://dx.doi.org/10.1016/j.nima.2015.12.043

[45] Grundner, M., and Halbritter, J. "XPS and AES studies on oxide growth and oxide coatings on niobium," Journal of Applied Physics Vol. 51, No. 1, 1980, pp. 397-405. doi: doi:http://dx.doi.org/10.1063/1.327386

[46] Lilje, L., Antoine, C., Benvenuti, C., Bloess, D., Charrier, J. P., Chiaveri, E., Ferreira, L., Losito, R., Matheisen, A., Preis, H., Proch, D., Reschke, D., Safa, H., Schmüser, P., Trines, D., Visentin, B., and Wenninger, H. "Improved surface treatment of the superconducting TESLA cavities," Nuclear Instruments and Methods in Physics Research Section A: Accelerators, Spectrometers, Detectors and Associated Equipment Vol. 516, No. 2-3, 2004, pp. 213-227.

doi: http://dx.doi.org/10.1016/j.nima.2003.08.116

[47] Geng, R. L., Knobloch, J., and Padamsee, H. "Miscro-structures of RF surfaces in the electron-beam weld regions of niobium," Proceedengs of the 1999 Workshop on RF Superconductivity. Santa Fe, New Mexico, USA, 1999, pp. 238-245.

[48] Diepers, H., Schmidt, O., Martens, H., and Sun, F. S. "A new method of electropolishing niobium," Physics Letters A Vol. 37, No. 2, 1971, pp. 139-140.

doi: http://dx.doi.org/10.1016/0375-9601(71)90095-8 
[49] Kneisel, P. "Surface preparation of niobium," Proceedings of the Sixth Workshop on RF Superconductivity. Karlsruhe, Germany, 1980, pp. 27-40.

[50] Upadhyay, J., Im, D., Popović, S., Valente-Feliciano, A.-M., Phillips, L., and Vušković, L. "Etching mechanism of niobium in coaxial $\mathrm{Ar} / \mathrm{Cl}_{2}$ radio frequency plasma," Journal of Applied Physics Vol. 117, No. 11, 2015, p. 113301. doi: doi:http://dx.doi.org/10.1063/1.4914298

[51] You, J. B., Zhang, X. W., Cai, P. F., Dong, J. J., Gao, Y., Yin, Z. G., Chen, N. F., Wang, R. Z., and Yan, H. "Enhancement of field emission of the $\mathrm{ZnO}$ film by the reduced work function and the increased conductivity via hydrogen plasma treatment," Applied Physics Letters Vol. 94, No. 26, 2009, p. 262105. doi: doi:http://dx.doi.org/10.1063/1.3167301

[52] Ballantini, R., Dacca, A., Gemme, G., and Parodi, R. "Improvement of the maxiumum field of accelerating cavities by dry oxidation," Proceedings of the Ninth Workshop on RF Superconductivity. Santa Fe, New Mexico, 1999, pp. 211-217.

[53] Tyagi, P. V., Doleans, M., Hannah, B., Afanador, R., McMahan, C., Stewart, S., Mammosser, J., Howell, M., Saunders, J., Degraff, B., and Kim, S. H. "Improving the work function of the niobium surface of SRF cavities by plasma processing," Applied Surface Science Vol. 369, 2016, pp. 29-35. doi: http://dx.doi.org/10.1016/j.apsusc.2016.02.030

[54] Basovic, M., Samolov, A., Cuckov, F., Popović, S., Tomovic, M., and Vušković, L. "Effects of plasma processing on secondary electron yield of niobium samples," Proceedings of IPAC. Richmond, Virginia, USA, 2015, pp. 3558-3560.

[55] Montgomery, D. C., and Runger, C. R. "A prediction interval for a future observation," Applied Statistics and Probability for Engineers. Third edition ed., John Wiley \& Sons, Inc., 2002, pp. 268-270.

[56] Farnsworth, H. E. "Electronic bombardment of metal surfaces," Physical Review Vol. 25, No. 1, 1925, pp. 41-57.

[57] Cimino, R. "Surface related properties as an essential ingredient to e-cloud simulations," Nuclear Instruments and Methods in Physics Research Section A: Accelerators, Spectrometers, Detectors and Associated Equipment Vol. 561, No. 2, 2006, pp. 272-275. doi: http://dx.doi.org/10.1016/j.nima.2006.01.042 


\section{VITA}

Miloš Bašović

Department of Mechanical and Aerospace Engineering

Old Dominion University

Norfolk, VA 23529

Education

May 2016 Doctor of Philosophy in Mechanical Engineering, Old Dominion University

July 2010 Master of Science in Mechanical Engineering, University of Belgrade, Serbia

Sept 2008 Bachelor of Science in Mechanical Engineering, University of Belgrade, Serbia

Publications

- Basovic, M., Jovanovic, V., Cuckov, F., and Tomovic, M. "Design of a mechatronic system for accurate measurement of secondary electron emission," (submitted to Measurement).

- Upadhyay, J., Im, D., Peshl, J., Bašović, M., Popović, S., Valente-Feliciano, A. M., Phillips, L., and Vušković, L. "Apparatus and method for plasma processing of SRF cavities," Nuclear Instruments and Methods in Physics Research Section A: Accelerators, Spectrometers, Detectors and Associated Equipment Vol. 818, 2016, pp. 76-81.

- Basovic, M., Samolov, A., Cuckov, F., Popović, S., M, T., and Vušković, L. "Secondary electron yield of electron beam welded areas of SRF cavities," Proceedings of the Seventeenth Workshop on RF Superconductivity Whistler, Canada, 2015.

- Basovic, M., Samolov, A., Cuckov, F., Popović, S., Tomovic, M., and Vušković, L. "Effects of plasma processing on secondary electron yield of niobium samples," Proceedings of IPAC. Richmond, Virginia, USA, 2015, pp. 3558-3560.

Awards and Honors

- Student Travel Assistance $-68^{\text {th }}$ Gaseous Electronics Conference, Oct 2015.

- Graduate Research Assistanship - Thomas Jefferson National Accelerator Facility, Jan 2011 - Aug 2015.

- Student Grant - 6th International Particle Accelerator Conference, May 2015.

- The Honor Society of Phi Kappa Phi Chapter 200 Scholarship, Apr 2015.

- Frank Batten College of Engineering and Technology Faculty Award in Mechanical Engineering, Apr 2015.

- Department of Mechanical and Aerospace Engineering Award for Outstanding Achievment, Apr 2015.

- Student Travel Assistance - 67th Gaseous Electronics Conference, Nov 2014

\section{Professional Affiliations}

- American Society of Mechanical Engineers, $A S M E$

- Society of Manufacturing Engineers, $S M E$

- American Physical Society, APS 